\title{
Revisiting the vascular theory of glaucoma using optical coherence tomography angiography
}

Citation for published version (APA):

Rao, H. (2018). Revisiting the vascular theory of glaucoma using optical coherence tomography angiography. [Doctoral Thesis, Maastricht University]. Maastricht University. https://doi.org/10.26481/dis.20180209hblr

Document status and date:

Published: 01/01/2018

DOI:

10.26481/dis.20180209hblr

Document Version:

Publisher's PDF, also known as Version of record

\section{Please check the document version of this publication:}

- A submitted manuscript is the version of the article upon submission and before peer-review. There can be important differences between the submitted version and the official published version of record. People interested in the research are advised to contact the author for the final version of the publication, or visit the DOI to the publisher's website.

- The final author version and the galley proof are versions of the publication after peer review.

- The final published version features the final layout of the paper including the volume, issue and page numbers.

Link to publication

\footnotetext{
General rights rights.

- You may freely distribute the URL identifying the publication in the public portal. please follow below link for the End User Agreement:

www.umlib.nl/taverne-license

Take down policy

If you believe that this document breaches copyright please contact us at:

repository@maastrichtuniversity.nl

providing details and we will investigate your claim.
}

Copyright and moral rights for the publications made accessible in the public portal are retained by the authors and/or other copyright owners and it is a condition of accessing publications that users recognise and abide by the legal requirements associated with these

- Users may download and print one copy of any publication from the public portal for the purpose of private study or research.

- You may not further distribute the material or use it for any profit-making activity or commercial gain

If the publication is distributed under the terms of Article $25 \mathrm{fa}$ of the Dutch Copyright Act, indicated by the "Taverne" license above, 


\title{
REVISITING THE VASCULAR THEORY OF GLAUCOMA USING OPTICAL COHERENCE TOMOGRAPHY ANGIOGRAPHY
}

\author{
DISSERTATION \\ to obtain the degree of Doctor at Maastricht University, \\ on the authority of the Rector Magnificus, \\ Prof. Dr. Rianne M. Letschert \\ in accordance with the decision of the Board of Deans, \\ to be defended in public on \\ Friday February 9th, 2018 at 10.00 hours \\ by \\ HARSHA RAO
}




\section{Supervisors:}

Prof. Dr. C.A.B. Webers,

Dr. R.N. Weinreb, University of California San Diego

\section{Assessment Committee:}

Prof. Dr. J.E. Wildberger, (chairman)

Prof. Dr. H.J.G.M. Crijns

Prof. Dr. J.F.C. Glatz

Prof. Dr. N.M. Jansonius (UMC Groningen)

Dr. T. Theelen, (Radboud UMC Nijmegen) 
To all my mentors, my entire family and friends 



\section{TABLE OF CONTENTS}

1. Introduction

2. Repeatability of vessel density measurements of optical coherence tomography angiography in normal and glaucoma eyes

Br J Ophthalmol (Accepted for publication)

3. Determinants of peripapillary and macular vessel densities measured by optical coherence tomography angiography in normal eyes

J Glaucoma 2017 May;26(5):491-497

4. Regional comparisons of optical coherence tomography angiography vessel density in primary open angle glaucoma Am J Ophthalmol 2016 Nov;171:75-83

5. A comparison of the diagnostic ability of vessel density and structural measurements of optical coherence tomography in primary open angle glaucoma PLoS One 2017 Mar 13;12(3):e0173930

6. Diagnostic ability of peripapillary vessel density measurements of optical coherence tomography angiography in primary open-angle and angle-closure glaucoma

Br J Ophthalmol 2017 Aug;101(8):1066-1070

7. Vessel density and structural measurements of optical coherence tomography in primary angle closure and primary angle closure glaucoma Am J Ophthalmol 2017 May;177:106-115 
8. Relationship of optic nerve structure and function to peripapillary vessel density measurements of optical coherence tomography angiography in glaucoma J Glaucoma 2017 Jun;26(6):548-554

9. Optical coherence tomography angiography vessel density measurements in eyes with primary open angle glaucoma and disc hemorrhage J Glaucoma 2017 Oct;26(10):888-895

10. Discussion 165

11. Valorization addendum

12. Summary 179

13. Curriculum vitae 185 


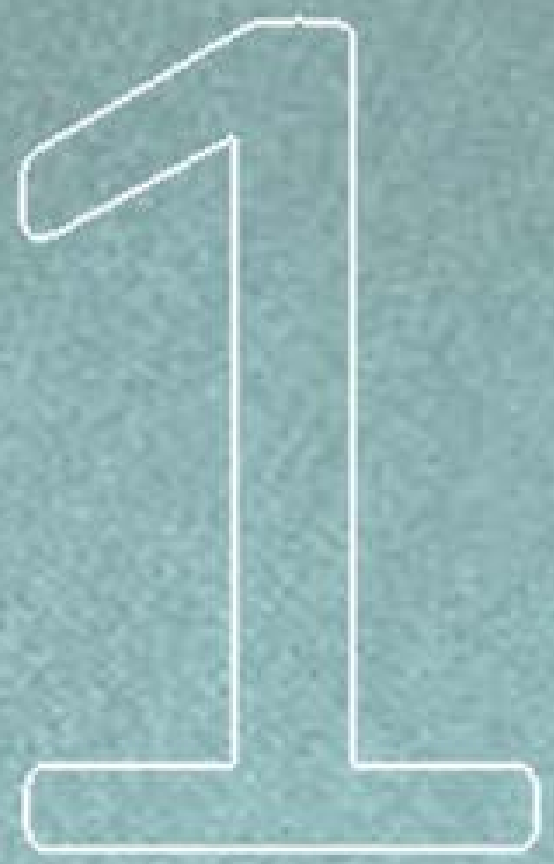

Introduction 


\section{INTRODUCTION}

Glaucoma is a chronic progressive optic neuropathy resulting from the apoptosis of retinal ganglion cells (RGC). ${ }^{1}$ It is characterized by typical optic disc and retinal nerve fiber layer (RNFL) changes with or without visual field (VF) changes. The standard tests used to assess the amount of structural (optic disc and RNFL) changes in current day clinical practice are optic disc photography (Figure 1, upper panel) and optical coherence tomography (OCT, Figure 1, middle panel). OCT also images the inner retinal layers at the macular region (nerve fiber layer, ganglion cell layer and inner plexiform layer), together called the ganglion cell complex (GCC), which is also shown to be affected in glaucoma. ${ }^{2-4}$ The standard test used to assess the amount of functional (VF) loss is the standard automated perimetry (SAP, Figure 1, lower panel). Intraocular pressure is the most important risk factor for the development and progression of glaucoma. ${ }^{5-8}$

Glaucoma is the leading cause of irreversible blindness in the world. A recent systematic review and meta-analysis estimated that the number of people aged between 40 and 80 years with glaucoma worldwide is 64.3 million, and that it will increase to 76.0 million in 2020 and 111.8 million in $2040 .{ }^{9}$ Another recent meta-analysis reported that the number of people blind and visually impaired due to glaucoma worldwide is 2.1 million and 4.2 million respectively. ${ }^{10}$ The meta-analysis also reported that between 1990 and 2010, the number of people blind and the number of people visually impaired due to glaucoma increased by 0.8 million or $62 \%$ and by 2.3 million or $83 \%$, respectively. ${ }^{10}$

\section{Pathogenesis of Glaucoma}

Although the exact pathogenesis of glaucoma is not fully understood, two theories have been proposed to explain the RGC apoptosis in glaucoma. ${ }^{11}$ The "mechanical theory" proposes RGC death to be a direct consequence of intraocular pressure (IOP). It proposes that IOP causes a mechanical obstruction to the axoplasmic flow in the axons of the RGC at the lamina cribrosa leading to RGC death. ${ }^{12}$ 

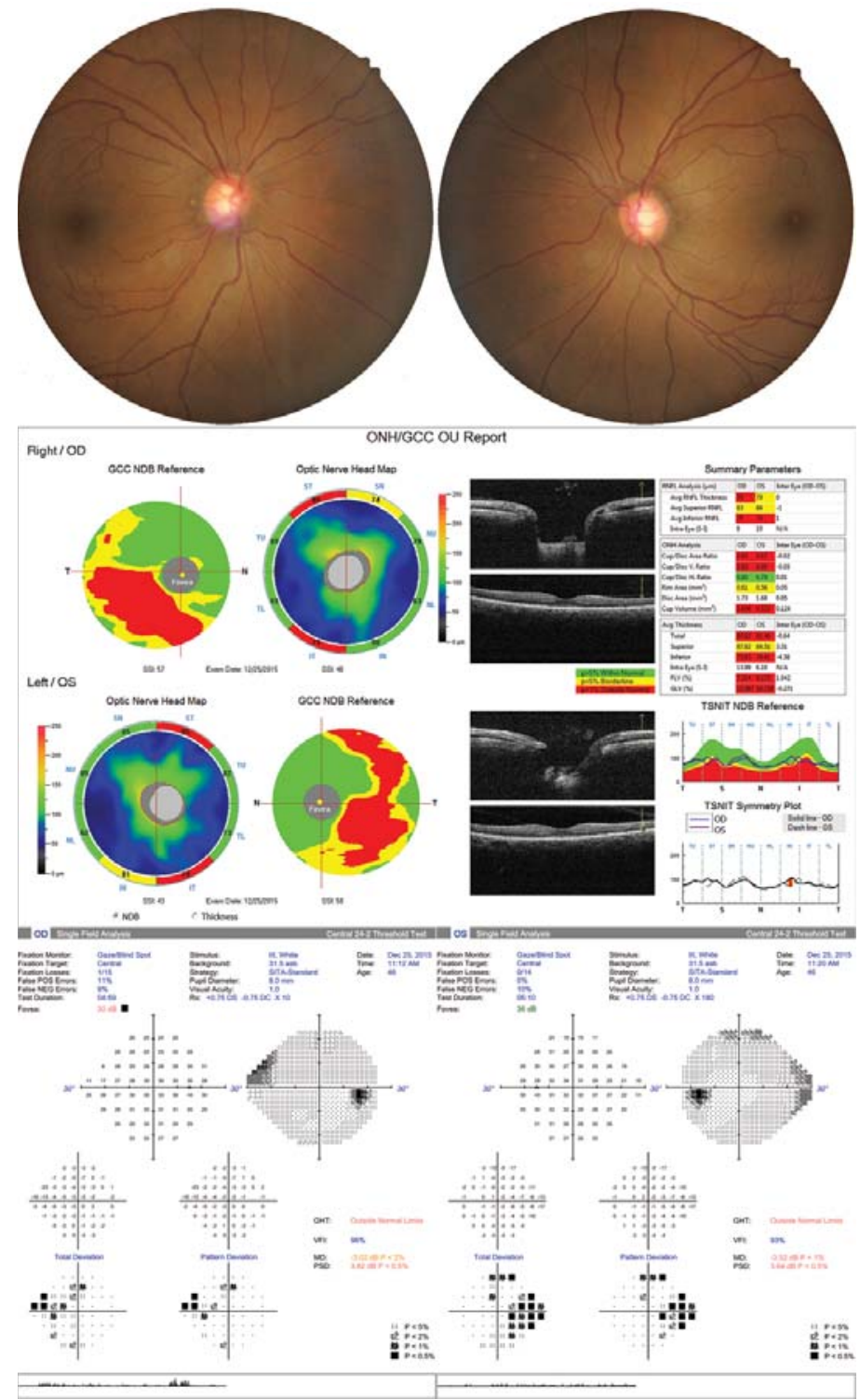

Figure 1. Top panel shows the optic disc photographs of the right and left eye of a patient with glaucoma with neuroretinal rim thinning and retinal nerve fiber layer (RNFL) defects in superotemporal and inferotemporal regions of both eyes. Middle panel shows optical coherence tomography (OCT) image with RNFL thinning correlating with the findings on the disc photographs. OCT image also shows the ganglion cell complex thinning in both eyes. Lower panel shows the standard automated perimetry printouts showing nasal defects in both right and left eyes. 
Multiple studies have reported IOP to be a major causal factor for glaucoma, with the risk of incident glaucoma and its progression increasing with higher IOP. ${ }^{5-8 \text {, }}$ 13-16 However, it is well accepted that the mechanical theory alone fails to explain the entire pathogenic mechanism of glaucoma because glaucoma occurs and progresses even at normal IOP levels in a significant number of eyes and not all eyes with high IOP develop glaucoma. The "vascular theory", the second theory to explain the pathogenic mechanism of glaucoma, proposes reduced blood supply to the RGCs, either due to increased IOP or due to other (vascular) risk factors, as the cause of glaucoma. ${ }^{17-19}$

\section{Ocular blood supply: Anatomy and physiology}

Ocular blood supply occurs predominantly through the retinal and the choroidal circulations. The anatomy and physiology of ocular blood flow has been enumerated in previous studies. ${ }^{20,21}$ In brief, retinal circulation is through central retinal artery, which is a branch of the ophthalmic artery. Retinal circulation is a low flow, high oxygen extraction system with no autonomic innervation. The presence of endothelial tight junctions results in a blood-retinal barrier, similar to the blood - brain barrier. Retinal circulation has autoregulation so that the blood flow is held fairly constant in spite of mild to moderate changes in the perfusion pressure and IOP. In contrast, choroidal circulation is a high flow, low oxygen extraction system. The choroid is supplied by the posterior ciliary arteries, which are branches of the ophthalmic artery. Choroid has a rich autonomic innervation and the endothelium of the choroidal vessels are fenestrated. The choroidal circulation has poor autoregulation, which renders the choroidal blood flow more dependent on perfusion pressure. The ONH blood supply also has been studied in great detail. 22,23 The superficial layer of the $\mathrm{ONH}$ receives its blood supply via small branches of the central retinal artery. The prelaminar region is supplied by branches from recurrent choroid arterioles and the short posterior ciliary arteries. 


\section{Measuring ocular blood flow in humans}

Retinal and ONH blood flow in glaucoma eyes has been investigated earlier using various techniques. Fluorescein angiography (FA), a common technique used to evaluate vasculature in various retinal pathologies, has been used to investigate ocular blood flow in glaucoma. And the studies with FA have reported prolonged arteriovenous passage times, ${ }^{24,} 25$ fluorescein filling defects in the disc, ${ }^{26,} 27$ focal sector hypoperfusion of the optic disc and diffuse disc hypo-perfusion ${ }^{28}$ in patients with glaucoma. However, FA is an invasive technique requiring the intravenous injection of a dye and has difficulty in quantification.

Laser Doppler flowmetry (LDF) and laser speckleflowgraphy (LSFG) are two other non-invasive techniques that have been used to measure ONH perfusion. Multiple studies with these two techniques have reported significantly reduced neuroretinal rim blood flow and peripapillary retinal blood flow in patients with glaucoma compared to controls. ${ }^{29-33}$ However, measurements provided by LDF and LSFG are too variable for diagnostic application. Coefficient of variation (CV) for intra-visit repeatability with LDF has been reported to range from $6.6 \%$ to $21.2 \%$ and for inter-visit reproducibility from $25.2 \%$ to $30.1 \%$. $34-38$ With LSFG, CVs for intra-visit repeatability has been reported to range from $1.9 \%$ to $11.9 \%$, and inter-visit reproducibility was $12.8 \%{ }^{34,39-41}$

\section{OCT angiography}

A number of algorithms and/or techniques using OCT have been developed for imaging the vasculature of the eye. Doppler OCT was one of the earliest technique developed for vascular imaging. It assessed blood flow by comparing phase differences between adjacent A-scans. ${ }^{42}$ Although Doppler OCT was appropriate for large vessels around the disc, it was not sensitive enough to measure accurately the low velocities in small vessels that make up the $\mathrm{ONH}$ and retinal microcirculation. 
Of the several OCT-based techniques that have been developed to image microvascular networks in human eyes in vivo, optical microangiography (OMAG) was a technique that was able to resolve the fine vasculature in both retinal and choroid layers. ${ }^{43}$ OMAG worked by using a modified Hilbert transform to separate the scattering signals from static and moving scatters. ${ }^{44}$ However, the high sensitivity of OMAG required precise removal of bulk-motion by resolving the Doppler phase shift. ${ }^{45}$ Thus it was susceptible to artifacts from system or biological phase instability.

Recently, OCT has been used to develop a non-invasive three-dimensional angiography algorithm called split spectrum amplitude-decorrelation angiography (SSADA) for imaging the ONH microcirculation. ${ }^{46}$ SSADA uses motion of the blood column as a contrast to delineate blood vessels from static tissue. The principles of SSADA has been explained in detail by Jia et al. ${ }^{46}$ The high axial resolution of OCT makes it sensitive to the pulsatile bulk motion noise in the axial direction. SSADA splits the raw full spectrum of OCT signal into multiple spectrums, each with a narrow bandwidth to intentionally lower the axial resolution. This minimizes the pulsatory bulk motion noise along the axial direction and optimizes flow detection along the transverse direction. After the narrower spectra are Fourier-transformed, low resolution OCT amplitude frames are used to calculate decorrelation. Inter-B-scan decorrelation is determined at each of the narrower spectral bands separately and then averaged. Recombining the decorrelation images from the multiple narrow spectral bands yields high quality cross-sectional angiograms that uses the full information in the entire OCT spectral range. ${ }^{46}$

OCT angiography (OCTA) is performed using a set of 2 scans; one vertical priority $(X)$ and one horizontal priority $(Y)$ raster volumetric scan. In the prototype setup, which used a swept-source OCT platform, each B-scan both in the vertical and horizontal direction was repeated 8 times at every position. ${ }^{47}$ The blood flow resulted in fluctuation in the amplitude of OCT fringes as RBCs entered and exited a particular voxel (which is portmanteau of "volume" and "pixel"). The fluctuating values of OCT intensities was considered as the decorrelation (D). Hence the eight B-scan frames contained fluctuating values of OCT output intensities at any given voxel in the flow of blood, and the definition of $D$ was constructed so that 
fluctuating intensities yielded high D values (approaching 1). Pixels in the B-scan frames that contained static tissue and hence constant intensities yielded small D values (approaching 0$) .47$

In summary, therefore, D was a function of the flow velocity regardless of direction, given the RBCs concentration was constant. The faster blood particles moved across the laser beam, the higher $D$ of the received signals within a velocity range set by the scan parameters. In the other words, D was proportional to flow velocity, but it could be saturated at the maximum detectable flow velocity, due to a limit of time resolution for each scan parameters. ${ }^{46}$

OCTA quantifies the ocular circulation using two parameters: flow index and vessel density. Flow index is defined as the average decorrelation values in the measured area and vessel density is defined as the percentage area occupied by vessels in the measured area. ${ }^{47}$ The threshold decorrelation value used to separate blood vessel and static tissue was set at 0.125 , which was two standard deviations above the mean decorrelation value in the foveal avascular zone, a region devoid of vessels.

Two other technologies that helped OCTA possible were the en face presentation and motion correction. En face presentation helps to reduce the data complexity of the 3 dimensional scans and presents angiography information in 2 dimension. Retina is segmented into different slabs, like choriocapillaris, deep retina, outer retina and superficial retina, and vessels in each of these slabs are presented in 2 dimensional format (Figure 2). ONH is similarly segmented into choroid, nerve head, radial peripapillary capillary and vitreous slabs (Figure 3 ). The angiograms are generated by projecting the maximum decorrelation for each transverse position within the segmented depth range, representing the fastest flowing vessel lumen in the segmented tissue layers. As the time required to obtain the scan with OCTA is close to 3 seconds, involuntary saccades and changes in fixation during data acquisition can lead to motion artifacts that may confound the interpretation of the final OCT angiogram. "Motion Correction Technology"(MCT) is an orthogonal registration algorithm which minimizes these motion artifacts. ${ }^{48}$ 


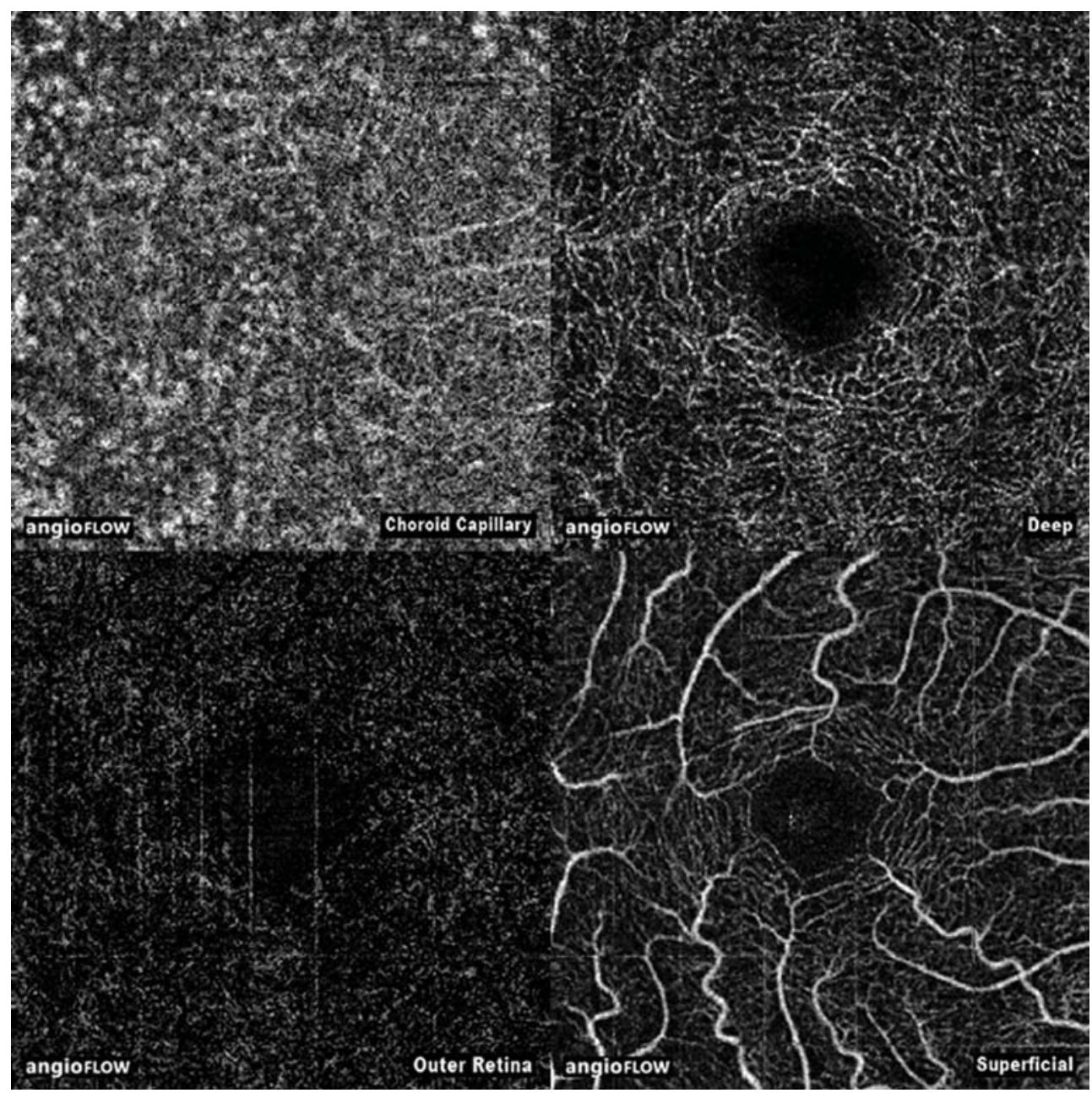

Figure 2. Angiography slabs of the macular scan obtained using spectral domain optical coherence tomography showing the choriocapillaris, deep retinal, outer retinal and superficial retinal layers.

Initial studies with OCTA, from the group that developed the technology, demonstrated reduced flow index and vessel density in eyes with primary open angle glaucoma (POAG) compared to control eyes. 47,49

Subsequently, SSADA was optimized for the spectral-domain OCT (SDOCT) platform. ${ }^{50}$ Two sequential B-scans were performed at each location for flow 


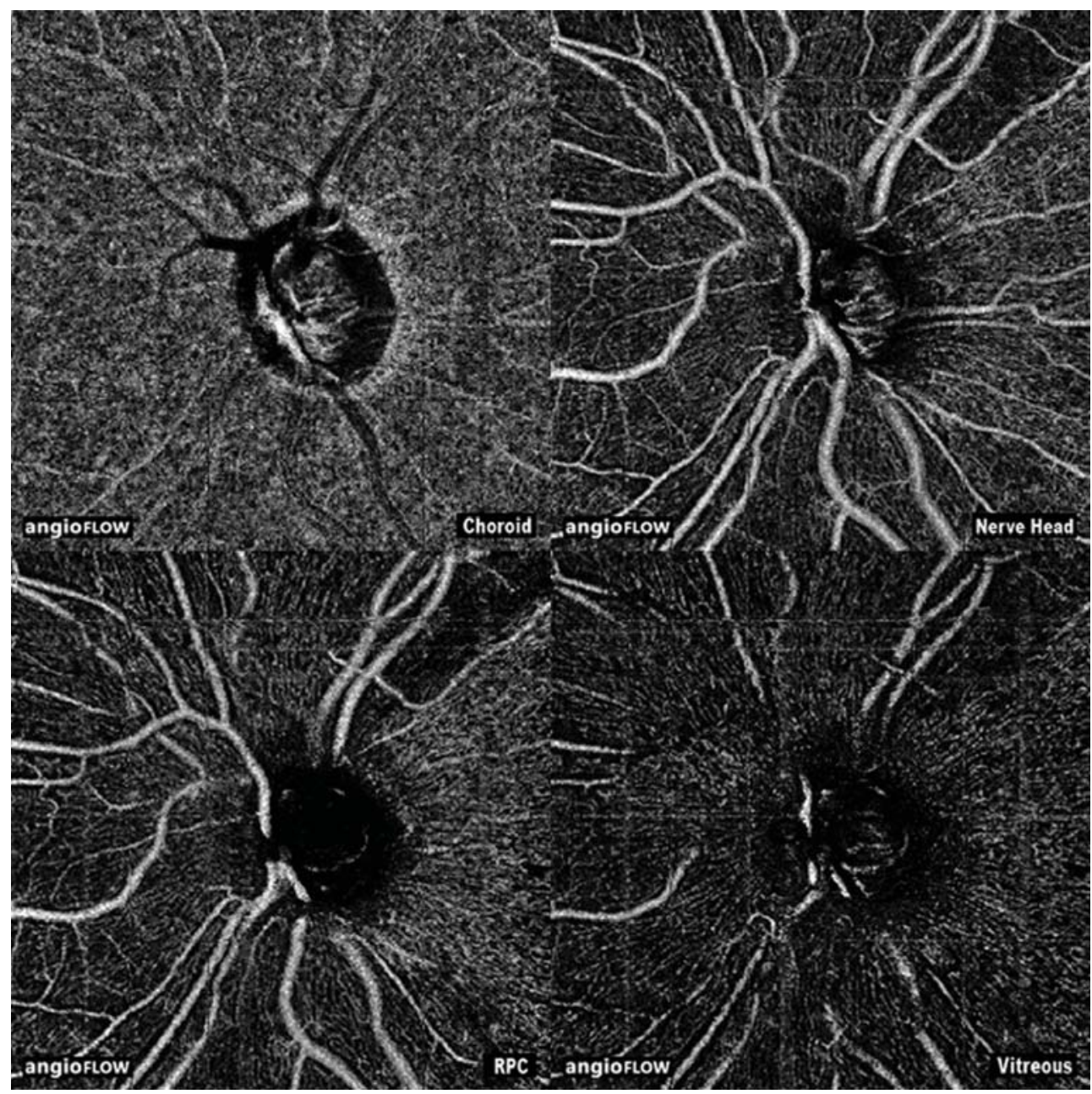

Figure 3. Angiography slabs of the optic nerve head scan obtained using spectral domain optical coherence tomography showing the choroid, nerve head, radial peripapillary capillary and vitreous layers.

detection. The availability of SSADA on the SDOCT platform made the technology commercially available for clinicians. Subsequently, the same group of researchers who developed the technology also evaluated the peripapillary flow index and vessel density on OCTA and found that both these parameters were significantly reduced in patients with glaucoma as compared to control eyes. ${ }^{51}$ All these initial studies however had small sample sizes. 


\section{Aims and outline of the thesis}

The aim of the thesis is to understand the clinical utility of OCTA in glaucoma. If the new parameters measured on OCTA are able to diagnose glaucomatous damage earlier than the standard methods (structural measurements like RNFL and GCC thickness), this could lead to a paradigm shift in the way glaucoma is diagnosed and monitored. This could also possibly change the way in which treatment outcomes are evaluated. The chapters subsequently elaborate the step-by-step approach we employed to explore the clinical utility of OCTA.

In Chapter 2, the intra-session repeatability of vessel density measurements of OCTA is evaluated in normal eyes and eyes with glaucoma separately. Knowing the test-retest variability is important to decide the change in vessel density measurements that can be considered clinically significant.

In Chapter 3, the determinants of OCTA-measured peripapillary and macular vessel densities of normal eyes are evaluated. This chapter highlights the eye-related, subject-related and technology-related factors affecting the measurements of vessel densities in normal eyes.

In Chapter 4, the diagnostic ability of the OCTA-measured vessel densities within the $\mathrm{ONH}$, in the peripapillary and macular regions is evaluated. Knowing the diagnostic ability helps us to determine if subsequent evaluation of this new technology is beneficial in glaucoma. Also, the effect of the covariates, such as disease severity and baseline IOP (pre-treatment IOP) on the diagnostic abilities of vessel densities are evaluated.

In Chapter 5, the diagnostic abilities of the OCTA-measured vessel densities within the $\mathrm{ONH}$, in the peripapillary and macular regions are compared with that of the OCT-measured structural measurements in these regions, namely, neuroretinal rim area, RNFL thickness and macular GCC thickness measurements.

In Chapter 6, the diagnostic abilities of OCTA-measured peripapillary vessel density is compared in eyes with POAG and PACG. Also, the diagnostic abilities 
of peripapillary vessel densities are compared with RNFL thickness separately in POAG and PACG.

In Chapter 7, a more detailed evaluation of the diagnostic abilities of OCTAmeasured vessel densities within the $\mathrm{ONH}$ and in the peripapillary and macular regions in eyes with PACG is conducted. These diagnostic abilities are compared against those of the rim area, RNFL thickness and GCC thickness measurements. Also, the vessel densities in eyes with primary angle closure (eyes with a history of high IOP but with normal optic disc and visual fields) are compared with control eyes to evaluate the effect of high IOP on the vessel densities.

In Chapter 8, the structure-structure association between OCTA-measured peripapillary vessel density and RNFL thickness, and structure-function association between peripapillary vessel density and visual sensitivity loss on perimetry in POAG eyes are determined.

In Chapter 9, the measurements of OCTA-derived vessel densities in POAG eyes with disc hemorrhage are compared with that of severity-matched POAG eyes without disc hemorrhage. This indirectly evaluates if there is a vascular abnormality in POAG eyes that show disc hemorrhages.

\section{REFERENCES}

1. Weinreb RN, Khaw PT. Primary open-angle glaucoma. Lancet 2004;363:1711-20.

2. Rao HL, Zangwill LM, Weinreb RN, et al. Comparison of different spectral domain optical coherence tomography scanning areas for glaucoma diagnosis. Ophthalmology 2010;117:1692-9.

3. Rao HL, Babu JG, Addepalli UK, et al. Retinal nerve fiber layer and macular inner retina measurements by spectral domain optical coherence tomograph in Indian eyes with early glaucoma. Eye (Lond) 2012;26:133-9.

4. Rao HL, Addepalli UK, Chaudhary S, et al. Ability of different scanning protocols of spectral domain optical coherence tomography to diagnose preperimetric glaucoma. Invest Ophthalmol Vis Sci 2013;54:7252-7.

5. Sommer A, Tielsch JM, Katz J, et al. Relationship between intraocular pressure and primary open angle glaucoma among white and black Americans. The Baltimore Eye Survey. Arch Ophthalmol 1991;109:1090-5. 


\section{Chapter 1}

6. The Advanced Glaucoma Intervention Study (AGIS): 7. The relationship between control of intraocular pressure and visual field deterioration. The AGIS Investigators. Am J Ophthalmol 2000;130:429-40.

7. Kass MA, Heuer DK, Higginbotham EJ, et al. The Ocular Hypertension Treatment Study: a randomized trial determines that topical ocular hypotensive medication delays or prevents the onset of primary open-angle glaucoma. Arch Ophthalmol 2002;120:701-13.

8. Leske MC, Heijl A, Hussein M, et al. Factors for glaucoma progression and the effect of treatment: the Early Manifest Glaucoma Trial. Arch Ophthalmol 2003;121:48-56.

9. Tham YC, Li X, Wong TY, et al. Global prevalence of glaucoma and projections of glaucoma burden through 2040: a systematic review and meta-analysis. Ophthalmology 2014;121:208190.

10. Bourne RR, Taylor HR, Flaxman SR, et al. Number of People Blind or Visually Impaired by Glaucoma Worldwide and in World Regions 1990 - 2010: A Meta-Analysis. PLoS One 2016;11:e0162229.

11. Fechtner RD, Weinreb RN. Mechanisms of optic nerve damage in primary open angle glaucoma. Surv Ophthalmol 1994;39:23-42.

12. Yan DB, Coloma FM, Metheetrairut A, et al. Deformation of the lamina cribrosa by elevated intraocular pressure. Br J Ophthalmol 1994;78:643-8.

13. Francis BA, Varma R, Chopra V, et al. Intraocular pressure, central corneal thickness, and prevalence of open-angle glaucoma: the Los Angeles Latino Eye Study. Am J Ophthalmol 2008;146:741-6.

14. Lichter PR, Musch DC, Gillespie BW, et al. Interim clinical outcomes in the Collaborative Initial Glaucoma Treatment Study comparing initial treatment randomized to medications or surgery. Ophthalmology 2001;108:1943-53.

15. Weinreb RN, Aung T, Medeiros FA. The pathophysiology and treatment of glaucoma: a review. JAMA 2014;311:1901-11.

16. Ernest PJ, Schouten JS, Beckers HJ, et al. An evidence-based review of prognostic factors for glaucomatous visual field progression. Ophthalmology 2013;120:512-9.

17. Flammer J. The vascular concept of glaucoma. Surv Ophthalmol 1994;38 Suppl:S3-6.

18. Bonomi L, Marchini G, Marraffa M, et al. Vascular risk factors for primary open angle glaucoma: the Egna-Neumarkt Study. Ophthalmology 2000;107:1287-93.

19. Leske MC, Heijl A, Hyman L, et al. Predictors of long-term progression in the Early Manifest Glaucoma Trial. Ophthalmology 2007;114:1965-72.

20. Bill A, Nilsson SF. Control of ocular blood flow. J Cardiovasc Pharmacol 1985;7 Suppl 3:S96102.

21. Flammer J, Orgul S. Optic nerve blood-flow abnormalities in glaucoma. Prog Retin Eye Res 1998;17:267-89.

22. Hayreh SS. Blood supply of the optic nerve head. Ophthalmologica 1996;210:285-95.

23. Hayreh SS. The blood supply of the optic nerve head and the evaluation of it - myth and reality. Prog Retin Eye Res 2001;20:563-93. 
24. Arend O, Plange N, Sponsel WE, Remky A. Pathogenetic aspects of the glaucomatous optic neuropathy: fluorescein angiographic findings in patients with primary open angle glaucoma. Brain Res Bull 2004;62:517-24.

25. Huber K, Plange N, Remky A, Arend O. Comparison of colour Doppler imaging and retinal scanning laser fluorescein angiography in healthy volunteers and normal pressure glaucoma patients. Acta Ophthalmol Scand 2004;82:426-31.

26. Talusan E, Schwartz B. Specificity of fluorescein angiographic defects of the optic disc in glaucoma. Arch Ophthalmol 1977;95:2166-75.

27. Schwartz B, Rieser JC, Fishbein SL. Fluorescein angiographic defects of the optic disc in glaucoma. Arch Ophthalmol 1977;95:1961-74.

28. Hitchings RA, Spaeth GL. Fluorescein angiography in chronic simple and low-tension glaucoma. Br J Ophthalmol 1977;61:126-32.

29. Piltz-seymour JR, Grunwald JE, Hariprasad SM, Dupont J. Optic nerve blood flow is diminished in eyes of primary open-angle glaucoma suspects. Am J Ophthalmol 2001;132:63-9.

30. Hamard P, Hamard H, Dufaux J, Quesnot S. Optic nerve head blood flow using a laser Doppler velocimeter and haemorheology in primary open angle glaucoma and normal pressure glaucoma. Br J Ophthalmol 1994;78:449-53.

31. Michelson G, Langhans MJ, Groh MJ. Perfusion of the juxtapapillary retina and the neuroretinal rim area in primary open angle glaucoma. J Glaucoma 1996;5:91-8.

32. Hafez AS, Bizzarro RL, Lesk MR. Evaluation of optic nerve head and peripapillary retinal blood flow in glaucoma patients, ocular hypertensives, and normal subjects. Am J Ophthalmol 2003;136:1022-31.

33. Yokoyama Y, Aizawa N, Chiba N, et al. Significant correlations between optic nerve head microcirculation and visual field defects and nerve fiber layer loss in glaucoma patients with myopic glaucomatous disk. Clin Ophthalmol 2011;5:1721-7.

34. Yaoeda K, Shirakashi M, Funaki S, et al. Measurement of microcirculation in the optic nerve head by laser speckle flowgraphy and scanning laser Doppler flowmetry. Am J Ophthalmol 2000;129:734-9.

35. Kagemann L, Harris A, Chung HS, et al. Heidelberg retinal flowmetry: factors affecting blood flow measurement. Br J Ophthalmol 1998;82:131-6.

36. Luksch A, Lasta M, Polak K, et al. Twelve-hour reproducibility of retinal and optic nerve blood flow parameters in healthy individuals. Acta Ophthalmol 2009;87:875-80.

37. Nicolela MT, Hnik P, Schulzer M, Drance SM. Reproducibility of retinal and optic nerve head blood flow measurements with scanning laser Doppler flowmetry. J Glaucoma 1997;6:15764.

38. Iester $\mathrm{M}$, Altieri $\mathrm{M}$, Michelson $\mathrm{G}$, et al. Intraobserver reproducibility of a two-dimensional mapping of the optic nerve head perfusion. J Glaucoma 2002;11:488-92.

39. Aizawa N, Yokoyama Y, Chiba N, et al. Reproducibility of retinal circulation measurements obtained using laser speckle flowgraphy-NAVI in patients with glaucoma. Clin Ophthalmol 2011;5:1171-6. 
40. Yaoeda K, Shirakashi M, Funaki S, et al. Measurement of microcirculation in optic nerve head by laser speckle flowgraphy in normal volunteers. Am J Ophthalmol 2000;130:606-10.

41. Tamaki Y, Araie M, Tomita K, et al. Real-time measurement of human optic nerve head and choroid circulation, using the laser speckle phenomenon. Jpn J Ophthalmol 1997;41:49-54.

42. Wang $Y$, Bower BA, Izatt JA, et al. Retinal blood flow measurement by circumpapillary Fourier domain Doppler optical coherence tomography. J Biomed Opt 2008;13:064003.

43. An L, Wang RK. In vivo volumetric imaging of vascular perfusion within human retina and choroids with optical micro-angiography. Opt Express 2008;16:11438-52.

44. Wang RK, Jacques SL, Ma Z, et al. Three dimensional optical angiography. Opt Express 2007; 15:4083-97.

45. An L, Subhush HM, Wilson DJ, Wang RK. High-resolution wide-field imaging of retinal and choroidal blood perfusion with optical microangiography. J Biomed Opt 2010;15:026011.

46. Jia Y, Tan O, Tokayer J, et al. Split-spectrum amplitude-decorrelation angiography with optical coherence tomography. Opt Express 2012;20:4710-25.

47. Jia Y, Morrison JC, Tokayer J, et al. Quantitative OCT angiography of optic nerve head blood flow. Biomed Opt Express 2012;3:3127-37.

48. Kraus MF, Potsaid B, Mayer MA, et al. Motion correction in optical coherence tomography volumes on a per A-scan basis using orthogonal scan patterns. Biomed Opt Express 2012;3:1182-99.

49. Jia $Y$, Wei E, Wang $X$, et al. Optical coherence tomography angiography of optic disc perfusion in glaucoma. Ophthalmology 2014;121:1322-32.

50. Gao SS, Liu G, Huang D, Jia Y. Optimization of the split-spectrum amplitude-decorrelation angiography algorithm on a spectral optical coherence tomography system. Opt Lett 2015;40:2305-8.

51. Liu L, Jia Y, Takusagawa HL, et al. Optical Coherence Tomography Angiography of the Peripapillary Retina in Glaucoma. JAMA Ophthalmol 2015;133:1045-52. 


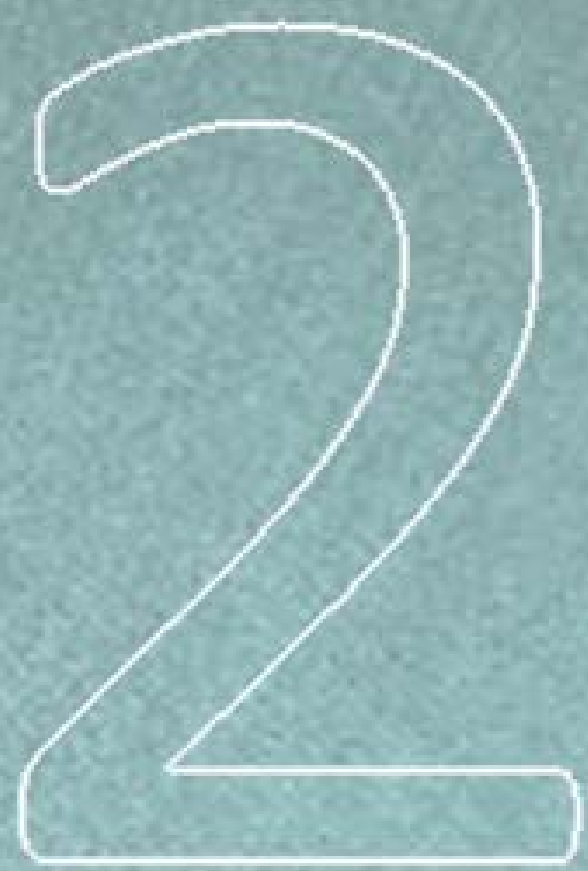

\section{Repeatability of OCT angiography measurements}

Repeatability of vessel density measurements of optical coherence tomography angiography in normal and glaucoma eyes

Jayasree P Venugopal, Harsha L Rao, Robert N Weinreb, Zia S Pradhan, Srilakshmi Dasari, Mohammed Riyazuddin, Narendra K Puttaiah, Dhanaraj A S Rao, Sathi Devi, Kaweh Mansouri, Carroll AB Webers.

BrJ Ophthalmol (Accepted for publication) 


\section{ABSTRACT}

Aims: To compare the intra-session repeatability of peripapillary and macular vessel density measurements of OCT angiography (OCTA) in normal and glaucoma eyes, and to evaluate effect of signal strength of OCTA scans on the repeatability.

Methods: In a cross-sectional study, 3 ONH scans each of 65 eyes ( 30 normal, 35 glaucoma eyes) and 3 macular scans each of 69 eyes ( 35 normal, 34 glaucoma eyes) acquired in the same session with OCTA were analyzed. Repeatability was assessed using within-subject coefficient of repeatability (CRw) and variation $(\mathrm{CVw})$. Effect of signal strength index (SSI) on repeatability was evaluated with repeated measures mixed effects models.

Results: CRw (\%) and CVw (\%) of peripapillary measurements in normal eyes ranged between 3.3 and 7.0, and 2.5 and 4.4 respectively and that in glaucoma eyes between 3.5 and 7.1, and 2.6 and 6.6. For the macular, these measurements ranged between 4.1 and 6.0, and 3.3 and 4.7 in normal eyes and, 4.3 and 6.9, and 3.7 and 5.6 in glaucoma eyes. Repeatability estimates of most measurements were similar in normal and glaucoma eyes. Vessel densities of both peripapillary and macular regions significantly increased with increase in SSI of repeat scans (coefficients ranging from 0.15 to $0.38, \mathrm{p}<0.01$ for all associations).

Conclusions: Repeatability estimates of OCTA measured peripapillary and macular vessel densities were similar in normal eyes and eyes with glaucoma. SSI values of the scans had a significant effect on the repeatability of OCTA with the vessel density values increasing in scans with higher SSI values. 


\section{INTRODUCTION}

A relatively recent application of optical coherence tomography (OCT) has been the development of a three-dimensional angiography algorithm called split spectrum amplitude-decorrelation angiography (SSADA) for imaging the retinal and optic nerve head (ONH) microcirculation non-invasively. ${ }^{1}$ Multiple studies have used OCT angiography (OCTA) to report the vascular changes in common retinal pathologies such as diabetic retinopathy, ${ }^{2}$ age related macular degeneration ${ }^{3,4}$ and retinal vein occlusions. ${ }^{5}$ OCTA has also been used to demonstrate reduced $\mathrm{ONH}$, peripapillary and macular vessel densities in patients with glaucoma. ${ }^{6-11}$

Although there are numerous studies on the use of OCTA in ocular pathologies, repeatability of these vessel density measurements in different diseases, and more importantly, the factors affecting the repeatability of measurements have not been well studied. Initial studies which evaluated the repeatability of OCTA measured vessel densities within the ONH found the repeatability to be better than previous methods of assessing vascular parameters of the eye. ${ }^{6-8}$ Subsequent studies also evaluated the repeatability of OCTA measured vessel densities in the peripapillary $9,12,13$ and the macular regions. ${ }^{13-15}$ However, the repeatability estimates in these studies were performed predominantly in normal subjects and on small samples. The purpose of the current study was to compare the intra-session repeatability of peripapillary and macular vessel density measurements of OCTA in normal and glaucoma eyes, and to evaluate the effect of signal strength of the OCTA scans on the repeatability.

\section{METHODS}

This was a prospective, cross-sectional study conducted at Narayana Nethralaya, a tertiary eye care center in Bengaluru, South India between February 2016 and November 2016. The methodology adhered to the tenets of the Declaration of Helsinki for research involving human subjects. Written informed consent was obtained from all participants and the study was approved by the Institute's Ethics Committee. 
Participants of the study included control subjects, glaucoma suspects and glaucoma patients. Control subjects were either hospital staff or subjects who consulted for a routine eye examination or a refractive error. Control subjects had no family history of glaucoma, IOP $\leq 21 \mathrm{~mm} \mathrm{Hg}$, normal anterior and posterior segments on clinical examination by an ophthalmologist and non-glaucomatous optic discs, as assessed by glaucoma experts on masked examination of stereoscopic optic disc photographs. Glaucoma suspects either had an intraocular pressure $>21 \mathrm{mmHg}$, or suspicious $\mathrm{ONH}$ as assessed on optic disc photographs. Glaucoma patients had glaucomatous changes on $\mathrm{ONH}$ examination (focal or diffuse neuroretinal rim thinning, localized notching or retinal nerve fiber layer defects) as graded by experts on stereoscopic optic disc photographs. All types of glaucoma patients (primary or secondary, open or angle closure) were included. Inclusion criteria for all participants were age $\geq 18$ years, corrected distance visual acuity of 20/40 or better and refractive error within $\pm 5 \mathrm{D}$ sphere and $\pm 3 \mathrm{D}$ cylinder. Exclusion criteria were presence of any media opacities that prevented good quality OCT scans, or any retinal or neurological disease other than glaucoma, which could confound the evaluation. All participants underwent a comprehensive ocular examination, which included a detailed medical history, corrected distance visual acuity measurement, slit-lamp biomicroscopy, Goldmann applanation tonometry, gonioscopy, dilated fundus examination, stereoscopic disc photography, visual field (VF) examination and OCTA imaging with RTVue-XR SD-OCT (Optovue Inc., Fremont, CA).

Stereoscopic optic disc photographs were obtained by trained technicians using a digital fundus camera (Kowa nonmyd WX, Kowa Company, Ltd., Japan). Each optic disc photograph was evaluated independently by two glaucoma experts (HLR and NKP) in a masked manner to determine the presence of glaucomatous changes (focal or diffuse neuroretinal rim thinning, localized notching or RNFL defects). Discrepancy in the classification between the two experts was adjudicated by a third glaucoma expert (ZSP). Optic discs that could not be classified as normal or glaucomatous were classified as "disc suspects".

VF examination was performed using a Humphrey Field analyzer II, model 720i (Zeiss Humphrey Systems, Dublin, CA), with the Swedish interactive threshold 
algorithm (SITA) standard 24-2 program. VFs were considered reliable if the fixation losses were less than $20 \%$, and the false positive and false negative response rates were less than $15 \%$.

OCTA imaging of the optic disc region and macula was performed using RTVueXR SD-OCT (AngioVue, v2016.1.0.26). Three scans each of the optic disc and the macular region were performed in the same session by the same technician in all these subjects. The procedure of OCTA imaging with RTVue-XR has been detailed previously. ${ }^{11,16}$ The optic disc scan covers an area of $4.5 \times 4.5 \mathrm{~mm}$ and the macular scan was performed using volumetric scans covering $3 \times 3 \mathrm{~mm}$. The software compares the consecutive B-scans at the same location to detect flow using motion contrast, thereby delineating blood vessels. ${ }^{1}$ Vessel density is defined as the percentage area occupied by the large vessels and microvasculature in a particular region. Vessel densities are calculated over the entire scan area, i.e., whole enface disc and whole enface macula, as well as defined areas within each scan as described below.

In the optic disc scan, the software automatically fits an ellipse to the optic disc margin. The peripapillary region is defined as a $0.75 \mathrm{~mm}$-wide elliptical annulus extending from the optic disc boundary and the average vessel density within this region is calculated. The peripapillary vessel density was analyzed from the "Radial Peripapillary Capillary (RPC) segment" which extends from the ILM to the posterior boundary of the nerve fiber layer. The peripapillary region is divided into 6 sectors based on the Garway-Heath map and the vessel density in each sector is calculated (nasal, inferonasal, inferotemporal, superotemporal, superonasal and temporal sectors). ${ }^{17}$

Macular vessel densities are analyzed over a $1.5 \mathrm{~mm}$-wide parafoveal, circular annulus centered on the macula. Macular vessel densities analyzed in this study were of the superficial vascular plexus present in the inner layers of the retina (extending from the ILM to the inner plexiform layer). The parafoveal region is divided into 4 sectors of $90^{\circ}$ each (temporal, superior, nasal and inferior sectors) and vessel density in each sector is calculated. Image quality was assessed for all OCTA scans. Poor quality images, which were defined as those with a signal 
strength index (SSI) less than 45 or images with motion artifacts and segmentation errors were excluded from the analysis.

\section{STATISTICAL ANALYSIS}

Descriptive statistics included mean and standard deviation for normally distributed variables and median and inter-quartile range (IQR) for non-normally distributed variables.

Repeatability was assessed by intraclass correlation coefficient (ICC), withinsubject standard deviation (Sw), coefficient of repeatability (CRw) and withinsubject coefficient of variation $(\mathrm{CVw})$. The $\mathrm{Sw}$ was calculated as the square root of the within-subject mean square of error (the unbiased estimator of the component of variance due to random error) in a mixed-effects model. ${ }^{18}$ ICC was also calculated from the mixed-effects model. ${ }^{19}$ The $\mathrm{CRw}$ was calculated as 2.77 times Sw. The CVw (100 x Sw/overall mean) was calculated according to the procedure described by Bland and Altman. ${ }^{20}$ Effect of SSI on the repeatability of vessel density measurements was evaluated using linear mixed effects models for repeated measures. ${ }^{21}$ Statistical analyses were performed using the Stata version 13.1 (StataCorp, College Station, Tx) statistical software. A $p$ value of $\leq$ 0.05 was considered statistically significant.

\section{RESULTS}

One hundred and thirty-four eyes (60 normal, 31 glaucoma suspect and 43 glaucoma) of 80 subjects underwent three OCTA scans within the same session. Glaucoma suspects and glaucoma patients were considered as a single group for the analysis. Among these, 22 eyes had poor quality disc scans, 24 eyes had poor quality macular scans and 41 eyes had poor quality disc and macular scans, either in one or more of the three scans. These were excluded and the final analysis consisted of three good quality optic disc scans of 65 eyes (30 normal and 35 
glaucoma eyes) and three good quality macular scans of 69 eyes ( 35 normal and 34 glaucoma eyes). These good quality disc and macular scans were from 42 eyes of 27 normal subjects and 45 eyes of 26 glaucoma patients. Table 1 shows the demographic, clinical and visual field parameters of included subjects. Table 1 also shows the average values of the SSI and vessel densities from the three scans. Most of the vessel densities were significantly lesser in the glaucoma group compared to the control group.

Table 1. Clinical features, visual field parameters and vessel density measurements of the participants. All values represent mean \pm standard deviation unless specified.

\begin{tabular}{|c|c|c|c|}
\hline & $\begin{array}{l}\text { Control group } \\
\text { (42 eyes, } \\
27 \text { subjects) }\end{array}$ & $\begin{array}{c}\text { Glaucoma group } \\
\text { (45 eyes, } \\
26 \text { patients) }\end{array}$ & $\mathbf{P}$ \\
\hline Age (years)* & $\begin{array}{c}57.4 \\
(39.2,60.4)\end{array}$ & $\begin{array}{c}58.9 \\
(55.3,64.3)\end{array}$ & 0.23 \\
\hline Gender (male:female) & $16: 11$ & $23: 3$ & 0.02 \\
\hline Sphere (D)* & $\begin{array}{c}0.5 \\
(0,1)\end{array}$ & $\begin{array}{c}0.75 \\
(0,1.5)\end{array}$ & 0.09 \\
\hline Cylinder (D)* & $\begin{array}{l}-0.75 \\
(-1,0)\end{array}$ & $\begin{array}{c}0 \\
(-0.75,0)\end{array}$ & 0.04 \\
\hline Pseudophakia (n, \%) & $\begin{array}{c}8 \\
(19.1 \%)\end{array}$ & $\begin{array}{c}6 \\
(13.3 \%)\end{array}$ & 0.47 \\
\hline IOP at the scanning visit $(\mathrm{mm} \mathrm{Hg})$ & $14.8 \pm 2.8$ & $16.2 \pm 3.7$ & 0.06 \\
\hline Hypertension (yes:no) & $7: 20$ & $12: 14$ & 0.15 \\
\hline Diabetes mellitus (yes:no) & $4: 23$ & $13: 13$ & 0.01 \\
\hline Mean deviation $(\mathrm{dB})^{*}$ & $\begin{array}{c}-2.4 \\
(-3.7,-0.2)\end{array}$ & $\begin{array}{c}-4.7 \\
(-11.0,-2.6)\end{array}$ & $<0.001$ \\
\hline Pattern standard deviation $(\mathrm{dB})^{*}$ & $\begin{array}{c}1.8 \\
(1.4,2.6)\end{array}$ & $\begin{array}{c}3.2 \\
(1.7,9.0)\end{array}$ & $<0.001$ \\
\hline Visual field index (\%)* & $\begin{array}{c}98 \\
(97,100) \\
\end{array}$ & $\begin{array}{c}95 \\
(72,98) \\
\end{array}$ & $<0.001$ \\
\hline \multicolumn{4}{|c|}{ OCTA parameters } \\
\hline SSI (Optic disc scan) & $58.1 \pm 6.4$ & $57.1 \pm 5.7$ & 0.50 \\
\hline $\begin{array}{l}\text { Whole enface vessel density } \\
\text { (disc scan, \%) }\end{array}$ & $52.3 \pm 3.1$ & $49.3 \pm 5.4$ & 0.01 \\
\hline Average Peripapillary vessel density (\%) & $61.5 \pm 3.9$ & $58.3 \pm 5.4$ & 0.008 \\
\hline Nasal vessel density (\%) & $58.8 \pm 4.4$ & $57.0 \pm 4.1$ & 0.10 \\
\hline Inferonasal vessel density (\%) & $62.8 \pm 4.0$ & $57.4 \pm 8.1$ & 0.002 \\
\hline Inferotemporal vessel density (\%) & $65.0 \pm 4.3$ & $56.4 \pm 12.4$ & 0.001 \\
\hline Superotemporal vessel density (\%) & $65.8 \pm 4.7$ & $61.5 \pm 9.3$ & 0.03 \\
\hline Superonasal vessel density (\%) & $59.9 \pm 5.0$ & $57.0 \pm 6.9$ & 0.05 \\
\hline Temporal vessel density (\%) & $61.4 \pm 4.9$ & $60.1 \pm 4.2$ & 0.22 \\
\hline
\end{tabular}


SSI (Macula scan)

Whole enface vessel density (macula scan, \%)

Parafoveal vessel density (\%)

Temporal vessel density (\%)

Superior vessel density (\%)

Nasal vessel density (\%)

Inferior vessel density (\%)
$62.9 \pm 4.9$
$46.4 \pm 3.9$
$48.7 \pm 4.2$
$48.5 \pm 4.2$
$49.1 \pm 4.2$
$48.3 \pm 4.1$
$49.1 \pm 4.8$
$61.3 \pm 6.5$

$44.2 \pm 4.8$

$46.4 \pm 5.0$

$46.0 \pm 5.2$

$47.2 \pm 5.2$

$46.7 \pm 4.7$

$45.8 \pm 5.7$
0.27

0.04

0.04

0.03

0.11

0.13

0.01

D: diopter; dB: decibel; IOP: intraocular pressure; SSI: signal strength index; *median and interquartile range.

Table 2 shows the repeatability estimates of the peripapillary vessel density measurements separately for normal and glaucoma eyes. ICC of the inferior peripapillary measurements were greater in glaucoma compared to normal eyes. Other repeatability estimates were similar in normal and glaucoma eyes, except for the $\mathrm{CV}$ w of inferotemporal peripapillary sector which was significantly greater (worse) in the glaucoma eyes. Repeatability estimates of the peripapillary sectors were worse than that of the average and the whole enface vessel density measurement.

Table 2. Repeatability estimates of peripapillary vessel density measurements. Figures in the parenthesis represent $95 \%$ confidence limits.

\begin{tabular}{cccccccccc}
\hline \multirow{2}{*}{ Vessel density } & \multicolumn{3}{c}{ ICC } & \multicolumn{2}{c}{ Sw (\%) } & \multicolumn{2}{c}{ CRw (\%) } & \multicolumn{2}{c}{ CVw (\%) } \\
\cline { 2 - 9 } & Normal & Glaucoma & Normal & Glaucoma & Normal & Glaucoma & Normal & Glaucoma \\
\hline \multirow{2}{*}{ Whole enface disc } & 0.85 & 0.95 & 1.2 & 1.3 & 3.3 & 3.5 & 2.4 & 2.6 \\
& $(0.75,0.92)$ & $(0.91,0.97)$ & $(1.0,1.5)$ & $(1.1,1.5)$ & $(2.8,3.9)$ & $(3.0,4.1)$ & $(1.8-2.9)$ & $(2.2-3.0)$ \\
Peripapillary & 0.86 & 0.93 & 1.5 & 1.5 & 4.1 & 4.1 & 2.5 & 2.6 & $(2.5$ \\
& $(0.77,0.92)$ & $(0.87,0.96)$ & $(1.2,1.8)$ & $(1.3,1.8)$ & $(3.4,4.9)$ & $(3.5,4.8)$ & $(1.8-3.0)$ & $(2.1-3.0)$ \\
Nasal & 0.85 & 0.84 & 1.8 & 1.7 & 5.0 & 4.8 & 3.3 & 3.1 & $(2.6-3.5)$ \\
& $(0.74,0.91)$ & $(0.74,0.91)$ & $(1.5,2.2)$ & $(1.5,2.1)$ & $(4.2,6.0)$ & $(4.1,5.7)$ & $(2.4-4.0)$ & 4.1 \\
Inferonasal & 0.71 & 0.93 & 2.4 & 2.2 & 6.6 & 6.0 & 4.0 & 4.0 \\
& $(0.55,0.83)$ & $(0.88,0.96)$ & $(2.0,2.9)$ & $(1.8,2.5)$ & $(5.5,7.9)$ & $(5.1,7.0)$ & $(3.0-4.8)$ & $(3.3-4.7)$ \\
Inferotemporal & 0.75 & 0.96 & 2.3 & 2.6 & 6.5 & 7.1 & 3.7 & 6.6 \\
& $(0.59,0.85)$ & $(0.93,0.97)$ & $(2.0,2.8)$ & $(2.2,3.0)$ & $(5.4,7.7)$ & $(6.0,8.4)$ & $(2.4-4.6)$ & $(4.7-9.5)$ \\
Superotemporal & 0.85 & 0.94 & 1.9 & 2.4 & 5.3 & 6.5 & 3.0 & 4.2 \\
& $(0.75,0.92)$ & $(0.89,0.96)$ & $(1.6,2.3)$ & $(2.0,2.8)$ & $(4.4,6.3)$ & $(5.5,7.7)$ & $(2.3-3.6)$ & $(3.1-5.1)$ \\
Superonasal & 0.78 & 0.87 & 2.5 & 2.6 & 7.0 & 7.1 & 4.4 & 4.6 \\
& $(0.64,0.87)$ & $(0.79,0.93)$ & $(2.1,3.0)$ & $(2.2,3.0)$ & $(5.8,8.4)$ & $(6.0,8.4)$ & $(2.6-5.6)$ & $(3.6-5.5)$ \\
Temporal & 0.78 & 0.71 & 2.4 & 2.5 & 6.7 & 6.9 & 4.2 & 4.1 \\
& $(0.65,0.88)$ & $(0.56,0.83)$ & $(2.0,2.9)$ & $(2.1,2.9)$ & $(5.6,8.0)$ & $(5.9,8.0)$ & $(3.0-5.0)$ & $(3.3-4.8)$ \\
\hline
\end{tabular}

ICC: intraclass correlation coefficient; Sw: within subject standard deviation; CRw: coefficient of repeatability; CVw: coefficient of variation. 
Table 3 shows the repeatability estimates of the macular vessel density measurements separately in normal and glaucoma eyes. Although the repeatability estimates were slightly greater (worse) in the glaucoma eyes, the differences were not statistically significant for any of the measurements. Like the peripapillary measurements, repeatability estimates of sector measurements were worse than the average parafoveal and whole enface vessel density measurements.

Table 3. Repeatability estimates of macular vessel density measurements. Figures in the parenthesis represent $95 \%$ confidence limits.

\begin{tabular}{ccccccccc}
\hline \multirow{2}{*}{$\begin{array}{c}\text { Vessel } \\
\text { density }\end{array}$} & \multicolumn{2}{c}{ ICC } & \multicolumn{2}{c}{ Sw (\%) } & \multicolumn{2}{c}{ CRw (\%) } & \multicolumn{2}{c}{ CVw (\%) } \\
\cline { 2 - 8 } & Normal & Glaucoma & Normal & Glaucoma & Normal & Glaucoma & Normal & Glaucoma \\
\hline Whole enface & 0.87 & 0.90 & 1.5 & 1.6 & 4.1 & 4.3 & 3.3 & 3.7 \\
macula & $(0.78,0.92)$ & $0.83,0.94)$ & $(1.3,1.7)$ & $(1.3,1.9)$ & $3.5,4.8)$ & $(3.7,5.1)$ & $(2.3-4.1)$ & $(2.9-4.3)$ \\
Parafovea & 0.87 & 0.87 & 1.6 & 1.8 & 4.4 & 5.1 & 3.4 & 4.1 \\
& $(0.78,0.92)$ & $(0.79,0.93)$ & $(1.3,1.9)$ & $(1.6,2.2)$ & $(3.7,5.1)$ & $(4.3,6.0)$ & $(2.4-4.1)$ & $(3.1-4.9)$ \\
Temporal & 0.81 & 0.86 & 1.9 & 2.0 & 5.3 & 5.7 & 4.2 & 4.6 \\
& $(0.70,0.89)$ & $(0.76,0.92)$ & $(1.6,2.3)$ & $(1.7,2.4)$ & $(4.5,6.3)$ & $(4.8,6.7)$ & $(3.0-5.2)$ & $(3.7-5.3)$ \\
Superior & 0.84 & 0.85 & 1.8 & 2.1 & 4.9 & 5.9 & 3.7 & 4.7 \\
& $(0.74,0.91)$ & $(0.75,0.91)$ & $(1.5,2.1)$ & $(1.8,2.5)$ & $(4.2,5.8)$ & $(5.0,7.0)$ & $(2.8-4.5)$ & $(3.8-5.4)$ \\
Nasal & 0.80 & 0.75 & 1.9 & 2.5 & 5.4 & 6.9 & 4.2 & 5.6 \\
& $(0.69,0.88)$ & $(0.62,0.85)$ & $(1.6,2.3)$ & $(2.1,3.0)$ & $(4.5,6.3)$ & $(5.9,8.2)$ & $(3.1-5.0)$ & $(3.2-7.3)$ \\
Inferior & 0.82 & 0.86 & 2.2 & 2.2 & 6.0 & 6.1 & 4.7 & 5.0 \\
& $(0.71,0.89)$ & $(0.77,0.92)$ & $(1.8,2.6)$ & $(1.9,2.6)$ & $(5.1,7.1)$ & $(5.2,7.2)$ & $(2.6-6.1)$ & $(4.0-5.8)$ \\
\hline
\end{tabular}

ICC: intra class correlation coefficient; Sw: within subject standard deviation; CRw: coefficient of repeatability; CVw: coefficient of variation.

Table 4 shows the effect of SSI on the repeatability of vessel density measurements. The significant positive coefficients associated with SSI indicated that the vessel density significantly increased with an increase in the SSI values of the repeat scans.

Table 4. Effect of signal strength index on the repeatability of vessel density measurements of the peripapillary and macular regions.

\begin{tabular}{lccc}
\hline \multicolumn{1}{c}{ Vessel density } & Coefficient (SE) & 95\% Cl & P value \\
\hline Whole enface disc & $0.21(0.03)$ & $0.15-0.26$ & $<0.001$ \\
Peripapillary & $0.23(0.03)$ & $0.17-0.29$ & $<0.001$ \\
$\quad$ Nasal & $0.25(0.04)$ & $0.17-0.32$ & $<0.001$ \\
$\quad$ Inferonasal & $0.19(0.05)$ & $0.08-0.29$ & 0.001 \\
Inferotemporal & $0.20(0.06)$ & $0.07-0.32$ & 0.002 \\
Superotemporal & $0.15(0.05)$ & $0.05-0.25$ & 0.004
\end{tabular}




$\begin{array}{llll}\text { Superonasal } & 0.21(0.06) & 0.09-0.33 & 0.001 \\ \text { Temporal } & 0.35(0.05) & 0.26-0.45 & <0.001 \\ \text { Whole enface macula } & 0.28(0.04) & 0.20-0.35 & <0.001 \\ \text { Parafovea } & 0.31(0.04) & 0.23-0.39 & <0.001 \\ \quad \text { Temporal } & 0.30(0.05) & 0.20-0.39 & <0.001 \\ \text { Superior } & 0.29(0.05) & 0.20-0.39 & <0.001 \\ \text { Nasal } & 0.38(0.05) & 0.29-0.47 & <0.001 \\ \text { Inferior } & 0.36(0.05) & 0.26-0.46 & <0.001\end{array}$

$\mathrm{SE}$ : standard error; $\mathrm{Cl}$ : confidence interval.

\section{DISCUSSION}

The present study evaluated the repeatability of OCTA-measured peripapillary and macular vessel densities and found that the repeatability estimates were similar in normal and glaucoma eyes. Few studies have evaluated the repeatability of OCTA measured vessel densities in the peripapillary $9,12,13$ and the macular regions. ${ }^{13-15}$ However, the repeatability estimates in these studies were performed predominantly in normal subjects and on small samples. CVw was the only repeatability parameter estimated in these studies and, in most of the studies, repeatability was estimated only for the average vessel density measurements and not for sectors.

Wang et al evaluated the repeatability of average peripapillary vessel density in 15 normal eyes and reported a CVw of $1.21 \% .{ }^{13}$ Liu et al evaluated the repeatability of average peripapillary vessel density in 12 normal eyes and 12 glaucoma eyes and reported a $\mathrm{CVw}$ of $1.9 \%$ and $4 \%$ respectively. ${ }^{9}$ Unlike the previous studies which evaluated only the average peripapillary measurement, Hollo evaluated the repeatability of vessel density measurements of various peripapillary sectors in 18 glaucoma eyes (8 glaucoma and 10 ocular hypertensive eyes) and found that the $\mathrm{CVw}$ ranged from $3.51 \%$ (temporal sector measurement) to $5.12 \%$ (superotemporal sector measurement). ${ }^{12}$ Summarizing the results from the previous studies, it seemed that the CVw of peripapillary vessel density was better in normal compared to the glaucoma eyes, and the repeatability varied across different peripapillary sectors. However, in the current study, we found that the 
repeatability of peripapillary vessel densities were statistically similar in normal and glaucoma eyes, except for the CVw of inferotemporal sector measurement which was significantly worse in the glaucoma eyes. One possible reason for similar repeatability estimates in normal and glaucoma eyes in our study is that the severity of disease was mild in most of the glaucoma eyes and suspect eyes, which had normal VF, were also included in the glaucoma group. Similar to the findings of the study by Hollo, ${ }^{12}$ we too found that the repeatability varied across different peripapillary sectors.

Yu et al evaluated the agreement (instead of repeatability) between two repeated measurements of average parafoveal vessel density in 15 normal eyes and reported that the $95 \%$ limits of agreement ranged between $-8 \%$ and $11 \%{ }^{14}$ Agemy et al evaluated the repeatability of average parafoveal vessel density in the superficial retinal, deep retinal and choroidal layers of 5 normal eyes and reported a $\mathrm{CVw}$ ranging from $0.1 \%$ to $6.8 \%$; without reporting the $\mathrm{CVw}$ of different layers separately. ${ }^{15}$ Wang et al evaluated the repeatability of average parafoveal vessel density in 15 normal eyes and reported a CVw of $4.55 \% .{ }^{13}$ This is similar to the $\mathrm{CVw}$ values found in normal eyes of our study. There are, however, no reports on the repeatability of macular vessel densities in eyes with glaucoma.

In addition to $\mathrm{CVw}$, we also evaluated $\mathrm{CRw}$ as an estimate of repeatability. Unlike CVw, CRw has greater relevance for clinicians as it represents the testretest variability of the measurements. ${ }^{18} \mathrm{CRw}$ values of the most important peripapillary sectors (inferotemporal and superotemporal) were close to $7 \%$. CRw of the parafoveal vessel densities also ranged between $4 \%$ and $7 \%$. This would mean that any change in the peripapillary and parafoveal vessel density of less than $7 \%$ would fall within the test-retest variability and would be clinically insignificant. This has to be considered while interpreting any change in vessel densities longitudinally.

We evaluated the effect of SSI of the OCTA scans on the repeatability of vessel densities and found a significant positive association between the two. The vessel densities both in peripapillary and macular regions significantly increased with an increase in the SSI values of repeat scans. The coefficients ranged from 0.15 
to 0.38 for peripapillary vessel densities which meant that the vessel densities increased by $1.5 \%$ to $3.8 \%$ if the SSI value of the repeat scan increased by 10 units. The coefficients were larger for the association between SSI and macular vessel densities and ranged from 0.28 to 0.38 . Variability in SSI values of the repeat scans is likely to explain a significant part of the repeatability estimates seen in the present and previous studies. Therefore, SSI has to be considered while interpreting the changes in vessel density longitudinally.

There are some limitations of the OCTA technology which need to be considered while interpreting the results. A significant number of OCTA scans were excluded because of poor quality, which was either due to low SSI or residual motion artifacts. Some of the previous studies have also reported high numbers of poor quality images with OCTA. ${ }^{12,22,23}$ Real time tracking is used in the current versions of the OCTA technology to reduce artifacts. ${ }^{24}$ The OCTA algorithm, in its current form, includes large vessels along with capillaries in its estimation of vessel density. It is possible that the repeatability estimates are different for the measurement of large vessels compared to that of the capillaries. The peripapillary vessel densities can also be affected by parapapillary atrophy (PPA). ${ }^{25}$ We did not record the presence of PPA or its extent in our subjects. However, the number of eyes with PPA in our study is unlikely to be significant as we had excluded high myopic subjects. Also, our results apply to OCTA imaging performed with RTVue and caution should be exercised while extrapolating the results to OCTA devices which use different platforms and algorithms for imaging blood vessels.

In conclusion, repeatability estimates of OCTA measured peripapillary and macular vessel densities were similar in normal and glaucomatous eyes. Changes in the peripapillary and parafoveal vessel density of less than $7 \%$ fall within the test-retest variability of the OCTA vessel density measurements and are therefore likely to be clinically insignificant. SSI values of the scans had a significant effect on the repeatability of OCTA measurements with the vessel density values increasing in scans with higher SSI values. 


\section{REFERENCES}

1. Jia Y, Tan O, Tokayer J, et al. Split-spectrum amplitude-decorrelation angiography with optical coherence tomography. Opt Express 2012;20:4710-4725.

2. Ishibazawa A, Nagaoka T, Takahashi A, et al. Optical Coherence Tomography Angiography in Diabetic Retinopathy: A Prospective Pilot Study. Am J Ophthalmol 2015;160:35-44.

3. Jia Y, Bailey ST, Wilson DJ, et al. Quantitative optical coherence tomography angiography of choroidal neovascularization in age-related macular degeneration. Ophthalmology 2014;121:1435-1444.

4. Kuehlewein L, Bansal M, Lenis TL, et al. Optical Coherence Tomography Angiography of Type 1 Neovascularization in Age-Related Macular Degeneration. Am J Ophthalmol 2015;160:739748.

5. Coscas F, Glacet-Bernard A, Miere A, et al. Optical Coherence Tomography Angiography in Retinal Vein Occlusion: Evaluation of Superficial and Deep Capillary Plexa. Am J Ophthalmol 2016;161:160-171.

6. Jia Y, Morrison JC, Tokayer J, et al. Quantitative OCT angiography of optic nerve head blood flow. Biomed Opt Express 2012;3:3127-3137.

7. Jia $Y$, Wei $E$, Wang $X$, et al: Optical coherence tomography angiography of optic disc perfusion in glaucoma. Ophthalmology 2014;121:1322-1332.

8. Wang $\mathrm{X}$, Jiang $\mathrm{C}$, Ko $\mathrm{T}$, et al. Correlation between optic disc perfusion and glaucomatous severity in patients with open-angle glaucoma: an optical coherence tomography angiography study. Graefes Arch Clin Exp Ophthalmol 2015;253:1557-1564.

9. Liu L, Jia Y, Takusagawa HL, et al. Optical Coherence Tomography Angiography of the Peripapillary Retina in Glaucoma. JAMA Ophthalmol 2015;133:1045-1052.

10. Yarmohammadi A, Zangwill LM, Diniz-Filho A, et al. Optical Coherence Tomography Angiography Vessel Density in Healthy, Glaucoma Suspect, and Glaucoma Eyes. Invest Ophthalmol Vis Sci 2016;57:OCT451-459.

11. Rao HL, Pradhan ZS, Weinreb RN, et al. Regional Comparisons of Optical Coherence Tomography Angiography Vessel Density in Primary Open-Angle Glaucoma. Am J Ophthalmol 2016;171:75-83.

12. Hollo G: Intrasession and Between-Visit Variability of Sector Peripapillary Angioflow Vessel Density Values Measured with the Angiovue Optical Coherence Tomograph in Different Retinal Layers in Ocular Hypertension and Glaucoma. PLoS One 2016;11:e0161631.

13. Wang $X$, Kong $X$, Jiang $C$, et al. Is the peripapillary retinal perfusion related to myopia in healthy eyes? A prospective comparative study. BMJ Open 2016;6:e010791.

14. Yu J, Jiang C, Wang $X$, et al. Macular perfusion in healthy Chinese: an optical coherence tomography angiogram study. Invest Ophthalmol Vis Sci 2015;56:3212-3217.

15. Agemy SA, Scripsema NK, Shah CM, et al. Retinal Vascular Perfusion Density Mapping Using Optical Coherence Tomography Angiography in Normals and Diabetic Retinopathy Patients. Retina 2015;35:2353-2363. 
16. Rao HL, Pradhan ZS, Weinreb RN, et al. Vessel density and structural measurements of optical coherence tomography in primary angle closure and primary angle closure glaucoma. Am J Ophthalmol 2017;177:106-115.

17. Garway-Heath DF, Poinoosawmy D, Fitzke FW, et al. Mapping the visual field to the optic disc in normal tension glaucoma eyes. Ophthalmology 2000;107:1809-1815.

18. Bland JM, Altman DG: Measurement error. BMJ 1996;313:744.

19. Shrout PE, Fleiss JL: Intraclass correlations: uses in assessing rater reliability. Psychol Bull 1979;86:420-428.

20. Bland JM, Altman DG: Measurement error proportional to the mean. BMJ 1996;313:106.

21. West BT, Welch KB, Galecki AT: Linear mixed models: a practical guide using statistical software. Boca Raton: CRC Press; 2007.

22. Suh MH, Zangwill LM, Manalastas PI, et al. Optical Coherence Tomography Angiography Vessel Density in Glaucomatous Eyes with Focal Lamina Cribrosa Defects. Ophthalmology 2016;123:2309-2317.

23. Spaide RF, Fujimoto JG, Waheed NK: Image Artifacts in Optical Coherence Tomography Angiography. Retina 2015;35:2163-2180.

24. Camino A, Zhang M, Gao SS, et al. Evaluation of artifact reduction in optical coherence tomography angiography with real-time tracking and motion correction technology. Biomed Opt Express 2016;7:3905-3915.

25. Akagi T, lida Y, Nakanishi H, et al. Microvascular Density in Glaucomatous Eyes With Hemifield Visual Field Defects: An Optical Coherence Tomography Angiography Study. Am J Ophthalmol 2016;168:237-249. 


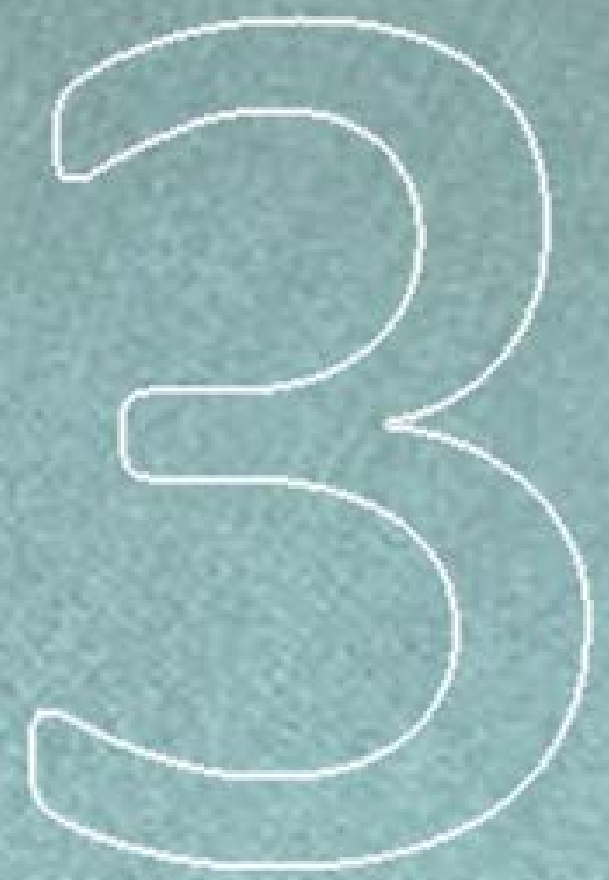

\section{Determinants of peripapillary and macular vessel densities in normal eyes}

Determinants of peripapillary and macular vessel densities measured by optical coherence tomography angiography in normal eyes

Harsha L Rao, Zia S Pradhan, Robert N Weinreb, Hemanth B Reddy, Mohammed Riyazuddin, Sonia Sachdeva, Narendra K Puttaiah, Chaitra Jayadev, Carroll AB Webers.

J Glaucoma 2017 May;26(5):491-497 


\section{ABSTRACT}

Purpose: To evaluate the effect of subject-related (age, gender, systemic hypertension and diabetes), eye-related (refractive error, optic disc size) and technology-related (signal strength index, SSI of the scans) determinants on the peripapillary and macular vessel densities measured with optical coherence tomography angiography (OCTA) in normal eyes.

Methods: In a cross-sectional study, 181 normal eyes of 107 subjects (45 men, 62 women, median age: 50 years, range: $18-77$ years) underwent OCTA imaging. Linear mixed models were used to analyze the effect of the determinants on the peripapillary and macular vessel densities of OCTA.

Results: Age and optic disc size did not affect the vessel densities of any of the regions ( $p>0.05$ for all associations). En face optic disc (coefficient: $1.67, p<0.001$ ) and most of the peripapillary vessel densities were higher in females. En face disc (coefficient $=-1.88, p=0.02$ ) and most of the peripapillary vessel densities were lower, while the parafoveal vessel density was higher, (coefficient $=2.32$, $p=0.01$ ) in subjects with hypertension. Most of the vessel densities were lower in subjects with diabetes. SSI showed a statistically significant association with the vessel densities of all regions (coefficients: 0.14 to 0.27 for peripapillary and 0.20 to 0.27 for macular sectors).

Conclusions: Most of the peripapillary vessel densities were higher in females. Hypertension and diabetes also affected the vessel densities. Vessel densities in all the regions were significantly higher in scans with higher SSI. These results should be considered while interpreting the vessel densities in retinal diseases and glaucoma. 


\section{INTRODUCTION}

A recent application of optical coherence tomography (OCT) has been the development of a three-dimensional angiography algorithm called split spectrum amplitude-decorrelation angiography (SSADA) for imaging the retinal and optic nerve head (ONH) microcirculation non-invasively. ${ }^{1}$ Multiple studies have used OCT angiography (OCTA) to report the vascular changes in common retinal pathologies such as diabetic retinopathy, ${ }^{2}$ age related macular degeneration ${ }^{3,4}$ and retinal vein occlusions. ${ }^{5}$ OCTA has also been used to demonstrate reduced $\mathrm{ONH}$ and peripapillary vessel densities in patients with glaucoma. ${ }^{6-12}$ Additionally, several studies have reported good intra- and inter-visit repeatability of the vascular measurements of OCTA. ${ }^{6-9,13-15}$ Although there are numerous studies on the use of OCTA in ocular pathologies, literature on the determinants of retinal and ONH perfusion measurements provided by OCTA in normal subjects is sparse. Yu et al evaluated the effect of age and gender on the vessel density measurements of OCTA at the macula in healthy Chinese subjects and found that the parafoveal vessel density decreased significantly with increasing age and the decrease was greater in males as compared to females. ${ }^{14}$ In contrast, another study found no effect of age on the parafoveal vessel density measurements in healthy Indian subjects. ${ }^{16}$ Wang et al. evaluated the effect of myopia on the peripapillary and parafoveal OCTA measurements and found a significant reduction of vessel density and blood flow index in the peripapillary but not the parafoveal region of high myopia eyes compared to emmetropic eyes. ${ }^{15}$ These previous studies were restricted to evaluating the effect of age, gender and refractive error on the OCTA measurements. The purpose of the current study was to evaluate the effect of subject-related (age, gender, presence of systemic hypertension and diabetes), eye-related (refractive error, optic disc size) and technology-related (signal strength of the scans) factors on the $\mathrm{ONH}$, peripapillary and macular vessel densities measured with OCTA in normal subjects. 


\section{METHODS}

This was a prospective, cross-sectional study conducted at Narayana Nethralaya, a tertiary eye care center in Bengaluru, South India between September 2015 and March 2016. The methodology adhered to the tenets of the Declaration of Helsinki for research involving human subjects. Written informed consent was obtained from all participants and the study was approved by the Institute's Ethics Committee.

Participants of the study included normal subjects, who were either hospital staff or subjects who consulted for a routine eye examination or a refractive error. These subjects had no family history of glaucoma, IOP $\leq 21 \mathrm{~mm} \mathrm{Hg}$, open angles on gonioscopy, and normal anterior and posterior segment in the included eye as determined by an ophthalmologist. The other inclusion criteria were age $\geq 18$ years, corrected distance visual acuity of $20 / 40$ or better and refractive error within $\pm 5 \mathrm{D}$ sphere and $\pm 3 \mathrm{D}$ cylinder. Eyes with suspicious findings of glaucoma (cup to disc ratio asymmetry between the two eyes, adjusted for the disc size, of greater than 0.2 , cup to disc ratio greater than 0.7 , neuroretinal rim narrowing, notching or retinal nerve fiber layer defects) were excluded. Eyes with media opacities that prevented good quality OCT scans, and any retinal or neurological disease were excluded. Eyes with a past history of trauma or inflammation were also excluded. All participants underwent a comprehensive ocular examination, which included a detailed medical history (including a selfreported history of diabetes and systemic hypertension), corrected distance visual acuity measurement, slit-lamp biomicroscopy, Goldmann applanation tonometry, gonioscopy, dilated fundus examination and OCTA imaging with RTVue-XR SD-OCT (Optovue Inc., Fremont, CA).

OCTA imaging of the optic nerve head (ONH) and macula was performed using RTVue-XR SD-OCT (AngioVue, v2015.100.0.33). Details of the imaging procedure has been described previously. ${ }^{12}$ In brief, RTVue-XR uses an $840 \mathrm{~nm}$ diode laser source, with an A-scan rate of $70 \mathrm{kHz}$ per second. Optic disc and macular imaging is performed using a set of 2 scans; one vertical priority and one horizontal 
priority raster volumetric scan. The optic disc scan covers an area of $4.5 \times 4.5$ $\mathrm{mm}$ (Figure 1a) and the macular scan (Figure 1c) was performed using volumetric scans covering $3 \times 3 \mathrm{~mm}$. An orthogonal registration algorithm is used to produce merged 3-dimensional OCT angiograms. ${ }^{17}$ The SSADA algorithm compares the consecutive B-scans at the same location to detect flow using motion contrast. ${ }^{1}$ Vessel densities are calculated over the entire scan area, i.e., whole en face disc and whole en face macula. Vessel density is defined as the percentage area occupied by the large vessels and microvasculature in a particular region. In addition to the whole scan analysis, the software calculates vessel densities in various layers of the retina and the $\mathrm{ONH}$, and each scan region is further divided into sectors as described below. An en face angiogram of the $\mathrm{ONH}$ and peripapillary circulation is obtained by the maximum flow (decorrelation value) projection from the inner limiting membrane (ILM) to retinal pigment epithelium (RPE). The software automatically fits an ellipse to the optic disc margin and defines the peripapillary region as a $0.75 \mathrm{~mm}$-wide elliptical annulus extending from the optic disc boundary (Figure 1 b). The peripapillary vessels were analyzed in superficial retinal layers from the Radial Peripapillary Capillary (RPC) segment. RPC segment extends from the ILM to the nerve fiber layer. The peripapillary region was divided into 6 sectors based on the Garway-Heath map (Figure 1b) and vessel densities for the entire peripapillary area (average) and each sector were determined. ${ }^{18}$ Macular vessel densities analyzed in this study included the superficial vascular plexus present in the inner layers of the retina (extending from the internal limiting membrane to the inner plexiform layer). Macular vessel densities were analyzed over a $1.5 \mathrm{~mm}$-wide parafoveal, circular annulus centered on the macula (Figure 1d). The parafoveal region was also divided into 4 sectors of $90^{\circ}$ each (nasal, inferior, superior and temporal sectors). Image quality was assessed for all OCTA scans. Poor quality images, defined as scans with a signal strength index (SSI) less than 35 or images with residual motion artifacts were excluded from the analysis. 
(a)

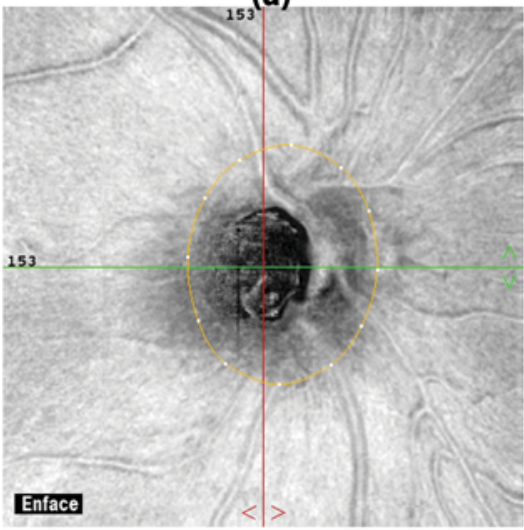

(c)

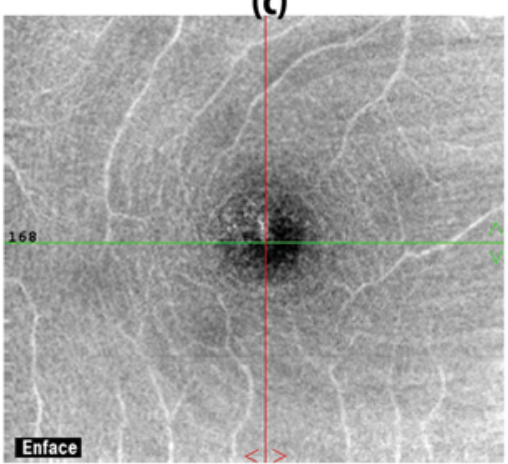

(b)

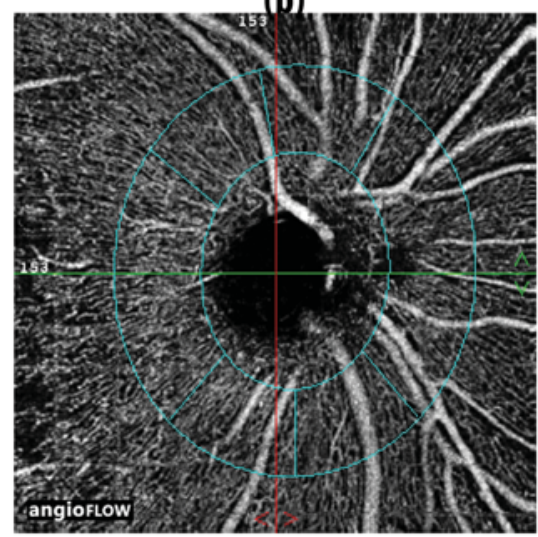

(d)

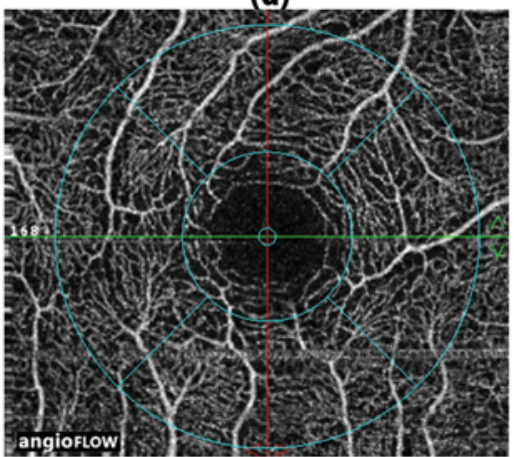

Figure 1. Figure showing the (a) en face and (b) optical coherence tomography angiography image of the peripapillary and (c) en face and (d) optical coherence tomography angiography images of the macular region and the sectors where vessel densities are calculated. The whole en face disc and macular vessel density are calculated over entire scan area. The peripapillary vessel density is calculated over a $0.75 \mathrm{~mm}$-wide elliptical annulus extending from the optic disc boundary from the radial peripapillary capillary segment and the superficial macular vessel density over a $1.5 \mathrm{~mm}$-wide circular annulus centered on the macula.

A subset of the subjects also underwent optic disc area measurement on RTVueXR SD-OCT using the traditional ONH scan. The ONH scan consists of 12 radial scans $3.4 \mathrm{~mm}$ in length and 6 concentric ring scans ranging from 2.5 to $4.0 \mathrm{~mm}$ in diameter all centered on the optic disc. RPE tips are automatically detected by the software and are joined to delineate the optic disc margin for calculation of the disc area. Position of the RPE tips were corrected manually by the operator 
if the automated detection was found to be inaccurate. All the examinations for a particular subject were performed on the same day.

\section{STATISTICAL ANALYSIS}

Descriptive statistics included mean and standard deviation for normally distributed variables, and median and interquartile range (IQR) for non-normally distributed variables. Linear mixed models were fit to assess the effects of age, gender, mean refractive spherical equivalent (MRSE), optic disc area, hypertension, diabetes mellitus and SSI on the peripapillary and macular vessel densities measured by OCTA. Linear mixed model is a parametric linear model which quantifies the relationship between a continuous dependent variable and one or more independent (determinant) variables, specifically used with clustered, longitudinal or repeated measures data. ${ }^{19}$ It can include fixed and random effect-parameters, accounting for the correlation among random effect-parameters. While the fixed effect-parameters describe the relationship between the independent and the dependent variable for the entire cohort, random effect-parameters describe the relationship specifically for the clusters within the cohort. Vessel density parameters were considered as dependent variables and age, gender, MRSE, optic disc area, presence of hypertension and diabetes, and SSI were considered as determinant variables. Collinearity among the determinant variables was assessed using correlation analysis and variables with a correlation coefficient ( $r$ ) of $\geq 0.50$ were considered to be strongly correlated with each other. All other assumptions for linear mixed modeling analysis were checked for before fitting the models. All determinant variables were treated as fixed effect-parameters and the subject was treated as a random effect-parameter in the mixed models. As the optic disc area measurements were available only in a few subjects, two separate models were built for each dependent variable, one excluding the disc area and one including the disc area as a determinant. Coefficient associated with disc area was derived from the multivariate model that contained it as a determinant variable while the coefficients associated with the rest of the determinant variables were derived from the model that excluded disc area (to 
provide the benefit of the larger sample size). Statistical analyses were performed using commercial software (Stata ver. 13.1; StataCorp, College Station, TX). A p value of $\leq 0.05$ was considered statically significant.

\section{RESULTS}

One hundred and eighty-seven eyes of 108 subjects underwent OCTA imaging with SD-OCT. Among these, 10 eyes with a poor quality disc scan, 24 eyes with a poor quality macular scan and 6 eyes with both poor quality disc and macular scans were excluded. Final analysis included 171 optic disc scans (104 subjects) and 157 macular scans (100 subjects) from 181 eyes of 107 subjects. Table 1 shows the demographic, clinical and vessel density parameters of included subjects. Of the 16 subjects with hypertension, 7 also had diabetes. Ten eyes, which were pseudophakic were excluded from MRSE analysis. Peripapillary vessel density was greatest in the inferotemporal and the superotemporal quadrants. Parafoveal vessel densities were greater in the inferior and the superior quadrants compared to the nasal quadrant.

Table 1. Clinical and optical coherence tomography angiography characteristics of the participants.

\begin{tabular}{lcc}
\hline & $\begin{array}{c}\text { Median } \\
\text { (Interquartile range) }\end{array}$ & Range \\
\hline Age (years) & $50(38,58)$ & $18-77$ \\
Gender (male:female) & $45: 62$ & \\
Sphere (D) & $0(0,0.75)$ & -4.0 to +3.0 \\
Cylinder (D) & $-0.5(-0.75,0)$ & -3.0 to 0 \\
MRSE (D) & $0(-0.38,0.38)$ & -4.0 to 2.75 \\
Hypertension ( $\mathrm{n}, \%)$ & $16(15.0 \%)$ & \\
Diabetes mellitus (n, \%) & $16(15.0 \%)$ & \\
Optic disc area (mm $\left.{ }^{2}, \mathrm{n}=76\right)$ & $2.3(2.0,2.6)$ & 1.5 to 3.6 \\
Intraocular pressure (mm Hg) & 14 & 10 to 20 \\
SSI (optic disc scan) & $57(51,65)$ & 35 to 76 \\
Whole en face vessel density (disc scan) & $54.8(52.5,57.1)$ & 45.7 to 61.0
\end{tabular}




$\begin{array}{llr}\text { Average Peripapillary vessel density (\%) } & 62.7(60.4,65.2) & 52.7 \text { to } 71.6 \\ \text { Nasal vessel density (\%) } & 61.1(57.7,63.4) & 46.9 \text { to } 68.1 \\ \text { Inferonasal vessel density (\%) } & 63.7(61.0,67.2) & 49.2 \text { to } 73.8 \\ \text { Inferotemporal vessel density (\%) } & 66.8(63.8,69.6) & 49.5 \text { to } 74.7 \\ \text { Superotemporal vessel density (\%) } & 66.8(64.0,69.4) & 51.3 \text { to } 76.4 \\ \text { Superonasal vessel density (\%) } & 62.6(58.1,65.9) & 45.5 \text { to } 73.2 \\ \text { Temporal vessel density (\%) } & 61.9(58.6,64.8) & 45.7 \text { to } 75.7 \\ \text { SSI (Macula scan) } & 65(61,69) & 45 \text { to } 77 \\ \text { Whole en face vessel density (macula scan) } & 48.9(46.3,50.7) & 40.0 \text { to } 56.2 \\ \text { Foveal vessel density (\%) } & 26.2(22.5,28.9) & 14.6 \text { to } 37.1 \\ \text { Parafoveal vessel density (\%) } & 50.4(47.9,52.9) & 41.7 \text { to } 58.4 \\ \text { Nasal vessel density (\%) } & 48.9(46.9,51.4) & 38.7 \text { to } 57.6 \\ \text { Inferior vessel density (\%) } & 51.5(48.2,54.1) & 40.2 \text { to } 59.5 \\ \text { Superior vessel density (\%) } & 51.5(48.7,54.0) & 38.4 \text { to } 59.5 \\ \text { Temporal vessel density (\%) } & 50.0(47.5,52.2) & 40.3 \text { to } 58.3\end{array}$

MRSE: mean refractive spherical equivalent; SSI: signal strength index.

We evaluated the correlations between the determinant variables. Males were statistically significantly older than females $(r=0.19, p=0.01)$. Subjects with hypertension $(r=0.42, p<0.001)$ and diabetes $(r=0.35, p<0.001)$ were significantly older than those without hypertension and diabetes. Disc size was larger in younger subjects $(r=-0.47, p<0.001)$. SSI of both the optic disc $(r=-0.24, p=0.002)$ and the macular $(r=-0.36, p<0.001)$ scan were lower in older subjects. SSI of the macular scan was lower in subjects with hypertension $(r=-0.19, p=0.02)$ and diabetes $(r=-0.23, p=0.003)$. As none of the determinant variables showed strong correlations with each other $(r>0.50)$, all these variables were introduced into the multivariate linear mixed models.

Table 2 shows the effect of the determinant variables on the vessel densities of the en face $\mathrm{ONH}$ scan and the peripapillary sectors. The vessel density of the disc scan was significantly greater in women, in scans with higher SSI and was significantly lesser in eyes of subjects with hypertension. The average and most of the sector-wise peripapillary vessel densities were greater in females compared to males. Peripapillary vessel densities were lower in subjects with hypertension 
and diabetes. However, this association was statistically significant only in the superonasal sector for subjects with hypertension. SSI showed a statistically significant positive association with all peripapillary vessel density measurements with vessel densities being significantly greater in scans with higher SSIs (Figure 2a).

Table 2. Effect of determinant variables on the whole en face optic nerve head and peripapillary vessel density measurements. Number in the cell represents coefficient value with the $p$ value in parenthesis.

\begin{tabular}{lccccccc}
\hline Vessel density & Age & Female & MRSE & Disc area & Hypertension & Diabetes & SSI \\
\hline Whole en face & -0.01 & 1.67 & 0.31 & -1.52 & -1.88 & -0.92 & 0.19 \\
& $(0.21)$ & $(<0.001)$ & $(0.11)$ & $(0.07)$ & $(0.02)$ & $(0.23)$ & $(<0.001)$ \\
Average & -0.02 & 1.23 & 0.18 & 0.05 & -1.23 & -0.78 & 0.20 \\
peripapillary & $(0.40)$ & $(0.02)$ & $(0.38)$ & $(0.95)$ & $(0.13)$ & $(0.32)$ & $(<0.001)$ \\
Nasal & -0.01 & 1.71 & 0.16 & -0.47 & -1.45 & -1.50 & 0.21 \\
& $(0.86)$ & $(0.01)$ & $(0.53)$ & $(0.67)$ & $(0.14)$ & $(0.12)$ & $(<0.001)$ \\
Inferonasal & -0.02 & 0.18 & 0.20 & -1.06 & -0.28 & -1.38 & 0.16 \\
& $(0.52)$ & $(0.83)$ & $(0.56)$ & $(0.49)$ & $(0.83)$ & $(0.27)$ & $(<0.001)$ \\
Inferotemporal & -0.02 & 1.75 & 0.55 & 0.85 & -0.08 & -0.56 & 0.14 \\
& $(0.58)$ & $(0.02)$ & $(0.07)$ & $(0.51)$ & $(0.95)$ & $(0.62)$ & $(<0.001)$ \\
Superotemporal & 0.003 & 1.24 & -0.44 & 1.53 & -0.88 & -1.13 & 0.14 \\
& $(0.93)$ & $(0.13)$ & $(0.16)$ & $(0.25)$ & $(0.46)$ & $(0.33)$ & $(0.001)$ \\
Superonasal & 0.01 & 1.40 & -0.01 & -1.27 & -2.93 & -1.74 & 0.18 \\
& $(0.75)$ & $(0.12)$ & $(0.97)$ & $(0.21)$ & $(0.03)$ & $(0.10)$ & $(<0.001)$ \\
Temporal & -0.03 & 1.23 & 0.24 & 1.76 & -1.25 & 0.99 & 0.27 \\
& $(0.23)$ & $(0.12)$ & $(0.41)$ & $(0.17)$ & $(0.27)$ & $(0.37)$ & $(<0.001)$ \\
\hline
\end{tabular}

MRSE: mean refractive spherical equivalent; SSI: signal strength index.

Table 3 shows the effect of the determinant variables on the macular vessel densities. Macular vessel densities were higher in subjects with hypertension and this association was statistically significant for the average, nasal and temporal sector parafoveal measurements. Macular vessel densities were lower in subjects with diabetes but this association was statistically significant only for the temporal parafoveal measurement. SSI showed a statistically significant positive association with all parafoveal vessel density measurements with vessel densities being significantly greater in scans with higher SSIs (Figure 2b). 


\section{Chapter 3}

Table 3. Effect of determinant variables on the macular vessel density measurements. Number in the cell represents coefficient value with the $p$ value in parenthesis.

\begin{tabular}{|c|c|c|c|c|c|c|c|}
\hline Vessel density & Age & Female & MRSE & Disc area & Hypertension & Diabetes & SSI \\
\hline Whole en face & $\begin{array}{c}0.01 \\
(0.77)\end{array}$ & $\begin{array}{l}-0.61 \\
(0.21)\end{array}$ & $\begin{array}{c}0.07 \\
(0.75)\end{array}$ & $\begin{array}{c}0.01 \\
(0.99)\end{array}$ & $\begin{array}{c}1.56 \\
(0.08)\end{array}$ & $\begin{array}{l}-1.59 \\
(0.06)\end{array}$ & $\begin{array}{c}0.27 \\
(<0.001)\end{array}$ \\
\hline Foveal & $\begin{array}{c}0.02 \\
(0.50)\end{array}$ & $\begin{array}{l}-0.56 \\
(0.54)\end{array}$ & $\begin{array}{l}-0.22 \\
(0.56)\end{array}$ & $\begin{array}{c}1.46 \\
(0.21)\end{array}$ & $\begin{array}{l}-0.60 \\
(0.67)\end{array}$ & $\begin{array}{c}1.22 \\
(0.41)\end{array}$ & $0.11(0.04)$ \\
\hline Parafoveal & $\begin{array}{l}-0.01 \\
(0.73)\end{array}$ & $\begin{array}{l}-0.64 \\
(0.21)\end{array}$ & $\begin{array}{l}-0.07 \\
(0.77)\end{array}$ & $\begin{array}{c}0.07 \\
(0.94)\end{array}$ & $\begin{array}{c}2.32 \\
(0.01)\end{array}$ & $\begin{array}{l}-1.61 \\
(0.06)\end{array}$ & $\begin{array}{c}0.23 \\
(<0.001)\end{array}$ \\
\hline Nasal & $\begin{array}{l}-0.03 \\
(0.15)\end{array}$ & $\begin{array}{l}-1.00 \\
(0.06)\end{array}$ & $\begin{array}{c}0.28 \\
(0.26)\end{array}$ & $\begin{array}{c}1.13 \\
(0.24)\end{array}$ & $\begin{array}{c}2.19 \\
(0.01)\end{array}$ & $\begin{array}{l}-1.13 \\
(0.27)\end{array}$ & $\begin{array}{c}0.20 \\
(<0.001)\end{array}$ \\
\hline Inferior & $\begin{array}{l}0.004 \\
(0.87)\end{array}$ & $\begin{array}{l}-0.48 \\
(0.42)\end{array}$ & $\begin{array}{l}-0.27 \\
(0.32)\end{array}$ & $\begin{array}{l}-0.90 \\
(0.37)\end{array}$ & $\begin{array}{c}1.42 \\
(0.18)\end{array}$ & $\begin{array}{l}-1.67 \\
(0.10)\end{array}$ & $\begin{array}{c}0.25 \\
(<0.001)\end{array}$ \\
\hline Superior & $\begin{array}{c}-0.01 \\
(0.61)\end{array}$ & $\begin{array}{l}-0.47 \\
(0.45)\end{array}$ & $\begin{array}{l}-0.20 \\
(0.48)\end{array}$ & $\begin{array}{c}0.33 \\
(0.74)\end{array}$ & $\begin{array}{c}1.90 \\
(0.10)\end{array}$ & $\begin{array}{l}-1.57 \\
(0.17)\end{array}$ & $\begin{array}{c}0.27 \\
(<0.001)\end{array}$ \\
\hline Temporal & $\begin{array}{c}0.01 \\
(0.71) \\
\end{array}$ & $\begin{array}{l}-0.55 \\
(0.33) \\
\end{array}$ & $\begin{array}{c}-0.09 \\
(0.71) \\
\end{array}$ & $\begin{array}{c}0.20 \\
(0.84) \\
\end{array}$ & $\begin{array}{c}3.02 \\
(0.01) \\
\end{array}$ & $\begin{array}{l}-2.48 \\
(0.01) \\
\end{array}$ & $\begin{array}{c}0.21 \\
(<0.001) \\
\end{array}$ \\
\hline
\end{tabular}

MRSE: mean refractive spherical equivalent; SSI: signal strength index.
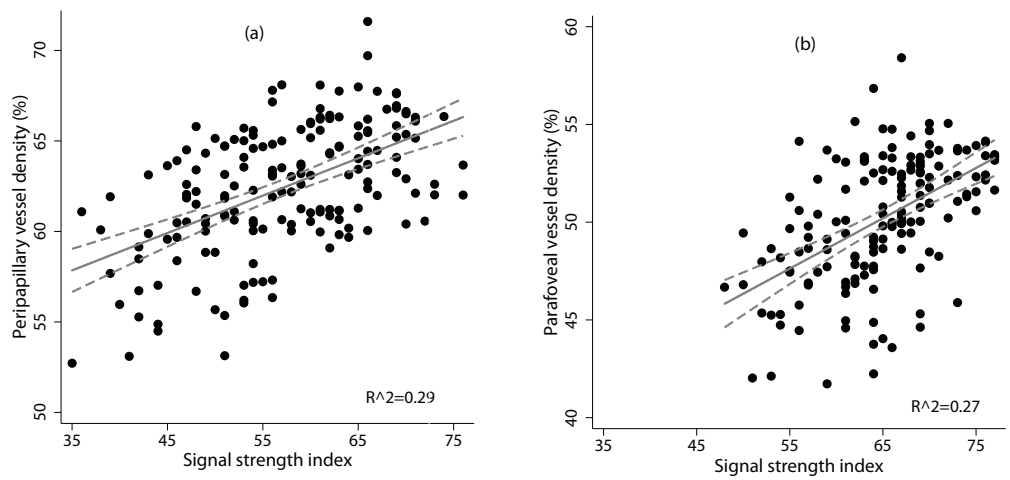

Figure 2. Relationship between signal strength index and the average vessel density in the peripapillary (a) and parafoveal (b) regions.

As the number of subjects with hypertension and diabetes was small, we ran the entire analysis excluding these subjects. The results were similar to the main analysis. SSI still showed a statistically significant association with the vessel densities of all regions (coefficients: 0.12 to 0.26 for peripapillary and 0.23 to 0.30 for macular sectors). Most of the peripapillary vessel densities were still significantly greater in females. 
We also ran the entire analysis considering one eye per subject and the results were similar.

\section{DISCUSSION}

In this study, we evaluated the determinants of peripapillary and macular vessel density parameters of OCTA in normal subjects. Most of the peripapillary vessel densities were higher in females compared to males. Most of the peripapillary vessel densities were lower, while the macular densities were higher, in subjects with systemic hypertension. Vessel densities in all the regions were lower in subjects with diabetes although not statistically significant. Vessel densities in all the regions were significantly greater in scans with higher SSI. Age and optic disc size did not influence the vessel densities of any of the regions. To the best of our knowledge, this is the first study to comprehensively evaluate the effect of multiple determinants on the peripapillary and superficial macular vessel density measurements of OCTA in normal subjects.

A few recent studies have evaluated the effect of determinants on the vessel density measurements of OCTA. However, these were generally restricted to evaluating either the effect of age, gender or refractive error on the vessel density measurements. ${ }^{14-16}$ Yu et al evaluated the effect of age and gender on the macular perfusion parameters of OCTA in healthy Chinese subjects and found that the parafoveal vessel density decreased by $0.4 \%$ per year. We also found a decrease in parafoveal vessel density with age (coefficient: -0.01), but the decrease was $0.2 \%$ per year and was not statistically significant. Another study also found no effect of age on the parafoveal vessel density measurements. ${ }^{16}$ Age also did not influence the peripapillary vessel densities in our study. Yu et al also found that the parafoveal vessel densities were alike in males and females, which is similar to our observation. However, unlike the parafoveal densities, we found that the peripapillary vessel densities were greater in females compared to males. We analyzed the differences between males and females to explain this finding. We found that the females were, on an average, 5 years younger than males. Apart 
from this difference in age, there were no other differences between males and females. Presence of hypertension and diabetes was comparable between males and females as was the SSI of scans, disc area and MRSE. The reason for the peripapillary vessel densities being greater in females, therefore, is unclear and requires validation in future studies.

A study by Wang et al. evaluated the effect of myopia on the peripapillary and parafoveal OCTA parameters and found a significant reduction of vessel density in the peripapillary but not the parafoveal region of high myopia eyes (MRSE of over -6 D). ${ }^{15}$ In our study, though MRSE showed a positive association with peripapillary vessel densities, this association was not statistically significant. Vessel densities in the parafoveal regions were also not affected by MRSE. Our study, unlike the study by Wang et al. however did not include high myopic eyes.

The method of vessel density calculation was different in our study compared to that used in the previous studies. ${ }^{14-16}$ We used the vessel densities provided automatically by the software while the previous studies used either a set threshold decorrelation value ${ }^{14,15}$ or local fractal analysis ${ }^{16}$ to calculate the vessel densities. The median vessel density in the parafoveal region in our study was $50.4 \%$, while the mean was $89.1 \%$ in the study by $Y u$ et $\mathrm{al}^{14}$ and $26.6 \%$ in the study by Wang et al. ${ }^{15}$ The mean superficial parafoveal vessel density using local fractal analysis was $50 \%,{ }^{16}$ very similar to that seen in the present study. The median vessel density in the peripapillary region in our study was $62.7 \%$, while the mean vessel density of the emmetropic eyes in the study by Wang et al was $89.0 \% .{ }^{15}$ Therefore, the method of vessel density estimation should be considered while analysing the differences in the vessel densities reported in all these studies.

Vessel densities in most of the regions were lower in subjects with diabetes. This however was statistically significant only in temporal parafoveal sector. The decrease in vessel densities in diabetics is probably due to the early capillary dropouts that occur prior to clinically identifiable diabetic retinopathy. Previous studies with different imaging modalities have demonstrated the subclinical capillary dropouts in the perifoveal intercapillary area. ${ }^{20-23}$ The decrease in the 
vessel density in the macular region could also be due to the increase in the foveal avascular zone size seen in diabetics. ${ }^{23-25}$

Most of the peripapillary vessel densities were lower, while the macular densities were higher, in subjects with hypertension. It is well known that hypertension leads to arteriolar narrowing 26,27 and that the arteriolar and venular narrowing can precede the development of systemic hypertension. ${ }^{28,} 29$ This might explain the decrease in vessel densities seen in the peripapillary regions in hypertensive individuals. However, the reason for increased vessel density in the macular area is unclear. It should be noted that the number of subjects with hypertension and diabetes was small and future studies with larger sample size should evaluate the relationship between vessel densities and systemic diseases.

SSI of the scans had a significant effect on the vessel densities of all the regions, with the densities being significantly greater in scans with higher SSI. There is no consensus on what value constitutes an adequate SSI. Different studies have used different SSI values, ranging from 30 to 50 , as cut-offs for good quality scans. ${ }^{9}, 11,12$ We analyzed our data considering 50 as the cut-off for the SSI value (137 optic disc and 156 macular scans) and found that the results were similar. SSI still showed a statistically significant association with the vessel densities of all regions (coefficients: 0.09 to 0.26 for peripapillary and 0.25 to 0.32 for macular sectors). Most of the peripapillary vessel densities were still significantly greater in females. Reports on the effect of signal strength on the vessel density measurements of OCTA are sparse. Jia et al in their early work with OCTA reported that the decorrelation values of OCTA were unaffected by signal strength. ${ }^{6}$ However, in contrast, we found that of all the relationships evaluated, the one between the SSI and vessel densities was the most consistent in all the regions. It is possible that the software doesn't differentiate between the static structures and blood vessels efficiently at low SSI scores. Clinicians evaluating the OCTA scans quantitatively therefore should consider the SSI value of the scan during interpretation.

There are a few limitations of the study which should be considered. The vessel density measurements evaluated in this study were the ones automatically 
provided by the software. The software in its current form, does not differentiate between capillaries and large vessels. The effects of the determinant variables may be different on vessels of varying caliber. Further advances in the technology may help to evaluate the capillaries and large vessels separately. OCTA technology depends on the movement of the blood column to detect the vessels. It will therefore not record the presence of a vessel if there is no movement of the blood column or the movement is very slow. Another possible limitation of the current study was that we did not measure the blood pressure of the subjects or record their anti-hypertensive or anti-diabetic medications. Although a previous study has shown no relationship between blood pressure readings and peripapillary vessel densities, ${ }^{9}$ future studies are needed to evaluate the effect of systemic diseases and the medications on the OCTA measurements in greater detail. Future studies should also evaluate the relationship between the vessel densities and the duration of hypertension.

In conclusion, we found that most of the peripapillary vessel densities were higher in females compared to males. Hypertension had a varied effect on vessel densities of different regions. Vessel densities in all the regions were lower in diabetics. Vessel densities in all the regions were significantly greater in scans with higher SSI. These determinants of vessel density should be considered while interpreting the OCTA scans in retinal and ONH diseases.

\section{REFERENCES}

1. Jia Y, Tan O, Tokayer J, et al. Split-spectrum amplitude-decorrelation angiography with optical coherence tomography. Opt Express 2012;20:4710-25.

2. Ishibazawa A, Nagaoka T, Takahashi A, et al. Optical Coherence Tomography Angiography in Diabetic Retinopathy: A Prospective Pilot Study. Am J Ophthalmol 2015;160:35-44.

3. Jia Y, Bailey ST, Wilson DJ, et al. Quantitative optical coherence tomography angiography of choroidal neovascularization in age-related macular degeneration. Ophthalmology 2014;121:1435-44.

4. Kuehlewein L, Bansal M, Lenis TL, et al. Optical Coherence Tomography Angiography of Type 1 Neovascularization in Age-Related Macular Degeneration. Am J Ophthalmol 2015;160:73948.

5. Coscas F, Glacet-Bernard A, Miere A, et al. Optical Coherence Tomography Angiography in 
Retinal Vein Occlusion: Evaluation of Superficial and Deep Capillary Plexa. Am J Ophthalmol 2016;161:160-71.

6. Jia Y, Morrison JC, Tokayer J, et al. Quantitative OCT angiography of optic nerve head blood flow. Biomed Opt Express 2012;3:3127-37.

7. Jia $Y$, Wei $E$, Wang $X$, et al. Optical coherence tomography angiography of optic disc perfusion in glaucoma. Ophthalmology 2014;121:1322-32.

8. Wang $\mathrm{X}$, Jiang $\mathrm{C}$, Ko $\mathrm{T}$, et al. Correlation between optic disc perfusion and glaucomatous severity in patients with open-angle glaucoma: an optical coherence tomography angiography study. Graefes Arch Clin Exp Ophthalmol 2015;253:1557-64.

9. Liu L, Jia Y, Takusagawa HL, et al. Optical Coherence Tomography Angiography of the Peripapillary Retina in Glaucoma. JAMA Ophthalmol 2015;133:1045-52.

10. Leveque PM, Zeboulon P, Brasnu E, et al. Optic Disc Vascularization in Glaucoma: Value of Spectral-Domain Optical Coherence Tomography Angiography. J Ophthalmol 2016;2016:6956717.

11. Yarmohammadi A, Zangwill LM, Diniz-Filho A, et al. Optical Coherence Tomography Angiography Vessel Density in Healthy, Glaucoma Suspect, and Glaucoma Eyes. Invest Ophthalmol Vis Sci 2016;57:OCT451-9.

12. Rao HL, Pradhan ZS, Weinreb RN, et al. Regional Comparisons of Optical Coherence Tomography Angiography Vessel Density in Primary Open-Angle Glaucoma. Am J Ophthalmol 2016;171:75-83.

13. Wei $E$, Jia $Y$, Tan $O$, et al. Parafoveal retinal vascular response to pattern visual stimulation assessed with OCT angiography. PLoS One 2013;8:e81343.

14. Yu J, Jiang C, Wang X, et al. Macular perfusion in healthy Chinese: an optical coherence tomography angiogram study. Invest Ophthalmol Vis Sci 2015;56:3212-7.

15. Wang $X$, Kong $X$, Jiang $C$, et al. Is the peripapillary retinal perfusion related to myopia in healthy eyes? A prospective comparative study. BMJ Open 2016;6:e010791.

16. Gadde SG, Anegondi N, Bhanushali D, et al. Quantification of Vessel Density in Retinal Optical Coherence Tomography Angiography Images Using Local Fractal Dimension. Invest Ophthalmol Vis Sci 2016;57:246-52.

17. Kraus MF, Potsaid B, Mayer MA, et al. Motion correction in optical coherence tomography volumes on a per A-scan basis using orthogonal scan patterns. Biomed Opt Express 2012;3:1182-99.

18. Garway-Heath DF, Poinoosawmy D, Fitzke FW, Hitchings RA. Mapping the visual field to the optic disc in normal tension glaucoma eyes. Ophthalmology 2000;107:1809-15.

19. West BT, Welch KB, Galecki AT. Linear mixed models: a practical guide using statistical software. Boca Raton: CRC Press;2007.

20. Arend $\mathrm{O}$, Wolf $\mathrm{S}$, Jung $\mathrm{F}$, et al. Retinal microcirculation in patients with diabetes mellitus: dynamic and morphological analysis of perifoveal capillary network. Br J Ophthalmol 1991;75:514-8.

21. Arend O, Wolf S, Remky A, et al. Perifoveal microcirculation with non-insulin-dependent diabetes mellitus. Graefes Arch Clin Exp Ophthalmol 1994;232:225-31. 
22. Tam J, Dhamdhere KP, Tiruveedhula $\mathrm{P}$, et al. Disruption of the retinal parafoveal capillary network in type 2 diabetes before the onset of diabetic retinopathy. Invest Ophthalmol Vis Sci 2011;52:9257-66.

23. Salz DA, de Carlo TE, Adhi M, et al. Select Features of Diabetic Retinopathy on Swept-Source Optical Coherence Tomographic Angiography Compared With Fluorescein Angiography and Normal Eyes. JAMA Ophthalmol 2016;134:644-50.

24. Takase N, Nozaki M, Kato A, et al. Enlargement of Foveal Avascular Zone in Diabetic Eyes Evaluated by En Face Optical Coherence Tomography Angiography. Retina 2015;35:237783.

25. Di G, Weihong Y, Xiao Z, et al. A morphological study of the foveal avascular zone in patients with diabetes mellitus using optical coherence tomography angiography. Graefes Arch Clin Exp Ophthalmol 2016;254:873-9.

26. Wong TY, Klein R, Klein BE, et al. Retinal vessel diameters and their associations with age and blood pressure. Invest Ophthalmol Vis Sci 2003;44:4644-50.

27. Koch E, Rosenbaum D, Brolly A, et al. Morphometric analysis of small arteries in the human retina using adaptive optics imaging: relationship with blood pressure and focal vascular changes. J Hypertens 2014;32:890-8.

28. Wong TY, Klein R, Sharrett AR, et al. Retinal arteriolar diameter and risk for hypertension. Ann Intern Med 2004;140:248-55.

29. Ikram MK, Witteman JC, Vingerling JR, et al. Retinal vessel diameters and risk of hypertension: the Rotterdam Study. Hypertension 2006;47:189-94. 


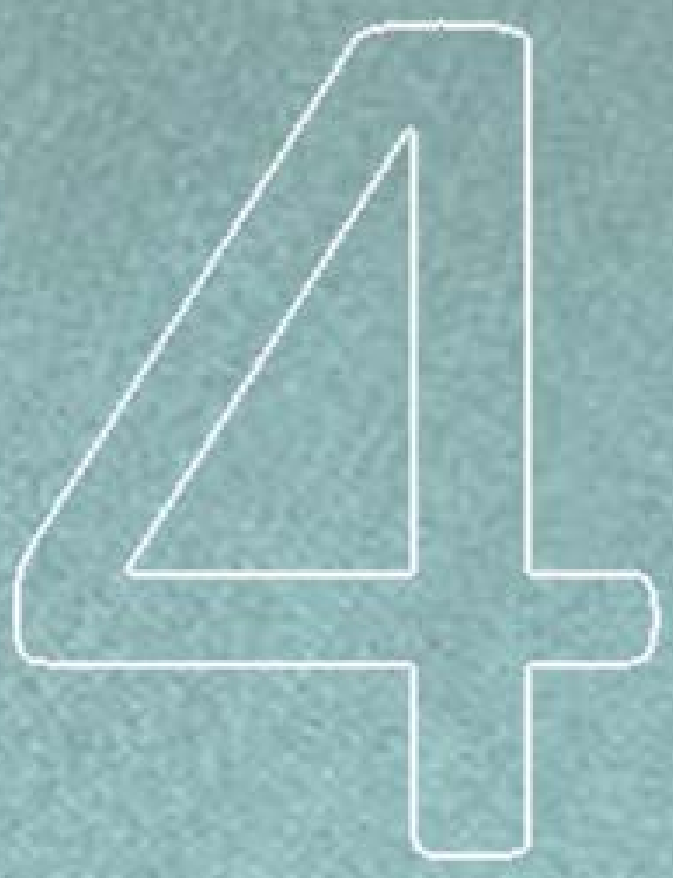

\section{Diagnostic ability of OCT angiography in glaucoma}

Regional comparisons of optical coherence tomography angiography vessel density in primary open angle glaucoma

Harsha L Rao, Zia S Pradhan, Robert N Weinreb, Hemanth B Reddy, Mohammed Riyazuddin, Srilakshmi Dasari, Meena Palakurthy, Narendra K Puttaiah, Dhanaraj A S Rao, Carroll AB Webers.

Am J Ophthalmol 2016 Nov;171:75-83 


\section{ABSTRACT}

Purpose: To compare the diagnostic abilities of the vessel densities in optic nerve head $(\mathrm{ONH})$, peripapillary and macular regions measured using optical coherence tomography angiography (OCTA) in eyes with primary open angle glaucoma (POAG), and to evaluate the effect of glaucoma severity (based on the mean deviation, MD), optic disc size and pre-treatment intraocular pressure (IOP).

Design: Cross-sectional study

Methods: Seventy-eight eyes of 53 control subjects and 64 eyes of 39 POAG patients underwent OCTA imaging. Area under receiver operating characteristic (ROC) curves (AUC) and sensitivities at fixed specificities of vessel densities in $\mathrm{ONH}$, peripapillary and macular regions were analyzed. ROC regression was used to evaluate the effect of covariates on the diagnostic abilities.

Results: The AUCS of ONH vessel densities ranged between 0.59 (superonasal sector) and 0.73 (average inside disc), peripapillary between 0.70 (nasal, superonasal and temporal) and 0.89 (inferotemporal), and macular between 0.56 (nasal) and 0.64 (temporal). AUC of the average peripapillary vessel density was significantly better than the average inside disc $(p=0.05)$ and macular $(p=0.005)$ measurement. MD showed a negative association with the AUCs of the vessel densities of all regions. Pre-treatment IOP (coefficient: 0.09) showed a significant $(p<0.05)$ effect on the AUC of ONH vessel density.

Conclusions: Diagnostic ability of the vessel density parameters of OCTA was only moderate. Macular and inside disc densities had significantly lower diagnostic abilities in POAG than the peripapillary density. Diagnostic abilities of vessel densities increased with increasing severity of glaucoma and that of $\mathrm{ONH}$ vessel density with higher pre-treatment IOPs. 


\section{INTRODUCTION}

Primary open angle glaucoma (POAG) is a chronic progressive optic neuropathy resulting from the apoptosis of the retinal ganglion cells (RGC). ${ }^{1}$ Although the pathogenic mechanisms leading to retinal ganglion cell death are not fully known, intraocular pressure (IOP) induced mechanical injury is a major causal factor as evidenced by the increase in the risk of incident glaucoma and its progression with higher 1 IOP. $^{2}$ It has also been proposed that reduced optic nerve head $(\mathrm{ONH})$ perfusion also plays a role in the pathogenesis of glaucoma. ${ }^{3}, 4$ While some believe that the reduced blood flow seen in glaucoma is secondary to RGC death and thereby a reduced need for perfusion, 5,6 others are of the opinion that the reduced blood flow is the primary event that subsequently leads to the characteristic structural and functional changes of glaucoma. ${ }^{7}$ Earlier studies have measured $\mathrm{ONH}$ blood flow using a variety of techniques and have shown reduction in $\mathrm{ONH}$ perfusion in patients with glaucoma. However, each of these techniques has limitations. ${ }^{8}$

Optical coherence tomography (OCT) has been used to develop a new, threedimensional angiography algorithm called split spectrum amplitude-decorrelation angiography (SSADA) for imaging the retinal and ONH microcirculation. ${ }^{9}$ Early studies have shown that the blood flow measurements provided by OCT angiography (OCTA) are repeatable and reproducible. ${ }^{10-14}$ Studies with OCTA have demonstrated reduced $\mathrm{ONH}$ and peripapillary perfusion in patients with glaucoma. ${ }^{10-13,15}$ OCTA also evaluates the vascular perfusion in the macular region. However, there have been no reports on the macular perfusion in patients with glaucoma. There have not been comparisons to date of the diagnostic abilities of vessel densities in the $\mathrm{ONH}$, peripapillary and macular regions in glaucoma. The purpose of this study was to compare the diagnostic abilities of the vessel density measurements of the $\mathrm{ONH}$, peripapillary and macular regions on OCTA in eyes with POAG. The secondary objective was to evaluate the effect of glaucoma severity, optic disc size and the pre-treatment IOP on the diagnostic abilities of vessel densities. 


\section{METHODS}

This was a cross-sectional study conducted at Narayana Nethralaya, a tertiary eye care center in Bengaluru, South India between September 2015 and March 2016. The methodology adhered to the tenets of the Declaration of Helsinki for research involving human subjects. Written informed consent was obtained from all participants and the study was approved by the Institute's Ethics Committee.

Participants of the study included control subjects and POAG patients. Control subjects were either hospital staff or subjects who consulted for a routine eye examination or a refractive error. Control subjects had no family history of glaucoma, IOP $\leq 21 \mathrm{~mm} \mathrm{Hg}$, normal anterior and posterior segment on clinical examination by an ophthalmologist and non-glaucomatous optic discs, as assessed by experts on masked examination of stereoscopic optic disc photographs. POAG patients had open angles on gonioscopy and glaucomatous changes on optic nerve head examination (neuroretinal rim narrowing, notching and retinal nerve fiber layer defects) as documented by glaucoma experts on dilated examination and confirmed by experts on stereoscopic optic disc photographs. Inclusion criteria for all participants were age $\geq 18$ years, corrected distance visual acuity of $20 / 40$ or better and refractive error within $\pm 5 \mathrm{D}$ sphere and $\pm 3 \mathrm{D}$ cylinder. Exclusion criteria were presence of any media opacities that prevented good quality OCT scans, or any retinal or neurological disease other than glaucoma, which could confound the evaluation. Eyes with a history of trauma or inflammation were also excluded. All participants underwent a comprehensive ocular examination, which included a detailed medical history, corrected distance visual acuity measurement, slit-lamp biomicroscopy, Goldmann applanation tonometry, gonioscopy, dilated fundus examination, visual field (VF) examination and OCTA imaging with RTVue-XR SDOCT (Optovue Inc., Fremont, CA). Baseline (pre-treatment) IOP, the IOP at which anti-glaucoma treatment was started, was documented for all POAG eyes.

Stereoscopic optic disc photographs were obtained by trained technicians using a digital fundus camera (Kowa nonmyd WX, Kowa Company, Ltd., Japan). Each optic disc photograph was evaluated independently by two glaucoma experts 
(HLR and NKP) in a masked manner to determine the presence of glaucomatous changes (focal or diffuse neuroretinal rim thinning, localized notching or RNFL defects). The experts were masked to all the clinical data, visual field data and the other eye data. Discrepancy in the classification between the two experts was adjudicated by a third glaucoma expert (ZSP).

VF examination was performed using a Humphrey Field analyzer II, model 720i (Zeiss Humphrey Systems, Dublin, CA), with the Swedish interactive threshold algorithm (SITA) standard 24-2 program. VFs were considered reliable if the fixation losses were less than $20 \%$, and the false positive and false negative response rates were less than $15 \%$. VF result was not considered for the definition of glaucoma or the controls but was used for the grading of glaucoma severity.

OCTA imaging of the optic disc, peripapillary region and macula was performed using RTVue-XR SD-OCT (AngioVue, v2015.100.0.33). RTVue-XR uses an 840 $\mathrm{nm}$ diode laser source, with an A-scan rate of $70 \mathrm{kHz}$ per second. Imaging is performed using a set of 2 scans; one vertical priority and one horizontal priority raster volumetric scan. The optic disc scan covers an area of $4.5 \times 4.5 \mathrm{~mm}$ and the macular scan was performed using volumetric scans covering $3 \times 3 \mathrm{~mm}$. An orthogonal registration algorithm is used to produce merged 3-dimensional OCT angiograms. ${ }^{16}$ The SSADA algorithm compares the consecutive B-scans at the same location to detect flow using motion contrast. ${ }^{9}$ Vessel densities are calculated over the entire scan area, i.e. whole enface disc and whole enface macula. Vessel density is defined as the percentage area occupied by the large vessels and microvasculature in a particular region. In addition to the whole scan analysis, the software calculates vessel densities in various layers of the retina and the $\mathrm{ONH}$, and each scan region is further divided into sectors as described below. An en face angiogram of the $\mathrm{ONH}$ and peripapillary circulation is obtained by the maximum flow (decorrelation value) projection from the inner limiting membrane (ILM) to retinal pigment epithelium (RPE). The $\mathrm{ONH}$ vessel densities were calculated from the "nerve head" segment of the ONH angiogram (Figure 1a). This segment extends from 2000 microns above the internal limiting membrane (ILM) to 150 microns below the ILM. The software automatically fits an ellipse to the optic disc margin and calculates the average vessel density within the $\mathrm{ONH}$ (referred 
to as the inside disc vessel density). It also divides the $\mathrm{ONH}$ into 6 sectors based on the Garway-Heath map and calculates the vessel densities in each ONH sector (nasal, inferonasal, inferotemporal, superotemporal, superonasal and temporal sectors) as represented in Figure $1 a .{ }^{17}$ The peripapillary region is defined as a $0.75 \mathrm{~mm}$-wide elliptical annulus extending from the optic disc boundary (Figure $1 b)$. The peripapillary vessels were analyzed in superficial retinal layers from the Radial Peripapillary Capillary (RPC) segment. RPC segment extends from the ILM to the nerve fiber layer. The peripapillary region was also divided into 6 sectors based on the Garway-Heath map (Figure $1 \mathrm{~b}$ ) and vessel densities for the entire peripapillary area (average) and each sector were determined. ${ }^{17}$ Macular vessel densities analyzed in this study was of the superficial vascular plexus present in the inner layers of the retina (extending from the internal limiting membrane to the inner plexiform layer). Macular vessel densities were analyzed over a 1.5 $\mathrm{mm}$-wide parafoveal, circular annulus centered on the macula (Figure 1c). The parafoveal region was also divided into 4 sectors of $90^{\circ}$ each (nasal, inferior, superior and temporal sectors). Image quality was assessed for all OCTA scans. Poor quality images with a signal strength index (SSI) less than 35 or images with residual motion artifacts were excluded from the analysis.

(a)

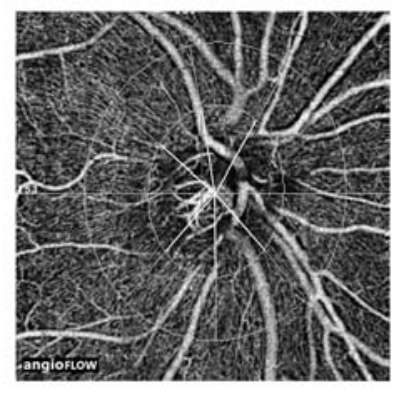

(b)

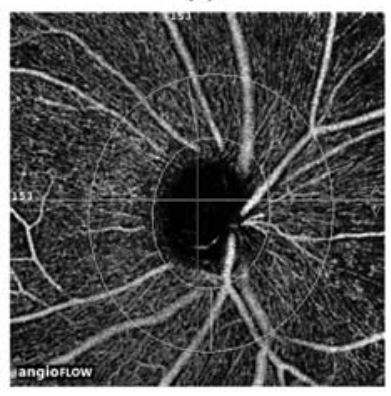

(c)

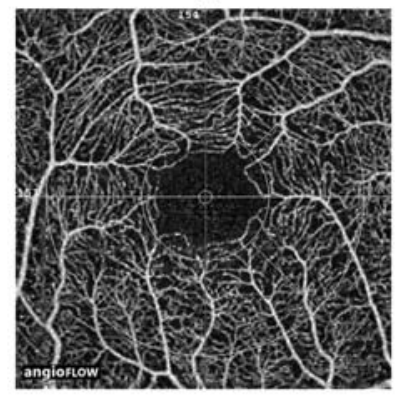

Figure 1. Figure showing the (a) optic nerve head (b) peripapillary and (c) macular optical coherence tomography angiography images and the sectors where vessel densities are calculated. The optic disc vessel density is calculated within the optic nerve head from the nerve head segment of the en face angiogram (a), peripapillary vessel density over a $0.75 \mathrm{~mm}$-wide elliptical annulus extending from the optic disc boundary from the radial peripapillary capillary segment (b), and superficial macular vessel density over a $1.5 \mathrm{~mm}$-wide circular annulus centered on the macula (c). 
All subjects also underwent optic disc area measurement on RTVue-XR SD-OCT using the traditional ONH scan. This scan consists of 12 radial scans $3.4 \mathrm{~mm}$ in length and 6 concentric ring scans ranging from 2.5 to $4.0 \mathrm{~mm}$ in diameter all centered on the optic disc. Retinal pigment epithelium (RPE) tips are automatically detected by the software and the RPE tips are joined to delineate the optic disc margin and to calculate the disc area. All the examinations for a particular subject were performed on the same day.

\section{STATISTICAL ANALYSIS}

Descriptive statistics included mean and standard deviation for normally distributed variables and median and inter-quartile range (IQR) for non-normally distributed variables. Shapiro-Wilk test was used to test for the normality distribution of continuous variables. Receiver operating characteristic (ROC) curves were used to describe the ability of OCTA vessel densities to discriminate glaucomatous eyes from control eyes. Sensitivities at fixed specificities of $80 \%$ and $95 \%$ were determined for all the parameters. To obtain confidence intervals for area under the ROC curves (AUC) and sensitivities, a bootstrap re-sampling procedure was used ( $n=1000$ re-samples). As measurements from both eyes of the same subject are likely to be correlated, the standard statistical methods for parameter estimation lead to underestimation of standard errors and to confidence intervals that are too narrow. ${ }^{18}$ Therefore, the cluster of data for the study subject was considered as the units of resampling and bias corrected standard errors were calculated during all estimations. This procedure has been used to adjust for the presence of multiple correlated measurements from the same unit. ${ }^{19,} 20$ ROC regression modeling technique was used to evaluate the effect of glaucoma severity, disc size and the pre-treatment IOP on the AUCs and sensitivities of OCTA parameters in diagnosing glaucoma. ${ }^{21,22}$

Statistical analyses were performed using commercial software (Stata ver. 13.1; StataCorp, College Station, TX). A p value of $\leq 0.05$ was considered statistically significant. 


\section{RESULTS}

One hundred and seventy-one eyes of 102 subjects ( 86 eyes of 56 subjects diagnosed as normal and 85 eyes of 46 subjects diagnosed as POAG on clinical examination) underwent OCTA imaging with SD-OCT. Among these, 19 eyes of 13 patients in which the optic disc classification on stereo photographs was not glaucomatous optic neuropathy, were excluded. Of the remaining eyes, 10 eyes with poor disc scans, 15 eyes with poor macular scans and 10 eyes both poor disc and macular scans were excluded. Final analysis included 132 optic disc scans and 127 macular scans obtained from 142 eyes of 92 subjects (78 eyes of 53 normal and 64 eyes of 39 POAG subjects). Of the 64 eyes with POAG, 12 eyes (11 patients) had a "within normal limit" or a "borderline" glaucoma hemifield test result, or the probability value of pattern standard deviation $>5 \%$ or both on VF (preperimetric glaucoma). Half the number of eyes included in the POAG group had a mean deviation of better than $-5 \mathrm{~dB}$ on VF. Of the 64 eyes with POAG, 13 eyes were on topical beta blockers, 10 on alpha agonists, 13 on carbonic anhydrase inhibitors and 41 on prostaglandin analogues (either as a monotherapy or as components of the combination therapy). Table 1 shows the clinical, VF and vessel density measurements of all subjects. POAG patients were significantly older than control subjects. SSI of the optic disc scans was significantly greater in the control as compared to the POAG patients. AUCs and sensitivities at fixed specificities of optic disc and peripapillary vessel density parameters were therefore calculated after adjusting for the difference in age and signal strength between the control and POAG groups using covariate-adjustment as proposed by Pepe. ${ }^{23}$ AUCs and sensitivities at fixed specificities of macular vessel density parameters were similarly calculated after adjusting for the difference in age. All the $\mathrm{ONH}$, peripapillary and macular vessel densities were significantly lesser in the glaucoma compared to the control group. The magnitude of difference in the median vessel densities between the glaucoma and the control groups was significantly smaller in the macular region compared to the $\mathrm{ONH}$ and peripapillary regions. 
Table 1. Clinical features, visual field parameters and vessel density measurements of the participants. All values represent median and interquartile range unless specified.

\begin{tabular}{|c|c|c|c|}
\hline & $\begin{array}{c}\text { Control group } \\
\text { (78 eyes, } 53 \text { subjects) }\end{array}$ & $\begin{array}{c}\text { POAG group } \\
\text { (64 eyes, } 39 \text { patients) }\end{array}$ & $\mathbf{P}$ \\
\hline Age (years) & $58(52,65)$ & $66(57,72)$ & 0.01 \\
\hline Gender (male:female) & $29: 24$ & 28:11 & 0.10 \\
\hline Sphere (D) & $0.5(0,1)$ & $0(-0.75,0.5)$ & 0.02 \\
\hline Cylinder (D) & $-0.5(-1,-0.5)$ & $-0.75(-1,-0.25)$ & 0.67 \\
\hline Optic disc area $\left(\mathrm{mm}^{2}\right)$ & $2.30(2.00,2.58)$ & $2.33(1.98,2.59)$ & 0.93 \\
\hline Pre-treatment IOP (mm Hg) & $16(14,18)$ & $19(16,24)$ & $<0.001$ \\
\hline Hypertension (yes:no) & $16: 37$ & $16: 23$ & 0.28 \\
\hline Diabetes mellitus (yes:no) & $15: 38$ & 9:30 & 0.57 \\
\hline Mean deviation $(\mathrm{dB})$ & $-1.1(-3.0,-0.2)$ & $-5.3(-9.6,-3.1)$ & $<0.001$ \\
\hline Pattern standard deviation $(\mathrm{dB})$ & $1.7(1.5,2.5)$ & $4.7(2.8,9.2)$ & $<0.001$ \\
\hline Visual field index (\%) & $99(98,99)$ & $90(75,95)$ & $<0.001$ \\
\hline SSI (Optic disc scan)* & $54.2 \pm 9.5$ & $50.4 \pm 8.2$ & 0.02 \\
\hline Whole enface vessel density (disc scan) & $54.4(51.7,56.9)$ & $48.0(42.9,53.4)$ & $<0.001$ \\
\hline Inside disc vessel density & $47.4(43.0,50.4)$ & $40.2(34.8,47.3)$ & $<0.001$ \\
\hline Nasal vessel density (\%) & $48.8(42.9,52.5)$ & $41.6(35.8,47.6)$ & $<0.001$ \\
\hline Inferonasal vessel density (\%) & $51.9(44.8,56.7)$ & $47.5(39.9,53.8)$ & 0.008 \\
\hline Inferotemporal vessel density (\%) & $46.2(41.1,52.3)$ & $39.7(33.4,47.5)$ & $<0.001$ \\
\hline Superotemporal vessel density (\%) & $47.6(41.9,52.0)$ & $39.5(28.3,47.2)$ & $<0.001$ \\
\hline Superonasal vessel density (\%) & $50.1(42.9,55.2)$ & $43.7(37.2,52.1)$ & 0.004 \\
\hline Temporal vessel density (\%) & $44.5(39.1,52.3)$ & $36.2(28.7,45.4)$ & $<0.001$ \\
\hline Average Peripapillary vessel density (\%) & $62.0(60.0,64.4)$ & $55.5(50.8,59.5)$ & $<0.001$ \\
\hline Nasal vessel density (\%) & $59.7(57.2,62.1)$ & $56.0(48.7,59.6)$ & $<0.001$ \\
\hline Inferonasal vessel density (\%) & $63.4(60.7,66.5)$ & $55.2(46.2,60.6)$ & $<0.001$ \\
\hline Inferotemporal vessel density (\%) & $66.0(63.5,68.4)$ & $55.5(44.5,59.9)$ & $<0.001$ \\
\hline Superotemporal vessel density (\%) & $66.6(64.0,68.8)$ & $59.4(53.4,65.4)$ & $<0.001$ \\
\hline Superonasal vessel density (\%) & $62.9(56.5,66.1)$ & $55.3(48.7,61.2)$ & $<0.001$ \\
\hline Temporal vessel density (\%) & $60.4(57.9,64.2)$ & $57.0(50.6,60.1)$ & $<0.001$ \\
\hline SSI (Macula scan)* & $62.0 \pm 6.9$ & $60.6 \pm 7.7$ & 0.28 \\
\hline Whole enface vessel density (macula scan) & $48.3(45.9,50.3)$ & $44.7(41.8,47.6)$ & $<0.001$ \\
\hline Foveal vessel density (\%) & $25.5(22.9,27.7)$ & $24.9(22.3,27.8)$ & 0.58 \\
\hline Parafoveal vessel density (\%) & $49.8(47.7,52.7)$ & $47.5(44.7,50.2)$ & $<0.001$ \\
\hline Nasal vessel density (\%) & $48.5(46.7,51.1)$ & $47.0(44.1,48.9)$ & 0.01 \\
\hline Inferior vessel density (\%) & $51.6(48.1,53.9)$ & $48.9(44.8,51.7)$ & 0.001 \\
\hline Superior vessel density (\%) & $50.8(48.2,53.3)$ & $47.6(45.0,52.0)$ & 0.002 \\
\hline Temporal vessel density (\%) & $49.8(47.5,52.5)$ & $47.3(45.0,50.1)$ & 0.001 \\
\hline
\end{tabular}

D: diopter; dB: decibel; IOP: intraocular pressure; SSI: signal strength index; *mean \pm standard deviation. 
The AUCs and sensitivities at fixed specificities of the vessel density measurements to differentiate POAG from control eyes are shown in Table 2. The AUCs of ONH vessel densities ranged between 0.59 (superonasal sector) and 0.73 (average inside disc density). Sensitivities at 95\% specificity ranged between $11 \%$ (superotemporal sector) and $25 \%$ (inside disc density). The AUCs of peripapillary vessel densities ranged between 0.70 (nasal, superonasal and temporal sector) and 0.89 (inferotemporal sector), and macular vessel densities between 0.56 (nasal sector) and 0.64 (temporal sector). The sensitivities at $95 \%$ specificity of peripapillary vessel densities ranged between $25 \%$ (superonasal sector) and $68 \%$ (inferotemporal sector), and macular vessel densities between $3 \%$ (nasal sector) and $15 \%$ (superior sector). The parameters with the highest diagnostic abilities were the whole enface vessel density of the disc scan (AUC of 0.90) and the inferotemporal sector peripapillary vessel density (AUC of 0.89). Figure 2 shows the ROC curves of the vessel density measurements of the inside disc, average peripapillary and parafoveal regions. AUC of average peripapillary vessel density was significantly better than that of the inside disc $(p=0.05)$ and the parafoveal $(p=0.005)$ measurements. AUC of the inside disc vessel density was comparable to that of the average parafoveal vessel density $(p=0.31)$.

Table 2. Diagnostic ability of vessel density parameters in differentiating open angle glaucoma from control eyes (figures in parenthesis represent $95 \%$ confidence intervals).

\begin{tabular}{lccc}
\hline Vessel density & AUC & $\begin{array}{c}\text { Sensitivity at } \\
95 \% \text { specificity }\end{array}$ & $\begin{array}{c}\text { Sensitivity at 80\% } \\
\text { specificity }\end{array}$ \\
\hline Whole enface (disc scan) & $0.90(0.81-0.95)$ & $67 \%(39-84)$ & $82 \%(62-92)$ \\
Inside disc & $0.73(0.61-0.84)$ & $25 \%(04-52)$ & $57 \%(39-75)$ \\
Nasal & $0.70(0.57-0.80)$ & $22 \%(04-49)$ & $54 \%(27-68)$ \\
Inferonasal & $0.61(0.49-0.72)$ & $13 \%(03-28)$ & $43 \%(24-66)$ \\
Inferotemporal & $0.64(0.53-0.74)$ & $13 \%(03-26)$ & $40 \%(21-56)$ \\
Superotemporal & $0.71(0.60-0.81)$ & $11 \%(02-21)$ & $48 \%(32-67)$ \\
Superonasal & $0.59(0.47-0.70)$ & $16 \%(04-32)$ & $24 \%(08-42)$ \\
Temporal & $0.67(0.53-0.78)$ & $13 \%(02-42)$ & $48 \%(16-67)$ \\
Average Peripapillary & $0.83(0.74-0.90)$ & $47 \%(29-60)$ & $72 \%(49-86)$ \\
$\quad$ Nasal & $0.70(0.56-0.81)$ & $27 \%(13-40)$ & $52 \%(31-77)$ \\
Inferonasal & $0.81(0.73-0.88)$ & $53 \%(37-73)$ & $67 \%(49-81)$ \\
Inferotemporal & $0.89(0.81-0.94)$ & $68 \%(45-81)$ & $78 \%(62-90)$
\end{tabular}




\begin{tabular}{llll} 
Superotemporal & $0.76(0.66-0.84)$ & $33 \%(14-45)$ & $58 \%(41-75)$ \\
Superonasal & $0.70(0.59-0.80)$ & $25 \%(12-41)$ & $50 \%(30-67)$ \\
Temporal & $0.70(0.59-0.80)$ & $28 \%(05-48)$ & $50 \%(35-68)$ \\
Whole enface (macula scan) & $0.69(0.56-0.79)$ & $20 \%(02-51)$ & $54 \%(38-73)$ \\
Parafoveal & $0.63(0.48-0.75)$ & $09 \%(02-28)$ & $34 \%(12-52)$ \\
Nasal & $0.56(0.42-0.69)$ & $03 \%(01-20)$ & $31 \%(09-52)$ \\
Inferior & $0.61(0.48-0.73)$ & $10 \%(02-46)$ & $37 \%(11-52)$ \\
Superior & $0.63(0.50-0.74)$ & $15 \%(05-43)$ & $39 \%(16-63)$ \\
Temporal & $0.64(0.50-0.77)$ & $07 \%(02-26)$ & $39 \%(14-64)$ \\
\hline
\end{tabular}

AUC: area under the receiver operating characteristic curve.

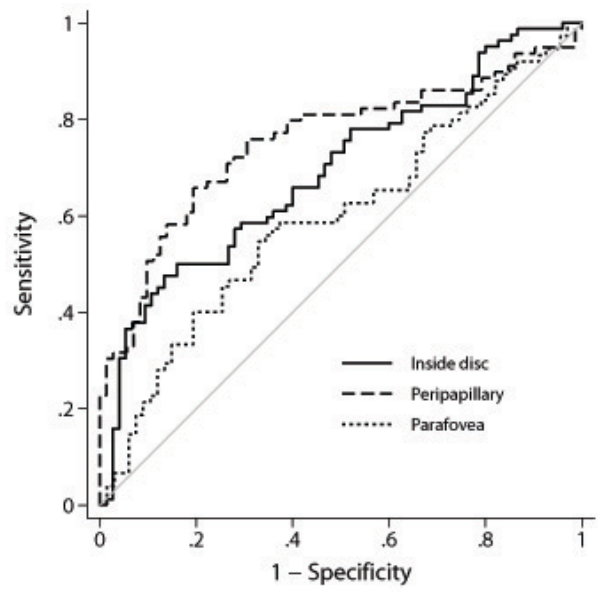

Figure 2. Receiver operating characteristic curves of inside disc, peripapillary and parafoveal vessel density measurements. AUC of parafoveal and inside disc vessel densities were significantly lesser than that of the peripapillary measurements.

Table 3 shows the results of the univariate ROC regression analyses evaluating the effect of disease severity (based on the mean deviation, MD of VF), optic disc area and pre-treatment IOP on the diagnostic abilities of the average vessel density parameters of the three regions. MD had a negative association with the AUCs of all vessel density parameters. AUCs of vessel densities of all regions increased as the MD decreased (glaucoma severity increased). Figure 3a shows the effect of MD on the sensitivities at $95 \%$ specificity of the inside disc, average peripapillary and parafoveal vessel density measurements. Optic disc size had no influence 
on the AUCs of inside disc, peripapillary or the parafoveal measurements. Pretreatment IOP had a significant positive relationship with the AUCs of inside disc vessel density but did not influence the AUCS of peripapillary or the parafoveal measurements. AUC and sensitivities at fixed specificities of inside disc vessel density increased significantly in eyes with higher pre-treatment IOPs. Figure $3 \mathrm{~b}$ shows the effect of pre-treatment IOP on the sensitivities at 95\% specificity of the inside disc, average peripapillary and parafoveal vessel density measurements.

Table 3. Results of the univariate receiver operating characteristic $(\mathrm{ROC})$ regression models evaluating the effect of covariates on the area under the ROC curves of the vessel density measurements inside the optic disc, peripapillary and parafoveal region. Figures represent coefficient with $95 \%$ confidence interval in parenthesis.

\begin{tabular}{lccc}
\hline \multicolumn{1}{c}{ Parameter } & $\begin{array}{c}\text { Inside disc } \\
\text { vessel density }\end{array}$ & $\begin{array}{c}\text { Peripapillary } \\
\text { vessel density }\end{array}$ & $\begin{array}{c}\text { Parafoveal } \\
\text { vessel density }\end{array}$ \\
\hline Mean deviation & $-0.06(-0.13,0.01)$ & $-0.05(-0.14,0.02)$ & $-0.05(-0.13,0.03)$ \\
Disc area & $-0.07(-1.06,0.69)$ & $-0.18(-0.62,0.35)$ & $0.17(-0.46,0.80)$ \\
Pre-treatment IOP & $0.09(0.04,0.18)^{*}$ & $0.01(-0.05,0.08)$ & $0.01(-0.04,0.05)$ \\
\hline
\end{tabular}

* - statistically significant $(p<0.05)$. IOP: intraocular pressure; Models evaluating the area under the ROC curves of inside disc and peripapillary vessel densities were adjusted for the difference in mean age and signal strength index between the control and glaucoma group. Model evaluating the area under the ROC curve of parafoveal vessel density was adjusted for the difference in the age between the control and the glaucoma group.
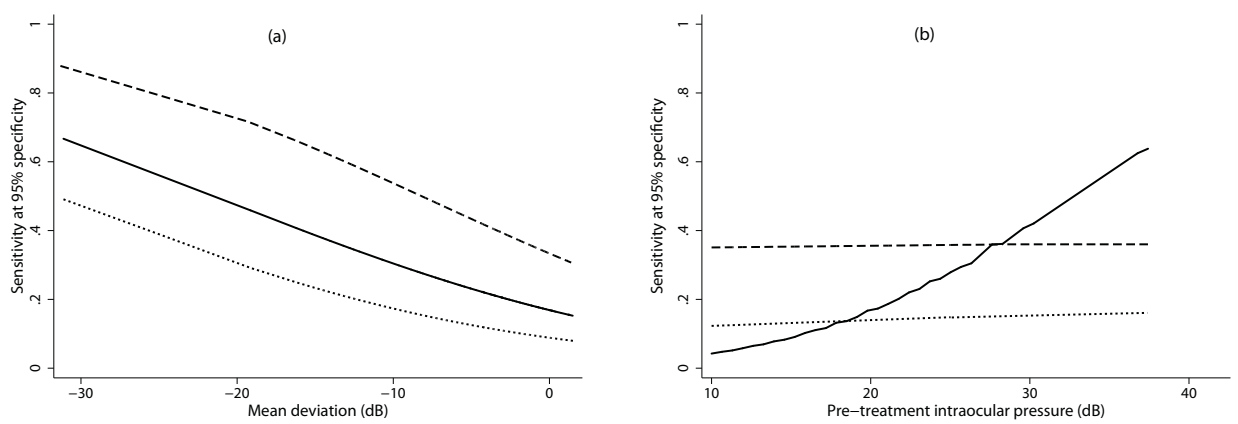

Figure 3. Sensitivity at $95 \%$ specificity of inside disc vessel density (solid line), peripapillary vessel density (dashed line) and parafoveal vessel density (dotted line) according to mean deviation on visual fields (a), and pre-treatment intraocular pressure (b). 


\section{DISCUSSION}

In the current study, a $15 \%$ reduction was found in the median inside disc vessel density in POAG eyes compared to the normal eyes. Previous studies have reported an $\mathrm{ONH}$ vessel density reduction of $10 \%$ to $34 \%$ in glaucomatous eyes. ${ }^{11}$, $12,15 \mathrm{Jia}$ et al also reported that the reduction in $\mathrm{ONH}$ vessel density was greater in the temporal part of the $\mathrm{ONH}(57 \%)$, which is devoid of the major retinal vessels, compared to the entire $\mathrm{ONH}(34 \%) .{ }^{10}$ In contrast to that reported by Jia, et al., the ONH vessel density decrease noted in our study in the temporal sector (19\%) was similar to that of the entire $\mathrm{ONH}$. This difference may be related to the dissimilarity in the definition of the ONH sectors between the 2 studies. Additionally, the ONH (inside disc) vessel densities in our study (which had a considerably larger sample size) showed a significant variability ranging from $27 \%$ to $60 \%$ in normal eyes. The variability was larger when analyzed sectorwise. This variability in $\mathrm{ONH}$ vessel density may be related to the physiological variations generally seen in the $\mathrm{ONH}$ with respect to the disc size, shape, tilt, position of central retinal vessels, etc. We found an $11 \%$ reduction in the average peripapillary vessel density in glaucomatous eyes (median MD: -5 dB). Study on the peripapillary vessel density by Liu et al evaluated 12 glaucomatous eyes (average mean deviation: $-6.05 \mathrm{~dB}$ ) and found a reduction in peripapillary vessel density of $13 \%$ when compared to that in 12 control eyes. ${ }^{13}$ Our results are comparable to that by Liu et al. considering the fact that the severity of glaucoma in our patients was less than that in the study by Liu et al. In fact, 12 of 64 POAG eyes in our study had normal VF and half the number of POAG eyes had a VF MD of better than $-5 \mathrm{~dB}$. Reduction in the median vessel density noted in different peripapillary sectors in our study ranged between $6 \%$ and $16 \%$.

To the best of our knowledge, there have not been any studies reported that have evaluated the vessel densities at the macula in glaucomatous eyes. Previous studies that have quantified the macular vessel densities in normal eyes using OCTA have used different methods. ${ }^{14,24,25}$ A recent study used local fractal analysis to calculate macular vessel densities in normal eyes and reported values similar to that found in the control group of our study. ${ }^{25}$ Sector-wise reduction 
in median vessel densities in the parafoveal region noted in our study ranged between $3 \%$ and $6 \%$. The magnitude of difference in the median vessel density measurements between the glaucoma and the control groups was significantly smaller in the macular region compared to $\mathrm{ONH}$ and peripapillary regions.

On evaluating the ability of vessel densities of the three scanning regions in differentiating glaucoma eyes from control eyes, the macular and inside disc vessel densities had significantly lower diagnostic abilities in POAG compared to the vessel density in the peripapillary regions. To the best of our knowledge, there also have not been any published reports describing the diagnostic ability of macular vessel density measurements in glaucoma. Poor diagnostic ability of the vessel density at the macula, the region with the highest density of RGCs, probably points towards the fact that the vasculature is not the primary target in the pathogenesis of glaucoma and the RGC loss is independent of vascular alterations.

Diagnostic ability of even the best vessel density parameter of the peripapillary region was only moderate, with an AUC of 0.89 and sensitivity at $95 \%$ specificity of $68 \%$. Liu et al evaluated the diagnostic ability of peripapillary vessel density in 12 (9 perimetric and 3 pre-perimetric) glaucoma and 12 normal eyes, and reported an AUC of 0.94 , specificity of $91.7 \%$ and sensitivity of $83.3 \%$. The diagnostic ability estimates of peripapillary vessel density reported in their study were significantly higher than that found in our study. However, the sample size in the study by Liu et al was too small to make meaningful comparisons with our study results.

Diagnostic abilities of vessel densities in all regions increased with increasing severity of glaucoma. However, the $95 \% \mathrm{Cls}$ of the coefficients for the association between MD and the AUC included zero. We might have failed to detect a statistically significant effect of severity on the diagnostic abilities of vessel densities because our glaucoma group consisted predominantly of preperimetric and early disease. Similar effect of disease severity has been reported on the diagnostic ability of ONH vessel density by Wang et al. AUC of ONH vessel density which was 0.80 in the study by Wang et al when the entire glaucoma group was included, increased to 0.90 when only eyes with severe glaucoma ( $M D<-12 \mathrm{~dB}$ ) were considered. ${ }^{12}$ 
Diagnostic ability of $\mathrm{ONH}$ vessel density also increased in eyes with higher pre-treatment IOP. Pre-treatment IOP did not affect the diagnostic ability of peripapillary and macular vessel densities. It is generally hypothesized that the vascular mechanisms contributing to the pathogenesis of glaucoma are not IOP-independent. IOP related stress and strain are hypothesized to occlude the capillaries especially in the lamina cribrosa of the $\mathrm{ONH}^{26}$ The level of IOP therefore was expected to have a positive influence on the diagnostic abilities of vessel densities when accounting for the glaucoma severity. On the contrary, if reduced blood flow was the predominant pathogenic mechanism in POAG eyes with low pre-treatment IOPs (normal tension glaucoma), then we would have seen a negative association between IOP and the diagnostic ability of vessel densities when the glaucoma severity was accounted for. We however noticed a positive influence of IOP on the diagnostic ability of the ONH vessel densities, but not of the peripapillary and macular vessel densities. This suggests that the vessel density decrease in the macular and the peripapillary retinal vessels in glaucoma might be independent of the IOP levels at which the glaucoma develops. Future studies should explore and validate this finding.

There are some limitations of the OCTA technology and the study design which need to be considered while interpreting the results. The vessel density measurements evaluated in this study were the ones automatically provided by the software. The software in its current form does not differentiate the changes in capillaries from that in large vessels. The software also does not provide further insights into the nature of vascular changes such as attenuation, drop-out, etc. The technology also doesn't evaluate the choroidal vasculature. These additional details would provide a better understanding of the vascular changes in glaucoma. Another possible limitation of the current study was that we did not measure the blood pressure of the subjects or record their systemic anti-hypertensive medication. However, we recorded the history of hypertension and found that the number of subjects with hypertension was similar between the glaucoma and the control groups. A previous study also has shown no relationship between blood pressure readings and peripapillary vessel densities on OCTA. ${ }^{13}$ POAG patients were also on different classes of topical anti-glaucoma medications and these medications could have affected the vessel densities. However, there are 
no reports to date, on the effect of topical anti-glaucoma medications on the vessel densities. In the same context, the peripapillary vessel densities can also be affected by parapapillary atrophy (PPA). We did not record the presence of PPA or its extent in our subjects. Future studies should evaluate the determinants of ocular perfusion on OCTA in greater detail. Another limitation of the study is the case-control design, with a clear distinction between glaucoma patients (cases) defined based on the presence of glaucomatous optic nerve head changes, and normal subjects (controls) with no suspicious findings of glaucoma. Such a design has been shown to overestimate the actual diagnostic ability of a test. ${ }^{27-29}$

In conclusion, we found that the diagnostic ability of even the best vessel density parameter (inferotemporal sector measurement of the peripapillary region) was only moderate. Moreover, the macular and inside disc vessel densities had significantly lower diagnostic abilities in POAG compared to the vessel densities in the peripapillary region. Diagnostic abilities of vessel densities of all regions increased with increasing severity of glaucoma. Diagnostic ability of ONH vessel density increased in eyes with higher pre-treatment IOP.

\section{REFERENCES}

1. Weinreb RN, Khaw PT. Primary open-angle glaucoma. Lancet 2004;363:1711-1720.

2. Weinreb RN, Aung T, Medeiros FA. The pathophysiology and treatment of glaucoma: a review. JAMA 2014;311:1901-1911.

3. Bonomi L, Marchini G, Marraffa M, Bernardi P, Morbio R, Varotto A. Vascular risk factors for primary open angle glaucoma: the Egna-Neumarkt Study. Ophthalmology 2000;107:12871293.

4. Leske MC, Heijl A, Hyman L, et al. Predictors of long-term progression in the Early Manifest Glaucoma Trial. Ophthalmology 2007;114:1965-1972.

5. Rojanapongpun P, Drance SM, Morrison BJ. Ophthalmic artery flow velocity in glaucomatous and normal subjects. Br J Ophthalmol 1993;77:25-29.

6. Moore D, Harris A, Wudunn D, Kheradiya N, Siesky B. Dysfunctional regulation of ocular blood flow: A risk factor for glaucoma? Clin Ophthalmol 2008;2:849-861.

7. Harris A, Sergott RC, Spaeth GL, Katz JL, Shoemaker JA, Martin BJ. Color Doppler analysis of ocular vessel blood velocity in normal-tension glaucoma. Am J Ophthalmol 1994;118:642649. 
8. Weinreb RN, Harris A., editor. Ocular Blood Flow in Glaucoma, The 6th Consensus Report of the World Glaucoma Association. Amsterdam: Kugler Publications, 2009:19-56.

9. Jia Y, Tan O, Tokayer J, et al. Split-spectrum amplitude-decorrelation angiography with optical coherence tomography. Opt Express 2012;20:4710-4725.

10. Jia Y, Morrison JC, Tokayer J, et al. Quantitative OCT angiography of optic nerve head blood flow. Biomed Opt Express 2012;3:3127-3137.

11. Jia $Y$, Wei $E$, Wang $X$, et al. Optical coherence tomography angiography of optic disc perfusion in glaucoma. Ophthalmology 2014;121:1322-1332.

12. Wang $\mathrm{X}$, Jiang $\mathrm{C}$, Ko $\mathrm{T}$, et al. Correlation between optic disc perfusion and glaucomatous severity in patients with open-angle glaucoma: an optical coherence tomography angiography study. Graefes Arch Clin Exp Ophthalmol 2015;253:1557-1564.

13. Liu L, Jia Y, Takusagawa HL, et al. Optical Coherence Tomography Angiography of the Peripapillary Retina in Glaucoma. JAMA Ophthalmol 2015;133:1045-1052.

14. Yu J, Jiang $C$, Wang $X$, et al. Macular perfusion in healthy Chinese: an optical coherence tomography angiogram study. Invest Ophthalmol Vis Sci 2015;56:3212-3217.

15. Leveque PM, Zeboulon P, Brasnu E, Baudouin C, Labbe A. Optic Disc Vascularization in Glaucoma: Value of Spectral-Domain Optical Coherence Tomography Angiography. J Ophthalmol 2016;20166956717.

16. Kraus MF, Potsaid B, Mayer MA, et al. Motion correction in optical coherence tomography volumes on a per A-scan basis using orthogonal scan patterns. Biomed Opt Express 2012;3:1182-1199.

17. Garway-Heath DF, Poinoosawmy D, Fitzke FW, Hitchings RA. Mapping the visual field to the optic disc in normal tension glaucoma eyes. Ophthalmology 2000;107:1809-1815.

18. Glynn RJ, Rosner B. Accounting for the correlation between fellow eyes in regression analysis. Arch Ophthalmol 1992;110:381-387.

19. Zhou XH, Obuchowski NA, McClish DK. Analysis of correlated ROC data. In: Zhou XH, Obuchowski NA, McClish DK, eds. Statistical Methods in Diagnostic Medicine. New York:John Wiley \& Sons, Inc.;2002:274-306.

20. Alonzo TA, Pepe MS. Distribution-free ROC analysis using binary regression techniques. Biostatistics 2002;3:421-432.

21. Pepe MS. Three approaches to regression analysis of receiver operating characteristic curves for continuous test results. Biometrics 1998;54:124-135.

22. Pepe MS. An interpretation for the ROC curve and inference using GLM procedures. Biometrics 2000;56:352-359.

23. Pepe MS. The Statistical Evaluation of Medical Tests for Classification and Prediction. Oxford, UK: Oxford University Press; 2003:130-67.

24. Wei E, Jia $Y$, Tan $O$, et al. Parafoveal retinal vascular response to pattern visual stimulation assessed with OCT angiography. PLoS One 2013;8:e81343.

25. Gadde SG, Anegondi N, Bhanushali D, et al. Quantification of Vessel Density in Retinal Optical Coherence Tomography Angiography Images Using Local Fractal Dimension. Invest Ophthalmol Vis Sci 2016;57:246-252. 
26. Burgoyne CF, Downs JC, Bellezza AJ, Suh JK, Hart RT. The optic nerve head as a biomechanical structure: a new paradigm for understanding the role of IOP-related stress and strain in the pathophysiology of glaucomatous optic nerve head damage. Prog Retin Eye Res 2005;24:3973.

27. Lijmer JG, Mol BW, Heisterkamp S, et al. Empirical evidence of design-related bias in studies of diagnostic tests. JAMA 1999;282:1061-1066.

28. Medeiros FA, Ng D, Zangwill LM, Sample PA, Bowd C, Weinreb RN. The effects of study design and spectrum bias on the evaluation of diagnostic accuracy of confocal scanning laser ophthalmoscopy in glaucoma. Invest Ophthalmol Vis Sci 2007;48:214-222.

29. Rao HL, Kumbar T, Addepalli UK, et al. Effect of spectrum bias on the diagnostic accuracy of spectral-domain optical coherence tomography in glaucoma. Invest Ophthalmol Vis Sci 2012;53:1058-1065. 


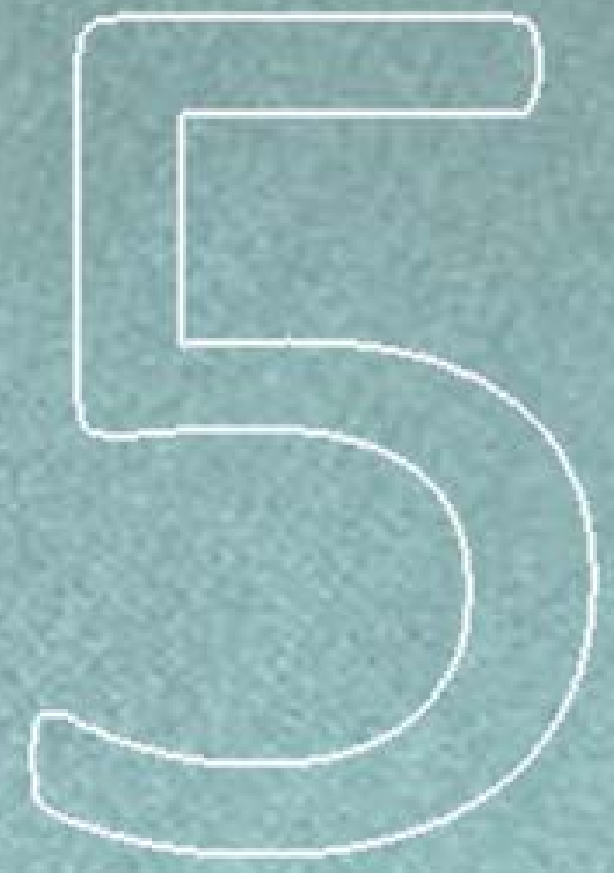

\section{Vessel density and structural measurements of OCT in POAG}

A comparison of the diagnostic ability of vessel density and structural measurements of optical coherence tomography in primary open angle glaucoma

Harsha L Rao, Zia S Pradhan, Robert N Weinreb, Mohammed Riyazuddin, Srilakshmi Dasari, Jayasree P Venugopal, Narendra K Puttaiah, Dhanaraj A S Rao, Sathi Devi, Kaweh Mansouri, Carroll AB Webers.

PLoS One 2017 Mar 13;12(3):e0173930 


\section{ABSTRACT}

Purpose: To compare the diagnostic abilities of vessel density measurements of the optic nerve head $(\mathrm{ONH})$, peripapillary and macular regions on optical coherence tomography (OCT) angiography in eyes with primary open angle glaucoma (POAG) with that of the $\mathrm{ONH}$ rim area, peripapillary retinal nerve fiber layer (RNFL) thickness and the macular ganglion cell complex (GCC) thickness measurements.

Methods: In a cross sectional study, 78 eyes of 50 control subjects and 117 eyes of 67 POAG patients underwent vessel density and structural measurements with spectral domain OCT. POAG was diagnosed based on the masked evaluation of optic disc stereo photographs. Area under receiver operating characteristic curves (AUC) and sensitivities at fixed specificities of vessel densities in $\mathrm{ONH}$, peripapillary and macular regions were compared with rim area, RNFL and GCC thickness.

Results: The AUC (sensitivity at 95\% specificity) of average vessel densities within the $\mathrm{ONH}$, peripapillary and macular region were 0.77 (31\%), 0.85 (56\%) and $0.70(18 \%)$ respectively. The same of ONH rim area, average RNFL and GCC thickness were 0.94 (83\%), 0.95 (72\%) and 0.93 (62\%) respectively. AUCs of vessel densities were significantly lower $(p<0.05)$ than that of the corresponding structural measurements. Pre-treatment IOP (coefficient: 0.08 ) affected $(p<0.05)$ the AUC of ONH vessel density but not of any other vessel density or structural measurements.

Conclusions: Diagnostic abilities of $\mathrm{ONH}$, peripapillary and the macular vessel densities in POAG were significantly lower than ONH rim area, peripapillary RNFL and macular GCC measurements respectively. At fixed levels of glaucoma severity, the diagnostic ability of the $\mathrm{ONH}$ vessel density was significantly greater in eyes with higher pre-treatment IOP. 


\section{INTRODUCTION}

Optical coherence tomography (OCT) angiography is a new technique of noninvasively imaging the blood vessels of the optic nerve head (ONH) and retina in-vivo. Of the multiple algorithms developed to achieve blood vessel delineation using the OCT platform, split spectrum amplitude-decorrelation angiography (SSADA) was the first one that was commercially available. ${ }^{1}$ Early studies using the SSADA algorithm have shown that the vessel density measurements provided by OCT angiography (OCTA) were repeatable and reproducible. ${ }^{2-6}$

Primary open angle glaucoma (POAG) is a chronic progressive optic neuropathy resulting from the apoptosis of the retinal ganglion cells (RGC). ${ }^{7}$ Evaluating the neuroretinal rim area, retinal nerve fiber layer (RNFL) and ganglion cell complex (GCC) thickness on OCT is used as a surrogate measure in clinical practice to estimate the amount of RGC loss in POAG. Although increased intraocular pressure (IOP) is the predominant risk factor for RGC death, ${ }^{8}$ reduced $\mathrm{ONH}$ perfusion has also been proposed to play a role in the pathogenesis of glaucoma. ${ }^{9}, 10$ Studies using the SSADA algorithm of OCTA have demonstrated reduced ONH and peripapillary vessel densities in patients with glaucoma. ${ }^{2-5,} 11,12$ In addition to reduced $\mathrm{ONH}$ and peripapillary vessel densities, we recently demonstrated reduced vessel densities in the macula of patients with POAG. ${ }^{13}$ Previous studies have compared the diagnostic ability of peripapillary vessel density measurements of OCTA with the RNFL thickness measurements of OCT. ${ }^{5,12}$ However, to the best of our knowledge, there are no studies comparing the diagnostic ability of inside disc vessel densities with $\mathrm{ONH}$ rim area or the macular vessel density with macular GCC thickness. The purpose of the current study was to compare the diagnostic abilities of the vessel density measurements of the $\mathrm{ONH}$, peripapillary and macular regions on OCTA in eyes with POAG with that of the ONH rim area, peripapillary RNFL thickness and the macular GCC thickness measurements on OCT. The secondary objective was to evaluate the effect of pre-treatment IOP on the diagnostic abilities of the vessel densities and structural measurements of OCT. 


\section{METHODS}

This was a prospective, cross-sectional study conducted at Narayana Nethralaya, a tertiary eye care center in Bengaluru, South India between September 2015 and July 2016. The methodology adhered to the tenets of the Declaration of Helsinki for research involving human subjects. Written informed consent was obtained from all participants and the study was approved by the Ethics Committee of Narayana Nethralaya (approval number: C/2015/08/04).

Participants of the study included control subjects and POAG patients. Control subjects were either hospital staff or subjects who consulted for a routine eye examination or a refractive error. Control subjects had no family history of glaucoma, IOP $\leq 21 \mathrm{~mm} \mathrm{Hg}$, open angles on gonioscopy, normal anterior and posterior segment on clinical examination by an ophthalmologist and non-glaucomatous optic discs, as assessed by glaucoma experts on masked examination of stereoscopic optic disc photographs. POAG patients had open angles on gonioscopy and glaucomatous changes on optic nerve head examination (neuroretinal rim narrowing, notching and retinal nerve fiber layer defects) as documented by glaucoma experts on dilated examination and confirmed by experts on stereoscopic optic disc photographs. Neither pre-treatment IOP, nor visual field changes were used to define POAG. Inclusion criteria for all participants were age $\geq 18$ years, corrected distance visual acuity of $20 / 40$ or better and refractive error within $\pm 5 \mathrm{D}$ sphere and $\pm 3 \mathrm{D}$ cylinder. Exclusion criteria were presence of any media opacities that prevented good quality OCT scans, or any retinal or neurological disease other than glaucoma, which could confound the evaluation. Eyes with a history of trauma or inflammation were also excluded. All participants underwent a comprehensive ocular examination, which included a detailed medical history, corrected distance visual acuity measurement, slitlamp biomicroscopy, Goldmann applanation tonometry, gonioscopy, dilated fundus examination, visual field (VF) examination and OCT imaging with RTVueXR SD-OCT (Optovue Inc., Fremont, CA). In addition to IOP measured on the day of scanning, the pre-treatment IOP (i.e. the IOP noted on the day of initiating anti-glaucoma treatment) was documented for all POAG eyes. 
Stereoscopic optic disc photographs were obtained by trained technicians using a digital fundus camera (Kowa nonmyd WX, Kowa Company, Ltd., Japan). Each optic disc photograph was evaluated independently by two glaucoma experts (HLR and NKP) in a masked manner to determine the presence of glaucomatous changes (focal or diffuse neuroretinal rim thinning, localized notching or RNFL defects). The experts were masked to all the clinical data, visual field data and the fellow eye data. Discrepancy in the classification between the two experts was adjudicated by a third glaucoma expert (ZSP).

VF examination was performed using a Humphrey Field analyzer II, model 720i (Zeiss Humphrey Systems, Dublin, CA), with the Swedish interactive threshold algorithm (SITA) standard 24-2 program. VFs were considered reliable if the fixation losses were less than $20 \%$, and the false positive and false negative response rates were less than $15 \%$. VF findings were not used for defining glaucoma or controls but were considered for the grading of glaucoma severity.

OCTA imaging of the optic disc region and macula was performed using RTVue-XR SD-OCT (AngioVue, v2015.100.0.33). The procedure of OCTA imaging with RTVue$\mathrm{XR}$ has been detailed previously. ${ }^{13}$ In brief, it uses an $840 \mathrm{~nm}$ diode laser source, with an A-scan rate of $70 \mathrm{kHz}$ per second. Imaging is performed using a set of 2 scans; one vertical priority and one horizontal priority raster volumetric scan. The optic disc scan covers an area of $4.5 \times 4.5 \mathrm{~mm}$ and the macular scan was performed using volumetric scans covering $3 \times 3 \mathrm{~mm}$. An orthogonal registration algorithm is used to produce merged 3-dimensional OCT angiograms. ${ }^{14}$ The SSADA algorithm compares the consecutive B-scans at the same location to detect flow using motion contrast, thereby delineating blood vessels. ${ }^{1}$ Vessel density is defined as the percentage area occupied by the large vessels and microvasculature in a particular region. Vessel densities are calculated over the entire scan area, i.e., whole enface disc and whole enface macula, as well as defined areas within each scan as described below. In addition, the software calculates vessel densities in various layers of the retina and the $\mathrm{ONH}$.

In the optic disc scan, the software automatically fits an ellipse to the optic disc margin and calculates the average vessel density within the ONH (referred to as 
the inside disc vessel density). The peripapillary region is defined as a $0.75 \mathrm{~mm}$ wide elliptical annulus extending from the optic disc boundary and the average vessel density with this region is calculated. Both the $\mathrm{ONH}$ and the peripapillary region are divided into 6 sectors based on the Garway-Heath map and the vessel densities in each sector is calculated (nasal, inferonasal, inferotemporal, superotemporal, superonasal and temporal sectors). ${ }^{15}$ In order to compare the inside disc and peripapillary vessel densities with the rim area and the RNFL measurements of OCT, the superonasal and superotemporal sectors were combined together as the superior sector, and the inferonasal and inferotemporal sectors as the inferior sector. For each scanned region, the software calculates the vessel densities in various layers of the retina and $\mathrm{ONH}$. For the purpose of this study, the antero-posterior segment used for each region is as follows. The $\mathrm{ONH}$ vessel densities were calculated from the "nerve head segment" of the $\mathrm{ONH}$ angiogram. This segment extends from 2000 microns above the internal limiting membrane (ILM) to 150 microns below the ILM. The peripapillary vessel density was analyzed from the "Radial Peripapillary Capillary (RPC) segment" which extends from the ILM to the posterior boundary of the nerve fiber layer. Macular vessel densities were analyzed over a $1.5 \mathrm{~mm}$-wide parafoveal, circular annulus centered on the macula. The parafoveal region was also divided into 4 sectors of $90^{\circ}$ each (nasal, inferior, superior and temporal sectors). Macular vessel densities analyzed in this study were of the superficial vascular plexus present in the inner layers of the retina (extending from the internal limiting membrane to the inner plexiform layer).

All subjects also underwent the traditional ONH, peripapillary RNFL and macular GCC thickness measurements on RTVue-XR SD-OCT using the ONH and the GCC scans. These scan protocols have been explained in detail previously. ${ }^{16,17}$ All the examinations for a particular subject were performed on the same day. Image quality was assessed for all OCTA and OCT scans. Poor quality images, which were defined as those with a signal strength index (SSI) less than 35 or images with motion artifacts and segmentation errors were excluded from the analysis. Figure 1 shows the OCTA and the OCT maps of a normal eye and an eye with POAG. 
(a) Nerve head segment
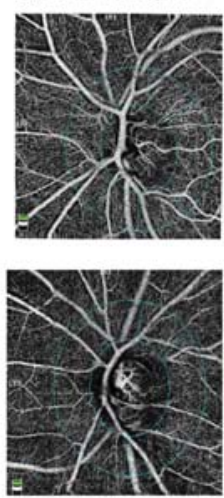

(b) RPC segment
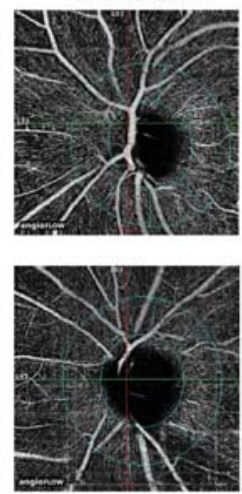

(c) Macular region
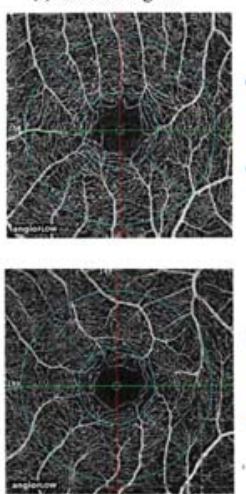

(d) RNFL map
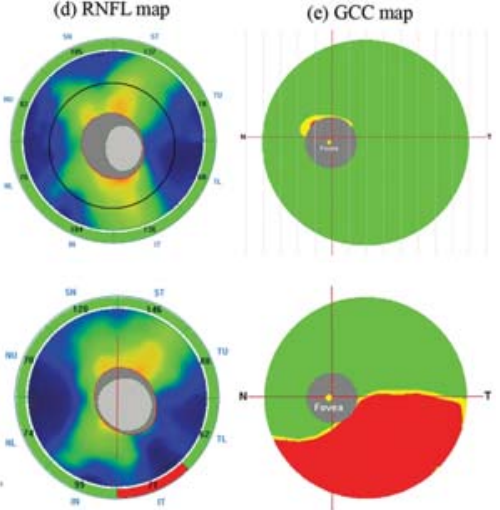

Figure 1. Nerve head segment (a), radial peripapillary capillary, RPC segment (b) and macular (c) optical coherence tomography angiography scans of a normal eye (top panel) and an eye with glaucoma (bottom panel). The figure also shows the retinal nerve fiber layer, RNFL (d) and ganglion cell complex, GCC (e) maps of the two eyes. Vessel loss in the eye with glaucoma can be noted in the inferotemporal peripapillary region correlating with the RNFL loss seen on the RNFL map.

\section{STATISTICAL ANALYSIS}

Descriptive statistics included mean and standard deviation for normally distributed variables and median and inter-quartile range (IQR) for non-normally distributed variables. Shapiro-Wilk test was used to test for the normality distribution of continuous variables. Normally distributed continuous variables between the control and the glaucoma groups were compared using t test. Nonnormally distributed continuous variables were compared using Wilcoxon rank sum test. Percentages were compared using Chi square test. Receiver operating characteristic (ROC) curves were used to describe the ability of vessel density and structural measurements of OCT to discriminate glaucomatous eyes from control eyes. Sensitivities at fixed specificities of $80 \%$ and $95 \%$ were determined for all the parameters. To obtain confidence intervals for area under the ROC curves (AUC) and sensitivities, a bootstrap re-sampling procedure was used $(n=1000$ re-samples). As measurements from both eyes of the same subject are likely to be correlated, the standard statistical methods for parameter estimation can lead 
to underestimation of standard errors and to confidence intervals that are too narrow. ${ }^{18}$ Therefore, the cluster of data for the study subject was considered as the units of resampling and bias corrected standard errors were calculated during all estimations. This procedure has been used to adjust for the presence of multiple correlated measurements from the same unit. ${ }^{19,} 20$ To compare the AUCs, a Wald statistic, dividing the observed AUC difference by its standard error, was compared with the standard normal distribution and a $p$ value was reported. ROC regression modeling technique was used to evaluate the effect of glaucoma severity and the pre-treatment IOP on the AUCs and sensitivities of OCT measurements in diagnosing glaucoma. ${ }^{21,22}$

Statistical analyses were performed using commercial software (Stata ver. 13.1; StataCorp, College Station, TX). A two-tailed $p$ value of $\leq 0.05$ was considered statistically significant.

\section{RESULTS}

Two hundred and twenty-two eyes of 123 subjects (78 eyes of 50 normal and 144 eyes of 73 POAG subjects) underwent vessel density and structural imaging with OCT. Among these, 25 eyes of 18 POAG patients in which the optic disc classification on stereo photographs was not glaucomatous optic neuropathy, were excluded. Of the remaining eyes, 8 eyes with unreliable VF, 19 eyes with poor OCTA scans of ONH, 25 eyes with poor OCTA scans of macula, 17 eyes with poor structural scans of $\mathrm{ONH}$ and 2 eyes with poor GCC scans were excluded. Final analysis included vessel density and structural scans from 195 eyes of 117 subjects (78 eyes of 50 normal and 117 eyes of 67 POAG subjects). Of the 117 eyes with POAG, 22 eyes had a "within normal limit" or a "borderline" glaucoma hemifield test result, and / or the probability value of pattern standard deviation $>5 \%$ on VF (preperimetric glaucoma). Table 1 shows the clinical, VF, vessel density and structural measurements of the included subjects. SSI of the OCTA and structural scan of ONH were significantly greater in the control subjects compared to the POAG patients. AUCs and sensitivities at fixed specificities of optic disc and peripapillary vessel density and structural parameters were therefore calculated 
after adjusting for the difference in signal strength between the control and POAG groups using covariate-adjustment as proposed by Pepe. ${ }^{23}$ All the vessel density and structural measurements were significantly lesser in the glaucoma compared to the control group.

Table 1. Clinical features, visual field parameters, vessel density and structural measurements of the participants. All values represent median and interquartile range unless specified.

\begin{tabular}{|c|c|c|c|}
\hline & $\begin{array}{l}\text { Control group } \\
\text { (78 eyes } \\
50 \text { subjects) }\end{array}$ & $\begin{array}{l}\text { POAG group } \\
\text { (117 eyes } \\
67 \text { patients) }\end{array}$ & $\mathbf{P}$ \\
\hline Age (years)* & $60.7 \pm 8.3$ & $62.8 \pm 12.1$ & 0.30 \\
\hline Gender (male:female) & $27: 23$ & $47: 20$ & 0.07 \\
\hline Sphere (D) & $0.5(0,1)$ & $0.0(-0.75,0.75)$ & 0.12 \\
\hline Cylinder (D) & $-0.5(-1,-0.5)$ & $-0.75(-1,-0.5)$ & 0.72 \\
\hline Pseudophakia (n, \%) & $13(16.7 \%)$ & $29(24.8 \%)$ & 0.39 \\
\hline Optic disc area $\left(\mathrm{mm}^{2}\right)$ & $2.28(1.93,2.53)$ & $2.27(2.01,2.59)$ & 0.45 \\
\hline Pre-treatment IOP (mm Hg) & & $20(18,24.5)$ & \\
\hline IOP at the scanning visit (mm Hg) & $15.5(14,18)$ & $16(14,19)$ & 0.001 \\
\hline Hypertension (yes:no) & 19:31 & 29:38 & 0.57 \\
\hline Diabetes mellitus (yes:no) & $17: 33$ & $20: 47$ & 0.63 \\
\hline Mean deviation (dB) & $-0.9(-3.5,-0.3)$ & $-6.3(-12.5,-3.5)$ & $<0.001$ \\
\hline Pattern standard deviation (dB) & $1.9(1.5,2.5)$ & $4.8(2.6,9.7)$ & $<0.001$ \\
\hline Visual field index (\%) & $99(97,99)$ & $88(69,95)$ & $<0.001$ \\
\hline \multicolumn{4}{|c|}{ OCTA parameters } \\
\hline SSI (Optic disc scan)* & $53.6 \pm 8.9$ & $49.6 \pm 7.8$ & 0.002 \\
\hline Whole enface vessel density (disc scan, \%) & $53.9(51.3,55.5)$ & $45.2(41.5,48.7)$ & $<0.001$ \\
\hline Inside disc vessel density (\%) & $48.1(44.0,50.0)$ & $40.1(34.8,45.63)$ & $<0.001$ \\
\hline Nasal vessel density (\%) & $48.9(44.1,52.7)$ & $41.2(33.6,47.0)$ & $<0.001$ \\
\hline Inferior vessel density (\%) & $48.7(44.8,53.3)$ & $43.8(37.5,49.5)$ & $<0.001$ \\
\hline Superior vessel density (\%) & $49.1(43.4,52.2)$ & $40.8(33.2,46.2)$ & $<0.001$ \\
\hline Temporal vessel density (\%) & $44.3(40.4,50.7)$ & $34.9(28.8,43.6)$ & $<0.001$ \\
\hline Average Peripapillary vessel density (\%) & $61.9(59.9,64.2)$ & $54.4(49.0,58.6)$ & $<0.001$ \\
\hline Nasal vessel density (\%) & $59.5(57.0,61.8)$ & $53.2(46.9,57.4)$ & $<0.001$ \\
\hline Inferior vessel density (\%) & $64.7(62.1,67.2)$ & $53.9(45.6,59.5)$ & $<0.001$ \\
\hline Superior vessel density (\%) & $63.6(60.3,66.9)$ & $55.2(49.4,60.7)$ & $<0.001$ \\
\hline Temporal vessel density (\%) & $60.3(58.0,63.2)$ & $56.5(51.3,59.8)$ & $<0.001$ \\
\hline SSI (Macula scan)* & $61.1 \pm 6.7$ & $59.9 \pm 7.7$ & 0.33 \\
\hline Whole enface vessel density (macula scan, \%) & $47.1(45.5,50.1)$ & $43.8(41.8,47.0)$ & $<0.001$ \\
\hline Parafoveal vessel density (\%) & $49.5(47.3,52.3)$ & $46.9(43.9,49.4)$ & $<0.001$ \\
\hline Nasal vessel density (\%) & $48.3(46.4,51.1)$ & $45.9(43.1,48.8)$ & 0.001 \\
\hline Inferior vessel density (\%) & $51.0(48.0,53.3)$ & $46.6(43.7,51.1)$ & $<0.001$ \\
\hline
\end{tabular}




\begin{tabular}{lccc} 
Superior vessel density (\%) & $50.3(48.1,53.2)$ & $47.5(44.3,51.6)$ & 0.002 \\
Temporal vessel density $(\%)$ & $49.6(46.8,52.5)$ & $46.8(44.1,49.7)$ & $<0.001$ \\
\hline & OCT parameters & & $<0.001$ \\
\hline SSI (ONH scan) & $55.2 \pm 8.1$ & $49.4 \pm 8.3$ & $<0.001$ \\
Neuroretinal rim area $\left(\mathrm{mm}^{2}\right)$ & $1.32(1.12,1.51)$ & $0.72(0.56,0.88)$ & $<0.001$ \\
Nasal rim area $\left(\mathrm{mm}^{2}\right)$ & $0.40(0.34,0.44)$ & $0.21(0.15,0.28)$ & $<0.001$ \\
Inferior rim area $\left(\mathrm{mm}^{2}\right)$ & $0.41(0.33,0.48)$ & $0.16(0.10,0.26)$ & $<0.001$ \\
Superior rim area $\left(\mathrm{mm}^{2}\right)$ & $0.38(0.32,0.48)$ & $0.22(0.17,0.28)$ & $<0.001$ \\
Temporal rim area $\left(\mathrm{mm}^{2}\right)$ & $0.12(0.09,0.19)$ & $0.09(0.06,0.14)$ & $<0.001$ \\
Average Peripapillary RNFL thickness $(\mu \mathrm{m})$ & $100(93,105)$ & $79(70,85)$ & $<0.001$ \\
Nasal RNFL thickness $(\mu \mathrm{m})$ & $80(73,87)$ & $66(58,73)$ & $<0.001$ \\
Inferior RNFL thickness $(\mu \mathrm{m})$ & $123(116,132)$ & $86(70,98)$ & $<0.001$ \\
Superior RNFL thickness $(\mu \mathrm{m})$ & $124(115,133)$ & $97(83,107)$ & $<0.001$ \\
Temporal RNFL thickness $(\mu \mathrm{m})$ & $71(66,79)$ & $63(57,69)$ & 0.78 \\
SSI (GCC scan) & $59.1 \pm 9.7$ & $58.7 \pm 9.5$ & $<0.001$ \\
Average GCC thickness $(\mu \mathrm{m})$ & $95(91,102)$ & $79(72,85)$ & $<0.001$ \\
Superior GCC thickness $(\mu \mathrm{m})$ & $95(90,102)$ & $82(74,89)$ & $<0.001$ \\
Inferior GCC thickness $(\mu \mathrm{m})$ & $96(91,102)$ & $77(66,84)$ &
\end{tabular}

POAG: primary open angle glaucoma; D: diopter; dB: decibel; IOP: intraocular pressure; SSI: signal strength index; ONH: optic nerve head; RNFL: retinal nerve fiber layer; GCC: ganglion cell complex; *mean \pm standard deviation.

The AUCs and sensitivities at fixed specificities of the vessel density measurements to differentiate POAG from control eyes are shown in Table 2. Whole enface vessel density of the disc scan showed the best AUC and sensitivity at fixed specificity to diagnose glaucoma. The AUCs and sensitivities at fixed specificities of the structural measurements to differentiate POAG from control eyes are shown in Table 3. Average and the inferior quadrant RNFL thickness showed the best AUC and sensitivity at fixed specificity to diagnose glaucoma. Comparing the diagnostic abilities region-wise, ONH rim area showed a statistically significantly better $(p<0.001)$ AUC than the inside disc vessel density, peripapillary RNFL thickness showed a statistically significantly better $(p=0.002) A U C$ than the peripapillary vessel density, and macular GCC thickness showed a statistically significantly better $(p<0.001)$ AUC than the macular vessel density. Figure 2 shows the sensitivity at $95 \%$ specificity of the vessel density and structural measurements at different severities of glaucomatous VF loss. Sensitivities of structural measurements were better than the vessel densities of the corresponding regions over the whole range of glaucoma severity. 
Table 2. Diagnostic ability of vessel density parameters of optical coherence tomography angiography in differentiating open angle glaucoma from control eyes (figures in parenthesis represent $95 \%$ confidence intervals).

\begin{tabular}{lccc}
\hline \multicolumn{1}{c}{ Vessel density } & AUC & $\begin{array}{c}\text { Sensitivity at } \\
95 \% \text { specificity }\end{array}$ & $\begin{array}{c}\text { Sensitivity at 80\% } \\
\text { specificity }\end{array}$ \\
\hline Whole enface (disc) & $0.93(0.88-0.96)$ & $75 \%(48-89)$ & $88 \%(75-95)$ \\
Inside disc & $0.77(0.67-0.86)$ & $31 \%(08-60)$ & $58 \%(36-74)$ \\
$\quad$ Nasal & $0.74(0.65-0.83)$ & $19 \%(01-41)$ & $57 \%(28-70)$ \\
Inferior & $0.67(0.57-0.77)$ & $25 \%(06-44)$ & $46 \%(27-61)$ \\
Superior & $0.73(0.62-0.81)$ & $23 \%(04-45)$ & $47 \%(28-69)$ \\
$\quad$ Temporal & $0.70(0.56-0.81)$ & $13 \%(03-53)$ & $47 \%(13-62)$ \\
Average Peripapillary & $0.85(0.78-0.90)$ & $56 \%(39-70)$ & $76 \%(62-87)$ \\
$\quad$ Nasal & $0.78(0.68-0.85)$ & $40 \%(29-59)$ & $62 \%(46-79)$ \\
Inferior & $0.88(0.81-0.92)$ & $66 \%(47-83)$ & $78 \%(66-90)$ \\
Superior & $0.82(0.73-0.88)$ & $52 \%(35-68)$ & $66 \%(51-77)$ \\
$\quad$ Temporal & $0.68(0.57-0.77)$ & $26 \%(08-52)$ & $45 \%(28-57)$ \\
Whole enface (macula scan) & $0.73(0.64-0.81)$ & $18 \%(01-40)$ & $57 \%(37-72)$ \\
Parafoveal & $0.70(0.61-0.78)$ & $10 \%(03-36)$ & $49 \%(34-64)$ \\
$\quad$ Nasal & $0.65(0.55-0.74)$ & $08 \%(01-27)$ & $50 \%(28-64)$ \\
Inferior & $0.69(0.60-0.77)$ & $19 \%(04-41)$ & $52 \%(33-64)$ \\
Superior & $0.65(0.55-0.74)$ & $15 \%(03-38)$ & $42 \%(15-60)$ \\
Temporal & $0.67(0.58-0.76)$ & $12 \%(02-26)$ & $42 \%(18-66)$ \\
\hline
\end{tabular}

AUC: area under the receiver operating characteristic curve.

Table 3. Diagnostic ability of structural parameters of optical coherence tomography in differentiating open angle glaucoma from control eyes (figures in parenthesis represent $95 \%$ confidence intervals).

\begin{tabular}{lccc}
\hline \multicolumn{1}{c}{ Vessel density } & AUC & $\begin{array}{c}\text { Sensitivity at } \\
\mathbf{9 5 \%} \text { specificity }\end{array}$ & $\begin{array}{c}\text { Sensitivity at } \\
\mathbf{8 0 \%} \text { specificity }\end{array}$ \\
\hline Neuroretinal rim area & $0.94(0.88-0.98)$ & $83 \%(70-95)$ & $89 \%(78-97)$ \\
Nasal rim area & $0.88(0.81-0.94)$ & $58 \%(38-73)$ & $79 \%(63-89)$ \\
Inferior rim area & $0.92(0.85-0.96)$ & $73 \%(57-82)$ & $84 \%(72-93)$ \\
Superior rim area & $0.89(0.83-0.94)$ & $65 \%(50-83)$ & $86 \%(75-95)$ \\
Temporal rim area & $0.72(0.61-0.82)$ & $26 \%(09-38)$ & $52 \%(34-74)$ \\
Average RNFL thickness & $0.95(0.91-0.98)$ & $72 \%(45-88)$ & $94 \%(85-99)$ \\
Nasal RNFL thickness & $0.83(0.73-0.90)$ & $44 \%(03-65)$ & $69 \%(46-86)$ \\
Inferior RNFL thickness & $0.95(0.91-0.98)$ & $84 \%(72-92)$ & $90 \%(81-96)$ \\
Superior RNFL thickness & $0.90(0.83-0.94)$ & $56 \%(38-72)$ & $81 \%(63-90)$
\end{tabular}




\begin{tabular}{clll} 
Temporal RNFL thickness & $0.73(0.63-0.82)$ & $24 \%(13-46)$ & $51 \%(26-69)$ \\
Average GCC thickness & $0.93(0.88-0.96)$ & $62 \%(47-83)$ & $87 \%(75-95)$ \\
Superior GCC thickness & $0.86(0.79-0.91)$ & $41 \%(28-63)$ & $77 \%(61-88)$ \\
Inferior GCC thickness & $0.92(0.87-0.95)$ & $63 \%(49-74)$ & $86 \%(70-93)$ \\
\hline
\end{tabular}

AUC: area under the receiver operating characteristic curve; RNFL: retinal nerve fiber layer; GCC: ganglion cell complex.
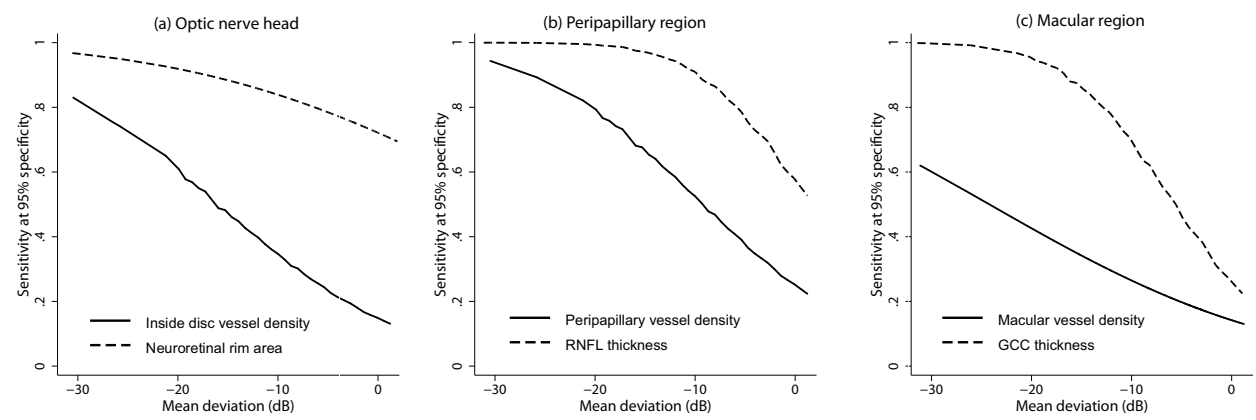

Figure 2. Sensitivity at $95 \%$ specificity of (a) optic nerve head vessel density and rim area, (b) peripapillary vessel density and retinal nerve fiber layer (RNFL) thickness and (c) superficial macular vessel density and ganglion cell complex (GCC) thickness according to mean deviation on visual fields.

Table 4 shows the effect of pre-treatment IOP on the diagnostic abilities of vessel density and structural parameters of the three regions, after adjusting for the disease severity as determined by the MD of the VF. Pre-treatment IOP had a significant positive effect on the AUC of inside disc vessel density but not on any other vessel density or structural measurement. AUC and sensitivities at fixed specificities of inside disc vessel density increased significantly in eyes with higher pre-treatment IOPs. Figure 3 shows the effect of pre-treatment IOP on the sensitivities at $95 \%$ specificity of the inside disc vessel density and rim area measurements at a MD value of $-5 \mathrm{~dB}$. 
Table 4. Results of the multivariate receiver operating characteristic (ROC) regression models evaluating the effect of mean deviation of visual fields and pre-treatment intraocular pressure (IOP) on the area under the ROC curves of the vessel density and structural measurements inside the optic disc, peripapillary and parafoveal region. Figures represent coefficient with $95 \%$ confidence interval in parenthesis.

\begin{tabular}{ccc}
\hline Parameter & Mean deviation & Pre-treatment IOP \\
\hline $\begin{array}{c}\text { Optic nerve head } \\
\text { Vessel density }\end{array}$ & $-0.06(-0.12,-0.01)^{*}$ & $0.08(0.03,0.16)^{*}$ \\
$\quad$ Rim area & $-0.04(-0.12,0.08)$ & $0.01(-0.06,0.23)$ \\
Peripapillary region & & \\
$\quad$ Vessel density & $-0.07(-0.14,-0.01)^{*}$ & $0.02(-0.02,0.08)$ \\
RNFL thickness & $-0.14(-0.34,-0.05)^{*}$ & $-0.06(-0.22,0.05)$ \\
Parafoveal region & & \\
$\quad$ Vessel density & $-0.05(-0.10,0.00)^{*}$ & $-0.01(-0.04,0.04)$ \\
GCC thickness & $-0.12(-0.23,-0.04)^{*}$ & $-0.01(-0.07,0.04)$ \\
\hline
\end{tabular}

* - statistically significant $(p<0.05)$. RNFL: retinal nerve fiber layer; GCC: ganglion cell complex.

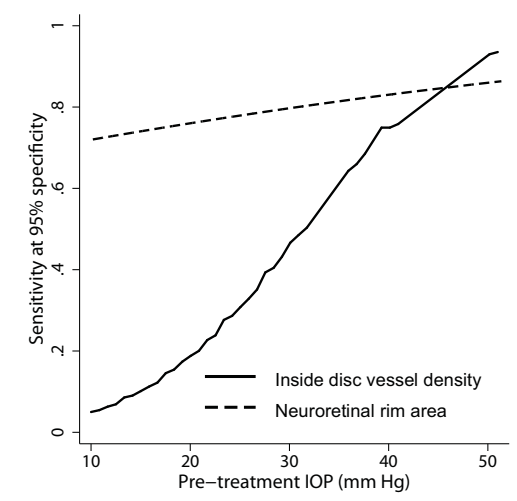

Figure 3 . Sensitivity at $95 \%$ specificity of optic nerve head vessel density and rim area according to the pre-treatment intraocular pressure at a mean deviation on visual fields of $-5 \mathrm{~dB}$.

We ran the entire analysis considering one eye of subjects who contributed both eyes for our earlier analysis and found similar results. When considering the better eye of the glaucoma patients for analysis (median MD: $-4.3 \mathrm{~dB}$ ), the AUC of inside disc (0.74), peripapillary (0.82) and parafoveal (0.70) vessel densities 
were significantly lower ( $p<0.01$ for all comparisons) than $\mathrm{ONH}$ rim area (0.93), peripapillary RNFL (0.93) and average GCC thickness (0.89) respectively. When considering the worse eye of the glaucoma patients for analysis (median MD: $-8.5 \mathrm{~dB}$ ), the AUC of inside disc (0.78), peripapillary (0.88) and parafoveal (0.74) vessel densities were similarly significantly lower ( $p<0.02$ for all comparisons) than ONH rim area (0.93), peripapillary RNFL (0.96) and average GCC thickness (0.95) respectively.

We also ran the entire analysis considering optic disc changes and VF changes as the definition of glaucoma (excluding preperimetric glaucoma eyes) and found similar results. The AUC of inside disc (0.79), peripapillary (0.88) and parafoveal (0.72) vessel densities were still significantly lower ( $p<0.01$ for all comparisons) than ONH rim area (0.96), peripapillary RNFL (0.97) and average GCC thickness (0.96) respectively.

\section{DISCUSSION}

In this study, vessel density measurements of OCTA were compared with structural measurements of the traditional OCT. It was found that the diagnostic abilities of several OCT parameters (ONH rim area, peripapillary RNFL thickness and the macular GCC thickness) in POAG were significantly better than the corresponding vessel densities within each of these regions.

Previous studies have compared the diagnostic ability of peripapillary vessel density measurements of OCTA with the RNFL thickness measurements of OCT. ${ }^{5}$, 12 However, to the best of our knowledge, there are no studies comparing the inside disc vessel densities with $\mathrm{ONH}$ rim area or the macular vessel densities with macular GCC thickness. Liu et al evaluated the diagnostic ability of peripapillary vessel density and average RNFL thickness in 12 (9 perimetric and 3 pre-perimetric) glaucoma and 12 normal eyes. AUC, sensitivity and specificity of peripapillary vessel density $(0.94,83.3 \%$ and $91.7 \%$ respectively) were found to be comparable to that of the average RNFL thickness $\left(0.97,91.7 \%\right.$ and $91.7 \%$ respectively). ${ }^{5}$ 
Yarmohammadi et al compared the diagnostic ability of peripapillary vessel density with that of the average RNFL thickness in 124 eyes with POAG (median MD: $-3.9 \mathrm{~dB}) .{ }^{12}$ Although the AUC of peripapillary vessel density measurement (0.83) was less than that of the average RNFL thickness (0.92), this difference was not statistically significant. Whole enface vessel density of the disc scan showed the best AUC in their study (AUC: 0.94), similar to that found in our study (0.93). ${ }^{12}$ We found slightly greater AUCs of the peripapillary vessel densities and the RNFL thickness (compared to the results of the study by Yarmohammadi et $\mathrm{al}^{12}$ ) owing to eyes with more advanced glaucoma in our cohort (median MD: $-6.3 \mathrm{~dB}$ ). Additionally, we found that the AUC of average RNFL thickness was significantly greater than that of the peripapillary vessel density measurement. Glaucoma in our study was defined solely on the neuroretinal rim and RNFL changes on clinical examination and stereo photographs of the optic discs. This may have biased the diagnostic ability of the OCT rim area and RNFL thickness measurements and could have been the reason for the better diagnostic ability of structural measurements compared to vessel density measurements. We therefore ran a separate analysis considering optic disc changes and VF changes as the definition of glaucoma and found the results to be the same. Also, the diagnostic ability of macular measurements is less likely to be influenced by the reference standard. Therefore, the results of our study is likely to represent true superiority of structural measurements over vessel density measurements for diagnosing glaucoma. Future studies with functional tests as reference standard and longitudinal evaluation of suspect eyes are required to validate our results.

As expected, the diagnostic abilities of vessel densities and the structural measurements increased with increasing severity of glaucoma. This has been reported earlier both with vessel density ${ }^{4,13}$ and with structural measurements. ${ }^{17}$ We therefore accounted for the severity of disease when evaluating for the effect of baseline IOP on the diagnostic abilities of vessel density and structural measurements. If reduced ONH blood supply was the predominant pathogenic mechanism in POAG eyes with low baseline IOPs (normal tension glaucoma, NTG), a greater difference in the vessel density values between the glaucoma and the control groups (and thereby a greater AUC) in these NTG eyes would be expected. 
However, this was not observed. On the contrary, the diagnostic ability of $\mathrm{ONH}$ vessel density increased in eyes with higher baseline IOP. This may imply that the vascular mechanisms contributing to the pathogenesis of glaucoma are not IOPindependent. IOP related stress and strain have been hypothesized to occlude the capillaries especially in the lamina cribrosa of the $\mathrm{ONH}^{24}$ Although there are no studies evaluating the effect of baseline IOP on the diagnostic abilities of the structural and vascular measurements of OCT as done in the current study, two previous studies have compared the diagnostic abilities of structural parameters of OCT in NTG (defined as open angle glaucoma eyes with baseline IOP $<21 \mathrm{~mm}$ $\mathrm{Hg}$ ) and POAG (defined as open angle glaucoma eyes with baseline $1 \mathrm{OP}>=21 \mathrm{~mm}$ $\mathrm{Hg}$ ) patients with comparable glaucoma severity as defined on the VFs. These studies have found that the diagnostic ability of RNFL thickness and GCC thickness was greater in POAG compared to NTG. ${ }^{25}, 26$ Contrary to the results of these studies, we found no statistically significant effect of the baseline IOP on the diagnostic abilities of RNFL and GCC thickness.

There are some limitations of the OCTA technology and the study design which need to be considered while interpreting the results. The vessel density measurements evaluated in this study were the ones provided by the software automatically. We therefore could not exactly match the vessel density sectors with the sectors of the structural parameters for comparison. The OCTA algorithm, in its current form, includes large vessels along with capillaries in its estimation of vessel density. The software also does not provide further insights into the nature of vascular changes such as attenuation, drop-out, etc. The technology also does not evaluate the choroidal vasculature. These details would provide a better understanding of the vascular changes in glaucoma. Another possible limitation of the current study was that we did not measure the blood pressure of the subjects or record their anti-hypertensive medication. However, we recorded the history of hypertension and found that the number of subjects with hypertension was similar in the glaucoma and the control groups. A previous study also has shown no relationship between blood pressure readings and peripapillary vessel densities on OCTA. ${ }^{5}$ In the same context, the peripapillary vessel densities can also be affected by parapapillary atrophy (PPA). ${ }^{27}$ We did not record the presence of PPA or its extent in our subjects. Another limitation of the study is the case- 
control design, with a clear distinction between glaucoma patients (cases) defined based on the presence of glaucomatous optic nerve head changes, and normal subjects (controls) with no suspicious findings of glaucoma. Such a design has been shown to overestimate the actual diagnostic ability of a test. ${ }^{28-30}$

In conclusion, we found that the diagnostic abilities of OCTA vessel density measurements of the $\mathrm{ONH}$, peripapillary and the macular regions in POAG were significantly lower than the OCT ONH rim area, peripapillary RNFL thickness and the macular GCC thickness measurements, respectively. At fixed levels of glaucoma severity, the diagnostic ability of the OCTA ONH vessel density was significantly greater in eyes with higher baseline IOP. Baseline IOP did not affect the diagnostic ability of the other OCTA vessel density or the OCT structural measurements.

\section{REFERENCES}

1. Jia Y, Tan O, Tokayer J, Potsaid B, Wang Y, Liu JJ, et al. Split-spectrum amplitude-decorrelation angiography with optical coherence tomography. Opt Express 2012;20:4710-25.

2. Jia Y, Morrison JC, Tokayer J, Tan O, Lombardi L, Baumann B, et al. Quantitative OCT angiography of optic nerve head blood flow. Biomed Opt Express 2012;3:3127-37.

3. Jia Y, Wei E, Wang X, Zhang X, Morrison JC, Parikh M, et al. Optical coherence tomography angiography of optic disc perfusion in glaucoma. Ophthalmology 2014;121:1322-32.

4. Wang X, Jiang C, Ko T, Kong X, Yu X, Min W, et al. Correlation between optic disc perfusion and glaucomatous severity in patients with open-angle glaucoma: an optical coherence tomography angiography study. Graefes Arch Clin Exp Ophthalmol 2015;253:1557-64.

5. Liu L, Jia Y, Takusagawa HL, Pechauer AD, Edmunds B, Lombardi L, et al. Optical Coherence Tomography Angiography of the Peripapillary Retina in Glaucoma. JAMA Ophthalmol 2015;133:1045-52.

6. Yu J, Jiang C, Wang X, Zhu L, Gu R, Xu H, et al. Macular perfusion in healthy Chinese: an optical coherence tomography angiogram study. Invest Ophthalmol Vis Sci 2015;56:3212-7.

7. Weinreb RN, Khaw PT. Primary open-angle glaucoma. Lancet 2004;363:1711-20.

8. Weinreb RN, Aung T, Medeiros FA. The pathophysiology and treatment of glaucoma: a review. JAMA 2014;311:1901-11.

9. Bonomi L, Marchini G, Marraffa M, Bernardi P, Morbio R, Varotto A. Vascular risk factors for primary open angle glaucoma: the Egna-Neumarkt Study. Ophthalmology 2000;107:128793. 
10. Leske MC, Heijl A, Hyman L, Bengtsson B, Dong L, Yang Z, et al. Predictors of long-term progression in the Early Manifest Glaucoma Trial. Ophthalmology 2007;114:1965-72.

11. Leveque PM, Zeboulon P, Brasnu E, Baudouin C, Labbe A. Optic Disc Vascularization in Glaucoma: Value of Spectral-Domain Optical Coherence Tomography Angiography. J Ophthalmol 2016;2016:6956717.

12. Yarmohammadi A, Zangwill LM, Diniz-Filho A, Suh MH, Manalastas PI, Fatehee N, et al. Optical Coherence Tomography Angiography Vessel Density in Healthy, Glaucoma Suspect, and Glaucoma Eyes. Invest Ophthalmol Vis Sci 2016;57:OCT451-9.

13. Rao HL, Pradhan ZS, Weinreb RN, Reddy HB, Riyazuddin M, Dasari S, et al. Regional Comparisons of Optical Coherence Tomography Angiography Vessel Density in Primary OpenAngle Glaucoma. Am J Ophthalmol 2016;171:75-83.

14. Kraus MF, Potsaid B, Mayer MA, Bock R, Baumann B, Liu JJ, et al. Motion correction in optical coherence tomography volumes on a per A-scan basis using orthogonal scan patterns. Biomed Opt Express 2012;3:1182-99.

15. Garway-Heath DF, Poinoosawmy D, Fitzke FW, Hitchings RA. Mapping the visual field to the optic disc in normal tension glaucoma eyes. Ophthalmology 2000;107:1809-15.

16. Rao HL, Zangwill LM, Weinreb RN, Sample PA, Alencar LM, Medeiros FA. Comparison of different spectral domain optical coherence tomography scanning areas for glaucoma diagnosis. Ophthalmology 2010;117:1692-9.

17. Rao HL, Leite MT, Weinreb RN, Zangwill LM, Alencar LM, Sample PA, et al. Effect of disease severity and optic disc size on diagnostic accuracy of RTVue spectral domain optical coherence tomograph in glaucoma. Invest Ophthalmol Vis Sci 2011;52:1290-6.

18. Glynn RJ, Rosner B. Accounting for the correlation between fellow eyes in regression analysis. Arch Ophthalmol 1992;110:381-7.

19. Zhou XH, Obuchowski NA, McClish DK. Analysis of correlated ROC data. In: Zhou XH, Obuchowski NA, McClish DK, eds. Statistical Methods in Diagnostic Medicine. New York:John Wiley \& Sons, Inc..;2002:274-306.

20. Alonzo TA, Pepe MS. Distribution-free ROC analysis using binary regression techniques. Biostatistics 2002;3:421-32.

21. Pepe MS. Three approaches to regression analysis of receiver operating characteristic curves for continuous test results. Biometrics 1998;54:124-35.

22. Pepe MS. An interpretation for the ROC curve and inference using GLM procedures. Biometrics 2000;56:352-9.

23. Pepe MS. The Statistical Evaluation of Medical Tests for Classification and Prediction. Oxford, UK: Oxford University Press; 2003:130-67.

24. Burgoyne CF, Downs JC, Bellezza AJ, Suh JK, Hart RT. The optic nerve head as a biomechanical structure: a new paradigm for understanding the role of IOP-related stress and strain in the pathophysiology of glaucomatous optic nerve head damage. Prog Retin Eye Res 2005;24:3973.

25. Kim NR, Hong S, Kim JH, Rho SS, Seong GJ, Kim CY. Comparison of macular ganglion cell complex thickness by Fourier-domain OCT in normal tension glaucoma and primary openangle glaucoma. J Glaucoma 2013;22:133-9. 
26. Firat PG, Doganay S, Demirel EE, Colak C. Comparison of ganglion cell and retinal nerve fiber layer thickness in primary open-angle glaucoma and normal tension glaucoma with spectraldomain OCT. Graefes Arch Clin Exp Ophthalmol. 2013;251:831-8.

27. Akagi T, lida $\mathrm{Y}$, Nakanishi $\mathrm{H}$, Terada N, Morooka S, Yamada $\mathrm{H}$, et al. Microvascular Density in Glaucomatous Eyes With Hemifield Visual Field Defects: An Optical Coherence Tomography Angiography Study. Am J Ophthalmol 2016;168:237-49.

28. Lijmer JG, Mol BW, Heisterkamp S, Bonsel GJ, Prins MH, van der Meulen JH, et al. Empirical evidence of design-related bias in studies of diagnostic tests. JAMA 1999;282:1061-6.

29. Medeiros FA, Ng D, Zangwill LM, Sample PA, Bowd C, Weinreb RN. The effects of study design and spectrum bias on the evaluation of diagnostic accuracy of confocal scanning laser ophthalmoscopy in glaucoma. Invest Ophthalmol Vis Sci 2007;48:214-22.

30. Rao HL, Kumbar T, Addepalli UK, Bharti N, Senthil S, Choudhari NS, et al. Effect of spectrum bias on the diagnostic accuracy of spectral-domain optical coherence tomography in glaucoma. Invest Ophthalmol Vis Sci 2012;53:1058-65. 


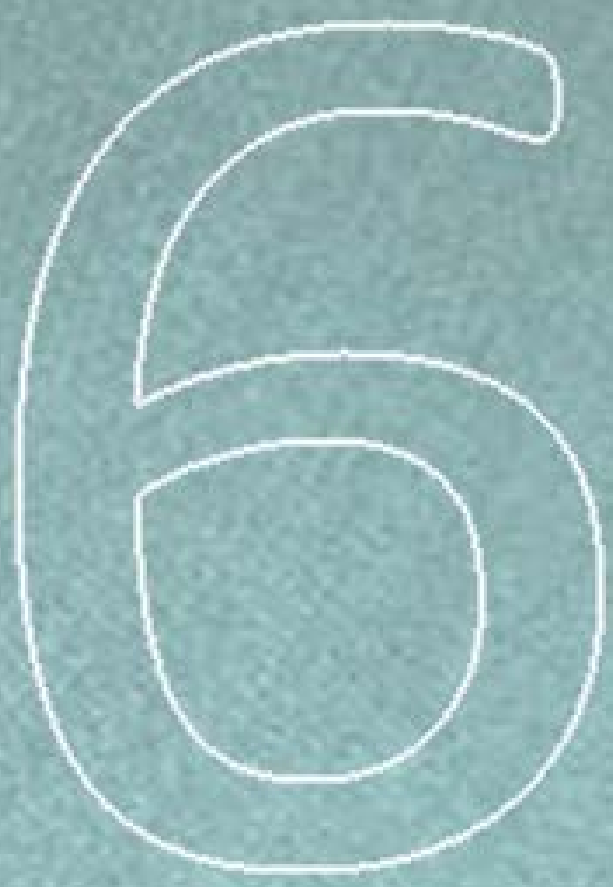

\section{Peripapillary vessel density of OCT angiography in POAG and PACG}

Diagnostic ability of peripapillary vessel density measurements of optical coherence tomography angiography in primary open-angle and angle-closure glaucoma

Harsha L Rao, Sujatha V Kadambi, Robert N Weinreb, Narendra K Puttaiah, Zia S Pradhan, Dhanaraj A S Rao, Rajesh S Kumar, Carroll AB Webers, Rohit Shetty.

Br J Ophthalmol 2017 Aug;101(8):1066-1070. 


\section{ABSTRACT}

Aims: To evaluate the diagnostic ability of peripapillary vessel density measurements on optical coherence tomography angiography (OCTA) in primary open-angle (POAG) and primary angle-closure glaucoma (PACG), and to compare these with peripapillary retinal nerve fiber layer (RNFL) thickness measurements.

Methods: In a cross-sectional study, 48 eyes of 33 healthy control subjects, 63 eyes of 39 POAG patients and 49 eyes of 32 PACG patients underwent OCTA (RTVue-XR, Optovue Inc., Fremont, CA) and RNFL imaging with spectral domain OCT. Diagnostic abilities of vessel density and RNFL parameters were evaluated using area under receiver operating characteristic curves (AUC) and sensitivities at fixed specificities.

Results: AUCs of peripapillary vessel density ranged between 0.48 for the temporal sector and 0.88 for inferotemporal sector in POAG. The same in PACG ranged between 0.57 and 0.86 . Sensitivities at $95 \%$ specificity ranged from $13 \%$ to $70 \%$ in POAG, and from $10 \%$ to $67 \%$ in PACG. AUCs of peripapillary RNFL thickness ranged between 0.51 for the temporal and 0.91 for inferonasal sector in POAG. The same in PACG ranged between 0.61 and 0.87 . Sensitivities at $95 \%$ specificity ranged from $8 \%$ to $68 \%$ in POAG, and $2 \%$ to $67 \%$ in PACG. AUCs of all peripapillary vessel density measurements were comparable $(p>0.05)$ to the corresponding RNFL thickness measurements in both POAG and PACG.

Conclusions: Diagnostic ability of peripapillary vessel density parameters of OCTA, especially the inferotemporal sector measurement, was good in POAG and PACG. Diagnostic abilities of vessel density measurements were comparable to RNFL measurements in both POAG and PACG. 


\section{INTRODUCTION}

Glaucoma is a chronic progressive optic neuropathy with characteristic optic disc and retinal nerve fiber layer (RNFL) changes. Although the exact pathogenic mechanisms of glaucoma are not fully understood, intraocular pressure (IOP) is a major causal factor with the risk of incident glaucoma and its progression increasing with higher IOP. ${ }^{1}$ It also has been proposed that reduced optic nerve head $(\mathrm{ONH})$ perfusion is a cause of glaucoma in at least some individuals. ${ }^{2}, 3$ Earlier studies have measured $\mathrm{ONH}$ blood flow using a variety of techniques and have shown reduction in $\mathrm{ONH}$ perfusion in patients with glaucoma. However, each of these techniques have certain limitations. ${ }^{4}$

Recently, optical coherence tomography (OCT) has been used to develop a threedimensional angiography algorithm called split spectrum amplitude-decorrelation angiography (SSADA) for imaging the ONH microcirculation. ${ }^{5}$ Studies with this OCT angiography (OCTA) have demonstrated reduced $\mathrm{ONH}^{6-9}$ and peripapillary ${ }^{10-}$ 12 vessel density in patients with glaucoma. Most of the previous studies included patients with primary open-angle glaucoma (POAG) and there are no reports till date on the utility of peripapillary vessel density parameters of OCTA in eyes with primary angle-closure glaucoma (PACG). The purpose of this study was to evaluate the diagnostic ability of the peripapillary vessel density in different sectors on OCTA in POAG and PACG, and to compare these with peripapillary RNFL thickness as measured by spectral domain OCT (SD-OCT).

\section{METHODS}

This was a cross-sectional study conducted at a tertiary eye care center between June 2015 and August 2015. The methodology adhered to the tenets of the Declaration of Helsinki. Written informed consent was obtained from all participants and the study was approved by the Institute's Ethics Committee.

Participants of the study included POAG patients, PACG patients and a group of 
control subjects. Control eyes in the study had IOP $\leq 21 \mathrm{~mm} \mathrm{Hg}$, no family history of glaucoma, normal anterior and posterior segment on clinical examination and non-glaucomatous optic discs, as assessed by experts on disc photographs. Both PACG and POAG patients had optic nerve head changes characteristic of glaucoma (focal or diffuse neuroretinal rim thinning, localized notching or RNFL defects). PACG patients had occludable anterior chamber angles in 3 or more quadrants, with goniosynechiae and IOP $21 \mathrm{~mm} \mathrm{Hg}$ at the time of diagnosis. Anterior chamber angle was examined using an indentation gonioscope and was considered occludable if the posterior trabecular meshwork was not seen in the primary position. POAG patients had open angles and IOP> $21 \mathrm{~mm} \mathrm{Hg}$ at the time of diagnosis. Inclusion criteria for all participants were age $\geq 18$ years, corrected distance visual acuity (CDVA) of $20 / 40$ or better and refractive error within $\pm 5 D$ sphere and $\pm 3 \mathrm{D}$ cylinder. Exclusion criteria were presence of any media opacities, retinal or neurological disease that could confound the examinations. Eyes with history of trauma or inflammation were also excluded. All participants underwent a detailed medical history, CDVA measurement, slit-lamp biomicroscopy, Goldmann applanation tonometry, gonioscopy, dilated fundus examination, visual field (VF) examination, stereoscopic optic disc photography, OCTA imaging with RTVue-XR SDOCT (Optovue Inc., Fremont, CA) and RNFL imaging with Cirrus HD-OCT (Carl Zeiss Meditec Inc, Dublin, CA). All examinations were performed on the same day.

\section{Visual field testing}

VF examination was performed using a Humphrey Field analyzer II, model 720i (Zeiss Humphrey Systems, Dublin, CA), with the Swedish interactive threshold algorithm (SITA) standard 24-2 program. VFs were considered reliable if the fixation losses, false positive and false negative response rates were all less than or equal to $20 \%$. 


\section{Optic disc photography}

Stereoscopic optic disc photographs were obtained by trained technicians using a digital fundus camera (Kowa nonmyd WX, Kowa Company, Ltd., Japan). Each optic disc photograph was evaluated independently by two of the three experts (HLR, ZSP and NKP) in a masked manner to determine the presence of glaucomatous changes. Discrepancies between any two experts were adjudicated by the third expert.

\section{OCTA examination}

OCTA imaging was performed using RTVue-XR SDOCT (AngioVue, v2015.100.0.33) after pupillary dilatation. RTVue-XR scans the optic disc using an $840 \mathrm{~nm}$ diode laser source, with an A-scan rate of $70 \mathrm{kHz}$ per second. Optic disc imaging is performed using a set of 2 scans; one vertical priority and one horizontal priority raster volumetric scan covering $4.5 \times 4.5 \mathrm{~mm}$. An orthogonal registration algorithm is used to produce merged 3-dimensional OCT angiograms. ${ }^{13}$ The SSADA algorithm compares the consecutive B-scans at the same location to detect flow using motion contrast. ${ }^{5}$ The software automatically fits an ellipse to the optic disc margin. The peripapillary region is defined as a $0.75 \mathrm{~mm}$-wide elliptical annulus extending from the optic disc boundary (Figure 1). An en face angiogram of the circulation is obtained by the maximum flow (decorrelation value) projection from the inner limiting membrane (ILM) to retinal pigment epithelium (RPE). In this study, the peripapillary vessels were analyzed in the Radial Peripapillary Capillary (RPC) zone. RPC zone extends from the ILM to the nerve fiber layer. Peripapillary vessel density was defined as the percentage area occupied by the large vessels and microvasculature in peripapillary region. The peripapillary region was divided into 6 sectors based on the Garway-Heath map (Figure 1). ${ }^{14}$ Poor quality images with a signal strength index (SSI) less than 40 or images with residual motion artifacts were excluded from the analysis. 


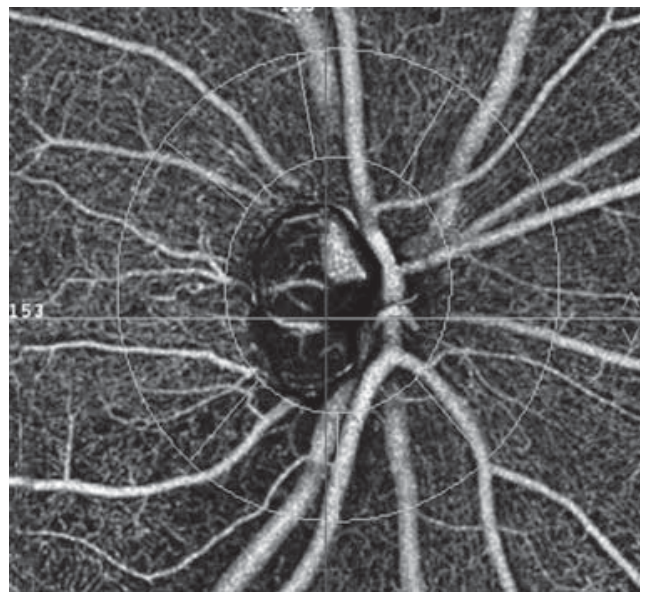

Figure 1. Figure showing the peripapillary sectors where vessel densities are calculated. The vessel density is calculated over a $0.75 \mathrm{~mm}$-wide elliptical annulus extending from the optic disc boundary.

\section{RNFL imaging}

RNFL thickness measurements were performed with Cirrus HD-OCT (software version 7.0.1.290) using the Optic Disc Cube $200 \times 200$ protocol. $^{15,16}$ The 12 clockhour RNFL thickness measurements (in the right eye format) were grouped to closely match the sectors of the OCTA parameters. Clock-hours 8, 9 and 10 were considered as the temporal sector, 11 as the superotemporal sector, 12 and 1 as superonasal, 2, 3 and 4 as nasal, 5 and 6 as inferonasal and 7 as inferotemporal sector. Only good quality scans with signal strength $\geq 6$, absence of motion and blinking artifacts, and absence of segmentation failure were used for the analysis.

\section{STATISTICAL ANALYSIS}

Receiver operating characteristic (ROC) curves and sensitivities at fixed specificities were used to describe the ability of OCTA and RNFL parameters to discriminate 
glaucomatous eyes from control eyes. To obtain confidence intervals for area under the ROC curves (AUC) and sensitivities, a bootstrap re-sampling procedure was used ( $n=1000$ re-samples). As measurements from both eyes of the same subject are likely to be correlated, the cluster of data for the study subject was considered as the unit of resampling and bias corrected standard errors were calculated. This procedure has been used to adjust for the presence of multiple correlated measurements from the same unit. ${ }^{17,18}$ ROC regression modeling technique was used to evaluate the effect of glaucoma severity on the sensitivities of OCTA and RNFL parameters in diagnosing glaucoma. ${ }^{19,} 20$

Statistical analyses were performed using commercial software (Stata ver. 12.1; StataCorp, College Station, TX). A p value of $\leq 0.05$ was considered statistically significant.

\section{RESULTS}

Two hundred and twenty eyes of 139 subjects ( 62 eyes of 43 normal, 90 eyes of 53 POAG and 68 eyes of 43 PACG subjects) underwent OCTA imaging with SD-OCT. Among these, 60 eyes were excluded due to poor quality OCTA scans (27 eyes), RNFL scans ( 5 eyes), unreliable VF (19 eyes) or combinations of these (9 eyes), leaving 160 eyes ( 48 eyes of 33 control subjects, 63 eyes of 39 POAG and 49 eyes of 32 PACG patients) for the analysis. The pairwise agreement between the three experts for optic disc classification on stereo photographs ranged between $90.5 \%$ and $94.9 \%$. Kappa ranged between 0.80 and 0.92 . Twelve eyes each in the POAG and PACG group had normal VFs (PSD with $p>5 \%$ and/or within normal limits or borderline GHT result). Table 1 shows the characteristics of these 3 groups. POAG and PACG patients were significantly older than control subjects. SSI of OCTA scans and signal strength of RNFL scans were significantly greater in the control compared to POAG and PACG subjects. AUCs and sensitivities at fixed specificities were therefore calculated after adjusting for the difference in age and signal strength between the groups using covariate-adjustment as proposed by Pepe. ${ }^{21}$ All vessel density and RNFL measurements were significantly lesser 
in the glaucoma group. Though the median MD was worse in PACG compared to POAG eyes, the difference was not statistically significant $(p=0.49)$.

Table 1. Demographic, visual field, vessel density and retinal nerve fiber layer thickness (RNFL) characteristics of the participants.

\begin{tabular}{|c|c|c|c|c|c|}
\hline & $\begin{array}{c}\text { Control subjects } \\
\text { (48 eyes, } 33 \\
\text { subjects) }\end{array}$ & $\begin{array}{c}\text { POAG group } \\
\text { (63 eyes, } 39 \\
\text { subjects) } \\
\end{array}$ & $\begin{array}{c}\text { PACG group } \\
\text { (49 eyes, } 32 \\
\text { subjects) } \\
\end{array}$ & $\mathbf{P}^{1}$ & $\mathbf{P}^{2}$ \\
\hline Age (years) & $\begin{array}{c}52 \\
(45,62)\end{array}$ & $\begin{array}{c}65 \\
(59,70)\end{array}$ & $\begin{array}{c}64 \\
(59,68)\end{array}$ & $<0.001$ & 0.002 \\
\hline Gender (male:female) & $16: 17$ & $25: 14$ & $14: 18$ & 0.18 & 0.70 \\
\hline Sphere (D) & $\begin{array}{c}0.5 \\
(0,1.5)\end{array}$ & $\begin{array}{c}0 \\
(0,1)\end{array}$ & $\begin{array}{c}0.75 \\
(0,1.5)\end{array}$ & 0.26 & 0.59 \\
\hline Cylinder (D) & $\begin{array}{c}-0.5 \\
(-0.75,0)\end{array}$ & $\begin{array}{c}-0.75 \\
(-1.25,0)\end{array}$ & $\begin{array}{c}-0.75 \\
(-1.25,0)\end{array}$ & 0.01 & 0.10 \\
\hline Intraocular pressure $(\mathrm{mm} \mathrm{Hg})$ & $\begin{array}{c}14 \\
(13,17)\end{array}$ & $\begin{array}{c}16 \\
(12,18)\end{array}$ & $\begin{array}{c}15 \\
(13,18)\end{array}$ & 0.57 & 0.37 \\
\hline Lens (phakia:pseudophakia) & $46: 2$ & $45: 18$ & $44: 5$ & 0.001 & 0.26 \\
\hline Hypertension (yes:no) & $10: 23$ & $10: 29$ & $13: 19$ & 0.66 & 0.38 \\
\hline Diabetes mellitus (yes:no) & $8: 25$ & $15: 24$ & $12: 20$ & 0.20 & 0.25 \\
\hline Mean deviation $(\mathrm{dB})$ & $\begin{array}{c}-1.5 \\
(-2.8,-0.3)\end{array}$ & $\begin{array}{c}-6.3 \\
(-13.5,-3.1)\end{array}$ & $\begin{array}{c}-9.2 \\
(-16.0,-3.3)\end{array}$ & $<0.001$ & $<0.001$ \\
\hline Pattern standard deviation $(\mathrm{dB})$ & $\begin{array}{c}1.9 \\
(1.6,2.2)\end{array}$ & $\begin{array}{c}6.2 \\
(3.0,9.8)\end{array}$ & $\begin{array}{c}7.3 \\
(2.7,11.5)\end{array}$ & $<0.001$ & $<0.001$ \\
\hline Visual field index (\%) & $\begin{array}{c}99 \\
(97,99)\end{array}$ & $\begin{array}{c}87 \\
(64,95)\end{array}$ & $\begin{array}{c}88 \\
(61,97)\end{array}$ & $<0.001$ & $<0.001$ \\
\hline Signal strength index (OCTA) & $\begin{array}{c}61 \\
(53,66)\end{array}$ & $\begin{array}{c}53 \\
(47,60)\end{array}$ & $\begin{array}{c}53 \\
(47,57)\end{array}$ & $<0.001$ & $<0.001$ \\
\hline Average Peripapillary vessel density (\%) & $\begin{array}{c}60.8 \\
(57.2,62.2)\end{array}$ & $\begin{array}{c}52.5 \\
(47.6,57.5)\end{array}$ & $\begin{array}{c}52.7 \\
(48.4,56.7)\end{array}$ & $<0.001$ & $<0.001$ \\
\hline Nasal vessel density (\%) & $\begin{array}{c}58.0 \\
(56.6,61.0)\end{array}$ & $\begin{array}{c}51.2 \\
(47.0,57.0)\end{array}$ & $\begin{array}{c}51.9 \\
(46.4,58.6)\end{array}$ & $<0.001$ & $<0.001$ \\
\hline Inferonasal vessel density (\%) & $\begin{array}{c}62.0 \\
(59.1,64.8)\end{array}$ & $\begin{array}{c}53.8 \\
(46.5,59.4)\end{array}$ & $\begin{array}{c}53.1 \\
(47.4,59.0)\end{array}$ & $<0.001$ & $<0.001$ \\
\hline Inferotemporal vessel density (\%) & $\begin{array}{c}64.6 \\
(62.3,66.6)\end{array}$ & $\begin{array}{c}49.7 \\
(41.9,59.4)\end{array}$ & $\begin{array}{c}52.8 \\
(43.8,59.4)\end{array}$ & $<0.001$ & $<0.001$ \\
\hline Superotemporal vessel density (\%) & $\begin{array}{c}63.3 \\
(59.8,66.5)\end{array}$ & $\begin{array}{c}56.5 \\
(45.0,62.0)\end{array}$ & $\begin{array}{c}53.5 \\
(45.2,59.2)\end{array}$ & $<0.001$ & $<0.001$ \\
\hline Superonasal vessel density (\%) & $\begin{array}{c}59.1 \\
(56.6,62.5)\end{array}$ & $\begin{array}{c}51.8 \\
(46.1,58.3)\end{array}$ & $\begin{array}{c}53.0 \\
(46.7,60.1)\end{array}$ & $<0.001$ & 0.002 \\
\hline Temporal vessel density (\%) & $\begin{array}{c}59.0 \\
(53.9,61.1)\end{array}$ & $\begin{array}{c}55.0 \\
(49.3,59.4)\end{array}$ & $\begin{array}{c}53.6 \\
(47.7,57.9)\end{array}$ & 0.008 & 0.002 \\
\hline Signal strength (ONH cube) & $\begin{array}{c}9 \\
(8,9)\end{array}$ & $\begin{array}{c}8 \\
(8,9)\end{array}$ & $\begin{array}{c}8 \\
(7,9)\end{array}$ & 0.01 & $<0.001$ \\
\hline $\begin{array}{l}\text { Average Peripapillary RNFL thickness } \\
(\mu \mathrm{m})\end{array}$ & $\begin{array}{c}93 \\
(86,99)\end{array}$ & $\begin{array}{c}72 \\
(60,78)\end{array}$ & $\begin{array}{c}70 \\
(64,80)\end{array}$ & $<0.001$ & $<0.001$ \\
\hline
\end{tabular}




\begin{tabular}{|c|c|c|c|c|c|}
\hline Nasal RNFL thickness $(\mu \mathrm{m})$ & $\begin{array}{c}74 \\
(62,81)\end{array}$ & $\begin{array}{c}65 \\
(58,72)\end{array}$ & $\begin{array}{c}65 \\
(60,73)\end{array}$ & $<0.001$ & 0.02 \\
\hline Inferonasal RNFL thickness ( $\mu \mathrm{m})$ & $\begin{array}{c}116 \\
(103,131)\end{array}$ & $\begin{array}{c}79 \\
(62,92)\end{array}$ & $\begin{array}{c}81 \\
(63,100)\end{array}$ & $<0.001$ & $<0.001$ \\
\hline $\begin{array}{l}\text { Inferotemporal RNFL thickness } \\
(\mu \mathrm{m})\end{array}$ & $\begin{array}{c}121 \\
(112,139)\end{array}$ & $\begin{array}{c}66 \\
(55,98)\end{array}$ & $\begin{array}{c}74 \\
(58,94)\end{array}$ & $<0.001$ & $<0.001$ \\
\hline $\begin{array}{l}\text { Superotemporal RNFL thickness } \\
(\mu \mathrm{m})\end{array}$ & $\begin{array}{c}121 \\
(104,135)\end{array}$ & $\begin{array}{c}88 \\
(63,109)\end{array}$ & $\begin{array}{c}81 \\
(56,101)\end{array}$ & $<0.001$ & $<0.001$ \\
\hline Superonasal RNFL thickness $(\mu \mathrm{m})$ & $\begin{array}{c}116 \\
(107,128)\end{array}$ & $\begin{array}{c}83 \\
(69,99)\end{array}$ & $\begin{array}{c}87 \\
(72,101)\end{array}$ & $<0.001$ & $<0.001$ \\
\hline Temporal RNFL thickness $(\mu \mathrm{m})$ & $\begin{array}{c}59 \\
(53,65)\end{array}$ & $\begin{array}{c}54 \\
(46,61)\end{array}$ & $\begin{array}{c}51 \\
(43,59)\end{array}$ & 0.01 & 0.001 \\
\hline
\end{tabular}

$\mathrm{dB}$ : decibel; OCTA: optical coherence tomograph angiography; All values are median with interquartile range in parenthesis. $\mathrm{P}^{1}$ represents the $\mathrm{p}$ value associated with the comparisons between control and primary open angle glaucoma (POAG) groups. $P^{2}$ represents the $p$ value associated with the comparisons between control and primary angle closure glaucoma (PACG) groups.

The AUCs and sensitivities at fixed specificities of the peripapillary vessel density and RNFL parameters are shown in Table 2 and 3 respectively. Inferior sector measurements of both the vessel density and RNFL thickness showed the best AUCs and sensitivities at fixed specificities in POAG and PACG.

Table 2. Diagnostic ability of peripapillary vessel density and retinal nerve fiber layer parameters in differentiating primary open angle glaucoma from control eyes (figures in parenthesis represent $95 \%$ confidence intervals).

\begin{tabular}{lcccccc}
\hline & \multicolumn{3}{c}{ Vessel density } & \multicolumn{3}{c}{ RNFL thickness } \\
\cline { 2 - 6 } & AUC & Sensitivity at & Sensitivity at & \multirow{2}{*}{ AUC } & Sensitivity at & Sensitivity at \\
& & $95 \%$ specificity & $80 \%$ specificity & & $95 \%$ specificity $80 \%$ specificity \\
\hline \multirow{2}{*}{ Average } & 0.79 & $43 \%$ & $67 \%$ & 0.87 & $44 \%$ & $87 \%$ \\
Nasal & $(0.66-0.91)$ & $(18-69)$ & $(41-89)$ & $(0.74-0.94)$ & $(15-73)$ & $(61-98)$ \\
& 0.74 & $46 \%$ & $56 \%$ & 0.69 & $08 \%$ & $40 \%$ \\
Inferonasal & $(0.59-0.86)$ & $(20-65)$ & $(30-73)$ & $(0.51-0.82)$ & $(01-33)$ & $(06-75)$ \\
& 0.77 & $35 \%$ & $59 \%$ & 0.90 & $68 \%$ & $86 \%$ \\
Inferotemporal & $(0.63-0.90)$ & $(13-62)$ & $(34-87)$ & $(0.78-0.97)$ & $(15-91)$ & $(61-96)$ \\
& 0.88 & $70 \%$ & $79 \%$ & 0.87 & $65 \%$ & $83 \%$ \\
Superotemporal & $(0.78-0.94)$ & $(50-83)$ & $(63-91)$ & $(0.78-0.93)$ & $(46-81)$ & $(69-93)$ \\
& 0.68 & $38 \%$ & $44 \%$ & 0.70 & $41 \%$ & $56 \%$ \\
Superonasal & $0.54-0.83)$ & $(23-58)$ & $(28-61)$ & $(0.56-0.82)$ & $(18-61)$ & $(37-74)$ \\
& $(0.54-0.85)$ & $(07-58)$ & $(24-78)$ & $(0.70-0.94)$ & $(06-84)$ & $(49-93)$ \\
Temporal & 0.48 & $13 \%$ & $25 \%$ & 0.51 & $24 \%$ & $35 \%$ \\
& $(0.33-0.67)$ & $(04-41)$ & $(07-48)$ & $(0.37-0.65)$ & $(11-41)$ & $(19-55)$ \\
\hline
\end{tabular}

AUC: area under the receiver operating characteristic curve. 
Table 3. Diagnostic ability of peripapillary vessel density and retinal nerve fiber layer parameters in differentiating primary angle closure glaucoma from control eyes (figures in parenthesis represent $95 \%$ confidence intervals).

\begin{tabular}{lcccccc}
\hline & \multicolumn{3}{c}{ Vessel density } & \multicolumn{3}{c}{ RNFL thickness } \\
\cline { 2 - 7 } & AUC & $\begin{array}{c}\text { Sensitivity } \\
\text { at } 95 \% \\
\text { specificity }\end{array}$ & $\begin{array}{c}\text { Sensitivity } \\
\text { at } 80 \% \\
\text { specificity }\end{array}$ & AUC & $\begin{array}{c}\text { Sensitivity } \\
\text { at } 95 \% \\
\text { specificity }\end{array}$ & $\begin{array}{c}\text { Sensitivity } \\
\text { at } 80 \% \\
\text { specificity }\end{array}$ \\
\hline Average & 0.79 & $43 \%$ & $69 \%$ & 0.86 & $39 \%$ & $82 \%$ \\
& $(0.63-0.93)$ & $(19-74)$ & $(42-93)$ & $(0.73-0.93)$ & $(04-66)$ & $(55-96)$ \\
Nasal & 0.69 & $47 \%$ & $55 \%$ & 0.61 & $02 \%$ & $18 \%$ \\
& $(0.51-0.83)$ & $(26-68)$ & $(29-72)$ & $(0.44-0.70)$ & $(02-04)$ & $(03-38)$ \\
Inferonasal & 0.75 & $37 \%$ & $63 \%$ & 0.83 & $59 \%$ & $76 \%$ \\
& $(0.60-0.89)$ & $(16-66)$ & $(38-92)$ & $(0.70-0.93)$ & $(12-84)$ & $(51-91)$ \\
Inferotemporal & 0.86 & $67 \%$ & $78 \%$ & 0.87 & $67 \%$ & $82 \%$ \\
& $(0.74-0.93)$ & $(38-83)$ & $(59-91)$ & $(0.76-0.94)$ & $(36-80)$ & $(67-93)$ \\
Superotemporal & 0.77 & $51 \%$ & $59 \%$ & 0.78 & $47 \%$ & $63 \%$ \\
& $(0.63-0.89)$ & $(34-73)$ & $(37-76)$ & $(0.64-0.88)$ & $(25-70)$ & $(46-83)$ \\
Superonasal & 0.66 & $18 \%$ & $57 \%$ & 0.80 & $31 \%$ & $71 \%$ \\
& $(0.46-0.81)$ & $(04-56)$ & $(21-77)$ & $(0.65-0.91)$ & $(03-73)$ & $(42-88)$ \\
Temporal & 0.57 & $10 \%$ & $35 \%$ & 0.62 & $33 \%$ & $45 \%$ \\
& $(0.40-0.73)$ & $(02-27)$ & $(09-63)$ & $(0.49-0.75)$ & $(16-54)$ & $(25-63)$ \\
\hline
\end{tabular}

AUC: area under the receiver operating characteristic curve.

AUCs of the OCTA parameters were comparable ( $p>0.05)$ to the corresponding RNFL thickness parameters both in POAG and PACG groups. Sensitivities at fixed specificities of most of the OCTA parameters were also comparable to the corresponding RNFL thickness measurements (overlapping 95\% Cls). AUCs and sensitivities at fixed specificities of OCTA and RNFL thickness measurements in POAG were comparable to that in PACG ( $p>0.05$ for all comparisons).

ROC regression analysis showed a statistically significant influence of glaucoma severity (as measured by mean deviation on VF) on the diagnostic abilities of both peripapillary vessel density and RNFL measurements ( $p<0.05$ for all parameters). Figure 2 shows the effect of glaucoma severity on the sensitivity at $95 \%$ specificity of the inferotemporal peripapillary vessel density and RNFL measurements in (a) POAG and (b) PACG. Sensitivities of both the vessel density and RNFL thickness parameters increased significantly as the severity of glaucoma increased. Sensitivities of the inferotemporal peripapillary vessel density appeared to be better in POAG compared to PACG with increasing severity of the disease. 
(a) POAG

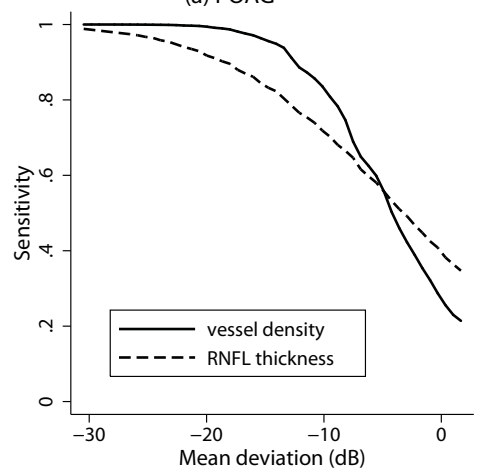

(b) PACG

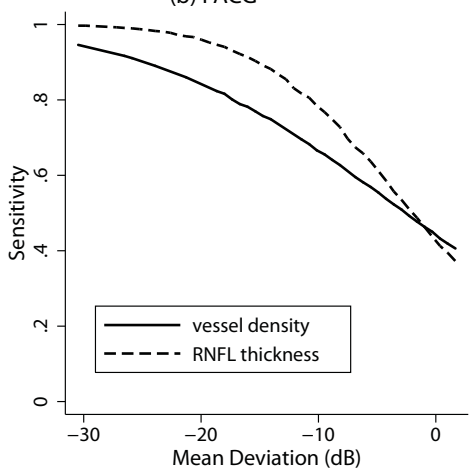

Figure 2. Sensitivity at $95 \%$ specificity of inferotemporal sector vessel density and retinal nerve fiber layer (RNFL) thickness measurements in (a) primary open angle glaucoma and (b) primary angle closure glaucoma.

We ran the entire analysis considering one eye of subjects who contributed both eyes for our earlier analysis and found similar results. When considering the better eye of the glaucoma patients for analysis (median MD: $-5.3 \mathrm{~dB}$ both in POAG and PACG group), inferotemporal sector vessel density showed the best AUC both in POAG (0.87) and PACG (0.86). AUC of inferotemporal RNFL thickness was 0.86 and 0.85 in POAG and PACG respectively. When considering the worse eye of the glaucoma patients for analysis (median MD: -6.8 dB in POAG and -11.1 $\mathrm{dB}$ in PACG), inferotemporal sector vessel density showed the best AUC both in POAG (0.92) and PACG (0.87). AUC of inferotemporal RNFL thickness was 0.89 and 0.88 in POAG and PACG respectively. AUCs of the OCTA parameters were comparable $(p>0.05)$ to the corresponding RNFL thickness parameters both in POAG and PACG groups.

\section{DISCUSSION}

There is limited literature on the diagnostic ability of peripapillary vessel densities of OCTA in POAG ${ }^{10-12}$ and none to our knowledge in PACG. Evaluating 12 glaucoma and 12 normal eyes, Liu et al reported a significant reduction of 
peripapillary vessel density in glaucoma eyes. AUC, sensitivity and specificity of average peripapillary vessel density $(0.94,83.3 \%$ and $91.7 \%$ respectively) was comparable to average RNFL thickness $\left(0.97,91.7 \%\right.$ and $91.7 \%$ respectively). ${ }^{10}$ Yarmohammadi et al compared the diagnostic ability of average peripapillary vessel density and RNFL thickness in 124 eyes with POAG (median MD: -3.9 dB). The AUC of peripapillary vessel density measurement (0.83) was lesser than that of the average RNFL thickness (0.92). This difference however was not statistically significant. ${ }^{11}$ Diagnostic abilities of average vessel density and RNFL thickness in POAG in our study were lesser than that reported by Liu et al but similar to that reported by Yarmohammadi et al. Sector-wise analysis of peripapillary vessel densities was not performed in both these previous studies.

We evaluated the diagnostic ability of peripapillary vessel density measurements in PACG and compared them with the RNFL measurements. We found them to be comparable. We analyzed the diagnostic ability of OCTA parameters separately in POAG and PACG patients because we hypothesized that the role of blood flow may not be the same in the pathogenesis of POAG and PACG, and expected the diagnostic ability of OCTA parameters to be different. However, we found that the AUCs of OCTA measurements in POAG were comparable to that in PACG. The severity of glaucoma in terms of mean deviation on VF, was greater in PACG (-9.2 $\mathrm{dB})$ compared to POAG (-6.3 dB) eyes, in spite of it being statistically comparable. When we accounted for the effect of glaucoma severity on the diagnostic abilities of OCTA parameters, sensitivity of the inferotemporal peripapillary vessel density appeared to be better in POAG compared to PACG with increasing severity of the disease (Figure 2). This may indicate a lower prevalence of ocular perfusion abnormality in PACG compared to POAG. Future studies should evaluate this hypothesis.

Although RTVue-XR provides RNFL thickness measurements in addition to vessel densities, we considered the RNFL thickness parameters from Cirrus HD-OCT for the analysis because Cirrus HD-OCT provides the RNFL thickness in 12 clockhour segments which can be combined into sectors that closely match the vessel density sectors provided by OCTA. Though a previous study has shown significant differences between RTVue and Cirrus OCT in RNFL thickness measurement, ${ }^{22}$ 
a study by Leite et al has shown no difference in their diagnostic abilities in glaucoma. 23

There are a few limitations of the study. Glaucoma patients were significantly older than control subjects. Though we accounted for this difference during the calculation of diagnostic accuracies, it would have been ideal to match the groups during recruitment. The vessel density measurements evaluated in this study were the ones provided by the software automatically. We therefore could not exactly match the vessel density and the RNFL sectors for comparison. This may have affected our results and could also be a reason for the diagnostic ability of the nasal RNFL sectors to be higher than the temporal sectors. The software in its current form does not differentiate the changes in capillaries from that in large vessels. Another possible limitation of the current study was that we did not measure the blood pressure of the subjects or record their anti-hypertensive medication. However, previous studies have shown no relationship between blood pressure readings and peripapillary vessel densities on OCTA. ${ }^{10}$ Lastly, the sample size of our study, though was much larger than the previous studies on OCTA, was still small, as was evidenced by the wide Cls for the AUCs and sensitivities at fixed specificities.

Our study provides directions to future research with OCTA in glaucoma. Our study demonstrated that OCTA has the potential to provide useful information in glaucoma. Though the diagnostic abilities of vessel densities were not better than the tradition RNFL measurements, combining the information from vessel density measurements might enhance the diagnostic yield of other standard tests in glaucoma. Future studies should evaluate this. Being a cross-sectional study, we were unable to evaluate if vascular changes occur before structural (RNFL) changes in the development of glaucoma. Future studies should longitudinally evaluate if vascular changes on OCTA occur earlier than structural or functional changes in glaucoma. Future studies should also evaluate the utility of this new modality in detecting glaucoma progression.

In conclusion, the diagnostic abilities of peripapillary vessel density parameters of OCTA, especially the inferotemporal sector measurement, were good in POAG 
and PACG. Diagnostic abilities of vessel density measurements were comparable to RNFL thickness measurements both in POAG and PACG.

\section{REFERENCES}

1. Weinreb RN, Aung T, Medeiros FA. The pathophysiology and treatment of glaucoma: a review. JAMA 2014;311:1901-1911.

2. Bonomi L, Marchini G, Marraffa M, et al. Vascular risk factors for primary open angle glaucoma: the Egna-Neumarkt Study. Ophthalmology 2000;107:1287-1293.

3. Leske MC, Heijl A, Hyman L, et al. Predictors of long-term progression in the Early Manifest Glaucoma Trial. Ophthalmology 2007;114:1965-1972.

4. Weinreb RN, Harris A. (ed.). Ocular Blood Flow in Glaucoma, The 6th Consensus Report of the World Glaucoma Association. Amsterdam: Kugler Publications; 2009.

5. Jia Y, Tan O, Tokayer J, et al. Split-spectrum amplitude-decorrelation angiography with optical coherence tomography. Opt Express 2012;20:4710-4725.

6. Jia Y, Morrison JC, Tokayer J, et al. Quantitative OCT angiography of optic nerve head blood flow. Biomed Opt Express 2012;3:3127-3137.

7. Jia $Y$, Wei $E$, Wang $X$, et al. Optical coherence tomography angiography of optic disc perfusion in glaucoma. Ophthalmology 2014;121:1322-1332.

8. Wang $\mathrm{X}$, Jiang $\mathrm{C}$, Ko $\mathrm{T}$, et al. Correlation between optic disc perfusion and glaucomatous severity in patients with open-angle glaucoma: an optical coherence tomography angiography study. Graefes Arch Clin Exp Ophthalmol 2015;253:1557-1564.

9. Leveque PM, Zeboulon P, Brasnu E, et al. Optic Disc Vascularization in Glaucoma: Value of Spectral-Domain Optical Coherence Tomography Angiography. J Ophthalmol 2016;2016:6956717.

10. Liu L, Jia Y, Takusagawa HL, et al. Optical Coherence Tomography Angiography of the Peripapillary Retina in Glaucoma. JAMA Ophthalmol 2015;133:1045-1052.

11. Yarmohammadi A, Zangwill LM, Diniz-Filho A, et al. Optical Coherence Tomography Angiography Vessel Density in Healthy, Glaucoma Suspect, and Glaucoma Eyes. Invest Ophthalmol Vis Sci 2016;57:OCT451-459.

12. Rao HL, Pradhan ZS, Weinreb RN, et al. Regional comparisons of optical coherence tomography angiography vessel density in primary open angle glaucoma. Am J Ophthalmol 2016;171:7583.

13. Kraus MF, Potsaid B, Mayer MA, et al. Motion correction in optical coherence tomography volumes on a per A-scan basis using orthogonal scan patterns. Biomed Opt Express 2012;3:1182-1199.

14. Garway-Heath DF, Poinoosawmy D, Fitzke FW, et al. Mapping the visual field to the optic disc in normal tension glaucoma eyes. Ophthalmology 2000;107:1809-1815.

15. Leite MT, Zangwill LM, Weinreb RN, et al. Effect of disease severity on the performance of 
Cirrus spectral-domain OCT for glaucoma diagnosis. Invest Ophthalmol Vis Sci 2010;51:41044109.

16. Begum VU, Addepalli UK, Yadav RK, et al. Ganglion cell-inner plexiform layer thickness of high definition optical coherence tomography in perimetric and preperimetric glaucoma. Invest Ophthalmol Vis Sci 2014;55:4768-4775.

17. Zhou XH, Obuchowski NA, McClish DK. Analysis of correlated ROC data. In: Zhou XH, Obuchowski NA, McClish DK, eds. Statistical Methods in Diagnostic Medicine. New York:John Wiley \& Sons, Inc.;2002;274-306.

18. Alonzo TA, Pepe MS. Distribution-free ROC analysis using binary regression techniques. Biostatistics 2002;3:421-432.

19. Pepe MS. Three approaches to regression analysis of receiver operating characteristic curves for continuous test results. Biometrics 1998;54:124-135.

20. Pepe MS. An interpretation for the ROC curve and inference using GLM procedures. Biometrics 2000;56:352-359.

21. Pepe MS. The Statistical Evaluation of Medical Tests for Classification and Prediction. Oxford, UK: Oxford University Press; 2003;130-67.

22. Leite MT, Rao HL, Weinreb RN, et al. Agreement among spectral-domain optical coherence tomography instruments for assessing retinal nerve fiber layer thickness. Am J Ophthalmol 2011;151:85-92.

23. Leite MT, Rao HL, Zangwill LM, et al. Comparison of the diagnostic accuracies of the Spectralis, Cirrus, and RTVue optical coherence tomography devices in glaucoma. Ophthalmology 2011;118:1334-1339. 


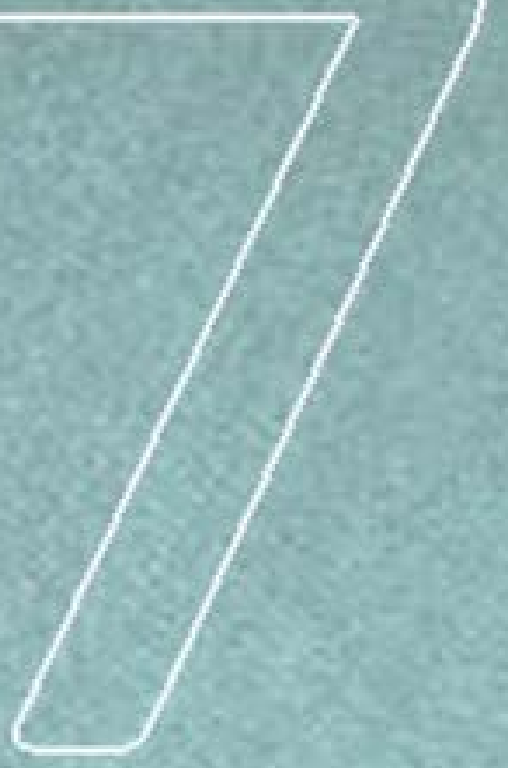

\section{OCT angiography in primary angle closure and glaucoma}

Vessel density and structural measurements of optical coherence tomography in primary angle closure and primary angle closure glaucoma

Harsha L Rao, Zia S Pradhan, Robert N Weinreb, Mohammed Riyazuddin, Srilakshmi Dasari, Jayasree P Venugopal, Narendra K Puttaiah, Dhanaraj A S Rao, Sathi Devi, Kaweh Mansouri, Carroll AB Webers.

Am J Ophthalmol 2017 May;177:106-115 


\section{ABSTRACT}

Purpose: To evaluate the vessel density measurements of the optic nerve head $(\mathrm{ONH})$, peripapillary and macular regions on optical coherence tomography (OCT) angiography in eyes with primary angle closure (PAC) and primary angle closure glaucoma (PACG), and to compare their diagnostic abilities with the ONH rim area, peripapillary retinal nerve fiber layer (RNFL) thickness and the macular ganglion cell complex (GCC) thickness measurements on OCT in PACG.

Design: Cross-sectional study

Methods: Seventy-seven eyes of 50 control subjects, 65 eyes of 45 patients with PACG, and 31 eyes of 22 PAC patients with a history of high IOP, underwent imaging with OCT. Area under receiver operating characteristic curves (AUC) and sensitivities at fixed specificities of vessel densities were compared with structural measurements.

Results: All the vessel density and structural measurements were significantly lower $(p<0.05)$ in the PACG compared to the control group. Vessel densities in the PAC were similar ( $p>0.05$ ) to that of the controls; the superotemporal RNFL, however, was significantly thinner in the PAC group (127 $\mu \mathrm{m}$ vs. $135 \mu \mathrm{m}, \mathrm{p}=0.01)$. The AUC and sensitivity at $95 \%$ specificity of vessel densities within the ONH $(0.76$ \& 42\%) and macular region (0.69 \& 18\%) in PACG were significantly lower $(p<0.001)$ than ONH rim area $(0.90 \& 77 \%)$ and GCC thickness $(0.91 \& 55 \%)$ respectively. AUC and sensitivity of peripapillary vessel density $(0.85 \& 53 \%)$ were similar ( $p=0.25)$ to RNFL thickness (0.91 \& 65\%).

Conclusions: These results suggest that structural changes in PACG occur earlier than the reduction in retinal vessel densities. 


\section{INTRODUCTION}

Optical coherence tomography (OCT) angiography is a relatively new technique of non-invasively imaging the blood vessels of the optic nerve head (ONH) and retina in-vivo. ${ }^{1}$ Studies have used OCT angiography (OCTA) to demonstrate a reduction in vessel density within the $\mathrm{ONH}$, the peripapillary retina and the macula in patients with primary open angle glaucoma (POAG) ${ }^{2-8}$ Additionally, we have also shown that the diagnostic ability of vessel density in the $\mathrm{ONH}$ region was significantly greater in POAG eyes with higher pre-treatment intraocular pressures (IOP). ${ }^{8}$

However, unlike POAG, there is limited literature on the vessel densities with OCTA in primary angle closure disease (PACD). A recent systematic review and meta-analysis of multiple population-based studies has reported that PACD is a significant problem in South-east Asian countries. ${ }^{9}$ PACD includes eyes with primary angle closure (PAC) and primary angle closure glaucoma (PACG). ${ }^{10}$ We hypothesized that the vessel density reduction in PACG, especially the ONH vessel densities, may be greater than that reported in POAG, as PACG presents with higher IOP than POAG. PAC eyes with a history of high IOP and normal optic nerves and visual fields (VF) also provide a unique opportunity to evaluate the effect of high IOP on the vessel densities. This is likely to inform whether vessel density loss is the cause or consequence of neural structure loss. The purpose of the current study was to evaluate the vessel density measurements of the $\mathrm{ONH}$, peripapillary and macular regions in eyes with PACD (PACG eyes and PAC eyes with history of high IOP) using OCTA. In addition, the diagnostic abilities of the vessel density measurements of the $\mathrm{ONH}$, peripapillary and macular regions in PACD were compared with that of the $\mathrm{ONH}$ rim area, peripapillary retinal nerve fiber layer (RNFL) thickness and the macular ganglion cell complex (GCC) thickness measurements on OCT. 


\section{METHODS}

This was a prospective, cross-sectional study conducted at Narayana Nethralaya, a tertiary eye care center in Bengaluru, South India between September 2015 and September 2016. The methodology adhered to the tenets of the Declaration of Helsinki for research involving human subjects. Written informed consent was obtained from all participants and the study was approved by the Institute's Ethics Committee.

Participants of the study included control subjects and PACD patients. Control subjects were either hospital staff or subjects who consulted for a routine eye examination or a refractive error. Control subjects had no family history of glaucoma, IOP $\leq 21 \mathrm{~mm} \mathrm{Hg}$, normal anterior and posterior segments on clinical examination by an ophthalmologist and non-glaucomatous optic discs, as assessed by glaucoma experts on masked examination of stereoscopic optic disc photographs. PACD patients had occludable anterior chamber angles on gonioscopy (before iridotomy), presence of goniosynechiae, a history of IOP >21 $\mathrm{mm} \mathrm{Hg}$, and had undergone laser peripheral iridotomy prior to the OCT imaging. The anterior chamber angle was examined using an indentation gonioscope and was considered occludable if, in primary position, the posterior trabecular meshwork was not seen in 3 or more quadrants. ${ }^{10}$ PACD eyes were divided into PAC and PACG groups depending on the optic nerve head appearance. PACG eyes had glaucomatous changes on optic nerve head examination (focal or diffuse neuroretinal rim thinning, localized notching or RNFL defects) as graded by experts on stereoscopic optic disc photographs. PAC eyes had no evidence of glaucomatous changes on optic disc photographic evaluation. ${ }^{10}$ Inclusion criteria for all participants were age $\geq 18$ years, corrected distance visual acuity of $20 / 40$ or better and refractive error within $\pm 5 \mathrm{D}$ sphere and $\pm 3 \mathrm{D}$ cylinder. Exclusion criteria were presence of any media opacities that prevented good quality OCT scans, or any retinal or neurological disease other than glaucoma, which could confound the evaluation. Eyes with a history of trauma or inflammation were also excluded. All participants underwent a comprehensive ocular examination, which included a detailed medical history, corrected distance visual acuity measurement, slit-lamp 
biomicroscopy, Goldmann applanation tonometry, gonioscopy, dilated fundus examination, stereoscopic disc photography, VF examination and OCT imaging with RTVue-XR SD-OCT (Optovue Inc., Fremont, CA). In addition to IOP measured on the day of scanning, the pre-treatment IOP (i.e. the highest IOP noted before initiating treatment) was documented for all PAC and PACG eyes.

Stereoscopic optic disc photographs were obtained by trained technicians using a digital fundus camera (Kowa nonmyd WX, Kowa Company, Ltd., Japan). Each optic disc photograph was evaluated independently by two glaucoma experts (HLR and NKP) in a masked manner to determine the presence of glaucomatous changes (focal or diffuse neuroretinal rim thinning, localized notching or RNFL defects). The experts were masked to all the clinical data, visual field data and the fellow eye data. Discrepancy in the classification between the two experts was adjudicated by a third glaucoma expert (ZSP).

VF examination was performed using a Humphrey Field analyzer II, model 720i (Zeiss Humphrey Systems, Dublin, CA), with the Swedish interactive threshold algorithm (SITA) standard 24-2 program. VFs were considered reliable if the fixation losses were less than $20 \%$, and the false positive and false negative response rates were less than $15 \%$. VF findings were not considered for defining glaucoma or controls in the primary analysis, but were used for the grading of glaucoma severity.

OCTA imaging of the optic disc region and macula was performed using RTVueXR SD-OCT (AngioVue, v2015.100.0.33). The procedure of OCTA imaging with RTVue-XR has been detailed previously. ${ }^{8}$ In brief, it uses an $840 \mathrm{~nm}$ diode laser source, with an A-scan rate of $70 \mathrm{kHz}$ per second. Imaging is performed using a set of 2 scans; one vertical priority and one horizontal priority raster volumetric scan. The optic disc scan covers an area of $4.5 \times 4.5 \mathrm{~mm}$ and the macular scan was performed using volumetric scans covering $3 \times 3 \mathrm{~mm}$. An orthogonal registration algorithm is used to produce merged 3-dimensional OCT angiograms. ${ }^{11}$ The software compares the consecutive B-scans at the same location to detect flow using motion contrast, thereby delineating blood vessels. ${ }^{1}$ Vessel density is defined as the percentage area occupied by the large vessels and microvasculature in a 
particular region. Vessel densities are calculated over the entire scan area, i.e. whole enface disc and whole enface macula, as well as defined areas within each scan as described below. In addition, the software calculates vessel densities in various layers of the retina and the $\mathrm{ONH}$.

In the optic disc scan, the software automatically fits an ellipse to the optic disc margin and calculates the average vessel density within the ONH (referred to as the inside disc vessel density). The $\mathrm{ONH}$ vessel densities are calculated from the "nerve head segment" of the $\mathrm{ONH}$ angiogram. This segment extends from 2000 microns above the internal limiting membrane (ILM) to 150 microns below the ILM. The ONH is divided into 6 sectors based on the Garway-Heath map and the vessel density in each sector is calculated (nasal, inferonasal, inferotemporal, superotemporal, superonasal and temporal sectors). ${ }^{12}$ In order to compare the $\mathrm{ONH}$ vessel densities with the rim area of $\mathrm{OCT}$, the superonasal and superotemporal sectors were combined together as the superior sector, and the inferonasal and inferotemporal sectors as the inferior sector. In the same optic disc scan, the peripapillary region is defined as a $0.75 \mathrm{~mm}$-wide elliptical annulus extending from the optic disc boundary and the average vessel density within this region is calculated. The peripapillary vessel density was analyzed from the "Radial Peripapillary Capillary (RPC) segment" which extends from the ILM to the posterior boundary of the nerve fiber layer. The peripapillary region is divided into 8 sectors, each of 45 degrees, and peripapillary vessel density in each sector is reported. The 8 sector measurements of peripapillary vessel density were compared with the 8 sector measurements of peripapillary RNFL thickness. Macular vessel densities were analyzed over a $1.5 \mathrm{~mm}$-wide parafoveal, circular annulus centered on the macula. Macular vessel densities analyzed in this study were of the superficial vascular plexus present in the inner layers of the retina (extending from the ILM to the inner plexiform layer). The parafoveal region was divided into 2 sectors of $180^{\circ}$ each (superior and inferior sectors) corresponding to the superior and inferior sector GCC measurements of OCT.

All subjects also underwent the traditional $\mathrm{ONH}$, peripapillary RNFL and macular GCC thickness measurements on RTVue-XR SD-OCT using the ONH and the GCC scans. These scan protocols have been explained in detail previously. ${ }^{13,14}$ 
In brief, the ONH protocol consists of 12 radial scans $3.4 \mathrm{~mm}$ in length, and 6 concentric ring scans ranging from 2.5 to $4.0 \mathrm{~mm}$ in diameter all centered on the optic disc. Retinal pigment epithelium (RPE) tips were automatically detected by the software, which were refined manually by the operator, if needed. The software delineates the optic disc margin by joining the RPE tips. The optic cup is automatically defined by the software by fitting a plane 150 microns parallel to and above a plane that fits the coordinates of the RPE tips. ONH protocol calculates various parameters that describe the $\mathrm{ONH}$ and also generates a polar RNFL thickness map which is the RNFL thickness measured along a circle $3.45 \mathrm{~mm}$ in diameter centered on the optic disc. The GCC scan consists of one horizontal line scan $7 \mathrm{~mm}$ in length and 15 vertical line scans $7 \mathrm{~mm}$ in length and at $0.5 \mathrm{~mm}$ interval centered $1 \mathrm{~mm}$ temporal to the fovea. GCC scan measures the inner retinal thickness which includes the thickness of the nerve fiber layer, ganglion cell layer and the inner plexiform layer, collectively called the GCC thickness.

All the examinations for a particular subject were performed on the same day. Image quality was assessed for all OCTA and OCT scans. Poor quality images, which were defined as those with a signal strength index (SSI) less than 35 or images with motion artifacts and segmentation errors were excluded from the analysis.

\section{STATISTICAL ANALYSIS}

Descriptive statistics included mean and standard deviation for normally distributed variables and median and inter-quartile range (IQR) for non-normally distributed variables. Shapiro-Wilk test was used to test for the normality distribution of continuous variables. Receiver operating characteristic (ROC) curves were used to describe the ability of vessel density and structural measurements of OCT to discriminate PACG eyes from control eyes. Sensitivities at fixed specificities of $80 \%$ and $95 \%$ were determined for all the parameters. To obtain confidence intervals for area under the ROC curves (AUC) and sensitivities, a bootstrap re-sampling procedure was used ( $n=1000$ re-samples). As measurements from both eyes 
of the same subject are likely to be correlated, the standard statistical methods for parameter estimation can lead to underestimation of standard errors and to confidence intervals that are too narrow. ${ }^{15}$ Therefore, the cluster of data for the study subject was considered as the unit of resampling and bias corrected standard errors were calculated during all estimations. This procedure has been used to adjust for the presence of multiple correlated measurements from the same unit. ${ }^{16,17}$ ROC regression modeling technique was used to evaluate the effect of glaucoma severity, as based on the mean deviation (MD) on VF, on the AUCs and sensitivities of OCT measurements in diagnosing glaucoma. ${ }^{18,19}$

Statistical analyses were performed using commercial software (Stata ver. 13.1; StataCorp, College Station, TX). A $p$ value of $\leq 0.05$ was considered statistically significant.

\section{RESULTS}

One hundred and eighty-five eyes of 124 subjects underwent vessel density and structural imaging with OCT. Eight eyes in the PACD group, in which the optic disc could not be graded as either glaucomatous or normal by the glaucoma experts on stereoscopic photographs (and were graded as "suspect"), were excluded. For the remaining 177 eyes ( 78 eyes of 50 normal, 67 eyes of 46 PACG and 32 eyes of 23 PAC subjects), the image quality of all OCT and OCTA scans was assessed and the number of poor quality scans is shown in Figure 1. Four eyes with poor quality of both OCT and OCTA scans were excluded from the analysis. Final analysis included vessel density and structural scans from 173 eyes of 117 subjects (77 eyes of 50 normal, 65 eyes of 45 PACG and 31 eyes of 22 PAC subjects). Of the 65 eyes with PACG, 14 eyes had a "within normal limit" or a "borderline" glaucoma hemifield test (GHT) result, and / or the probability value of pattern standard deviation (PSD) $>5 \%$ on VF (preperimetric glaucoma). Of the 31 eyes with PAC, 5 had an "outside normal limit" GHT result and a probability of PSD $\leq 5 \%$. Of the 65 eyes with PACG, 14 eyes were not on any anti-glaucoma medications (as they had undergone trabeculectomy), 31 eyes were on topical beta blockers, 25 on alpha 
agonists, 24 on carbonic anhydrase inhibitors and 26 on prostaglandin analogues (either as a monotherapy or as components of the combination therapy). Of the 31 eyes with PAC, 8 eyes were not on any anti-glaucoma medications, 13 eyes were on topical beta blockers, 9 on alpha agonists, 4 on carbonic anhydrase inhibitors and 11 on prostaglandin analogues (either as a monotherapy or as components of the combination therapy). Table 1 shows the clinical, VF, vessel density and structural measurements of the included subjects. All the vessel density and structural measurements in the PACG cohort were significantly lower than the control group. SSI of the ONH scan of OCT was significantly greater in the control subjects compared to the PACG patients. AUCs and sensitivities at fixed specificities of rim area and peripapillary RNFL thickness parameters were therefore calculated after adjusting for the difference in SSI between the control and PACG groups using covariate-adjustment as proposed by Pepe. ${ }^{20} \mathrm{VF}$ indices and vessel densities in the PAC eyes were statistically similar to that in the control eyes and so were the structural OCT measurements except for the superotemporal RNFL which was significantly thinner in the PAC group.

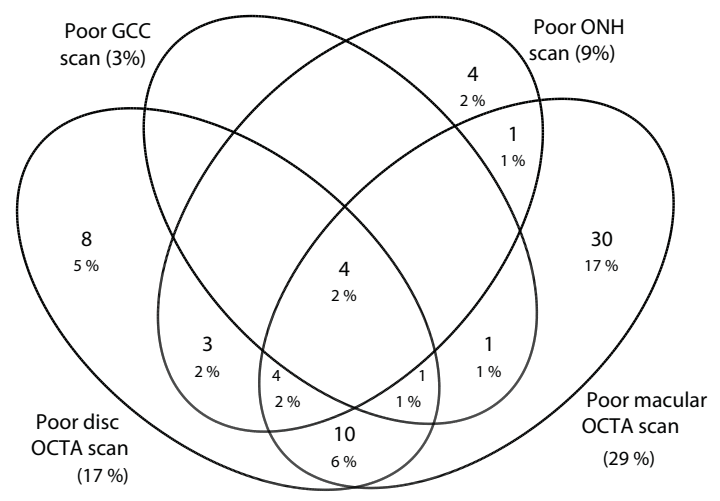

Figure 1. Venn diagram showing the number of poor quality images with the optic disc and macular optical coherence tomography angiography (OCTA) scans and the optic nerve head (ONH) and the ganglion cell complex (GCC) scans. 
Table 1. Clinical features, visual field parameters, vessel density and structural measurements of the participants. All values represent median and interquartile range unless specified.

\begin{tabular}{|c|c|c|c|c|c|}
\hline & $\begin{array}{c}\text { Control } \\
\text { subjects } \\
\text { (77 eyes, } \\
50 \text { subjects) }\end{array}$ & $\begin{array}{c}\text { PAC group } \\
\text { (31 eyes, } \\
22 \text { subjects) }\end{array}$ & $\begin{array}{l}\text { PACG group } \\
\text { (65 eyes, } \\
45 \text { subjects) }\end{array}$ & $\mathrm{P}^{1}$ & $\mathbf{P}^{2}$ \\
\hline Age (years)* & $60.7 \pm 8.3$ & $60.3 \pm 7.9$ & $62.0 \pm 7.8$ & 0.86 & 0.44 \\
\hline Gender (male:female) & $27: 23$ & 7:16 & $25: 21$ & 0.06 & 0.97 \\
\hline Sphere (D) & $\begin{array}{c}0.5 \\
(0,1)\end{array}$ & $\begin{array}{c}1 \\
(0.5,1.75)\end{array}$ & $\begin{array}{c}0.75 \\
(0,1.5)\end{array}$ & 0.08 & 0.43 \\
\hline Cylinder (D) & $\begin{array}{c}-0.5 \\
(-1,-0.5)\end{array}$ & $\begin{array}{c}-0.75 \\
(-0.75,-0.5)\end{array}$ & $\begin{array}{c}-0.75 \\
(-1,-0.5)\end{array}$ & 0.88 & 0.65 \\
\hline Pseudophakia (n, \%) & $13(16.7 \%)$ & $3(9.4 \%)$ & $10(14.9 \%)$ & 0.33 & 0.78 \\
\hline Optic disc area $\left(\mathrm{mm}^{2}\right)$ & $\begin{array}{c}2.28 \\
(1.93,2.53)\end{array}$ & $\begin{array}{c}2.33 \\
(2.11,2.68)\end{array}$ & $\begin{array}{c}2.31 \\
(2.11,2.52)\end{array}$ & 0.18 & 0.50 \\
\hline IOP at the scanning visit ( $\mathrm{mm} \mathrm{Hg}$ ) & $\begin{array}{c}15 \\
(14,18)\end{array}$ & $\begin{array}{c}18 \\
(14,20)\end{array}$ & $\begin{array}{c}17 \\
(14,20)\end{array}$ & 0.002 & $<0.001$ \\
\hline Pre-treatment IOP (mm Hg) & & $\begin{array}{c}25 \\
(23,28)\end{array}$ & $\begin{array}{c}24 \\
(22,29)\end{array}$ & & \\
\hline Hypertension (yes:no) & 19:31 & 7:15 & $16: 29$ & 0.53 & 0.74 \\
\hline Diabetes mellitus (yes:no) & $17: 33$ & $6: 16$ & $17: 28$ & 0.50 & 0.76 \\
\hline Mean deviation (dB) & $\begin{array}{c}-0.9 \\
(-3.5,-0.3)\end{array}$ & $\begin{array}{c}-1.9 \\
(-3.6,-0.8)\end{array}$ & $\begin{array}{c}-8.2 \\
(-16.0,-4.0)\end{array}$ & 0.22 & $<0.001$ \\
\hline Pattern standard deviation (dB) & $\begin{array}{c}1.9 \\
(1.5,2.5)\end{array}$ & $\begin{array}{c}2.1 \\
(1.8,2.6)\end{array}$ & $\begin{array}{c}6.1 \\
(3.0,10.3)\end{array}$ & 0.34 & $<0.001$ \\
\hline Visual field index (\%) & $\begin{array}{c}99 \\
(97,99)\end{array}$ & $\begin{array}{c}98 \\
(96,99)\end{array}$ & $\begin{array}{c}86 \\
(56,95)\end{array}$ & 0.26 & $<0.001$ \\
\hline \multicolumn{6}{|c|}{ OCTA parameters } \\
\hline SSI (Optic disc scan)* & $53.6 \pm 8.9$ & $56.3 \pm 6.7$ & $51.8 \pm 7.6$ & 0.19 & 0.23 \\
\hline Whole enface vd (disc scan, \%) & $\begin{array}{c}53.9 \\
(51.3,55.5)\end{array}$ & $\begin{array}{c}54.0 \\
(50.7,55.1)\end{array}$ & $\begin{array}{c}45.1 \\
(39.5,48.8)\end{array}$ & 0.76 & $<0.001$ \\
\hline Inside disc vd (\%) & $\begin{array}{c}48.1 \\
(44.0,50.0)\end{array}$ & $\begin{array}{c}46.5 \\
(43.4,48.0)\end{array}$ & $\begin{array}{c}41.8 \\
(34.8,45.8)\end{array}$ & 0.26 & $<0.001$ \\
\hline Nasal vd (\%) & $\begin{array}{c}48.9 \\
(44.1,52.7)\end{array}$ & $\begin{array}{c}49.6 \\
(42.8,51.5)\end{array}$ & $\begin{array}{c}44.2 \\
(36.4,47.1)\end{array}$ & 0.63 & $<0.001$ \\
\hline Inferior vd (\%) & $\begin{array}{c}48.7 \\
(44.8,53.3)\end{array}$ & $\begin{array}{c}47.3 \\
(43.8,50.5)\end{array}$ & $\begin{array}{c}43.7 \\
(39.1,50.2)\end{array}$ & 0.14 & $<0.001$ \\
\hline Superior vd (\%) & $\begin{array}{c}49.1 \\
(43.4,52.2)\end{array}$ & $\begin{array}{c}49.5 \\
(45.1,53.7)\end{array}$ & $\begin{array}{c}39.7 \\
(31.9,47.9)\end{array}$ & 0.46 & $<0.001$ \\
\hline Temporal vd (\%) & $\begin{array}{c}44.3 \\
(40.4,50.7)\end{array}$ & $\begin{array}{c}40.5 \\
(37.4,48.3)\end{array}$ & $\begin{array}{c}36.9 \\
(29.6,43.1)\end{array}$ & 0.25 & $<0.001$ \\
\hline
\end{tabular}




\begin{tabular}{|c|c|c|c|c|c|}
\hline Average Peripapillary vd (\%) & $\begin{array}{c}61.9 \\
(59.9,64.2)\end{array}$ & $\begin{array}{c}60.0 \\
(57.4,62.9)\end{array}$ & $\begin{array}{c}53.3 \\
(46.2,58.7)\end{array}$ & 0.12 & $<0.001$ \\
\hline Upper temporal vd (\%) & $\begin{array}{c}62.5 \\
(59.8,66.9)\end{array}$ & $\begin{array}{c}62.3 \\
(58.8,63.8)\end{array}$ & $\begin{array}{c}56.6 \\
(49.1,63.5)\end{array}$ & 0.31 & $<0.001$ \\
\hline Superotemporal vd (\%) & $\begin{array}{c}64.9 \\
(60.7,68.0)\end{array}$ & $\begin{array}{c}64.8 \\
(60.0,66.2)\end{array}$ & $\begin{array}{c}56.9 \\
(47.3,62.9)\end{array}$ & 0.33 & $<0.001$ \\
\hline Superonasal vd (\%) & $\begin{array}{c}59.4 \\
(53.9,62.0)\end{array}$ & $\begin{array}{c}57.8 \\
(53.8,60.1)\end{array}$ & $\begin{array}{c}51.8 \\
(44.3,57.4)\end{array}$ & 0.22 & $<0.001$ \\
\hline Upper nasal vd (\%) & $\begin{array}{c}58.0 \\
(54.7,61.7)\end{array}$ & $\begin{array}{c}58.7 \\
(55.8,62.0)\end{array}$ & $\begin{array}{c}51.8 \\
(45.3,58.3)\end{array}$ & 0.57 & $<0.001$ \\
\hline Lower nasal vd (\%) & $\begin{array}{c}56.9 \\
(54.1,61.0)\end{array}$ & $\begin{array}{c}57.4 \\
(54.3,62.7)\end{array}$ & $\begin{array}{c}48.9 \\
(42.1,55.4)\end{array}$ & 0.47 & $<0.001$ \\
\hline Inferonasal vd (\%) & $\begin{array}{c}61.2 \\
(58.8,64.9)\end{array}$ & $\begin{array}{c}59.6 \\
(57.2,64.0)\end{array}$ & $\begin{array}{c}54.3 \\
(47.3,61.0)\end{array}$ & 0.31 & $<0.001$ \\
\hline Inferotemporal vd (\%) & $\begin{array}{c}63.9 \\
(61.8,67.2)\end{array}$ & $\begin{array}{c}63.8 \\
(61.8,67.6)\end{array}$ & $\begin{array}{c}51.8 \\
(43.4,61.0)\end{array}$ & 0.97 & $<0.001$ \\
\hline Lower temporal vd (\%) & $\begin{array}{c}58.1 \\
(53.9,62.1)\end{array}$ & $\begin{array}{c}60.2 \\
(52.2,62.7)\end{array}$ & $\begin{array}{c}55.9 \\
(48.0,60.7)\end{array}$ & 0.85 & $<0.001$ \\
\hline SSI (Macula scan)* & $61.1 \pm 6.7$ & $64.4 \pm 5.4$ & $62.5 \pm 5.7$ & 0.05 & 0.25 \\
\hline $\begin{array}{l}\text { Whole enface vd (macula scan, } \\
\%)^{*}\end{array}$ & $47.5 \pm 3.8$ & $47.1 \pm 3.4$ & $44.1 \pm 4.3$ & 0.69 & $<0.001$ \\
\hline Parafoveal vd (\%)* & $49.5 \pm 3.9$ & $49.3 \pm 3.5$ & $46.6 \pm 4.6$ & 0.86 & $<0.001$ \\
\hline Superior vd (\%)* & $49.2 \pm 4.4$ & $49.7 \pm 3.7$ & $46.6 \pm 5.0$ & 0.68 & 0.006 \\
\hline Inferior vd (\%)* & $49.3 \pm 4.7$ & $48.9 \pm 3.7$ & $46.5 \pm 4.6$ & 0.72 & 0.002 \\
\hline \multicolumn{6}{|c|}{ OCT parameters } \\
\hline SSI (ONH scan)* & $55.2 \pm 8.1$ & $54.2 \pm 9.7$ & $51.8 \pm 8.2$ & 0.59 & 0.02 \\
\hline Neuroretinal rim area $\left(\mathrm{mm}^{2}\right)$ & $\begin{array}{c}1.32 \\
(1.12,1.51)\end{array}$ & $\begin{array}{c}1.40 \\
(1.17,1.56)\end{array}$ & $\begin{array}{c}0.72 \\
(0.55,0.95)\end{array}$ & 0.43 & $<0.001$ \\
\hline Nasal rim area $\left(\mathrm{mm}^{2}\right)$ & $\begin{array}{c}0.40 \\
(0.34,0.44)\end{array}$ & $\begin{array}{c}0.39 \\
(0.32,0.44)\end{array}$ & $\begin{array}{c}0.20 \\
(0.11,0.28)\end{array}$ & 0.92 & $<0.001$ \\
\hline Inferior rim area $\left(\mathrm{mm}^{2}\right)$ & $\begin{array}{c}0.41 \\
(0.33,0.48)\end{array}$ & $\begin{array}{c}0.41 \\
(0.33,0.48)\end{array}$ & $\begin{array}{c}0.18 \\
(0.12,0.31)\end{array}$ & 0.85 & $<0.001$ \\
\hline Superior rim area $\left(\mathrm{mm}^{2}\right)$ & $\begin{array}{c}0.38 \\
(0.32,0.48)\end{array}$ & $\begin{array}{c}0.41 \\
(0.35,0.47)\end{array}$ & $\begin{array}{c}0.23 \\
(0.16,0.29)\end{array}$ & 0.32 & $<0.001$ \\
\hline Temporal rim area $\left(\mathrm{mm}^{2}\right)$ & $\begin{array}{c}0.12 \\
(0.09,0.19)\end{array}$ & $\begin{array}{c}0.16 \\
(0.12,0.21)\end{array}$ & $\begin{array}{c}0.09 \\
(0.05,0.13)\end{array}$ & 0.05 & $<0.001$ \\
\hline $\begin{array}{l}\text { Peripapillary RNFL thickness } \\
(\mu \mathrm{m})^{*}\end{array}$ & $100 \pm 10$ & $98 \pm 8$ & $78 \pm 14$ & 0.29 & $<0.001$ \\
\hline Upper temporal RNFL $(\mu \mathrm{m})^{*}$ & $76 \pm 11$ & $76 \pm 8$ & $66 \pm 15$ & 0.68 & $<0.001$ \\
\hline $\begin{array}{l}\text { Superotemporal RNFL } \\
(\mu \mathrm{m})^{*}\end{array}$ & $135 \pm 14$ & $127 \pm 11$ & $102 \pm 22$ & 0.01 & $<0.001$ \\
\hline
\end{tabular}




\begin{tabular}{|c|c|c|c|c|c|}
\hline Superonasal RNFL $(\mu \mathrm{m})^{*}$ & $112 \pm 22$ & $107 \pm 13$ & $86 \pm 20$ & 0.25 & $<0.001$ \\
\hline Upper nasal RNFL $(\mu \mathrm{m})^{*}$ & $85 \pm 15$ & $85 \pm 14$ & $67 \pm 14$ & 0.85 & $<0.001$ \\
\hline Lower nasal RNFL $(\mu \mathrm{m})^{*}$ & $75 \pm 12$ & $76 \pm 9$ & $63 \pm 12$ & 0.75 & $<0.001$ \\
\hline Inferonasal RNFL $(\mu \mathrm{m})^{*}$ & $117 \pm 19$ & $117 \pm 14$ & $85 \pm 22$ & 0.95 & $<0.001$ \\
\hline Inferotemporal RNFL $(\mu \mathrm{m})^{*}$ & $133 \pm 16$ & $130 \pm 16$ & $93 \pm 25$ & 0.38 & $<0.001$ \\
\hline Lower temporal RNFL $(\mu \mathrm{m})^{*}$ & $67 \pm 10$ & $66 \pm 12$ & $60 \pm 12$ & 0.63 & $<0.001$ \\
\hline SSI (GCC scan)* & $59.1 \pm 9.7$ & $64.3 \pm 9.4$ & $62.0 \pm 8.9$ & 0.01 & 0.07 \\
\hline Average GCC thickness $(\mu \mathrm{m})^{*}$ & $96.5 \pm 9.4$ & $93.8 \pm 7.3$ & $77.9 \pm 11.9$ & 0.16 & $<0.001$ \\
\hline $\begin{array}{l}\text { Superior GCC thickness } \\
(\mu \mathrm{m})^{*}\end{array}$ & $95.7 \pm 8.2$ & $93.5 \pm 7.8$ & $80.2 \pm 13.2$ & 0.21 & $<0.001$ \\
\hline $\begin{array}{l}\text { Inferior GCC thickness } \\
(\mu \mathrm{m})^{*}\end{array}$ & $97.3 \pm 13.4$ & $94.1 \pm 7.3$ & $75.7 \pm 12.9$ & 0.22 & $<0.001$ \\
\hline
\end{tabular}

PAC: primary angle closure; PACG: primary angle closure glaucoma; D: diopter; dB: decibel; IOP: intraocular pressure; SSI: signal strength index; vd: vessel density; ONH: optic nerve head; RNFL: retinal nerve fiber layer; GCC: ganglion cell complex; ${ }^{*}$ mean \pm standard deviation. $\mathrm{P}^{1}$ represents the $p$ value associated with the comparisons between control and PAC groups. $\mathrm{P}^{2}$ represents the $p$ value associated with the comparisons between control and PACG groups.

The AUCs and sensitivities at fixed specificities of the vessel density measurements to differentiate PACG from control eyes are shown in Table 2 . Whole enface vessel density of the disc scan and average peripapillary vessel density showed the best AUC and sensitivity at fixed specificity to diagnose PACG. The AUCs and sensitivities at fixed specificities of the structural measurements to differentiate PACG from control eyes are shown in Table 3. Average and the inferior quadrant measurements of rim area, RNFL and GCC thickness showed the best AUC and sensitivity at fixed specificity to diagnose PACG. Comparing the diagnostic abilities region-wise, ONH rim area showed a statistically significantly better AUC than the inside disc vessel density $(p<0.001)$, and macular GCC thickness showed a statistically significantly better AUC than the macular vessel density $(p<0.001)$. AUC of peripapillary RNFL thickness was similar $(p=0.25)$ to that of the peripapillary vessel density. 
Table 2. Diagnostic ability of vessel density parameters of optical coherence tomography angiography in differentiating primary angle closure glaucoma from control eyes (figures in parenthesis represent $95 \%$ confidence intervals).

\begin{tabular}{lccc}
\hline \multicolumn{1}{c}{ Vessel density } & AUC & $\begin{array}{c}\text { Sensitivity at } \\
95 \% \text { specificity }\end{array}$ & $\begin{array}{c}\text { Sensitivity at 80\% } \\
\text { specificity }\end{array}$ \\
\hline Whole enface vd (disc scan) & $0.91(0.84-0.95)$ & $66 \%(43-83)$ & $79 \%(66-90)$ \\
Inside disc vd & $0.76(0.66-0.85)$ & $42 \%(03-69)$ & $53 \%(34-67)$ \\
$\quad$ Nasal vd & $0.72(0.61-0.81)$ & $11 \%(02-41)$ & $46 \%(28-67)$ \\
$\quad$ Inferior vd & $0.67(0.56-0.78)$ & $25 \%(06-54)$ & $51 \%(32-67)$ \\
$\quad$ Superior vd & $0.74(0.64-0.83)$ & $26 \%(13-47)$ & $58 \%(37-77)$ \\
$\quad$ Temporal vd & $0.75(0.64-0.83)$ & $12 \%(02-44)$ & $58 \%(31-76)$ \\
Average Peripapillary vd & $0.85(0.78-0.92)$ & $53 \%(34-69)$ & $76 \%(55-89)$ \\
$\quad$ Upper temporal vd & $0.72(0.61-0.82)$ & $41 \%(26-57)$ & $55 \%(40-73)$ \\
$\quad$ Superotemporal vd & $0.83(0.74-0.91)$ & $54 \%(35-70)$ & $67 \%(52-82)$ \\
Superonasal vd & $0.78(0.68-0.86)$ & $48 \%(31-65)$ & $57 \%(40-76)$ \\
$\quad$ Upper nasal vd & $0.73(0.62-0.83)$ & $35 \%(17-62)$ & $62 \%(45-78)$ \\
$\quad$ Lower nasal vd & $0.77(0.68-0.86)$ & $47 \%(30-68)$ & $69 \%(54-81)$ \\
Inferonasal vd & $0.78(0.66-0.87)$ & $47 \%(28-61)$ & $59 \%(39-73)$ \\
$\quad$ Inferotemporal vd & $0.84(0.73-0.92)$ & $60 \%(47-78)$ & $76 \%(57-91)$ \\
Lower temporal vd & $0.62(0.51-0.73)$ & $21 \%(06-44)$ & $41 \%(25-59)$ \\
Whole enface vd (macula scan) & $0.73(0.61-0.83)$ & $24 \%(06-45)$ & $53 \%(26-75)$ \\
Parafoveal vd & $0.69(0.57-0.80)$ & $18 \%(02-37)$ & $49 \%(23-73)$ \\
Superior vd & $0.65(0.52-0.76)$ & $18 \%(04-35)$ & $42 \%(14-65)$ \\
Inferior vd & $0.68(0.57-0.78)$ & $13 \%(02-32)$ & $31 \%(12-67)$ \\
\hline
\end{tabular}

AUC: area under the receiver operating characteristic curve; vd: vessel density.

Table 3. Diagnostic ability of structural parameters of optical coherence tomography in differentiating primary angle closure glaucoma from control eyes (figures in parenthesis represent $95 \%$ confidence intervals).

\begin{tabular}{|c|c|c|c|}
\hline Vessel density & AUC & $\begin{array}{c}\text { Sensitivity at } \\
95 \% \text { specificity }\end{array}$ & $\begin{array}{c}\text { Sensitivity at } 80 \% \\
\text { specificity }\end{array}$ \\
\hline Neuroretinal rim area & $0.90(0.83-0.95)$ & $77 \%(57-90)$ & $88 \%(78-97)$ \\
\hline Nasal rim area & $0.86(0.76-0.93)$ & $64 \%(45-79)$ & $77 \%(59-86)$ \\
\hline Inferior rim area & $0.87(0.80-0.93)$ & $61 \%(48-74)$ & $80 \%(62-91)$ \\
\hline Superior rim area & $0.86(0.78-0.92)$ & $53 \%(34-69)$ & $80 \%(65-91)$ \\
\hline Temporal rim area & $0.75(0.65-0.85)$ & $30 \%(11-43)$ & $59 \%(41-80)$ \\
\hline
\end{tabular}




$\begin{array}{clll}\text { Peripapillary RNFL thickness } & 0.91(0.84-0.95) & 65 \%(46-81) & 83 \%(69-92) \\ \text { Upper temporal RNFL } & 0.70(0.58-0.80) & 30 \%(14-45) & 47 \%(23-61) \\ \text { Superotemporal RNFL } & 0.90(0.83-0.95) & 69 \%(53-85) & 81 \%(66-92) \\ \text { Superonasal RNFL } & 0.82(0.73-0.89) & 42 \%(07-59) & 67 \%(46-84) \\ \text { Upper nasal RNFL } & 0.83(0.73-0.89) & 39 \%(02-65) & 70 \%(51-88) \\ \text { Lower nasal RNFL } & 0.78(0.68-0.87) & 34 \%(05-53) & 58 \%(33-81) \\ \text { Inferonasal RNFL } & 0.87(0.78-0.92) & 61 \%(43-77) & 73 \%(53-85) \\ \text { Inferotemporal RNFL } & 0.90(0.82-0.95) & 67 \%(51-88) & 84 \%(69-93) \\ \text { Lower temporal RNFL } & 0.66(0.56-0.78) & 19 \%(08-36) & 44 \%(25-64) \\ \text { Average GCC thickness } & 0.91(0.83-0.95) & 55 \%(38-83) & 86 \%(70-95) \\ \text { Superior GCC thickness } & 0.84(0.75-0.91) & 41 \%(25-64) & 77 \%(56-90) \\ \text { Inferior GCC thickness } & 0.91(0.84-0.96) & 63 \%(46-78) & 80 \%(61-91)\end{array}$

AUC: area under the receiver operating characteristic curve; RNFL: retinal nerve fiber layer; GCC: ganglion cell complex.

Figure 2 shows the ROC curves of the structural and vessel density measurements of ONH, peripapillary and macular regions. Figure 3 shows the sensitivity at $95 \%$ specificity of the vessel density and structural measurements at different severities of glaucomatous VF loss. Sensitivities of structural measurements of the $\mathrm{ONH}$ and macular regions were better than the vessel densities of the corresponding regions over the whole range of glaucoma severity. Sensitivity of the average peripapillary vessel density was lesser than that of the RNFL thickness in early glaucoma.

(a)

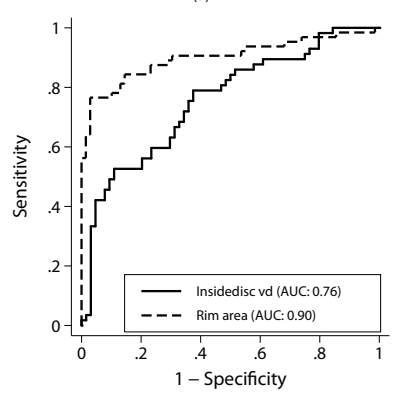

(b)

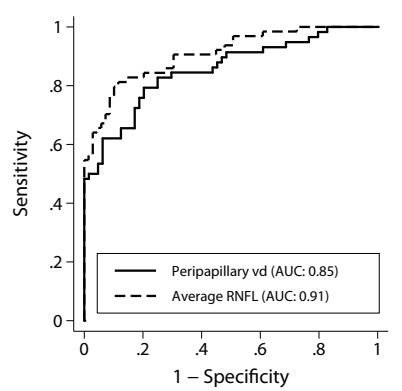

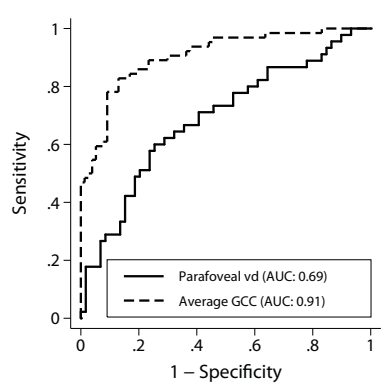

Figure 2. Receiver operating characteristic curves of (a) optic nerve head vessel density (solid line) and rim area (dotted line), (b) average peripapillary vessel density (solid line) and retinal nerve fiber 
layer thickness (dotted line) and (c) average parafoveal vessel density (solid line) and ganglion cell complex thickness (dotted line). vd: vessel density; RNFL: retinal nerve fiber layer; GCC: ganglion cell complex.
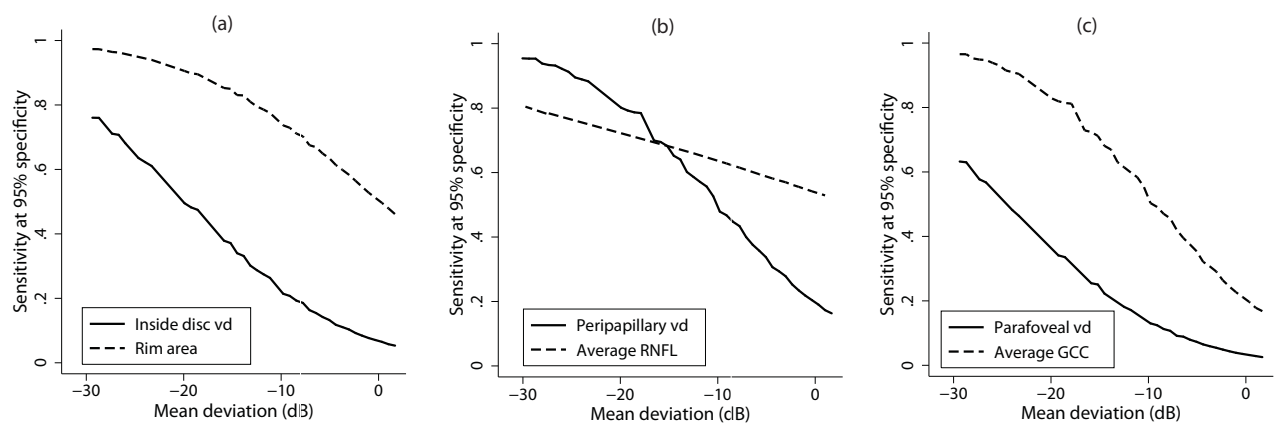

Figure 3. Sensitivity at $95 \%$ specificity of (a) optic nerve head vessel density (solid line) and rim area (dotted line), (b) average peripapillary vessel density (solid line) and retinal nerve fiber layer thickness (dotted line) and (c) average parafoveal vessel density (solid line) and ganglion cell complex thickness (dotted line) according to mean deviation on visual fields. vd: vessel density; RNFL: retinal nerve fiber layer; GCC: ganglion cell complex.

The entire analysis was redone considering one eye of subjects who contributed both eyes for the earlier analysis and similar results were found. When considering the better eye of the PACG patients for analysis (median MD: $-8.1 \mathrm{~dB}$ ), the AUC of inside disc density (0.77) was significantly lesser $(p=0.005)$ than $\mathrm{ONH}$ rim area (0.91), peripapillary vessel density $(0.86)$ was similar $(p=0.51)$ to peripapillary RNFL thickness (0.89), and parafoveal vessel density (0.64) was significantly lesser $(p=0.002)$ than the average GCC thickness (0.88). When considering the worse eye of the glaucoma patients for analysis (median MD: $-10.4 \mathrm{~dB}$ ), the AUC of inside disc vessel density (0.77) was significantly lesser $(p=0.005)$ than $\mathrm{ONH}$ rim area (0.91), peripapillary vessel density $(0.85)$ was similar $(p=0.29)$ to peripapillary RNFL thickness (0.90), and parafoveal vessel density $(0.70)$ was significantly lesser $(p<0.001)$ than the average GCC thickness (0.95).

The entire analysis also was done considering VF changes as the definition of glaucoma. The analysis included 77 control eyes and 56 glaucoma eyes (14 preperimetric eyes in PACG group were excluded from the glaucoma group here and 5 eyes in the PAC group that had an "outside normal limit" GHT result and 
a probability of PSD $\leq 5 \%$ were included in the glaucoma group). AUC of inside disc vessel density $(0.78)$ was significantly lesser $(p=0.02)$ than ONH rim area (0.89), peripapillary vessel density $(0.88)$ was similar $(p=0.97)$ to peripapillary RNFL thickness (0.88), and parafoveal vessel density (0.74) was significantly lesser $(p=0.001)$ than the average GCC thickness (0.91).

\section{DISCUSSION}

The present study compares vessel density measurements in PACD with control eyes using OCTA. Vessel densities in the ONH, peripapillary and macular regions on PACG eyes were significantly lower than control eyes. We also compared the structural and vascular measurements in PAC eyes with a history of high IOP but a healthy disc on clinical exam, with control eyes. This group of PAC eyes with high IOP provided an opportunity to evaluate if the raised IOP first affected the structural or the vascular measurements. We found that the vessel densities in all regions of PAC eyes were statistically similar to that of the control eyes while the peripapillary RNFL in the superotemporal sector was statistically significantly thinner in the PAC eyes. These results may suggest that high IOP affects the RNFL measurements earlier than vessel densities. Contrary to this, the observation of a statistically significantly thinner RNFL in a single sector of PAC eyes could however be by chance. Longitudinal studies in PAC eyes are required to validate this finding.

Comparing the diagnostic abilities in PACG, the AUC and sensitivity at high specificities of ONH rim area and macular GCC thickness were significantly better than the corresponding vessel densities within each of these regions. Diagnostic ability of peripapillary RNFL thickness, though greater than the peripapillary vessel density, was not statistically significant. Unlike POAG, there is limited literature on the utility of vessel densities of OCTA in PACG. In a recent study, we compared the diagnostic ability of peripapillary vessel densities of OCTA with the peripapillary RNFL thickness measurements in PACG eyes and found that the diagnostic abilities were comparable. ${ }^{21}$ There are, however, no previous studies 
evaluating the vessel densities of the $\mathrm{ONH}$ and the macular regions in PACG eyes.

An interesting finding of the present study was the variability in the diagnostic ability of the peripapillary RNFL and vessel density across the different stages of PACG as shown in Figure 3b. In the early stages of VF loss, the sensitivity of peripapillary RNFL thickness was better than that of vessel density for fixed specificities. This reiterates our hypothesis that the vessel density reduction occurs later than the structural changes in PACG and is likely to be secondary to the loss of retinal ganglion cells (RGC) and their axons. However, in later stages of glaucoma (MD on VF between -20 to $-30 \mathrm{~dB}$ ), the diagnostic ability of peripapillary vessel density appears to be better than that of the peripapillary RNFL thickness. The limited usefulness of traditional OCT in advanced glaucoma has been attributed to the floor effect, with the RNFL and GCC thicknesses showing little change with increasing severity of glaucoma. ${ }^{22}$ The vessel density measurements may be devoid of the floor effect and may be better at detecting progression in advanced glaucoma. Future work should evaluate the usefulness of OCTA in advanced glaucoma.

Glaucoma in the current study was defined solely on the neuroretinal rim and RNFL changes on clinical examination and stereo photographs of the optic discs. This may have biased the diagnostic ability of structural measurements compared to vessel density measurements. Hence, a separate analysis was performed considering VF changes as the definition of glaucoma; the results were the same. Therefore, these results are likely to represent true superiority of structural measurements over vessel density measurements for diagnosing glaucoma.

Another possible confounder in the results can be the effect of anti-glaucoma medications on the vessel density measurements. Though there are no studies in literature evaluating this using OCTA, a previous meta-analysis has reported increased ocular blood flow with topical carbonic anhydrase inhibitors. ${ }^{23}$ It is therefore possible that the anti-glaucoma medications increase the vessel density measurements of OCTA independent of the IOP lowering effect. This can reduce the difference in the vessel density measurements between control and treated 
PACD eyes and thereby the diagnostic abilities. Future studies should evaluate the effect of different classes of anti-glaucoma medications on the vessel density measurements of OCTA and account for this while comparing vessel density and structural measurements in glaucoma.

There are some limitations of the OCTA technology and the study design which need to be considered while interpreting the results. A significant number of OCTA scans had to be excluded because of poor quality. Some of the previous studies have also reported high number of poor quality images with OCTA ${ }^{6,24-26}$ and real time tracking is being used currently to reduce artifacts with OCTA imaging. ${ }^{27}$ The OCTA algorithm, in its current form, includes large vessels along with capillaries in its estimation of vessel density. The software also does not provide further insights into the nature of vascular changes such as attenuation, drop-out, etc. The technology also does not evaluate the choroidal vasculature. These details would provide a better understanding of the vascular changes in glaucoma. Another possible limitation of the current study was that we did not measure the blood pressure of the subjects or record their anti-hypertensive medication. However, we recorded the history of hypertension and found that the number of subjects with hypertension was similar in the PACD and the control groups. A previous study also has shown no relationship between blood pressure readings and peripapillary vessel densities on OCTA. ${ }^{6}$ The peripapillary vessel densities can also be affected by parapapillary atrophy (PPA). ${ }^{28}$ We did not record the presence of PPA or its extent in our subjects.

In conclusion, the diagnostic abilities of OCTA vessel density measurements of the $\mathrm{ONH}$ and the macular regions in PACG were significantly lower than the ONH rim area and the macular GCC thickness measurements, respectively, while that of peripapillary vessel densities were comparable to the peripapillary RNFL thickness measurements. Vessel density measurements were comparable, while the superotemporal RNFL thickness was significantly lower in PAC compared to control eyes. This suggests that the structural changes in PACD occur earlier than the vessel density reduction seen with OCTA and future studies should longitudinally evaluate this. Future longitudinal studies should also evaluate the utility of OCTA in detecting progression. 


\section{REFERENCES}

1. Jia Y, Tan O, Tokayer J, et al. Split-spectrum amplitude-decorrelation angiography with optical coherence tomography. Opt Express 2012;20:4710-4725.

2. Jia Y, Morrison JC, Tokayer J, et al. Quantitative OCT angiography of optic nerve head blood flow. Biomed Opt Express 2012;3:3127-3137.

3. Jia $Y$, Wei $E$, Wang $X$, et al. Optical coherence tomography angiography of optic disc perfusion in glaucoma. Ophthalmology 2014;121:1322-1332.

4. Wang $\mathrm{X}$, Jiang $\mathrm{C}$, Ko $\mathrm{T}$, et al. Correlation between optic disc perfusion and glaucomatous severity in patients with open-angle glaucoma: an optical coherence tomography angiography study. Graefes Arch Clin Exp Ophthalmol 2015;253:1557-1564.

5. Leveque PM, Zeboulon P, Brasnu E, Baudouin C, Labbe A. Optic Disc Vascularization in Glaucoma: Value of Spectral-Domain Optical Coherence Tomography Angiography. J Ophthalmol 2016;20166956717.

6. Liu L, Jia Y, Takusagawa HL, et al. Optical Coherence Tomography Angiography of the Peripapillary Retina in Glaucoma. JAMA Ophthalmol 2015;133:1045-1052.

7. Yarmohammadi A, Zangwill LM, Diniz-Filho A, et al. Optical Coherence Tomography Angiography Vessel Density in Healthy, Glaucoma Suspect, and Glaucoma Eyes. Invest Ophthalmol Vis Sci 2016;57:OCT451-459.

8. Rao HL, Pradhan ZS, Weinreb RN, et al. Regional Comparisons of Optical Coherence Tomography Angiography Vessel Density in Primary Open-Angle Glaucoma. Am J Ophthalmol 2016;171:75-83.

9. Cheng JW, Zong Y, Zeng YY, Wei RL. The prevalence of primary angle closure glaucoma in adult Asians: a systematic review and meta-analysis. PLoS One 2014;9:e103222.

10. Weinreb RN, Friedman D.S., eds. Angle closure and angle closure glaucoma, Reports and Consensus Statements of the 3rd Global AIGS Consensus Meeting on Angle Closure Glaucoma The Hague, The Netherlands: Kugler Publications, 2006:1-20.

11. Kraus MF, Potsaid B, Mayer MA, et al. Motion correction in optical coherence tomography volumes on a per A-scan basis using orthogonal scan patterns. Biomed Opt Express 2012;3:1182-1199.

12. Garway-Heath DF, Poinoosawmy D, Fitzke FW, Hitchings RA. Mapping the visual field to the optic disc in normal tension glaucoma eyes. Ophthalmology 2000;107:1809-1815.

13. Rao HL, Zangwill LM, Weinreb RN, Sample PA, Alencar LM, Medeiros FA. Comparison of different spectral domain optical coherence tomography scanning areas for glaucoma diagnosis. Ophthalmology 2010;117:1692-1699.

14. Rao HL, Leite MT, Weinreb RN, et al. Effect of disease severity and optic disc size on diagnostic accuracy of RTVue spectral domain optical coherence tomograph in glaucoma. Invest Ophthalmol Vis Sci 2011;52:1290-1296.

15. Glynn RJ, Rosner B. Accounting for the correlation between fellow eyes in regression analysis. Arch Ophthalmol 1992;110:381-387.

16. Zhou XH, Obuchowski NA, McClish DK. Analysis of correlated ROC data. In: Zhou XH, 
Obuchowski NA, McClish DK, eds. Statistical Methods in Diagnostic Medicine. New York:John Wiley \& Sons, Inc.;2002:274-306.

17. Alonzo TA, Pepe MS. Distribution-free ROC analysis using binary regression techniques. Biostatistics 2002;3:421-432.

18. Pepe MS. Three approaches to regression analysis of receiver operating characteristic curves for continuous test results. Biometrics 1998;54:124-135.

19. Pepe MS. An interpretation for the ROC curve and inference using GLM procedures. Biometrics 2000;56:352-359.

20. Pepe MS. The Statistical Evaluation of Medical Tests for Classification and Prediction. Oxford, UK: Oxford University Press; 2003:130-67.

21. Rao HL, Kadambi SV, Weinreb RN, et al. Diagnostic ability of peripapillary vessel density measurements of optical coherence tomography angiography in primary open-angle and angle-closure glaucoma. Br J Ophthalmol. doi: 10.1136/bjophthalmol-2016-309377.

22. Hood DC, Kardon RH. A framework for comparing structural and functional measures of glaucomatous damage. Prog Retin Eye Res 2007;26:688-710.

23. Siesky B, Harris A, Brizendine E, et al. Literature review and meta-analysis of topical carbonic anhydrase inhibitors and ocular blood flow. Surv Ophthalmol 2009;54:33-46.

24. Suh MH, Zangwill LM, Manalastas PI, et al. Optical Coherence Tomography Angiography Vessel Density in Glaucomatous Eyes with Focal Lamina Cribrosa Defects. Ophthalmology 2016;123:2309-2317.

25. Hollo G. Intrasession and Between-Visit Variability of Sector Peripapillary Angioflow Vessel Density Values Measured with the Angiovue Optical Coherence Tomograph in Different Retinal Layers in Ocular Hypertension and Glaucoma. PLoS One 2016;11:e0161631.

26. Spaide RF, Fujimoto JG, Waheed NK. Image Artifacts in Optical Coherence Tomography Angiography. Retina 2015;35:2163-2180.

27. Camino A, Zhang M, Gao SS, et al. Evaluation of artifact reduction in optical coherence tomography angiography with real-time tracking and motion correction technology. Biomed Opt Express 2016;7:3905-3915.

28. Akagi T, lida Y, Nakanishi H, et al. Microvascular Density in Glaucomatous Eyes With Hemifield Visual Field Defects: An Optical Coherence Tomography Angiography Study. Am J Ophthalmol 2016;168:237-249. 


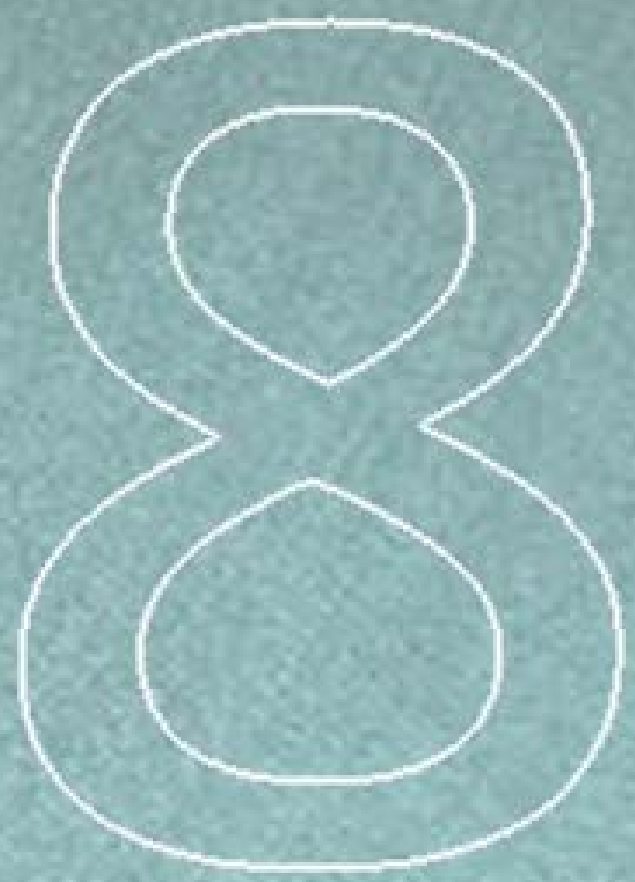

\section{Structure-function relationship with peripapillary vessel density in glaucoma}

Relationship of optic nerve structure and function to peripapillary vessel density measurements of optical coherence tomography angiography in glaucoma

Harsha L Rao, Zia S Pradhan, Robert N Weinreb, Srilakshmi Dasari, Mohammed Riyazuddin, Sharanya Raveendran, Narendra K Puttaiah, Jayasree P Venugopal, Dhanaraj A S Rao, Sathi Devi, Kaweh Mansouri, Carroll AB Webers.

J Glaucoma 2017 Jun;26(6):548-554 


\section{ABSTRACT}

Purpose: To evaluate the sectoral and global structure-structure (vessel densityretinal nerve fiber layer [RNFL] thickness) and structure-function (vessel densityvisual sensitivity loss) relationships of peripapillary vessel density measurements on optical coherence tomography angiography (OCTA) in primary open angle glaucoma (POAG) and to determine if fractional polynomial (FP) models characterize the relationships better than linear models.

Methods: In a cross-sectional study, structure-structure and structure-function relationships of peripapillary vessel densities were determined in 227 eyes of 143 subjects (63 control and 164 POAG eyes) who had undergone standard automated perimetry and OCT testing within 6 months of each other, using linear and FP models. FP model evaluates the relationship between the dependent and the best-fitting fractional powers of the independent variable. Strength of relationship was reported as coefficient of determination $\left(R^{2}\right)$.

Results: $\mathrm{R}^{2}$ values for structure-structure associations using linear models ( 0.53 for superotemporal sector, 0.61 for inferotemporal and 0.53 for average measurements) were significantly less $(p<0.05)$ than that determined using FP models $\left(0.57,0.65\right.$ and 0.55 respectively). $R^{2}$ values for structure-function associations using linear models $(0.35$ for superotemporal vessel densityinferotemporal visual sensitivity loss, 0.49 for inferotemporal vessel densitysuperotemporal visual sensitivity loss and 0.39 for average vessel density-average visual sensitivity loss) were significantly less than that determined using FP models $(0.43,0.58$ and 0.47 respectively).

Conclusions: The inferotemporal peripapillary vessel density showed the strongest association with the corresponding RNFL thickness and visual sensitivity loss in the global and sectoral regions studied. The FP models were significantly better than linear models in describing these relationships. 


\section{INTRODUCTION}

Primary open angle glaucoma (POAG) is a chronic progressive optic neuropathy resulting from the apoptosis of retinal ganglion cells (RGC). ${ }^{1}$ Although increased intraocular pressure (IOP) is the predominant risk factor for RGC death, ${ }^{2}$ reduced ocular perfusion has also been proposed to play a role in the pathogenesis of glaucoma. ${ }^{3,4}$ Earlier studies have measured ocular perfusion using a variety of techniques and have shown a reduction in optic nerve head (ONH) perfusion in patients with glaucoma. However, each of these techniques has certain limitations. ${ }^{5}$

Optical coherence tomography (OCT) angiography non-invasively images the blood vessels of the ONH and retina in-vivo. ${ }^{6}$ Vessel density measurements provided by OCT angiography (OCTA) have been reported to be repeatable and reproducible. ${ }^{7-11}$ A reduction in vessel densities within the $\mathrm{ONH}$, the peripapillary region and the macula has been demonstrated in patients with glaucoma using OCTA. ${ }^{7-10,12,13,14}$

Although the diagnostic ability of OCTA in glaucoma has been determined previously, studies evaluating the relationship of vessel densities of OCTA with the functional (visual sensitivity) measurements on perimetry (structure-function relationship) are limited. ${ }^{10,15,16}$ Two such studies have evaluated the relationship between the global vessel density measurement (average peripapillary vessel density) and the global functional measurements (mean deviation and mean sensitivity) without evaluating the sectors separately. ${ }^{10,16}$ Since the vessel density around the disc varies in a similar manner to the retinal nerve fiber layer (RNFL) thickness, and because glaucoma preferentially affects the poles of the $\mathrm{ONH}, \mathrm{a}$ sectoral analysis of vessel density to corresponding visual fields is likely to provide better information. One study has evaluated the relationship between vascular structure and visual function in the superotemporal and inferotemporal sectors by using linear models. ${ }^{15}$ However, the nature of relationships between vessel densities and RNFL or visual sensitivity measurements is not yet well established and linear models may not characterize these relationships adequately. ${ }^{16}$ 
The purpose of this study was to evaluate the sectoral and global structurestructure (vessel density-RNFL thickness) and structure-function relationships of peripapillary vessel density measurements on OCTA in POAG and to determine whether fractional polynomial models or linear models better characterize these relationships.

\section{METHODS}

This was a prospective, cross-sectional study conducted at Narayana Nethralaya, a tertiary eye care center in Bengaluru, South India between July 2015 and July 2016. The methodology adhered to the tenets of the Declaration of Helsinki for research involving human subjects. Written informed consent was obtained from all participants and the study was approved by the Institute's Ethics Committee.

Participants of the study included control subjects and POAG patients. Control subjects were either patients who consulted for a routine eye examination or refractive error, or were recruited from the hospital staff. Control subjects had no family history of glaucoma, IOP $\leq 21 \mathrm{~mm} \mathrm{Hg}$ on Goldmann applanation tonometry, normal anterior and posterior segment on clinical examination by an ophthalmologist and non-glaucomatous optic discs, as assessed by glaucoma experts on masked examination of stereoscopic optic disc photographs. POAG patients had open anterior chamber angles on gonioscopy and glaucomatous changes on $\mathrm{ONH}$ examination (neuroretinal rim narrowing, notching and retinal nerve fiber layer defects) as documented by glaucoma experts on dilated examination and confirmed by experts on stereoscopic optic disc photographs. Neither pre-treatment IOP, nor visual field changes were used to define POAG. Inclusion criteria for all participants were age $\geq 18$ years, corrected distance visual acuity of $20 / 40$ or better and refractive error within $\pm 5 D$ sphere and $\pm 3 D$ cylinder. Exclusion criteria were presence of any media opacities that prevented good quality OCT scans, or any retinal or neurological disease other than glaucoma, which could confound the evaluation. Eyes with a history of trauma 
or inflammation were also excluded. All participants underwent a comprehensive ocular examination, which included a detailed medical history, corrected distance visual acuity measurement, slit-lamp biomicroscopy, Goldmann applanation tonometry, gonioscopy, dilated fundus examination, stereo-disc photography, visual field (VF) examination, OCT and OCTA imaging with RTVue-XR SD-OCT (Optovue Inc., Fremont, CA).

Stereoscopic optic disc photographs were obtained by trained technicians using a digital fundus camera (Kowa nonmyd WX, Kowa Company, Ltd., Japan). Each optic disc photograph was evaluated independently by two glaucoma experts (HLR and NKP) in a masked manner to determine the presence of glaucomatous changes (focal or diffuse neuroretinal rim thinning, localized notching or RNFL defects). The experts were masked to all the clinical data, visual field data and the fellow eye data. Discrepancy in the classification between the two experts was adjudicated by a third glaucoma expert (ZSP).

VF examination was performed using a Humphrey Field analyzer II, model $720 \mathrm{i}$ (Zeiss Humphrey Systems, Dublin, CA), with the Swedish interactive threshold algorithm (SITA) standard 24-2 program. VFs were considered reliable if the fixation losses were less than $20 \%$, and the false positive and false negative response rates were less than $15 \%$. VFs were also excluded if the glaucoma hemifield test reported "generalized reduction of sensitivity". VF findings were not used for defining glaucoma or controls. Structure-function relationship was evaluated using the Garway-Heath map, which divides the VF into 6 sectors (nasal, inferonasal, inferotemporal, superotemporal, superonasal and temporal sectors). ${ }^{17}$ Structurefunction relationship was evaluated using the visual sensitivity loss in different sectors. Visual sensitivity loss in sectors was calculated by first converting the $\mathrm{dB}$ scale values at each test location on the total deviation numerical plot to a linear scale (reciprocal of Lambert scale) using the following formula.

$$
\frac{1}{\text { Lambert }}=(10)^{0.1 \times d B}
$$

Then values from all test points within a particular sector were averaged. The 
average visual sensitivity loss per sector was used as such in the linear scale and also converted back to the $\mathrm{dB}$ scale for the analysis.

All subjects underwent the peripapillary RNFL thickness measurements on RTVueXR SD-OCT using the ONH scan. The ONH scan protocol has been explained in detail previously. ${ }^{18,19}$ The peripapillary RNFL is divided into 8 sectors (uppertemporal, superotemporal, superonasal, upper-nasal, lower-nasal, inferonasal, inferotemporal and lower-temporal), each of 45 degrees and the thickness in each of the sector and the overall average is provided.

OCTA imaging of the optic disc region was performed using RTVue-XR SD-OCT (AngioVue, v2015.100.0.33). The procedure of OCTA imaging with RTVue-XR has been detailed previously. ${ }^{14}$ In brief, it uses an $840 \mathrm{~nm}$ diode laser source, with an A-scan rate of $70 \mathrm{kHz}$ per second. Imaging is performed using a set of 2 scans; one vertical priority and one horizontal priority raster volumetric scan. The optic disc scan covered an area of $4.5 \times 4.5 \mathrm{~mm}$ and was centered on the optic disc. An orthogonal registration algorithm is used to produce merged 3-dimensional OCT angiograms. ${ }^{20}$ Vessel density is defined as the percentage area occupied by the large vessels and microvasculature in a particular region. Vessel densities are calculated over the entire scan area, i.e., whole enface disc, as well as the peripapillary regions. The peripapillary region is defined as a $0.75 \mathrm{~mm}$-wide elliptical annulus extending from the optic disc boundary. The peripapillary vessel density is analyzed from the "Radial Peripapillary Capillary (RPC) segment" which extends from the ILM to the posterior boundary of the nerve fiber layer. In addition to estimation of the average vessel density for the entire peripapillary region, it is also divided into 6 sectors based on the Garway-Heath map and the vessel densities in each sector is calculated. ${ }^{17}$

Image quality was assessed for all OCT and OCTA scans. Poor quality images, which were defined as those with a signal strength index (SSI) less than 35 or images with motion artifacts and segmentation errors were excluded from the analysis. All the examinations for a particular subject were performed within 6 months of each other. 
In this study, we evaluated the structure-structure and the structurefunction relationships of the vessel densities in the peripapillary region and its inferotemporal and superotemporal sectors, with the corresponding RNFL thickness and visual sensitivity.

\section{STATISTICAL ANALYSIS}

Descriptive statistics included mean and standard deviation for normally distributed variables and median and inter-quartile range (IQR) for non-normally distributed variables. Shapiro-Wilk test was used to test for the normality distribution of continuous variables.

Structure-structure and structure-function associations were investigated by using linear $(y=a x+b)$ and fractional polynomial (FP) regression (up to second degree or two dimensions) between vessel density, RNFL thickness and visual sensitivity loss measurements. Unlike the linear regression which evaluates the relationship between the dependent and the independent variable in their original form, FP regression evaluates the relationship between the dependent and the best-fitting fractional powers of the independent variable. ${ }^{21}$ Conventional polynomial functions such as linear and the quadratic are often used to evaluate the relationship between dependent and independent variables. However, these functions are limited in their range of curve shapes. Cubic and higher-order curves often produce undesirable artifacts such as edge effects and waves. Fractional polynomials increase the flexibility afforded by the family of conventional polynomial models by allowing logarithms, non-integer powers and powers to be repeated. ${ }^{21}$ As non-positive values are not analysed in FP regression, the visual sensitivity loss values in decibel scale (which contained negative numbers) were converted to positive numbers by adding $34 \mathrm{~dB}$ uniformly before introduction into the FP models. Forty-four different FP models (which also included the linear and the quadratic models) were fit for each relationship and the best fit FP model was chosen depending on the deviance value. The partial $F$ test was used to compare the goodness of fit of the linear regression and the best FP regression model. As 
measurements from both eyes of the same subject are likely to be correlated, clustered sandwich estimator technique was used in the regression models and the cluster of data for the study subject was considered as the primary sampling unit when estimating the coefficients and standard errors. ${ }^{22,23}$ Statistical analyses were performed using commercial software (Stata ver. 13.1; StataCorp, College Station, TX). A $p$ value of $\leq 0.05$ was considered statically significant.

\section{RESULTS}

Two hundred and twenty-eight eyes of 143 subjects ( 64 eyes of 44 control subjects and 164 eyes of 99 POAG patients) were enrolled into the study. Of these, 26 unreliable VFs, 16 poor quality OCTA scans and 12 poor quality RNFL scans (one eye had unreliable VF and poor quality OCTA and RNFL scan) were excluded from the analysis. Table 1 shows the demographic, clinical, vessel density, RNFL thickness and visual sensitivity parameters of the two groups. POAG patients were significantly older than control subjects. SSI of the OCTA and OCT scans were significantly greater in control as compared to the POAG patients. All the vessel density, RNFL and visual field measurements were significantly worse in the POAG cohort as compared to the control group.

Table 1. Clinical features, visual field parameters, vessel density and structural measurements of the participants. All values represent median and interquartile range unless specified.

\begin{tabular}{lccc}
\hline & $\begin{array}{c}\text { Control group } \\
\text { (63 eyes, } \\
\mathbf{4 4} \text { subjects) }\end{array}$ & $\begin{array}{c}\text { POAG group } \\
\text { (164 eyes, } \\
\mathbf{9 9} \text { patients) }\end{array}$ & P \\
\hline Age (years) & 54.5 & 65.6 & $<0.001$ \\
Gender (male:female) & $(45.6,62.8)$ & $(58.5,70.8)$ & 0.26 \\
Sphere (D) & $26: 18$ & $68: 31$ & 0.19 \\
& 0.5 & 0.0 & \\
Cylinder (D) & $(0,1.5)$ & $(-0.75,1.25)$ & 0.04 \\
& -0.5 & -0.75 & \\
Pseudophakia (n, \%) & $(-0.75,0)$ & $(-1,-0.25)$ & $<0.001$ \\
IOP at the scanning visit (mm Hg)* & 2 & 38 & 0.13
\end{tabular}




\section{Chapter 8}

\begin{tabular}{|c|c|c|c|}
\hline Hypertension (yes:no) & $11: 33$ & $34: 65$ & 0.27 \\
\hline Diabetes mellitus (yes:no) & $11: 33$ & $32: 67$ & 0.38 \\
\hline \multicolumn{4}{|c|}{ OCTA parameters } \\
\hline SSI (Optic disc scan)* & $59.7 \pm 9.3$ & $51.3 \pm 7.9$ & $<0.001$ \\
\hline Whole enface vessel density (disc scan, \%) & $\begin{array}{c}55.1 \\
(53.8,57.1)\end{array}$ & $\begin{array}{c}45.9 \\
(42.1,49.8)\end{array}$ & $<0.001$ \\
\hline Average Peripapillary vessel density (\%) & $\begin{array}{c}61.2 \\
(59.4,62.7)\end{array}$ & $\begin{array}{c}53.9 \\
(48.8,58.6)\end{array}$ & $<0.001$ \\
\hline Inferotemporal vessel density (\%) & $\begin{array}{c}65.8 \\
(63.0,68.1)\end{array}$ & $\begin{array}{c}52.7 \\
(42.1,59.9)\end{array}$ & $<0.001$ \\
\hline Superotemporal vessel density (\%) & $\begin{array}{c}64.8 \\
(61.2,67.9)\end{array}$ & $\begin{array}{c}58.6 \\
(50.2,63.5)\end{array}$ & $<0.001$ \\
\hline \multicolumn{4}{|c|}{ OCT parameters } \\
\hline SSI (ONH scan) & $\begin{array}{c}59 \\
(54,68)\end{array}$ & $\begin{array}{c}50 \\
(44,57)\end{array}$ & $<0.001$ \\
\hline Average Peripapillary RNFL thickness $(\mu \mathrm{m})$ & $\begin{array}{c}102 \\
(97,105)\end{array}$ & $\begin{array}{c}79 \\
(70,85)\end{array}$ & $<0.001$ \\
\hline Inferotemporal RNFL thickness $(\mu \mathrm{m})$ & $\begin{array}{c}134 \\
(128,141)\end{array}$ & $\begin{array}{c}84 \\
(71,104)\end{array}$ & $<0.001$ \\
\hline Superotemporal RNFL thickness ( $\mu \mathrm{m})$ & $\begin{array}{c}137 \\
(128,143) \\
\end{array}$ & $\begin{array}{c}104 \\
(87,119) \\
\end{array}$ & $<0.001$ \\
\hline \multicolumn{4}{|c|}{ Visual field parameters } \\
\hline Mean deviation (dB) & $\begin{array}{c}-1.0 \\
(-2.4,0.0)\end{array}$ & $\begin{array}{c}-6.1 \\
(-12.2,-3.3)\end{array}$ & $<0.001$ \\
\hline Pattern standard deviation (dB) & $\begin{array}{c}1.8 \\
(1.5,2.2)\end{array}$ & $\begin{array}{c}5.4 \\
(3.2,9.8)\end{array}$ & $<0.001$ \\
\hline Visual field index (\%) & $\begin{array}{c}99 \\
(98,99)\end{array}$ & $\begin{array}{c}87 \\
(69,94)\end{array}$ & $<0.001$ \\
\hline Inferotemporal visual sensitivity loss (dB scale) & $\begin{array}{c}-1.0 \\
(-1.7,0.0)\end{array}$ & $\begin{array}{c}-3.2 \\
(-5.0,-1.9)\end{array}$ & $<0.001$ \\
\hline Inferotemporal visual sensitivity loss (linear scale) & $\begin{array}{c}0.8 \\
(0.7,1.0)\end{array}$ & $\begin{array}{c}0.5 \\
(0.3,0.6)\end{array}$ & $<0.001$ \\
\hline Superotemporal visual sensitivity loss (dB scale) & $\begin{array}{c}-1.0 \\
(-2.1,-0.1)\end{array}$ & $\begin{array}{c}-5.7 \\
(-11.7,-3.2)\end{array}$ & $<0.001$ \\
\hline Superotemporal visual sensitivity loss (linear scale) & $\begin{array}{c}0.8 \\
(0.6,1.0) \\
\end{array}$ & $\begin{array}{c}0.3 \\
(0.1,0.5) \\
\end{array}$ & $<0.001$ \\
\hline
\end{tabular}

POAG: primary open angle glaucoma; D: diopter; dB: decibel; IOP: intraocular pressure; OCT: optical coherence tomography; OCTA: OCT angiography; SSI: signal strength index; ONH: optic nerve head; RNFL: retinal nerve fiber layer; *mean \pm standard deviation.

The results of the univariate linear and FP regression evaluating the relationship between vessel density and RNFL thickness measurements are summarized in Table 2. The best FP regression model for the superotemporal structure-structure 
relationship was the one with the term (superotemporal RNFL) ${ }^{-1}$ substituted for superotemporal RNFL and this model was statistically significantly better $(p<0.001)$ than the linear regression model. Similarly, the best FP regression model for the inferotemporal structure-structure relationship contained the term (inferotemporal RNFL) ${ }^{-0.5}$ and this model was statistically significantly better $(p<0.001)$ than the linear regression model. The best FP regression model for the relationship between peripapillary average vessel density and RNFL thickness contained the term (average RNFL) ${ }^{-0.5}$ and this model was statistically significantly better $(p=0.05)$ than the linear regression model. Figure 1 shows these relationships graphically. The discordance between the linear and the FP fits appeared to be greater at the ends of the measurement spectrum of vessel density and RNFL thickness.

Table 2. Structure-structure association of peripapillary vessel densities with corresponding sector retinal nerve fiber layer thickness measurements.

\begin{tabular}{lcccc}
\hline \multirow{2}{*}{ Sector } & \multicolumn{2}{c}{ Linear model } & \multicolumn{2}{c}{ Fractional polynomial model } \\
\cline { 2 - 5 } & $\mathbf{R}^{\mathbf{2}}(\mathbf{p}$ value $)$ & AIC & $\mathbf{R}^{\mathbf{2}}$ ( value $)$ & AIC \\
\hline Superotemporal & $0.53(<0.001)$ & 1277.7 & $0.57(<0.001)$ & 1260.5 \\
Inferotemporal & $0.61(<0.001)$ & 1367.3 & $0.65(<0.001)$ & 1349.4 \\
Average & $0.53(<0.001)$ & 1137.9 & $0.55(<0.001)$ & 1131.8 \\
\hline
\end{tabular}

$\mathrm{R}^{2}$ : coefficient of determination; AIC: Akaike's information criterion.
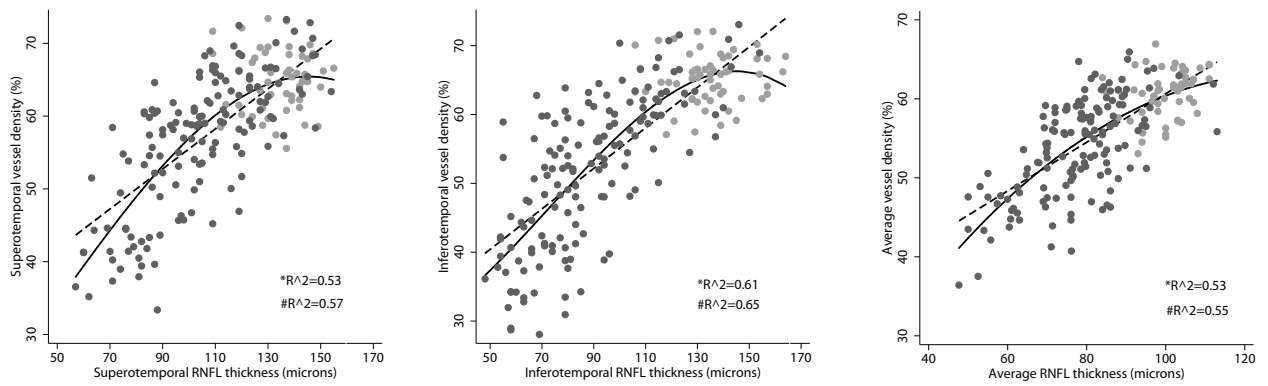

Figure 1. Structure-structure relationship between the vessel density and retinal nerve fiber layer (RNFL) thickness measurements. Dashed line represents the linear fit and the solid line represents the fractional polynomial fit. Grey markers represent control subjects and black represent glaucoma patients. 
The results of the univariate linear and FP regression evaluating the relationship between vessel density and visual sensitivity loss (in decibel and linear scales) are summarized in Table 3. The FP regression models were significantly better $(p<0.001)$ than the linear regression models for all the pairwise relationships. The relationship between inferotemporal sector vessel density and superotemporal sector visual sensitivity loss showed the best $\mathrm{R}^{2}$ values. The best FP regression model for the relationship between inferotemporal vessel density and superotemporal visual sensitivity loss in decibel scale contained the term (superotemporal sensitivity loss) ${ }^{3}$ and the best model for the same relationship in linear scale contained the term (superotemporal sensitivity loss) ${ }^{(1,2)}$. Figure 2 shows these relationships graphically.

Table 3. Structure-function associations of peripapillary vessel densities with visual sensitivity loss of the corresponding sector.

\begin{tabular}{|c|c|c|c|c|c|c|c|c|}
\hline \multirow{3}{*}{$\begin{array}{l}\text { Vessel density } \\
\text { Sector }\end{array}$} & \multicolumn{4}{|c|}{ Loss of visual sensitivity in decibel scale } & \multicolumn{4}{|c|}{ Loss of visual sensitivity in linear scale } \\
\hline & \multicolumn{2}{|c|}{ Linear model } & \multicolumn{2}{|c|}{ FP model } & \multicolumn{2}{|c|}{ Linear model } & \multicolumn{2}{|c|}{ FP model } \\
\hline & $\begin{array}{l}R^{2}(p \\
\text { value })\end{array}$ & $\mathrm{AIC}$ & $\begin{array}{l}R^{2}(p \\
\text { value })\end{array}$ & AIC & $\begin{array}{l}R^{2}(p \\
\text { value })\end{array}$ & AIC & $\begin{array}{l}R^{2}(p \\
\text { value })\end{array}$ & AIC \\
\hline Superotemporal & $\begin{array}{c}0.35 \\
(<0.001)\end{array}$ & 1275.1 & $\begin{array}{c}0.43 \\
(<0.001)\end{array}$ & 1256.6 & $\begin{array}{c}0.30 \\
(<0.001)\end{array}$ & 1273.7 & $\begin{array}{c}0.42 \\
(<0.001)\end{array}$ & 1241.7 \\
\hline Inferotemporal & $\begin{array}{c}0.49 \\
(<0.001)\end{array}$ & 1327.6 & $\begin{array}{c}0.58 \\
(<0.001)\end{array}$ & 1294.0 & $\begin{array}{c}0.50 \\
(<0.001)\end{array}$ & 1311.8 & $\begin{array}{c}0.59 \\
(<0.001)\end{array}$ & 1274.3 \\
\hline Average & $\begin{array}{c}0.39 \\
(<0.001)\end{array}$ & 1130.9 & $\begin{array}{c}0.47 \\
(<0.001)\end{array}$ & 1122.0 & $\begin{array}{c}0.40 \\
(<0.001)\end{array}$ & 1131.0 & $\begin{array}{c}0.47 \\
(<0.001)\end{array}$ & 1107.8 \\
\hline
\end{tabular}

FP: Fractional polynomial; $\mathrm{R}^{2}$ : coefficient of determination; AIC: Akaike's information criterion.
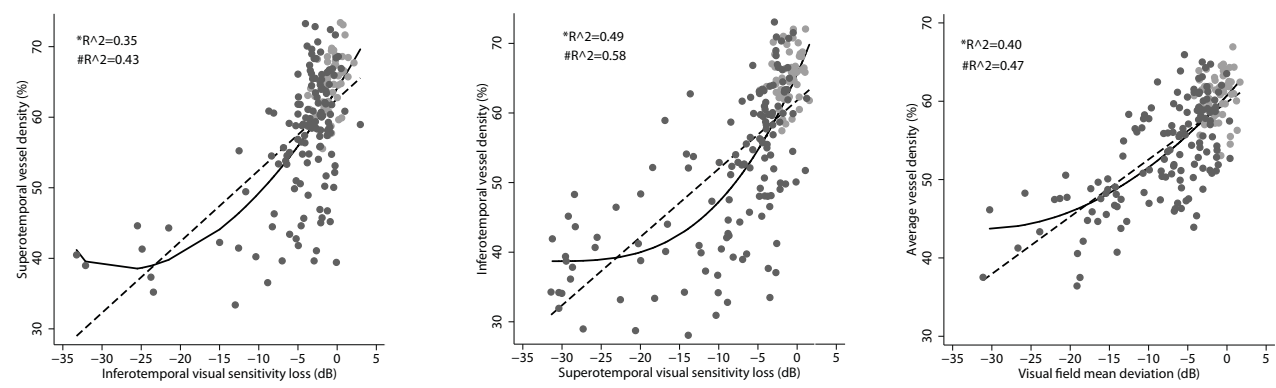

Figure 2.Structure-function relationship between the vessel density measurements and visual sensitivity loss on decibel scale. Dashed line represents the linear fit and the solid line represents 
the fractional polynomial fit. Grey markers represent control subjects and black represent glaucoma patients.

The results of the univariate linear and FP regression evaluating the relationship between RNFL thickness and visual sensitivity loss (in decibel and linear scales) are summarized in Table 4. The FP regression models were significantly better $(p<0.001)$ than the linear regression models for all the pairwise relationships. The relationship between inferotemporal sector RNFL thickness and superotemporal sector visual sensitivity loss showed the best $\mathrm{R}^{2}$ values. The best FP regression model for the relationship between inferotemporal RNFL thickness and superotemporal visual sensitivity loss in decibel scale contained the term (superotemporal sensitivity loss) ${ }^{(3,3)}$ and the best model for the same relationship in linear scale contained the term (superotemporal sensitivity loss) ${ }^{(1,3)}$. Figure 3 shows these relationships graphically.

Table 4. Structure-function associations of peripapillary retinal nerve fiber layer (RNFL) thickness with visual sensitivity loss of the corresponding sector.

\begin{tabular}{|c|c|c|c|c|c|c|c|c|}
\hline \multirow{3}{*}{ RNFL Sector } & \multicolumn{4}{|c|}{ Loss of visual sensitivity in decibel scale } & \multicolumn{4}{|c|}{ Loss of visual sensitivity in linear scale } \\
\hline & \multicolumn{2}{|c|}{ Linear model } & \multicolumn{2}{|c|}{ FP model } & \multicolumn{2}{|c|}{ Linear model } & \multicolumn{2}{|c|}{ FP model } \\
\hline & $\begin{array}{c}R^{2} \\
\text { ( } p \text { value) }\end{array}$ & AIC & $\begin{array}{c}\mathrm{R}^{2} \\
\text { ( } p \text { value) }\end{array}$ & AIC & $\begin{array}{c}\mathrm{R}^{2} \\
\text { ( } \mathrm{p} \text { value) }\end{array}$ & $\mathrm{AIC}$ & $\begin{array}{c}\mathrm{R}^{2} \\
\text { ( } p \text { value) }\end{array}$ & AIC \\
\hline Superotemporal & $\begin{array}{c}0.37 \\
(<0.001)\end{array}$ & 1602.6 & $\begin{array}{c}0.46 \\
(<0.001)\end{array}$ & 1574.3 & $\begin{array}{c}0.34 \\
(<0.001)\end{array}$ & 1585.5 & $\begin{array}{c}0.46 \\
(<0.001)\end{array}$ & 1550.8 \\
\hline Inferotemporal & $\begin{array}{c}0.46 \\
(<0.001)\end{array}$ & 1698.8 & $\begin{array}{c}0.63 \\
(<0.001)\end{array}$ & 1632.7 & $\begin{array}{c}0.58 \\
(<0.001)\end{array}$ & 1630.6 & $\begin{array}{c}0.65 \\
(<0.001)\end{array}$ & 1600.9 \\
\hline Average & $\begin{array}{c}0.42 \\
(<0.001)\end{array}$ & 1385.7 & $\begin{array}{c}0.56 \\
(<0.001)\end{array}$ & 1359.1 & $\begin{array}{c}0.47 \\
(<0.001)\end{array}$ & 1362.2 & $\begin{array}{c}0.53 \\
(<0.001)\end{array}$ & 1343.8 \\
\hline
\end{tabular}

FP: Fractional polynomial; $\mathrm{R}^{2}$ : coefficient of determination; $\mathrm{Cl}$ : confidence interval; AIC: Akaike's information criterion. 

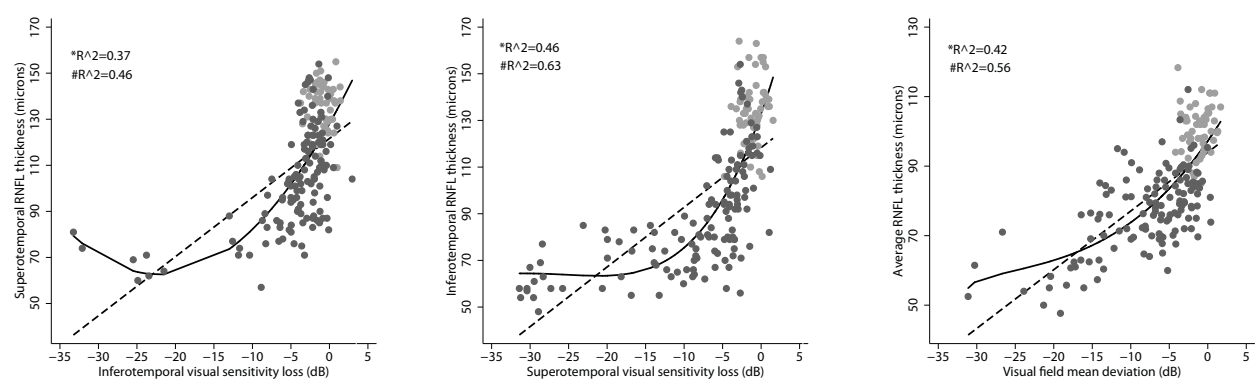

Figure 3. Structure-function relationship between the retinal nerve fiber layer (RNFL) thickness measurements and visual sensitivity loss on decibel scale. Dashed line represents the linear fit and the solid line represents the fractional polynomial fit. Grey markers represent control subjects and black represent glaucoma patients.

We performed a power analysis and found that our study had a power of $>85 \%$ at an alpha value of 0.05 to detect the differences in the $R^{2}$ values that were found between the FP and linear models for all the relationships.

\section{DISCUSSION}

There is limited literature on the relationship between peripapillary vessel density as measured by OCTA and RNFL thickness or visual sensitivity. ${ }^{10,15,16}$ These previous studies evaluated the relationship between the global vessel density measurement and the global functional measurements ${ }^{10,16}$ or have used linear models to fit the relationships. ${ }^{10,15}$ Liu et al, in 12 glaucoma eyes, reported a correlation coefficient $(r)$ of $0.35\left(R^{2}=0.12 ; p=0.26\right)$ between average peripapillary vessel density and RNFL thickness, and a $r$ of $0.68\left(R^{2}=0.46 ; p=0.02\right)$ between average peripapillary vessel density and visual field MD. ${ }^{10}$ Akagi et al, in a study including 21 normal eyes, 34 POAG eyes without high myopia and 26 POAG eyes with high myopia reported a $r$ of $0.53\left(R^{2}=0.28 ; p<0.001\right)$ between average peripapillary vessel density and average RNFL thickness. ${ }^{15}$ In the 60 glaucomatous eyes, they reported a $r$ of $0.47\left(R^{2}=0.22 ; p=0.004\right)$ between average peripapillary vessel density and MD and a $r$ of $0.38\left(R^{2}=0.14 ; p=0.003\right)$ 
between average RNFL thickness and MD. ${ }^{15}$ Yarmohammadi et al reported a $\mathrm{R}^{2}$ of $0.53(p<0.001)$ between average peripapillary vessel density and average RNFL thickness, $0.54(p<0.001)$ between average peripapillary vessel density and MD and 0.36 between average RNFL thickness and MD. ${ }^{16}$ Our results are closer to the results reported in the study by Yarmohammadi et al. The differences in the strength of associations between average peripapillary vessel densities and RNFL thickness or MD seen in these studies can be explained by the differences in the disease characteristics of the study population and the OCT systems used.

Akagi et al, performed a sectoral analysis of the associations of peripapillary vessel densities and reported a $r$ of $0.37\left(R^{2}=0.14 ; p=0.001\right)$ between superotemporal vessel density and superotemporal RNFL thickness and a r of $0.75\left(R^{2}=0.56 ; p<0.001\right)$ between inferotemporal vessel density and inferotemporal RNFL thickness. ${ }^{15}$ They also reported a r of $0.49\left(R^{2}=0.24 ; p<0.001\right)$ between superotemporal vessel density and inferior total deviation values, $0.77\left(R^{2}=0.59 ; p<0.001\right)$ between superotemporal RNFL thickness and inferior total deviation value, $0.59\left(R^{2}=0.35\right.$; $p<0.001)$ between inferotemporal vessel density and superior total deviation value and $0.80\left(R^{2}=0.64 ; p<0.001\right)$ between inferotemporal RNFL thickness and superior total deviation value. ${ }^{15}$ The structure-function relationships were stronger with the inferotemporal compared to the superotemporal sector vessel density and RNFL thickness measurements. ${ }^{15}$ This is similar to the results found in our study.

The goodness-of-fit of the structure-function relationships with the traditional OCT measurements (RNFL thickness and ONH rim area) have been extensively studied using various methodologies. Garway-Heath et $\mathrm{al}^{24}$ reported that the visual sensitivity expressed in linear scale defined the structure-function relationship better than visual sensitivities expressed in a decibel (dB) scale. Bowd et $\mathrm{al}^{25}$ showed that a linear fit between structure and function with visual sensitivity expressed in $d B$ scale was comparable to a logarithmic fit in describing the structure-function relationship. Hood and Kardon ${ }^{26}$ showed that a simple linear model considering function in terms of visual sensitivity loss (total deviation plot on standard automated perimetry) can adequately describe the structure-function relationship. In contrast, the nature of the structure-function 
relationships with vessel density measurements of OCTA have not been well characterised. Yarmohammadi et al therefore compared the structure-function fits of linear and quadratic models $\left(y=a x^{2}+b x+c\right)$ and found that the quadratic models better represent the relationships. The association between average peripapillary vessel density and MD had a $\mathrm{R}^{2}$ of 0.54 on linear modelling and 0.62 on quadratic modelling. The same between average RNFL thickness and MD was 0.36 and 0.44 respectively. We used FP models instead of quadratic models because FP models provide greater range of curve shapes compared to quadratic fits. We found that the FP regression models provided significantly stronger structure-structure and structure-function associations compared to the linear models. FP models provided a stronger association between structure and function even when the visual sensitivity loss was expressed in linear scale. The linear model doesn't provide the strongest association between structure and function even when both are expressed in the same scale because the relationship is unlikely to be linear over the whole range of measurements (whole range of disease severities). This suggests that there is no benefit of using the linear scale over the dB scale when FP model is used. The polynomial terms were not similar for relationships between either structure-structure or structure-function in different sectors. Structure-structure relationship in the superotemporal sector had (superotemporal RNFL) ${ }^{-1}$ as the polynomial term while that in the inferotemporal sector had (inferotemporal RNFL) ${ }^{-0.5}$. Similar findings were noted with structure-function relationships in different sectors. This suggests that the fits to the structure-structure and structure-function relationships have different shapes in different sectors. Evaluating the associations between vessel density and RNFL thickness, we found that the discordance between the linear and the FP fits was greater at the ends of the measurement spectrum. The FP fit may represent reduced variability of vessel density compared to RNFL thickness measurements in control subjects or a relatively slower reduction in vessel densities in early stages of glaucoma. FP models evaluating the structure-function relationship between vessel density and visual sensitivity loss also demonstrated that the vessel density decrease slows down beyond visual sensitivity loss of $-15 \mathrm{~dB}$. This is similar to the structure-function relationship with RNFL thickness where the RNFL thickness reaches a base level at visual sensitivity loss of -10 to $-15 \mathrm{~dB}$, 
beyond which little change in RNFL thickness is seen. ${ }^{26,27}$ However, the scatter of data points appears to be greater in the relationship between vessel density and visual sensitivity loss (Figure 2) compared to that between RNFL thickness and visual sensitivity loss (Figure 3). One possible reason for this can be a variability in vessel density reduction seen in different subgroups of patients with POAG. A recent study by Suh et al demonstrated that in eyes with similar visual sensitivity loss, the decrease in vessel density that was seen in POAG eyes with focal lamina cribrosa defects was significantly greater than that in POAG eyes without focal lamina cribrosa defects. ${ }^{28}$ The decrease in RNFL thickness, however, was found to be comparable between POAG eyes with and without lamina cribrosa defects. ${ }^{28}$ Understanding the relationship between structure and function helps us to better quantify the severity of glaucoma. Understanding the relationships is also important in detecting progression early. Our results demonstrated that the FP models were significantly better than linear models in fitting all the structurestructure and structure-function relationships. FP models, therefore, appear to be better than other models in bringing out the true relationships between structure and function. Being cross-sectional in design, our study is however, unable to provide information on the nature of longitudinal changes in vasculature or RNFL thickness in normal and glaucoma eyes. Future studies, therefore, should aim to evaluate the associations of the vascular and neuronal structure over time in different phenotypes of POAG.

There are some limitations of the OCTA technology and the study design which need to be considered while interpreting the results. The vessel density measurements evaluated in this study were provided automatically by the software. The OCTA algorithm, in its current form, includes large vessels along with capillaries in its estimation of vessel density. Estimating the capillary density separately may provide greater information. The superotemporal and inferotemporal vessel density sectors in our study were not exactly matched with the corresponding RNFL sectors. This may have affected the results. However, using the GarwayHeath map provided good correspondence between the vessel density and the visual field sectors. Also, OCTA measured vessel density is a surrogate for blood flow but not a true measure of blood flow. Lastly, SSI of the scans is reported to have a significant association with the vessel densities in normal eyes, with the 
densities being significantly greater in scans with higher SSI. ${ }^{29}$ However, there is no consensus on what value constitutes an adequate SSI. Different studies have used different SSI values, ranging from 35 to 50, as cut-offs for good quality scans. ${ }^{10}, 12-14,30$ We analyzed our data considering a SSI value of 50 as the cutoff for good quality scans (136 OCTA and 130 RNFL scans of 55 normal and 99 glaucoma eyes) and found that the results were similar to those with SSI values above 35 . The $\mathrm{R}^{2}$ values of all relationships with both linear and FP models were slightly stronger when scans with a SSI of $\geq 50$ were analysed. The $R^{2}$ value of the FP model for the structure-structure relationship in the inferotemporal sector was 0.67 . The same for the structure-function relationship in the inferotemporal vessel density sector was 0.67 with the visual sensitivity loss expressed in $\mathrm{dB}$ scale and 0.68 with the visual sensitivity loss expressed in linear scale. The $\mathrm{R}^{2}$ value of the FP model for the structure-function relationship in the inferotemporal RNFL sector was 0.67 with the visual sensitivity loss expressed in $\mathrm{dB}$ scale and 0.69 with the visual sensitivity loss expressed in linear scale.

In conclusion, the inferotemporal peripapillary vessel density showed the strongest association with the corresponding RNFL thickness and visual sensitivity loss in the global and sectoral regions studied. The FP models were significantly better than the linear models in describing these relationships.

\section{REFERENCES}

1. Weinreb RN, Khaw PT. Primary open-angle glaucoma. Lancet 2004;363:1711-20.

2. Weinreb RN, Aung T, Medeiros FA. The pathophysiology and treatment of glaucoma: a review. JAMA 2014;311:1901-11.

3. Bonomi L, Marchini G, Marraffa M, et al. Vascular risk factors for primary open angle glaucoma: the Egna-Neumarkt Study. Ophthalmology 2000;107:1287-93.

4. Leske MC, Heijl A, Hyman L, et al. Predictors of long-term progression in the Early Manifest Glaucoma Trial. Ophthalmology 2007;114:1965-72.

5. Weinreb RN, Harris A., ed. Ocular Blood Flow in Glaucoma, The 6th Consensus Report of the World Glaucoma Association. Amsterdam: Kugler Publications, 2009; 19-56.

6. Jia Y, Tan O, Tokayer J, et al. Split-spectrum amplitude-decorrelation angiography with optical coherence tomography. Opt Express 2012;20:4710-25. 
7. Jia Y, Morrison JC, Tokayer J, et al. Quantitative OCT angiography of optic nerve head blood flow. Biomed Opt Express 2012;3:3127-37.

8. Jia $Y$, Wei E, Wang $X$, et al. Optical coherence tomography angiography of optic disc perfusion in glaucoma. Ophthalmology 2014;121:1322-32.

9. Wang X, Jiang C, Ko T, et al. Correlation between optic disc perfusion and glaucomatous severity in patients with open-angle glaucoma: an optical coherence tomography angiography study. Graefes Arch Clin Exp Ophthalmol 2015;253:1557-64.

10. Liu L, Jia Y, Takusagawa HL, et al. Optical Coherence Tomography Angiography of the Peripapillary Retina in Glaucoma. JAMA Ophthalmol 2015;133:1045-52.

11. Yu J, Jiang C, Wang $X$, et al. Macular perfusion in healthy Chinese: an optical coherence tomography angiogram study. Invest Ophthalmol Vis Sci 2015;56:3212-7.

12. Leveque PM, Zeboulon P, Brasnu E, et al. Optic Disc Vascularization in Glaucoma: Value of Spectral-Domain Optical Coherence Tomography Angiography. J Ophthalmol 2016;2016:6956717.

13. Yarmohammadi A, Zangwill LM, Diniz-Filho A, et al. Optical Coherence Tomography Angiography Vessel Density in Healthy, Glaucoma Suspect, and Glaucoma Eyes. Invest Ophthalmol Vis Sci 2016;57:OCT451-9.

14. Rao HL, Pradhan ZS, Weinreb RN, et al. Regional Comparisons of Optical Coherence Tomography Angiography Vessel Density in Primary Open-Angle Glaucoma. Am J Ophthalmol 2016;171:75-83.

15. Akagi T, lida Y, Nakanishi H, et al. Microvascular Density in Glaucomatous Eyes With Hemifield Visual Field Defects: An Optical Coherence Tomography Angiography Study. Am J Ophthalmol 2016;168:237-49.

16. Yarmohammadi A, Zangwill LM, Diniz-Filho A, et al. Relationship between Optical Coherence Tomography Angiography Vessel Density and Severity of Visual Field Loss in Glaucoma. Ophthalmology 2016;123:2498-508.

17. Garway-Heath DF, Poinoosawmy D, Fitzke FW, Hitchings RA. Mapping the visual field to the optic disc in normal tension glaucoma eyes. Ophthalmology 2000;107:1809-15.

18. Rao HL, Zangwill LM, Weinreb RN, et al. Comparison of different spectral domain optical coherence tomography scanning areas for glaucoma diagnosis. Ophthalmology 2010;117:1692-9.

19. Rao HL, Leite MT, Weinreb RN, et al. Effect of disease severity and optic disc size on diagnostic accuracy of RTVue spectral domain optical coherence tomograph in glaucoma. Invest Ophthalmol Vis Sci 2011;52:1290-6.

20. Kraus MF, Potsaid B, Mayer MA, et al. Motion correction in optical coherence tomography volumes on a per A-scan basis using orthogonal scan patterns. Biomed Opt Express 2012;3:1182-99.

21. Royston P, Sauerbrei W. Building multivariable regression models with continuous covariates in clinical epidemiology--with an emphasis on fractional polynomials. Methods Inf Med 2005;44:561-71.

22. Glynn RJ, Rosner B. Accounting for the correlation between fellow eyes in regression analysis. Arch Ophthalmol 1992;110:381-7. 


\section{Chapter 8}

23. Williams RL. A note on robust variance estimation for cluster-correlated data. Biometrics 2000;56:645-6.

24. Garway-Heath DF, Holder GE, Fitzke FW, Hitchings RA. Relationship between electrophysiological, psychophysical, and anatomical measurements in glaucoma. Invest Ophthalmol Vis Sci 2002;43:2213-20.

25. Bowd C, Zangwill LM, Medeiros FA, et al. Structure-function relationships using confocal scanning laser ophthalmoscopy, optical coherence tomography, and scanning laser polarimetry. Invest Ophthalmol Vis Sci 2006;47:2889-95.

26. Hood DC, Kardon RH. A framework for comparing structural and functional measures of glaucomatous damage. Prog Retin Eye Res 2007;26:688-710.

27. Rao HL, Zangwill LM, Weinreb RN, et al. Structure-function relationship in glaucoma using spectral-domain optical coherence tomography. Arch Ophthalmol 2011;129:864-71.

28. Suh MH, Zangwill LM, Manalastas PI, et al. Optical Coherence Tomography Angiography Vessel Density in Glaucomatous Eyes with Focal Lamina Cribrosa Defects. Ophthalmology 2016;123:2309-17.

29. Rao HL, Pradhan ZS, Weinreb RN, et al. Determinants of peripapillary and macular vessel densities measured by optical coherence tomography angiography in normal eyes. J Glaucoma 2017;26:491-497.

30. Rao HL, Kadambi SV, Weinreb RN, et al. Diagnostic ability of peripapillary vessel density measurements of optical coherence tomography angiography in primary open-angle and angle-closure glaucoma. Br J Ophthalmol 2017;101:1066-1070. 


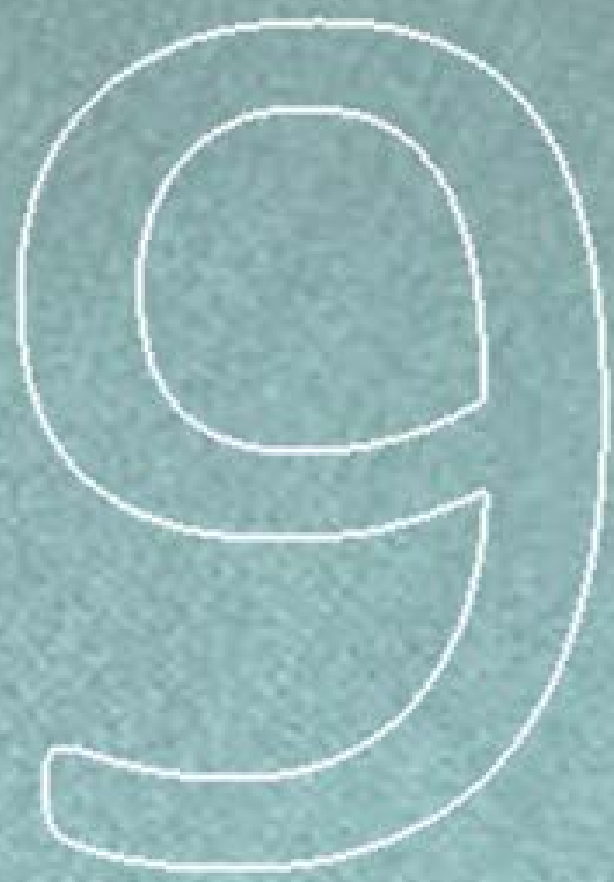

\section{OCT angiography in eyes with disc hemorrhage}

Optical coherence tomography angiography vessel density measurements in eyes with primary open angle glaucoma and disc hemorrhage

Harsha L Rao, Zia S Pradhan, Robert N Weinreb, Srilakshmi Dasari, Mohammed Riyazuddin, Jayasree P Venugopal, Narendra K Puttaiah, Dhanaraj A S Rao, Sathi Devi, Kaweh Mansouri, Carroll AB Webers.

J Glaucoma 2017 Oct;26(10):888-895 


\section{ABSTRACT}

Purpose: To compare the vessel density measurements of optic nerve head $(\mathrm{ONH})$, peripapillary and macular regions in severity-matched primary open angle glaucoma (POAG) eyes with and without disc hemorrhage (DH) using optical coherence tomography (OCT) angiography (OCTA), and to compare their diagnostic abilities with that of the rim area, retinal nerve fiber layer and the ganglion cell complex thickness measurements on OCT.

Methods: In a cross-sectional study, 66 eyes of 46 control subjects, 34 eyes of 33 POAG patients with DH (median mean deviation, MD: $-3.7 \mathrm{~dB}$ ) and 63 eyes of 43 POAG patients without DH (median MD: $-3.8 \mathrm{~dB}$ ) underwent imaging with spectral domain OCT. Area under receiver operating characteristic curves (AUC) and sensitivities at $90 \%$ specificity of vessel density and structural measurements in POAG eyes with DH were compared with those in POAG eyes without DH.

Results: Most of the vessel density and structural measurements were similar $(p>0.05)$ in POAG eyes with and without DH. Whole enface vessel density of the disc scan and inferotemporal peripapillary vessel density showed the best AUC and sensitivity at $90 \%$ specificity both in POAG eyes with $\mathrm{DH}(0.82,56 \%$ and 0.75 , $59 \%)$ and without DH $(0.91,73 \%$ and $0.83,67 \%)$. AUCs and sensitivities of vessel density and structural measurements of POAG eyes with and without DH were statistically similar $(p>0.05)$.

Conclusions: OCTA-measured vessel densities and their diagnostic abilities in POAG eyes with and without DH were similar. This suggests that the cause of DH in POAG is unlikely to be vascular abnormality. 


\section{INTRODUCTION}

Primary open angle glaucoma (POAG) is a chronic progressive optic neuropathy resulting from the apoptosis of the retinal ganglion cells (RGC). ${ }^{1}$ Although increased intraocular pressure (IOP) is the predominant risk factor for RGC death, ${ }^{2}$ reduced ONH perfusion has also been proposed to play a role in the pathogenesis of glaucoma in at least some individuals. ${ }^{3,4}$ Optic disc hemorrhage (DH), which is strongly associated with the development and progression of glaucoma in major clinical trials, ${ }^{5-8}$ is said to be one of the clinical indicator of altered ocular perfusion as it has been associated with systemic vascular diseases, platelet dysfunction, primary vascular dysregulation, vasospasm, and dysfunctional autoregulation of the blood flow to the optic nerve head. ${ }^{9,} 10$ Though the exact pathogenesis of DH is not fully known, it is proposed to be vascular due to the above mentioned associations. In contrast, a few studies have proposed mechanical vascular disruption at the level of the lamina cribrosa, or the margin of optic disc or retinal nerve fiber layer (RNFL) defect as the cause of $\mathrm{DH} .{ }^{11-14}$

Optical coherence tomography (OCT) angiography is a technique of non-invasively imaging of the blood vessels of the optic nerve head (ONH) and retina in-vivo. ${ }^{15}$ Studies have used OCT angiography (OCTA) to demonstrate a reduction of vessel density within the $\mathrm{ONH}$, the peripapillary retina and the macula in patients with POAG ${ }^{16-22}$ However, there are no studies to date on the OCTA vessel density measurements in eyes with $\mathrm{DH}$. We hypothesized that at similar severity of glaucomatous damage, the vessel density reduction on OCTA in POAG would be greater in eyes with $\mathrm{DH}$ compared to eyes without $\mathrm{DH}$, if the cause of $\mathrm{DH}$ is vascular in origin. The purpose of the current study was to compare the vessel density measurements of the $\mathrm{ONH}$, peripapillary and macular regions in POAG eyes with and without DH using OCTA. In addition, the diagnostic abilities of the vessel density and structural measurements of the $\mathrm{ONH}$, peripapillary and macular regions on OCT were also compared in POAG eyes with and without $\mathrm{DH}$. 


\section{METHODS}

This was a prospective, cross-sectional study conducted at Narayana Nethralaya, a tertiary eye care center in Bengaluru, South India between September 2015 and September 2016. The methodology adhered to the tenets of the Declaration of Helsinki for research involving human subjects. Written informed consent was obtained from all participants and the study was approved by the Institute's Ethics Committee.

Participants of the study included control subjects and POAG patients. Control subjects were either hospital staff or subjects who consulted for a routine eye examination or a refractive error. Control subjects had no family history of glaucoma, IOP $\leq 21 \mathrm{~mm} \mathrm{Hg}$, normal anterior and posterior segments on clinical examination by an ophthalmologist and non-glaucomatous optic discs, as assessed by glaucoma experts on masked examination of stereoscopic optic disc photographs. POAG patients had open angles on gonioscopy and glaucomatous changes on optic nerve head examination (neuroretinal rim narrowing, notching and retinal nerve fiber layer defects) as documented by glaucoma experts on dilated examination and confirmed by experts on stereoscopic optic disc photographs. POAG patients were divided into two groups; those showing a $\mathrm{DH}$ in either eye and those showing no DH. DH was defined as an isolated splinter-like or flame-shaped hemorrhage on the optic disc or peripapillary region extending up to the border of the optic disc. DH was documented on disc photographs independently by two experts. The presence or absence of $\mathrm{DH}$ anytime in the past (in the DH and no DH groups respectively) was ascertained by going through the medical charts of all the patients and also previous disc photos when available. The frequency of clinical examinations of most of the POAG patients, noted from the medical charts, was 4 to 6 monthly and that of optic disc photography was yearly. Neither pre-treatment IOP, nor visual field changes were used to define POAG. Inclusion criteria for all participants were age $\geq 18$ years, corrected distance visual acuity of $20 / 40$ or better and refractive error within $\pm 5 D$ sphere and $\pm 3 D$ cylinder. Exclusion criteria were presence of any media opacities that prevented good quality OCT scans, or any retinal (including posterior vitreous detachment, 
retinal vein occlusion, diabetic retinopathy) or neurological disease other than glaucoma, which could confound the evaluations. Eyes with a history of trauma or inflammation were also excluded. All participants underwent a comprehensive ocular examination, which included a detailed medical history, corrected distance visual acuity measurement, slit-lamp biomicroscopy, Goldmann applanation tonometry, gonioscopy, dilated fundus examination, stereoscopic optic disc photography, visual field (VF) examination and OCT imaging with RTVue-XR SDOCT (Optovue Inc., Fremont, CA). In addition to IOP measured on the day of scanning, the pre-treatment IOP (i.e. the IOP noted on the day of initiating antiglaucoma treatment) was documented for all POAG eyes.

Stereoscopic optic disc photographs were obtained by trained technicians using a digital fundus camera (Kowa nonmyd WX, Kowa Company, Ltd., Japan). Each optic disc photograph was evaluated independently by two glaucoma experts (ZSP and $\mathrm{HLR}$ ) in a masked manner to determine the presence of glaucomatous changes (focal or diffuse neuroretinal rim thinning, localized notching or RNFL defects) and the presence of $\mathrm{DH}$. The experts were masked to all the clinical data, visual field data and the fellow eye data. Discrepancy in the classification between the two experts was adjudicated by a third glaucoma expert (NKP).

VF examination was performed using a Humphrey Field analyzer II, model 720i (Zeiss Humphrey Systems, Dublin, CA), with the Swedish interactive threshold algorithm (SITA) standard 24-2 program. VFs were considered reliable if the fixation losses were less than $20 \%$, and the false positive and false negative response rates were less than $15 \%$. VF findings were not considered for defining glaucoma or controls in the primary analysis, but were used for the grading of glaucoma severity. VF was considered abnormal if the pattern standard deviation was abnormal at $p<5 \%$ and the glaucoma hemifield test was outside normal limits.

OCTA imaging of the optic disc region and macula was performed using RTVue-XR SD-OCT (AngioVue, version 2016.1.0.26). The procedure of OCTA imaging with RTVue-XR has been detailed previously. ${ }^{22}$ In brief, it uses an $840 \mathrm{~nm}$ diode laser source, with an A-scan rate of $70 \mathrm{kHz}$ per second. Imaging is performed using a 
set of 2 scans; one vertical priority and one horizontal priority raster volumetric scan. The optic disc scan covers an area of $4.5 \times 4.5 \mathrm{~mm}$ and the macular scan was performed using volumetric scans covering $3 \times 3 \mathrm{~mm}$. An orthogonal registration algorithm is used to produce merged 3-dimensional OCT angiograms. ${ }^{23}$ The software compares the consecutive B-scans at the same location to detect flow using motion contrast, thereby delineating blood vessels. ${ }^{15}$ Vessel density is defined as the percentage area occupied by the large vessels and microvasculature in a particular region. Vessel densities are calculated over the entire scan area, i.e. whole enface disc and whole enface macula, as well as defined areas within each scan as described below. In addition, the software calculates vessel densities in various layers of the retina and the $\mathrm{ONH}$.

In the optic disc scan, the software automatically fits an ellipse to the optic disc margin and calculates the average vessel density within the $\mathrm{ONH}$ (referred to as the inside disc vessel density, Figure 1a). The ONH vessel density is calculated from the "nerve head segment" of the $\mathrm{ONH}$ angiogram. This segment extends from 2000 microns above the internal limiting membrane (ILM) to 150 microns below the ILM. In the same optic disc scan, the peripapillary region is defined as a $0.75 \mathrm{~mm}$-wide elliptical annulus extending from the optic disc boundary and the average vessel density within this region is calculated (Figure 1b). The peripapillary vessel density was analyzed from the "Radial Peripapillary Capillary (RPC) segment" which extends from the ILM to the posterior boundary of the nerve fiber layer. The peripapillary region is divided into 8 sectors, each of 45 degrees, and peripapillary vessel density in each sector is reported. Macular vessel densities were analyzed over a $1.5 \mathrm{~mm}$-wide parafoveal, circular annulus centered on the macula. Macular vessel densities analyzed in this study were of the superficial vascular plexus present in the inner layers of the retina (extending from the ILM to the inner plexiform layer, Figure 1c). The parafoveal region was divided into 2 sectors of $180^{\circ}$ each (superior and inferior sectors). 
(a)

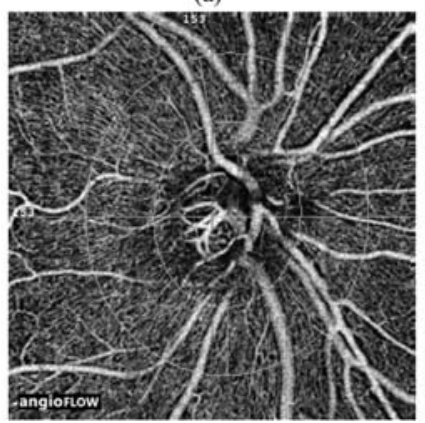

(b)

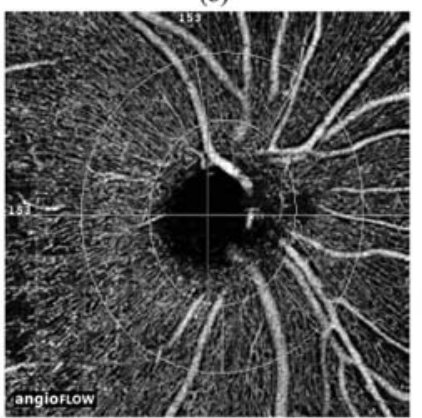

(c)

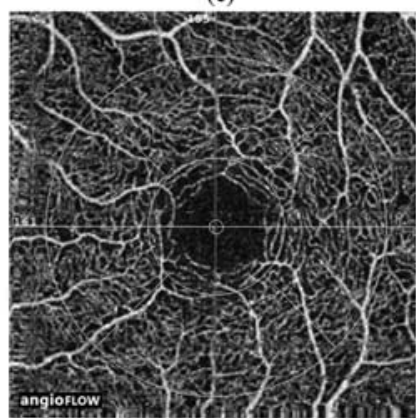

Figure 1. Figure showing the (a) optic nerve head (b) peripapillary and (c) macular optical coherence tomography angiography images and the sectors where vessel densities are calculated. The optic disc vessel density is calculated within the optic nerve head (inner circle on the figure) from the nerve head segment of the en face angiogram (a), peripapillary vessel density over a $0.75 \mathrm{~mm}$-wide elliptical annulus extending from the optic disc boundary from the radial peripapillary capillary segment (b), and superficial macular vessel density over a $1.5 \mathrm{~mm}$-wide circular annulus centered on the macula (c).

All subjects also underwent the traditional ONH, peripapillary RNFL and macular GCC thickness measurements on RTVue-XR SD-OCT using the ONH and the GCC scans. These scan protocols have been explained in detail previously. ${ }^{24,} 25$ OCT parameters analysed in the current study were the neuroretinal rim area, average peripapillary RNFL thickness and the RNFL thickness in 8 sectors corresponding to the 8 peripapillary vessel density sectors, and the average GCC thickness and the GCC thickness in the superior and inferior hemispheres. All the examinations for a particular subject were performed on the same day and all examinations in eyes with DH were performed when the hemorrhage was present. Image quality was assessed for all OCTA and OCT scans. Poor quality images, which were defined as those with a signal strength index (SSI) less than 45 or images with motion artifacts and segmentation errors were excluded from the analysis.

\section{STATISTICAL ANALYSIS}

Descriptive statistics included mean and standard deviation for normally 
distributed variables and median and inter-quartile range (IQR) for nonnormally distributed variables. Shapiro-Wilk test was used to test for the normality distribution of continuous variables. Continuous parameters between the groups were compared using $t$ test if normally distributed and Wilcoxon rank sum test if non-normally distributed. Receiver operating characteristic (ROC) curves were used to describe the ability of vessel density and structural measurements of OCT to discriminate POAG eyes with and without DH from control eyes. Sensitivities at fixed specificities of $90 \%$ were determined for all the parameters. To obtain confidence intervals for area under the ROC curves (AUC) and sensitivities, a bootstrap re-sampling procedure was used $(n=1000$ re-samples). As measurements from both eyes of the same subject are likely to be correlated, the standard statistical methods for parameter estimation can lead to underestimation of standard errors and to confidence intervals that are too narrow. ${ }^{26}$ Therefore, the cluster of data for the study subject was considered as the unit of resampling and bias corrected standard errors were calculated during all estimations. This procedure has been used to adjust for the presence of multiple correlated measurements from the same unit. ${ }^{27,28}$ Z-test was used to compare the AUCs of vessel density and structural parameters in POAG eyes with and without $\mathrm{DH} .{ }^{29,}{ }^{30} \mathrm{ROC}$ regression modeling technique was used to evaluate the sensitivities of OCT measurements at various severities of glaucoma, as based on the mean deviation (MD) of VF. ${ }^{31,32}$

Statistical analyses were performed using commercial software (Stata ver. 13.1; StataCorp, College Station, TX). A p value of $\leq 0.05$ was considered statistically significant.

\section{RESULTS}

One hundred and ninety-eight eyes of 141 subjects ( 50 control subjects, 35 POAG patients with $\mathrm{DH}$ in either eye and 56 POAG patients without $\mathrm{DH}$ ) underwent vascular and structural imaging with OCT. Of these, 7 eyes with unreliable VF and 21 eyes with poor quality of both disc and macular OCTA scans were excluded. 
Additionally, 11 eyes with poor quality disc OCTA scans, 11 eyes with poor quality macular OCTA scans, 14 eyes with poor quality ONH scans, 16 eyes with poor quality disc OCTA and ONH scans, 2 eyes with poor quality ONH and GCC scans, and 6 eyes with poor quality disc OCTA, ONH and GCC scans were also excluded. Sixty-six eyes of 46 control subjects, 34 eyes of 33 POAG patients with DH and 70 eyes of 46 POAG patients without DH remained for the analysis. Of these, 7 POAG eyes without $\mathrm{DH}$, which were either from patients too young or had greater severity of VF loss compared to patients with $\mathrm{DH}$, were excluded to match the two POAG groups for age and glaucoma severity (based on mean deviation of VF). The final analysis included 130 good quality OCTA disc scans, 152 OCTA macula scans, $125 \mathrm{ONH}$ scans and 155 GCC scans. The median follow-up noted from the medical charts of POAG patients with DH was 2.1 years (IQR: 0.3-5.0) and POAG patients without DH was 0.7 years $(0.2-1.8)$. DH was noted in inferior or inferotemporal quadrant of optic disc in 20 eyes. As recorded from the medical charts, DH was noted once during the follow-up in 26 eyes, twice in 5 eyes and thrice in 3 eyes. Eight POAG eyes with DH (23.5\%) and 13 POAG eyes without DH (20.6\%) had a "within normal limit" or a "borderline" glaucoma hemifield test (GHT) result, and / or the probability value of pattern standard deviation (PSD) $>5 \%$ on VF (preperimetric glaucoma). In the DH group, 4 eyes (11.8\%) were not on any anti-glaucoma medications, 14 eyes $(41 \%)$ were on topical beta blockers, 8 (23.5\%) on alpha agonists, 12 (38.2\%) on carbonic anhydrase inhibitors and 19 (58.8\%) on prostaglandin analogues (either as a monotherapy or as combination therapy) at the time of OCT scanning. In the POAG eyes without $\mathrm{DH}$, these figures were 9 (14.3\%), 14 (22.2\%), 14 (22.2\%), $13(20.6 \%)$ and 35 (55.6\%) respectively. Significantly greater number of POAG eyes with DH were on beta blockers $(p=0.05)$ and carbonic anhydrase inhibitors $(p=0.06)$ compared to those without DH. Table 1 shows the clinical, VF, vessel density and structural measurements of the included subjects. There were significantly more females in the $\mathrm{DH}$ group. IOP at the scanning visit was significantly lower in POAG eyes with $\mathrm{DH}$ compared to POAG eyes without $\mathrm{DH}$ group $(p=0.003)$. Pre-treatment IOP was also lower in POAG eyes with DH compared to that without $\mathrm{DH}(\mathrm{p}=0.09)$. Most of the vessel density and structural measurements in the POAG eyes with and without $\mathrm{DH}$ were significantly lower than in the control group. Most of the vessel 
density measurements (except the inferonasal and lower temporal peripapillary vessel densities) were similar in POAG eyes with and without DH. Most of the structural measurements (except the neuroretinal rim area, inferonasal RNFL thickness, average and inferior GCC thickness) were also similar in POAG eyes with and without $\mathrm{DH}$. Control subjects were significantly younger than the POAG patients, and the SSI of the ONH scan of OCT was significantly greater in the control subjects compared to that in POAG patients. AUCs and sensitivities at fixed specificities were therefore calculated after adjusting for these differences using covariate-adjustment as proposed by Pepe. ${ }^{33}$

Table 1. Clinical features, visual field parameters, vessel density and structural measurements of the participants. All values represent mean \pm standard deviation unless specified. $P$ value represents the comparison between primary open angle glaucoma ( $P O A G)$ eyes with and without disc hemorrhage (DH).

\begin{tabular}{|c|c|c|c|c|}
\hline & $\begin{array}{c}\text { Control } \\
\text { subjects } \\
\text { (66 eyes, } \\
46 \text { subjects) }\end{array}$ & $\begin{array}{l}\text { POAG with } \\
\text { DH (34 eyes, } \\
33 \text { subjects) }\end{array}$ & $\begin{array}{c}\text { POAG without } \\
\text { DH } \\
\text { (63 eyes, } \\
43 \text { subjects) }\end{array}$ & $\mathbf{P}$ \\
\hline Age (years)* & $\begin{array}{c}59.7 \\
(53.6,66.1)\end{array}$ & $\begin{array}{c}65.6 \\
(60.3,70.0)\end{array}$ & $\begin{array}{c}66.0 \\
(57.2,71.2)\end{array}$ & 0.93 \\
\hline Gender (male:female) & $25: 21$ & $11: 22$ & $30: 13$ & 0.002 \\
\hline Sphere (D)* & $\begin{array}{c}0.75 \\
(0,1.25)\end{array}$ & $\begin{array}{c}1 \\
(0.25,1.75)\end{array}$ & $\begin{array}{c}0.25 \\
(-0.5,1.25)\end{array}$ & 0.06 \\
\hline Cylinder (D)* & $\begin{array}{c}-0.5 \\
(-1,0)\end{array}$ & $\begin{array}{c}-0.5 \\
(-1,-0.25)\end{array}$ & $\begin{array}{c}-0.75 \\
(-1,-0.25)\end{array}$ & 0.50 \\
\hline Pseudophakia (n, \%) & $\begin{array}{c}9 \\
(13.6 \%)\end{array}$ & $\begin{array}{c}12 \\
(35.3 \%)\end{array}$ & $\begin{array}{c}9 \\
(14.3 \%)\end{array}$ & 0.02 \\
\hline Optic disc area $\left(\mathrm{mm}^{2}\right)^{*}$ & $\begin{array}{c}2.28 \\
(1.98,2.57)\end{array}$ & $\begin{array}{c}2.29 \\
(2.03,2.52)\end{array}$ & $\begin{array}{c}2.23 \\
(2.01,2.61)\end{array}$ & 0.86 \\
\hline IOP at the scanning visit $(\mathrm{mm} \mathrm{Hg})$ & $14.9 \pm 2.6$ & $14.6 \pm 3.9$ & $17.4 \pm 3.9$ & 0.003 \\
\hline Pre-treatment IOP (mm Hg) & & $18.6 \pm 4.6$ & $21.1 \pm 5.8$ & 0.09 \\
\hline Hypertension (yes:no) & $16: 30$ & $19: 14$ & $18: 25$ & 0.17 \\
\hline Diabetes mellitus (yes:no) & $14: 32$ & $15: 18$ & $14: 29$ & 0.25 \\
\hline Mean deviation $(\mathrm{dB})^{*}$ & $\begin{array}{c}-0.8 \\
(-3.0,-0.3)\end{array}$ & $\begin{array}{c}-3.7 \\
(-6.3,-2.5)\end{array}$ & $\begin{array}{c}-3.8 \\
(-7.5,-2.8)\end{array}$ & 0.90 \\
\hline
\end{tabular}




\begin{tabular}{|c|c|c|c|c|}
\hline Pattern standard deviation $(\mathrm{dB})^{*}$ & $\begin{array}{c}1.8 \\
(1.5,2.4)\end{array}$ & $\begin{array}{c}4.8 \\
(2.9,8.9)\end{array}$ & $\begin{array}{c}4.7 \\
(2.6,9.5)\end{array}$ & 0.90 \\
\hline Visual field index (\%)* & $\begin{array}{c}99 \\
(98,99)\end{array}$ & $\begin{array}{c}90 \\
(80,96)\end{array}$ & $\begin{array}{c}90 \\
(72,96)\end{array}$ & 0.86 \\
\hline \multicolumn{5}{|c|}{ OCTA parameters } \\
\hline SSI (Optic disc scan) & $56.0 \pm 7.1$ & $54.7 \pm 6.3$ & $54.2 \pm 5.7$ & 0.73 \\
\hline $\begin{array}{l}\text { Whole enface vd } \\
\text { (disc scan, \%) }\end{array}$ & $54.0 \pm 2.9$ & $48.5 \pm 4.6$ & $46.5 \pm 5.9$ & 0.14 \\
\hline Inside disc vd (\%) & $48.2 \pm 4.8$ & $43.1 \pm 4.8$ & $42.6 \pm 6.6$ & 0.73 \\
\hline Average Peripapillary vd (\%) & $62.1 \pm 3.0$ & $57.6 \pm 5.5$ & $56.2 \pm 6.7$ & 0.39 \\
\hline Upper temporal vd (\%) & $62.9 \pm 4.6$ & $61.9 \pm 5.2$ & $59.5 \pm 7.6$ & 0.15 \\
\hline Superotemporal vd (\%) & $64.9 \pm 4.4$ & $58.1 \pm 9.2$ & $59.6 \pm 8.3$ & 0.47 \\
\hline Superonasal vd (\%) & $59.5 \pm 5.5$ & $56.7 \pm 7.3$ & $54.9 \pm 7.9$ & 0.34 \\
\hline Upper nasal vd (\%) & $58.2 \pm 5.3$ & $55.4 \pm 8.8$ & $54.2 \pm 7.0$ & 0.53 \\
\hline Lower nasal vd (\%) & $57.4 \pm 4.9$ & $54.4 \pm 6.7$ & $54.1 \pm 8.2$ & 0.91 \\
\hline Inferonasal vd (\%) & $62.1 \pm 5.0$ & $59.2 \pm 7.9$ & $54.4 \pm 8.7$ & 0.02 \\
\hline Inferotemporal vd (\%) & $64.7 \pm 4.0$ & $55.3 \pm 11.3$ & $54.7 \pm 10.4$ & 0.81 \\
\hline Lower temporal vd (\%) & $58.6 \pm 4.7$ & $59.4 \pm 7.0$ & $55.7 \pm 7.4$ & 0.04 \\
\hline SSI (Macula scan) & $61.1 \pm 6.7$ & $60.7 \pm 7.2$ & $61.9 \pm 6.0$ & 0.41 \\
\hline $\begin{array}{l}\text { Whole enface vd } \\
\text { (macula scan, \%) }\end{array}$ & $47.5 \pm 3.8$ & $44.6 \pm 4.6$ & $44.8 \pm 3.5$ & 0.87 \\
\hline Parafoveal vd (\%) & $49.5 \pm 3.9$ & $47.1 \pm 5.1$ & $47.4 \pm 3.7$ & 0.81 \\
\hline Superior vd (\%) & $49.2 \pm 4.4$ & $47.1 \pm 5.1$ & $46.6 \pm 4.7$ & 0.68 \\
\hline Inferior vd (\%) & $49.3 \pm 4.7$ & $46.9 \pm 5.4$ & $46.2 \pm 4.2$ & 0.50 \\
\hline \multicolumn{5}{|c|}{ OCT parameters } \\
\hline SSI (ONH scan) & $57.5 \pm 6.4$ & $53.5 \pm 6.4$ & $54.3 \pm 6.3$ & 0.60 \\
\hline Neuroretinal rim area $\left(\mathrm{mm}^{2}\right)$ & $1.35 \pm 0.25$ & $0.89 \pm 0.21$ & $0.70 \pm 0.20$ & $<0.001$ \\
\hline Peripapillary RNFL thickness $(\mu \mathrm{m})$ & $101 \pm 9$ & $82 \pm 10$ & $79 \pm 11$ & 0.29 \\
\hline Upper temporal RNFL $(\mu \mathrm{m})$ & $77 \pm 10$ & $68 \pm 9$ & $68 \pm 10$ & 0.98 \\
\hline Superotemporal RNFL $(\mu \mathrm{m})$ & $137 \pm 15$ & $106 \pm 24$ & $106 \pm 19$ & 0.94 \\
\hline Superonasal RNFL $(\mu \mathrm{m})$ & $113 \pm 22$ & $92 \pm 18$ & $88 \pm 20$ & 0.48 \\
\hline Upper nasal RNFL $(\mu \mathrm{m})$ & $85 \pm 13$ & $71 \pm 14$ & $71 \pm 14$ & 0.94 \\
\hline Lower nasal RNFL $(\mu \mathrm{m})$ & $76 \pm 11$ & $64 \pm 10$ & $64 \pm 11$ & 0.88 \\
\hline Inferonasal RNFL ( $\mu \mathrm{m})$ & $118 \pm 18$ & $95 \pm 13$ & $83 \pm 19$ & 0.006 \\
\hline
\end{tabular}




$\begin{array}{lcccc}\text { Inferotemporal RNFL }(\mu \mathrm{m}) & 135 \pm 16 & 97 \pm 22 & 91 \pm 26 & 0.33 \\ \quad \text { Lower temporal RNFL }(\mu \mathrm{m}) & 68 \pm 10 & 64 \pm 9 & 63 \pm 11 & 0.68 \\ \text { SSI (GCC scan) } & 61.8 \pm 7.8 & 62.9 \pm 8.0 & 61.9 \pm 7.1 & 0.52 \\ \text { Average GCC thickness }(\mu \mathrm{m}) & 96 \pm 8 & 82 \pm 9 & 78 \pm 9 & 0.05 \\ \quad \text { Superior GCC thickness }(\mu \mathrm{m}) & 95 \pm 8 & 84 \pm 10 & 82 \pm 9 & 0.26 \\ \text { Inferior GCC thickness }(\mu \mathrm{m}) & 96 \pm 8 & 81 \pm 12 & 75 \pm 12 & 0.03\end{array}$

$\mathrm{DH}$ : disc hemorrhage; D: diopter; dB: decibel; IOP: intraocular pressure; OCT: optical coherence tomography; OCTA: OCT angiography; SSI: signal strength index; vd: vessel density; ONH: optic nerve head; RNFL: retinal nerve fiber layer; GCC: ganglion cell complex; ${ }^{*}$ median and inter-quartile range.

The AUCs and sensitivities at $90 \%$ specificity of the vessel density measurements to differentiate POAG eyes with and without DH from control eyes are shown in Table 2. Whole enface vessel density of the disc scan and inferotemporal peripapillary vessel density showed the best AUC and sensitivity at $90 \%$ specificity both in POAG eyes with and without DH. AUCs of vessel densities in POAG eyes with $\mathrm{DH}$ were statistically similar $(p>0.05)$ to that in POAG eyes without $\mathrm{DH}$. The AUCs and sensitivities at $90 \%$ specificity of the structural measurements to differentiate POAG eyes with and without DH from control eyes are shown in Table 3. Neuroretinal rim area and average RNFL thickness showed the best AUC and sensitivity at $90 \%$ specificity both in POAG eyes with and without DH. AUCs of structural measurements in POAG eyes with DH were statistically similar $(p>0.05)$ to that in POAG eyes without $\mathrm{DH}$.

Table 2. Diagnostic ability of vessel density parameters of optical coherence tomography angiography in differentiating primary open angle glaucoma (POAG) eyes with and without disc hemorrhage (DH) from control eyes (figures in parenthesis represent $95 \%$ confidence intervals).

\begin{tabular}{lcccc}
\hline \multirow{2}{*}{ Vessel density } & \multicolumn{2}{c}{ POAG with DH } & \multicolumn{2}{c}{ POAG without DH } \\
\cline { 2 - 5 } & AUC & $\begin{array}{c}\text { Sensitivity at 90\% } \\
\text { specificity }\end{array}$ & AUC & $\begin{array}{c}\text { Sensitivity at 90\% } \\
\text { specificity }\end{array}$ \\
\hline Whole enface vd (disc scan) & 0.82 & $56 \%$ & 0.91 & $73 \%$ \\
& $(0.70-0.93)$ & $(27-84)$ & $(0.82-0.96)$ & $(52-88)$ \\
Inside disc vd & 0.77 & $39 \%$ & 0.74 & $43 \%$ \\
& $(0.65-0.87)$ & $12-63)$ & $(0.61-0.85)$ & $(23-60)$ \\
Average Peripapillary vd & 0.74 & $40 \%$ & 0.81 & $56 \%$ \\
& $(0.58-0.86)$ & $(17-72)$ & $(0.72-0.89)$ & $(31-77)$
\end{tabular}




\begin{tabular}{ccccc} 
Superotemporal vd & 0.71 & $44 \%$ & 0.71 & $41 \%$ \\
& $(0.56-0.84)$ & $(19-64)$ & $(0.56-0.81)$ & $(22-60)$ \\
Inferotemporal vd & 0.75 & $59 \%$ & 0.83 & $67 \%$ \\
& $(0.60-0.87)$ & $(38-81)$ & $(0.72-0.91)$ & $(45-83)$ \\
Whole enface vd (macula scan) & 0.67 & $30 \%$ & 0.71 & $35 \%$ \\
& $(0.53-0.79)$ & $(11-58)$ & $(0.61-0.81)$ & $(16-60)$ \\
Parafoveal vd & 0.63 & $30 \%$ & 0.67 & $28 \%$ \\
& $(0.49-0.76)$ & $(11-53)$ & $(0.57-0.77)$ & $(06-47)$ \\
Superior vd & 0.59 & $17 \%$ & 0.65 & $24 \%$ \\
& $(0.45-0.72)$ & $(04-33)$ & $(0.53-0.75)$ & $(06-48)$ \\
Inferior vd & 0.62 & $20 \%$ & 0.71 & $29 \%$ \\
& $(0.48-0.74)$ & $(07-38)$ & $(0.61-0.81)$ & $(08-48)$ \\
\hline
\end{tabular}

AUC: area under the receiver operating characteristic curve; vd: vessel density.

Table 3. Diagnostic ability of structural parameters of optical coherence tomography in differentiating primary open angle glaucoma (POAG) eyes with and without disc hemorrhage (DH) from control eyes (figures in parenthesis represent $95 \%$ confidence intervals).

\begin{tabular}{ccccc}
\hline & \multicolumn{2}{c}{ POAG with DH } & \multicolumn{2}{c}{ POAG without DH } \\
\cline { 2 - 4 } Vessel density & AUC & $\begin{array}{c}\text { Sensitivity at } \\
\mathbf{9 0 \%} \text { specificity }\end{array}$ & AUC & $\begin{array}{c}\text { Sensitivity at 90\% } \\
\text { specificity }\end{array}$ \\
\hline Neuroretinal rim area & 0.93 & $82 \%$ & 0.98 & $91 \%$ \\
& $(0.83-0.98)$ & $(64-97)$ & $(0.91-1.00)$ & $(74-100)$ \\
Peripapillary RNFL thickness & 0.91 & $72 \%$ & 0.95 & $86 \%$ \\
Superotemporal RNFL & $(0.79-0.97)$ & $(47-90)$ & $(0.89-0.99)$ & $(68-97)$ \\
& 0.85 & $67 \%$ & 0.89 & $71 \%$ \\
Inferotemporal RNFL & $(0.72-0.94)$ & $(41-84)$ & $(0.78-0.95)$ & $(46-84)$ \\
& 0.91 & $78 \%$ & 0.90 & $79 \%$ \\
Average GCC thickness & $(0.81-0.97)$ & $(58-94)$ & $(0.80-0.97)$ & $(61-91)$ \\
& 0.83 & $55 \%$ & 0.91 & $78 \%$ \\
Superior GCC thickness & $(0.68-0.91)$ & $(31-74)$ & $(0.83-0.96)$ & $(63-94)$ \\
& 0.76 & $45 \%$ & 0.86 & $62 \%$ \\
Inferior GCC thickness & $(0.61-0.86)$ & $(22-66)$ & $(0.76-0.93)$ & $(39-82)$ \\
\hline
\end{tabular}

AUC: area under the receiver operating characteristic curve; RNFL: retinal nerve fiber layer; GCC: ganglion cell complex.

Figure 2 shows the sensitivity at $90 \%$ specificity of the (a) average peripapillary and (b) parafoveal vessel density measurements at different severities of glaucomatous VF loss in POAG eyes with and without DH. Figure 3 shows the sensitivity at $90 \%$ specificity of the (a) average RNFL and (b) GCC thickness measurements 
at different severities of glaucomatous VF loss in POAG eyes with and without $\mathrm{DH}$. Sensitivities of vessel density and structural measurements increased with increasing severity of glaucoma both in POAG eyes with and without DH. Though the sensitivities of structural parameters seemed to be better in POAG eyes without DH compared to POAG eyes with DH in early stages of glaucoma, the differences were statistically insignificant.
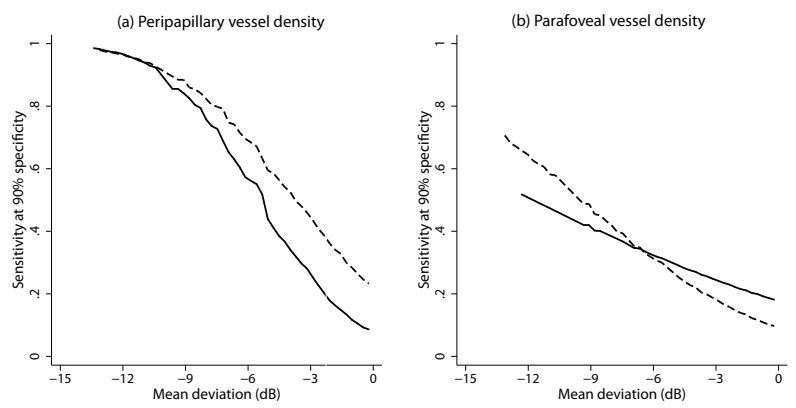

Figure 2 . Sensitivity at $90 \%$ specificity of (a) average peripapillary vessel density and (b) average parafoveal vessel density according to mean deviation on visual fields. Solid lines represent sensitivity in primary open angle glaucoma eyes with disc hemorrhage and dotted line in primary open angle glaucoma eyes without disc hemorrhage.
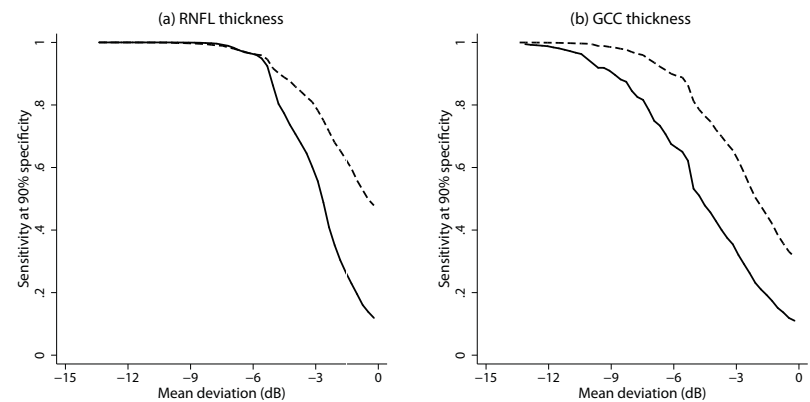

Figure 3. Sensitivity at $90 \%$ specificity of (a) average peripapillary retinal nerve fiber layer (RNFL) thickness and (b) average ganglion cell complex (GCC) thickness according to mean deviation on visual fields. Solid lines represent sensitivity in primary open angle glaucoma eyes with disc hemorrhage and dotted line in primary open angle glaucoma eyes without disc hemorrhage.

The entire analysis also was done considering optic disc and VF abnormality as the definition of glaucoma. The analysis included 26 POAG eyes with DH and 50 age and glaucoma severity matched POAG eyes without $\mathrm{DH}$. Whole enface vessel 
density of disc scan and average peripapillary vessel density showed the best AUCs in POAG eyes with DH ( 0.87 and 0.75 ) and POAG eyes without DH ( 0.93 and 0.85). Rim area and peripapillary RNFL thickness showed the best AUCs in POAG eyes with $\mathrm{DH}(0.94$ and 0.91 ) and POAG eyes without $\mathrm{DH}$ (1.00 and 0.96).

The entire analysis also was done considering one random eye per subject and, optic disc and VF abnormality as the definition of glaucoma. The analysis included 25 POAG eyes with DH (median MD: $-4.1 \mathrm{~dB}$ ) and 33 age and glaucoma severity matched POAG eyes without DH (median MD: $-4.4 \mathrm{~dB}$ ). Whole enface vessel density of disc scan and average peripapillary vessel density showed the best AUCs in POAG eyes with DH ( 0.86 and 0.75 ) and POAG eyes without DH ( 0.93 and 0.86). Rim area and peripapillary RNFL thickness showed the best AUCs in POAG eyes with $\mathrm{DH}$ (0.94 and 0.92) and POAG eyes without DH (1.00 and 0.98).

\section{DISCUSSION}

The present study compares OCTA vessel density measurements in POAG eyes with and without DH. Glaucoma severity, as based on the MD of VF, was matched between the eyes with and without $\mathrm{DH}$. Most of the vessel densities in the $\mathrm{ONH}$, peripapillary and macular regions in POAG eyes with $\mathrm{DH}$ were similar to that in eyes without DH. AUCs and sensitivities at $90 \%$ specificity of vessel density parameters in POAG eyes with DH were statistically similar to that in POAG eyes without $\mathrm{DH}$. These results suggest that vessel density reduction with OCTA in POAG eyes with DH is not greater than that seen in POAG eyes without DH.

Though glaucoma severity, as based on the MD of VF, was matched between the POAG eyes with and without $\mathrm{DH}, \mathrm{ONH}$ rim area, inferonasal RNFL thickness and macular GCC thickness were significantly less in the POAG eyes without $\mathrm{DH}$ compared to those with $\mathrm{DH}$. AUCs and sensitivities at $90 \%$ specificity of structural parameters in POAG eyes without $\mathrm{DH}$ were also greater than that in POAG eyes with $\mathrm{DH}$, though the differences were not statistically significant. We also evaluated the sensitivities of vessel density and structural measurements in 
POAG eyes with and without DH separately across the range of glaucoma severity (Figure 2 and 3 ). Though the sensitivities of structural parameters seemed to be better in POAG eyes without DH compared to POAG eyes with DH in early stages of glaucoma, the differences were statistically insignificant. This most likely shows that the severity of glaucoma, though matched on VFs, was greater in POAG eyes without $\mathrm{DH}$, as noted on the structural measurements. However, glaucoma severity staging systems mostly depend on functional measurements ${ }^{34,35}$ and it is well known that the commonly used structural and functional measurements do not agree well at any given stage of glaucoma. ${ }^{36}$

Major clinical trials have shown $\mathrm{DH}$ to be an important risk factor for the development and progression of glaucoma. ${ }^{5-7}$ Other prospective longitudinal studies have also shown a strong association between $\mathrm{DH}$ and faster rates of structural and functional progression in glaucoma. ${ }^{37-39}$ However, the exact pathogenesis of $\mathrm{DH}$ is still not completely understood. A few studies propose primary vascular abnormality as the cause for $\mathrm{DH}^{9}{ }^{9}, 10$ However, studies evaluating the ocular blood flow in eyes with $\mathrm{DH}$ are sparse. Kurvinen et al used scanning laser Doppler flowmetry to evaluate changes in peripapillary retinal blood flow at the time of detection of $\mathrm{DH}$ and again 6 months later. ${ }^{40}$ They found that the mean, systolic and diastolic flow was decreased at the time of detection of $\mathrm{DH}$ and was significantly increased after $\mathrm{DH}$ resorption. They proposed ischemia at the time of onset of $\mathrm{DH}$ and reperfusion subsequently as the reason for these findings. They also concluded that their results favored the vascular etiology of $\mathrm{DH} .{ }^{40}$ Park et al. used fluorescein angiography to evaluate blood flow in eyes with $\mathrm{DH}^{41}$ They divided the eyes into those with the DH at the border of localized RNFL defects and those with DH location not related to localized RNFL defects. They found that DHs occurring at the margins of RNFL defects had accompanying hemodynamic changes (prolonged arm-retina time and arteriovenous transit time), which were not apparent in DHs not related to RNFL defects. ${ }^{41}$ They suggested that some cases of DH were associated with vascular abnormality while some were not associated with it. A few studies, on the contrary, have proposed mechanical vascular disruption at the level of the lamina cribrosa or the margin of optic disc and RNFL defect as the cause of DH. ${ }^{11,12}$ Recent studies have also provided justification for this by showing a strong association between $\mathrm{DH}$ and focal lamina 
cribrosa defects as well as good spatial concordance between the two. ${ }^{13,14}$ Although there are no studies to date examining the vessel densities using OCTA in eyes with $\mathrm{DH}$, a recent study by Suh et al evaluated the OCTA vessel densities in POAG eyes with and without lamina cribrosa defects. They found that in eyes with similar visual sensitivity loss, the decrease in vessel density in POAG eyes with focal lamina cribrosa defects was significantly greater than in POAG eyes without focal lamina cribrosa defects. ${ }^{42}$ Because of the strong concordance between DH and lamina cribrosa defects, this result would suggest that the decrease in vessel densities in POAG eyes with DH would also be greater than in POAG eyes without $\mathrm{DH}$. Interestingly, however, the prevalence of $\mathrm{DH}$ was similar in the groups with (4/41) and without (7/41) lamina cribrosa defects in their study. ${ }^{42}$

AUCs of structural parameters (rim area, RNFL and GCC thickness) were significantly greater ( $p<0.05$ for all comparisons) than that of the vessel density measurements in the $\mathrm{ONH}$, peripapillary and the macular regions both in POAG eyes with and without $\mathrm{DH}$. This may suggest that structural changes occur before vessel density changes in POAG. On the contrary, this result can be biased. One reason is that the definition of glaucoma was based on the neuroretinal rim and RNFL changes on clinical examination and stereo photographs of the optic discs. This may have biased the diagnostic abilities in favor of the structural measurements. Hence, a separate analysis was performed considering VF changes as the definition of glaucoma and the results were the same. Another possible reason for this can be the effect of anti-glaucoma medications on the vessel density measurements. Though there are no systematic reports evaluating this using OCTA, a previous meta-analysis has reported increased ocular blood flow with topical carbonic anhydrase inhibitors. ${ }^{43}$ It is therefore possible that the anti-glaucoma medications increase the vessel density measurements of OCTA independent of the IOP lowering effect. This can reduce the difference in the vessel density measurements between control and treated POAG eyes and thereby the diagnostic abilities.

There are some limitations of the OCTA technology and the study design which need to be considered while interpreting the results. The OCTA algorithm, in its current form, includes large vessels along with capillaries in its estimation of 
vessel density. The software also does not provide further insights into the nature of vascular changes such as attenuation, drop-out, etc. The technology also does not evaluate the choroidal vasculature. These details would provide a better understanding of the vascular changes in glaucoma. Another possible limitation of the current study was that we did not measure the blood pressure of the subjects or record their anti-hypertensive medication. However, we recorded the history of hypertension and found that the number of subjects with hypertension was similar in the POAG group with and without $\mathrm{DH}$. A previous study also has shown no relationship between blood pressure readings and peripapillary vessel densities on OCTA. ${ }^{20}$ The peripapillary vessel densities can also be affected by parapapillary atrophy (PPA). ${ }^{44}$ We did not record the presence of PPA or its extent in our subjects. We also did not match the groups with respect to the topical anti-glaucoma medication use. Significantly greater number of POAG eyes with $\mathrm{DH}$ were on beta blockers and carbonic anhydrase inhibitors compared to those without $\mathrm{DH}$. Although there are no studies till date evaluating the effect of anti-glaucoma medications on the vessel density measurements of OCTA, some confounding effect of the anti-glaucoma medications on the results cannot be ruled out. The sample size of our study, especially the group of POAG eyes with $\mathrm{DH}$, was small. The power of the study to detect statistically significant vessel density differences between the POAG eyes with and without DH was less than $50 \%$ for most OCTA parameters. Lastly, the follow-up duration of POAG patients was short. Therefore, it is possible that the POAG patients without DH could have had a $\mathrm{DH}$ in the past (when they were not monitored) or during their future visits. Also, DHs could have occurred between study visits and escaped detection. Future studies with longer follow-up are needed to validate our results.

In conclusion, there was no difference in the OCTA measured vessel densities in the $\mathrm{ONH}$, peripapillary and macular regions in POAG eyes with $\mathrm{DH}$ compared to POAG eyes without DH, when the severity of glaucoma was matched for. This suggests that the cause of $\mathrm{DH}$ is more likely to be mechanical vascular disruption rather than a primary vascular abnormality. 


\section{REFERENCES}

1. Weinreb RN, Khaw PT. Primary open-angle glaucoma. Lancet 2004;363:1711-20.

2. Weinreb RN, Aung T, Medeiros FA. The pathophysiology and treatment of glaucoma: a review. JAMA 2014;311:1901-11.

3. Bonomi L, Marchini G, Marraffa M, et al. Vascular risk factors for primary open angle glaucoma: the Egna-Neumarkt Study. Ophthalmology 2000;107:1287-93.

4. Leske MC, Heijl A, Hyman L, et al. Predictors of long-term progression in the Early Manifest Glaucoma Trial. Ophthalmology 2007;114:1965-72.

5. Budenz DL, Anderson DR, Feuer WJ, et al. Detection and prognostic significance of optic disc hemorrhages during the Ocular Hypertension Treatment Study. Ophthalmology 2006;113:2137-43.

6. Bengtsson B, Leske MC, Yang Z, et al. Disc hemorrhages and treatment in the Early Manifest Glaucoma Trial. Ophthalmology 2008;115:2044-8.

7. Drance S, Anderson DR, Schulzer M, Collaborative Normal-Tension Glaucoma Study G. Risk factors for progression of visual field abnormalities in normal-tension glaucoma. Am J Ophthalmol 2001;131:699-708.

8. Ernest PJ, Schouten JS, Beckers HJ, et al. An evidence-based review of prognostic factors for glaucomatous visual field progression. Ophthalmology 2013;120:512-9.

9. Shim SH, Kim JM, Woo HY, et al. Association Between Platelet Function and Disc Hemorrhage in Patients With Normal-Tension Glaucoma: A Prospective Cross-Sectional Study. Am J Ophthalmol 2015;160:1191-9.

10. Park HY, Park SH, Oh YS, Park CK. Nail bed hemorrhage: a clinical marker of optic disc hemorrhage in patients with glaucoma. Arch Ophthalmol 2011;129:1299-304.

11. Quigley HA, Addicks EM, Green WR, Maumenee AE. Optic nerve damage in human glaucoma. II. The site of injury and susceptibility to damage. Arch Ophthalmol 1981;99:635-49.

12. Nitta K, Sugiyama K, Higashide T, et al. Does the enlargement of retinal nerve fiber layer defects relate to disc hemorrhage or progressive visual field loss in normal-tension glaucoma? J Glaucoma 2011;20:189-95.

13. Kim YK, Jeoung JW, Park KH. Effect of Focal Lamina Cribrosa Defect on Disc Hemorrhage Area in Glaucoma. Invest Ophthalmol Vis Sci 2016;57:899-907.

14. Sharpe GP, Danthurebandara VM, Vianna JR, et al. Optic Disc Hemorrhages and Laminar Disinsertions in Glaucoma. Ophthalmology 2016;123:1949-56.

15. Jia Y, Tan O, Tokayer J, et al. Split-spectrum amplitude-decorrelation angiography with optical coherence tomography. Opt Express 2012;20:4710-25.

16. Jia Y, Morrison JC, Tokayer J, et al. Quantitative OCT angiography of optic nerve head blood flow. Biomed Opt Express 2012;3:3127-37.

17. Jia $Y$, Wei $E$, Wang $X$, et al. Optical coherence tomography angiography of optic disc perfusion in glaucoma. Ophthalmology 2014;121:1322-32.

18. Wang $\mathrm{X}$, Jiang $\mathrm{C}, \mathrm{Ko} \mathrm{T}$, et al. Correlation between optic disc perfusion and glaucomatous 
severity in patients with open-angle glaucoma: an optical coherence tomography angiography study. Graefes Arch Clin Exp Ophthalmol 2015;253:1557-64.

19. Leveque PM, Zeboulon P, Brasnu E, et al. Optic Disc Vascularization in Glaucoma: Value of Spectral-Domain Optical Coherence Tomography Angiography. J Ophthalmol 2016;2016:6956717.

20. Liu L, Jia Y, Takusagawa HL, et al. Optical Coherence Tomography Angiography of the Peripapillary Retina in Glaucoma. JAMA Ophthalmol 2015;133:1045-52.

21. Yarmohammadi A, Zangwill LM, Diniz-Filho A, et al. Optical Coherence Tomography Angiography Vessel Density in Healthy, Glaucoma Suspect, and Glaucoma Eyes. Invest Ophthalmol Vis Sci 2016;57:OCT451-9.

22. Rao HL, Pradhan ZS, Weinreb RN, et al. Regional Comparisons of Optical Coherence Tomography Angiography Vessel Density in Primary Open-Angle Glaucoma. Am J Ophthalmol 2016;171:75-83.

23. Kraus MF, Potsaid B, Mayer MA, et al. Motion correction in optical coherence tomography volumes on a per A-scan basis using orthogonal scan patterns. Biomed Opt Express 2012;3:1182-99.

24. Rao HL, Zangwill LM, Weinreb RN, et al. Comparison of different spectral domain optical coherence tomography scanning areas for glaucoma diagnosis. Ophthalmology 2010;117:1692-9.

25. Rao HL, Leite MT, Weinreb RN, et al. Effect of disease severity and optic disc size on diagnostic accuracy of RTVue spectral domain optical coherence tomograph in glaucoma. Invest Ophthalmol Vis Sci 2011;52:1290-6.

26. Glynn RJ, Rosner B. Accounting for the correlation between fellow eyes in regression analysis. Arch Ophthalmol 1992;110:381-7.

27. Zhou XH, Obuchowski NA, McClish DK. Analysis of correlated ROC data. In: Zhou XH, Obuchowski NA, McClish DK, eds. Statistical Methods in Diagnostic Medicine. New York:John Wiley \& Sons, Inc.;2002:274-306.

28. Alonzo TA, Pepe MS. Distribution-free ROC analysis using binary regression techniques. Biostatistics 2002;3:421-32.

29. Zhou XH, Obuchowski NA, McClish DK Comparing the accuracy of two diagnostic tests. In: Zhou XH, Obuchowski NA, McClish DK, eds. Statistical Methods in Diagnostic Medicine. New York:John Wiley \& Sons; 2002:165-94.

30. DeLong ER, DeLong DM, Clarke-Pearson DL. Comparing the areas under two or more correlated receiver operating characteristic curves: a nonparametric approach. Biometrics 1988;44:837-45.

31. Pepe MS. Three approaches to regression analysis of receiver operating characteristic curves for continuous test results. Biometrics 1998;54:124-35.

32. Pepe MS. An interpretation for the ROC curve and inference using GLM procedures. Biometrics 2000;56:352-9.

33. Pepe MS. The Statistical Evaluation of Medical Tests for Classification and Prediction. Oxford, UK: Oxford University Press; 2003:130-67. 


\section{Chapter 9}

34. Hodapp E, Parrish RK, Anderson DR, ed. Clinical decisions in glaucoma. St. Louis:Mosby;1993:53.

35. Brusini P, Filacorda S. Enhanced Glaucoma Staging System (GSS 2) for classifying functional damage in glaucoma. J Glaucoma 2006;15:40-6.

36. Hood DC, Kardon RH. A framework for comparing structural and functional measures of glaucomatous damage. Prog Retin Eye Res 2007;26:688-710.

37. De Moraes CG, Prata TS, Liebmann CA, et al. Spatially consistent, localized visual field loss before and after disc hemorrhage. Invest Ophthalmol Vis Sci 2009;50:4727-33.

38. Medeiros FA, Alencar LM, Sample PA, et al. The relationship between intraocular pressure reduction and rates of progressive visual field loss in eyes with optic disc hemorrhage. Ophthalmology 2010;117:2061-6.

39. Gracitelli CP, Tatham AJ, Zangwill LM, et al. Estimated rates of retinal ganglion cell loss in glaucomatous eyes with and without optic disc hemorrhages. PLoS One 2014;9:e105611.

40. Kurvinen L, Harju M, Saari J, Vesti E. Altered temporal peripapillary retinal flow in patients with disc hemorrhages. Graefes Arch Clin Exp Ophthalmol 2010;248:1771-5.

41. Park HY, Jeong HJ, Kim YH, Park CK. Optic disc hemorrhage is related to various hemodynamic findings by disc angiography. PLoS One 2015;10:e0120000.

42. Suh MH, Zangwill LM, Manalastas PI, et al. Optical Coherence Tomography Angiography Vessel Density in Glaucomatous Eyes with Focal Lamina Cribrosa Defects. Ophthalmology 2016;123:2309-17.

43. Siesky B, Harris A, Brizendine E, et al. Literature review and meta-analysis of topical carbonic anhydrase inhibitors and ocular blood flow. Surv Ophthalmol 2009;54:33-46.

44. Akagi T, lida Y, Nakanishi H, et al. Microvascular Density in Glaucomatous Eyes With Hemifield Visual Field Defects: An Optical Coherence Tomography Angiography Study. Am J Ophthalmol 2016;168:237-49.

45. Kitazawa Y, Shirato S, Yamamoto T. Optic disc hemorrhage in low-tension glaucoma. Ophthalmology 1986;93:853-7. 

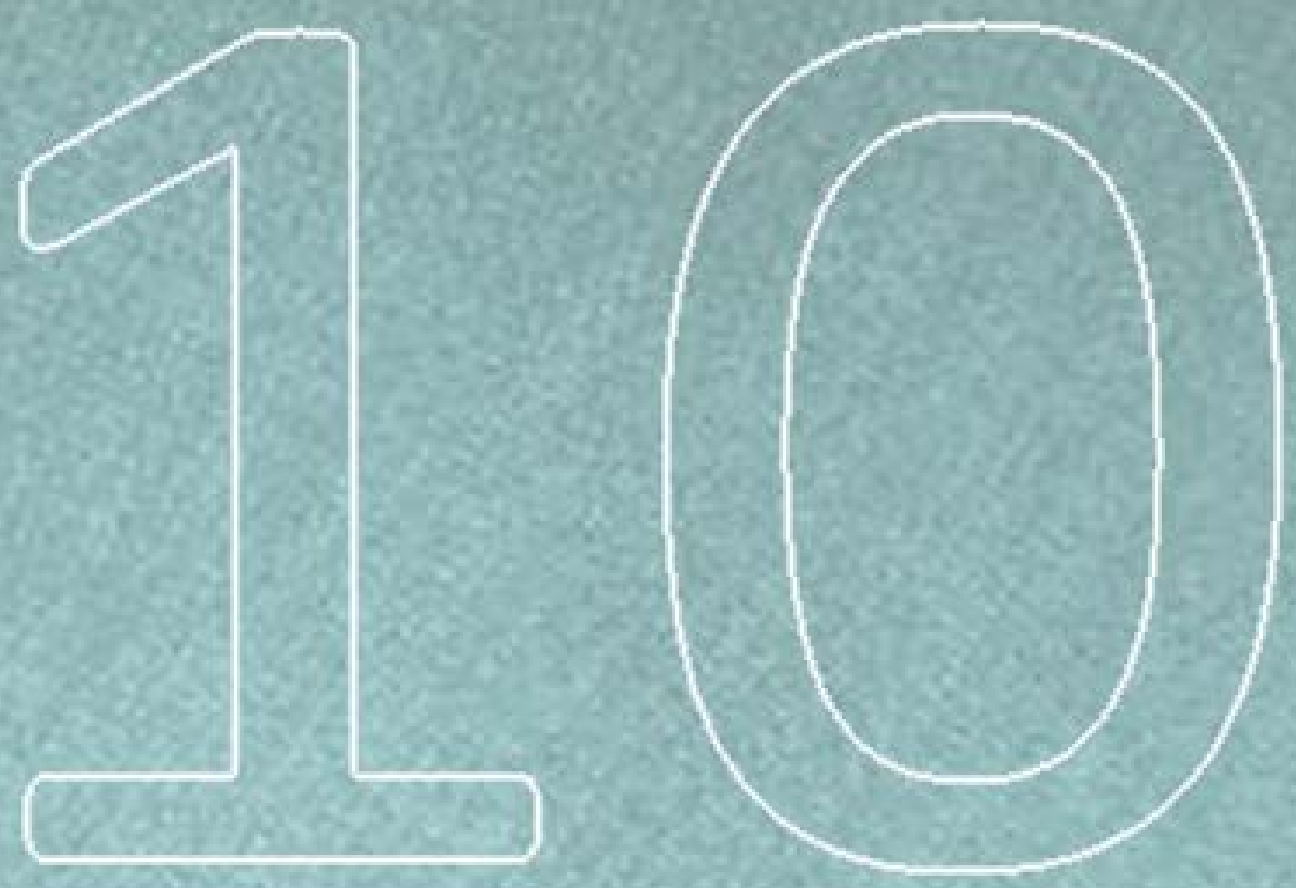

Discussion 
Discussion 


\section{GENERAL DISCUSSION}

Glaucoma is the leading cause of irreversible blindness in the world. The exact pathogenesis of glaucoma, however, is not fully understood. Mechanical (intraocular pressure [IOP] related mechanical damage) and vascular (ischemic damage due to reduced blood supply) theories are the two commonly proposed theories to explain its pathogenesis.

The diagnosis of glaucoma in current day practice is based on the assessment of structural and functional damage at the level of the optic nerve head (ONH). Evaluating the structural damage on clinical examination (optic nerve examination on slit lamp biomicroscopy using a high powered convex lens), stereoscopic optic disc photographs and / or optical coherence tomography (OCT ONH rim area, retinal nerve fiber layer [RNFL] thickness and ganglion cell complex [GCC] thickness) are the standard methods employed to diagnose glaucoma. Similarly, assessing the functional damage on standard automated perimetry is the standard method employed in clinical practice. However, unlike structural and functional methods, there are no validated methods to quantify the vascular changes in glaucoma. Available methods to assess vascular changes were either invasive, poorly repeatable or not quantifiable and are therefore rarely used in clinical practice. Recently developed OCT angiography (OCTA) has generated hopes of filling this void in glaucoma.

The primary purpose of this thesis was to revisit the vascular theory of glaucoma using OCTA. The thesis also provided an in-depth evaluation of the clinical utility of OCTA in the most common types of glaucoma seen in clinical practice. Most of the work was conducted in primary open angle glaucoma (POAG), both highpressure $O A G$ and normal-pressure $O A G$, and in primary angle closure glaucoma (PACG) subjects.

Our work found that the OCTA-measured vessel densities within the ONH, in the peripapillary and macular regions were significantly lesser in glaucoma eyes compared to control eyes (chapters 4-7). Our work also showed that the OCTAmeasured vessel densities were repeatable (Chapter 2 ). This demonstrated the 
presence of vessel density loss in glaucoma eyes and that OCTA was a useful tool to precisely quantify the amount of decrease in vessel density.

Next we evaluated if the vessel density decrease in glaucoma was a primary event or a secondary event (secondary to the death of retinal ganglion cells). For this, we compared the diagnostic ability of OCTA-measured vessel density with that of the OCT-measured structural parameters (ONH rim area, RNFL and GCC thickness) and found that the diagnostic abilities of structural measurements were better than that of the vessel density measurements both in POAG and PACG (Chapters 5 and 7). We further evaluated the question in primary angle closure (PAC) eyes, which had high IOP at presentation but normal optic nerves. We found significant thinning of a few structural measurements in PAC eyes compared to control eyes but found no difference in the vessel density measurements. This demonstrated that high IOP affects the structural measurements earlier than the vessel density measurements. All these findings indirectly point to the fact that the decrease in OCTA-measured vessel density seen in glaucoma eyes is secondary to structural damage and not a primary event.

It is generally accepted that the vascular theory of glaucoma is more relevant in normal-pressure OAG (normal tension glaucoma [NTG] / normal pressure glaucoma [NPG] / low tension glaucoma [LTG]) than high-pressure OAG. To address this, we evaluated the effect of pre-treatment (baseline) IOP on the diagnostic ability of vessel density measurements in glaucoma (Chapters 4 and 5), after controlling for the disease severity. We found that the pre-treatment IOP was significantly associated only with the diagnostic ability of vessel density within the $\mathrm{ONH}$ but not with either the peripapillary or the macular vessel densities. This demonstrated that the OCTA-measured vessel densities decrease was similar both in high-pressure OAG and NTG. In the same context, we also evaluated the diagnostic ability of vessel density measurements in POAG with disc hemorrhage and compared the same in POAG eyes without disc hemorrhage (Chapter 9). Disc hemorrhage is known to be more common in eyes with NTG. We once again found that the diagnostic ability of vessel density measurements was similar in POAG eyes with and without disc hemorrhage. This also demonstrated that the etiology of disc hemorrhage was unlikely to be a vascular abnormality or that the OCTA 
was not the best technique to investigate this.

Most of work related to the thesis was conducted in a cross-sectional study design. Conclusive answers to all the above questions need to come from longitudinal studies. Though this is a limitation, the findings of our thesis give future directions to longitudinal studies with OCTA. The test-retest repeatability of OCTA measurements (Chapter 2), though better than that of the previous technologies (laser Doppler flowmetry, laser speckle flowgraphy), was clinically large. This has to be considered while evaluating longitudinal changes in vessel densities for clinically significant change. OCTA-measured vessel densities also were affected by multiple eye-related, subject-related and OCT technology related factors (Chapter 3). Most of the peripapillary vessel densities were lower, while the parafoveal vessel density was higher in subjects with hypertension. Most of the vessel densities were lower in subjects with diabetes (Chapter 3). Signal strength of the OCTA scans was found to be consistently associated with the vessel density measurements and their repeatability (Chapter 2, 3 and 10). Increase in signal strength was associated with higher vessel density measurements. These determinants should also be considered while evaluating vessel density measurements longitudinally for detecting glaucoma progression. In addition to the factors which we evaluated in the thesis, there could be multiple other factors that may affect the OCTA measurements. For example, medications taken for systemic conditions as well as topical anti-glaucoma medications can affect the OCTA measurements. We however did not evaluate the effect of systemic and topical medications on the OCTA measurements in our thesis. There is a need for future studies to evaluate this.

We also noticed some of the limitations of OCTA during the course of the thesis. A significant proportion of scans obtained for this research was found to be of poor quality, primarily due to motion artifacts. This may limit the clinical utility of OCTA imaging in day to day practice. The new generation of devices performing OCTA, therefore, come with an eye-tracker so as to nullify the effect of eye movements during scanning. Another limitation of the OCTA software is that both the large vessels and capillaries are considered together during quantification. It is quite possible that the vascular changes in glaucoma may be more pronounced in 
capillaries compared to large vessels, or vice-versa. Therefore, there is a need to evaluate large vessel and capillary changes separately in glaucoma. Also, current OCTA technology is not able to evaluate the choroidal vasculature accurately due to projection artifacts; superficial vessels casting a shadow in the choroidal angiography slabs. These details are important to provide a better understanding of the vascular changes in glaucoma.

In conclusion, this thesis gives a detailed knowledge on the utility of OCTA in current day glaucoma practice and provides insights into certain aspects of the vascular theory of glaucoma evaluated using the OCTA. 

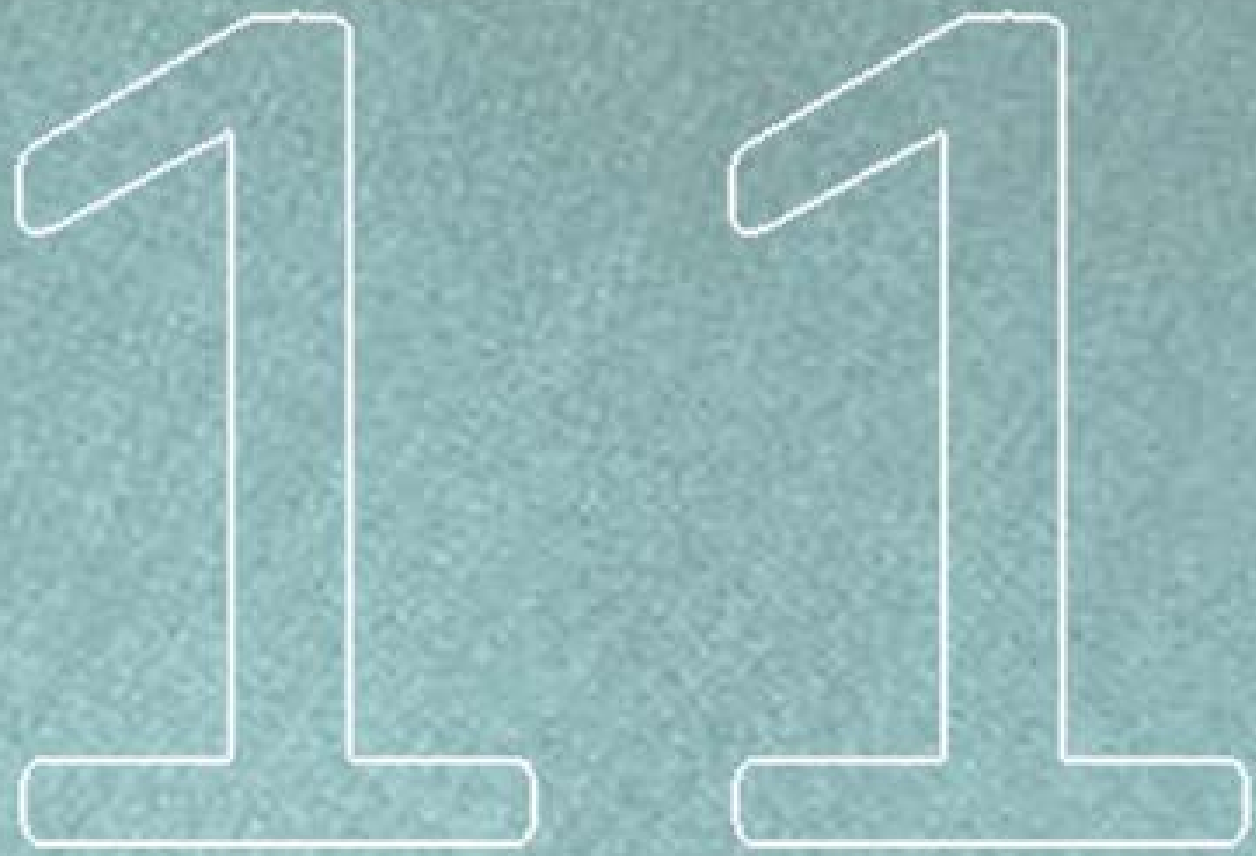

\section{Valorization addendum}




\section{Relevance of this research}

Glaucoma is the leading cause of irreversible blindness world-over, as well as in my country, India. Based on the results of the population based studies conducted in different parts of India till now, ${ }^{1-5}$ George et al ${ }^{6}$ reported the burden of glaucoma in India to be 11.2 million. Of these, primary open angle glaucoma (POAG) was estimated to be 6.48 million and primary angle closure glaucoma (PACG) was 2.54 million. If we include primary angle closure (PAC) along with PACG, with the idea of estimating the number of people with primary angle closure disease (PACD) that require treatment, the number is 6.6 million, similar to that of POAG. ${ }^{6}$ The clinical course and severity of primary glaucoma is not uniform across populations. Angle closure glaucoma in India is different from that seen in other parts of South-east Asia. PACD tends to have a more chronic course and acute angle closures are relatively rare. ${ }^{7}$ The burden of glaucoma blindness in India is 1.1 million. PACG on an average produced 2 times the proportion of bilateral blindness than POAG in India. ${ }^{6}$ Glaucoma, a disease with such a significant burden on the community, is of great societal significance. Studying the utility of optical coherence tomograpy angiography (OCTA) in PACD, a disease with a high prevalence in India, is of significant relevance to us.

In spite of glaucoma being such a significant disease, the exact pathogenesis of it is not fully understood. Mechanical (IOP related mechanical damage) and vascular (ischemic damage due to reduced blood supply) theories are the two commonly proposed theories to explain its pathogenesis. However, the vascular theory is not studied well because of a lack of simple, non-invasive, accurate and precise method for studying ocular blood flow. Optical coherence tomography angiography (OCTA) came with the hope of fulfilling most of the requirements for an ideal test to evaluate ocular blood flow. Evaluating OCTA therefore was necessary to determine if it was a useful test to quantify the ocular blood supply and to contribute to the pathogenesis of glaucoma.

Two crucial steps in the management of glaucoma are to diagnose the disease early and to prevent disease progression by effective treatment. Early detection 
of glaucoma has received great emphasis as the optic nerve damage from glaucoma remains irreversible till date. Evaluating rim area, RNFL thickness and GCC thickness on OCT is one of the preferred method to diagnose glaucoma early. We sought out to evaluate if the OCTA-measured vessel density was able to diagnose glaucoma earlier than the structural measurements of OCT. If so, this could lead to a paradigm shift in the way glaucoma is diagnosed and monitored. In our cross-sectional analysis, we however found that the OCTA-measured vessel densities were inferior to OCT-measured structural parameters in diagnosing glaucoma in early stages.

The primary treatment of glaucoma still revolves around managing the intraocular pressure (IOP) and IOP still remains a primary outcome measure in evaluating the efficacy of various treatments. If OCTA is useful in evaluating the vascular changes in glaucoma, this could possibly open up new ways of evaluating treatment outcomes.

\section{Target groups}

Beneficiaries of the results of this dissertation are the clinicians, academic researchers and the industry. The essential theme of the entire thesis was to evaluate the clinical utility of OCTA in glaucoma. The results therefore are straight away applicable to all clinicians. The take home message from the thesis for the clinicians is that OCTA evaluates the blood vessels of the superficial retina precisely. However, the technology still is not equivalent to traditional OCTmeasured structural parameters in diagnosing glaucoma and so the technology in its current form is not an essential part of the clinical workup of glaucoma diagnosis. The thesis is also applicable to academic researchers as the results not only give a comprehensive knowledge about OCTA but also gives directions to future research with this technology. For researchers evaluating OCTA in longitudinal studies, the results of the thesis give clear indications of the testretest variability and the factors other than glaucoma progression that can affect a measurement change. For the industry, the thesis provides a factual update 
on the current status of the technology. It demonstrates that there is a definite need for improvements in the technology before it becomes a part of the day to day glaucoma care.

\section{Product}

Translating the results of the thesis to meaningful products started early in the course of the research work. The challenges in the currently available quantification algorithms of OCTA have been mentioned in the earlier chapters. Briefly, one of the challenges is that the software considers both the large vessels and capillaries together during quantification. It is quite possible that the vascular changes in glaucoma may be more pronounced in large vessels compared to capillaries, or vice-versa. There is a need to evaluate large vessel and capillary changes separately in glaucoma. We had done some work in this regard and had used fractal analysis based algorithm to quantify the large and small vessels of retina separately. ${ }^{8}$ This is an ongoing work. The other important challenge is that the OCTA technology is now available on the OCT devices of multiple manufacturers. Cirrus HD-OCT 5000 (Zeiss Meditec, Inc., Dublin, CA, USA) and Spectralis (Heidelberg Engineering, Heidelberg, Germany) are two SDOCT-based devices that perform angiography. Triton (Topcon, Tokyo, Japan) is a swept-source OCT-based device that performs angiography. However, none of these, like Avanti RTVue-XR (Optovue Inc., Fremont, CA, USA), the device used in the thesis, have commercially available quantification software. We have therefore developed a vessel density analysis software that can be used on the images of any of these devices (device-independent software). This is called "ReVeal" (retinal vessel evaluation algorithm). The software is in the testing phase and would be available to the market soon.

\section{Innovativeness of the research}

The innovativeness of the thesis is the fact that the research work was conducted 
keeping the clinical perspective in mind so that the results could be translated directly to the clinics. The other most important aspect was the testing of the technology in angle closure glaucoma, which to the best of our knowledge, is not done before. Angle closure glaucoma is a significant problem in India and the results of our study provide useful information about the utility of OCTA in Indian population.

\section{Realization}

Steps to take the research work of this thesis further is already underway. The results of the thesis demonstrate that the OCTA technology needs to mature a lot before it can become an essential part of the glaucoma workup. A significant proportion of scans obtained for this research was of poor quality, primarily due to motion artifacts. The new generation of devices performing OCTA therefore come with an eye-tracker so as to nullify the effect of eye movements during scanning. Our work in underway to find out if the repeatability and utility of OCTA improves with the introduction of this eye-tracking technology. The studies related to the current thesis are predominantly cross-sectional in design. Longitudinal studies to validate the results of these studies are also ongoing. One of the primary question that remained unanswered in the thesis was the effect of glaucoma drugs on the OCTA-measured vessel densities. Studies are also underway to evaluate this.

\section{REFERENCES}

1. Jacob A, Thomas R, Koshi SP, et al. Prevalence of primary glaucoma in an urban south Indian population. Indian J Ophthalmol 1998;46:81-6.

2. Ramakrishnan R, Nirmalan PK, Krishnadas R, et al. Glaucoma in a rural population of southern India: the Aravind comprehensive eye survey. Ophthalmology 2003;110:1484-90.

3. Vijaya L, George R, Baskaran M, et al. Prevalence of primary open-angle glaucoma in an urban south Indian population and comparison with a rural population. The Chennai Glaucoma Study. Ophthalmology 2008;115:648-54.

4. Vijaya L, George R, Arvind $\mathrm{H}$, et al. Prevalence of primary angle-closure disease in an urban south Indian population and comparison with a rural population. The Chennai Glaucoma Study. Ophthalmology 2008;115:655-60. 


\section{Chapter 11}

5. Garudadri C, Senthil S, Khanna RC, et al. Prevalence and risk factors for primary glaucomas in adult urban and rural populations in the Andhra Pradesh Eye Disease Study. Ophthalmology 2010;117:1352-9.

6. George R, Ve RS, Vijaya L. Glaucoma in India: estimated burden of disease. J Glaucoma 2010;19:391-7.

7. Seah SK, Foster PJ, Chew PT, et al. Incidence of acute primary angle-closure glaucoma in Singapore. An island-wide survey. Arch Ophthalmol 1997;115:1436-40.

8. Kumar RS, Anegondi N, Chandapura RS, et al. Discriminant Function of Optical Coherence Tomography Angiography to Determine Disease Severity in Glaucoma. Invest Ophthalmol Vis Sci 2016;57:6079-88. 

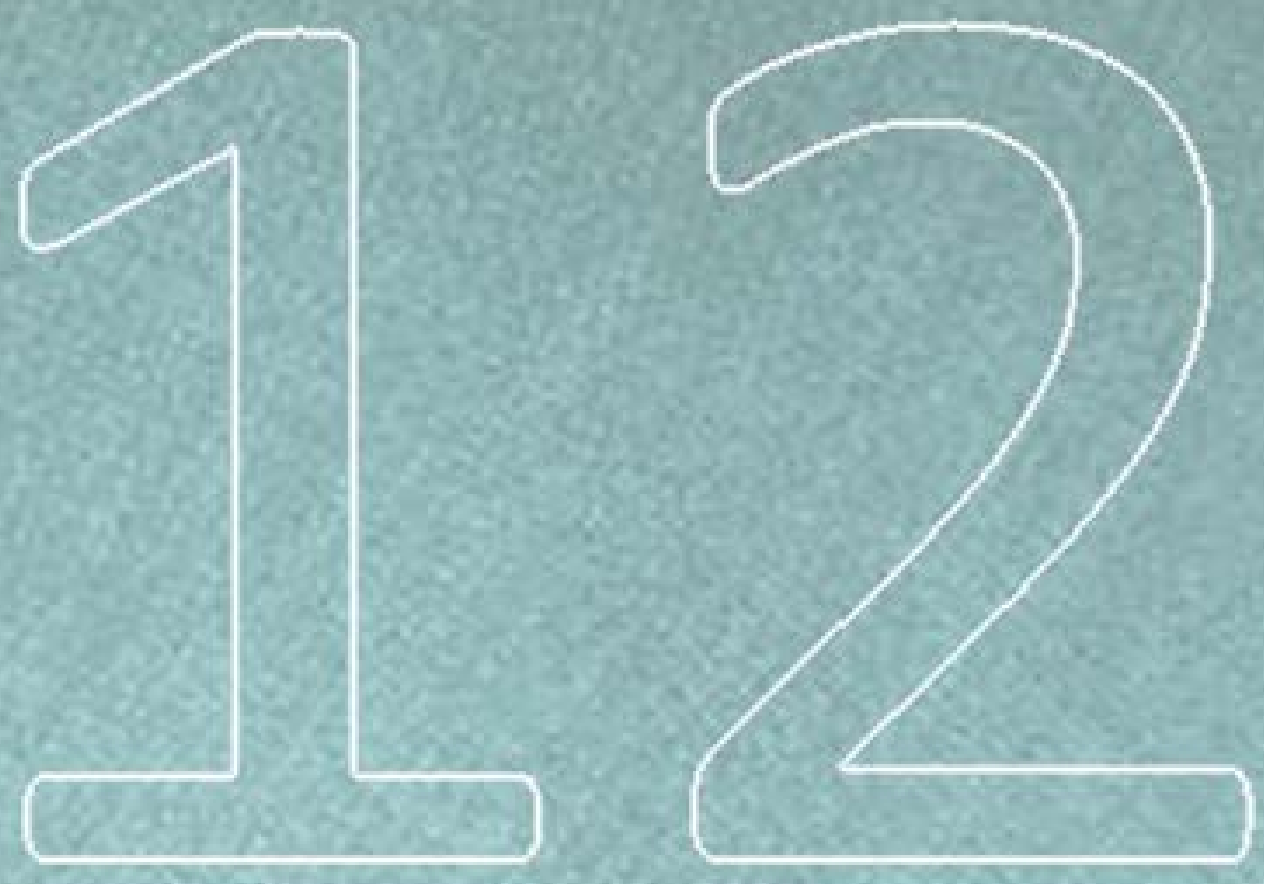

Summary 
Although glaucoma is the leading cause of irreversible blindness in the world, the exact pathogenesis of it is not fully understood. Mechanical (intraocular pressure, IOP, related mechanical damage) and vascular (ischemic damage due to reduced blood supply) theories are the two commonly proposed theories to explain its pathogenesis. The primary purpose of this thesis was to revisit the vascular theory of glaucoma using optical coherence tomography angiography (OCTA). The thesis also provided an in-depth evaluation of the clinical utility of OCTA in the most common types of glaucoma seen in clinical practice. Most of the work here was conducted in primary open angle glaucoma (POAG), which included both high-pressure OAG and normal-pressure OAG, and in primary angle closure glaucoma (PACG) subjects.

In Chapter 2, the intra-session repeatability of vessel density measurements of OCTA was evaluated in normal eyes and eyes with glaucoma separately and the effect of signal strength index (SSI) of OCTA scans on the repeatability was also evaluated. Three optic nerve head (ONH) scans each of 65 eyes (30 normal, 35 glaucoma eyes) and 3 macular scans each of 69 eyes ( 35 normal, 34 glaucoma eyes) acquired in the same session with OCTA were analysed. Repeatability estimates of most vessel density measurements were similar in normal and glaucoma eyes. Vessel densities of both peripapillary and macular regions significantly increased with increase in SSI of repeat scans. Knowing the test-retest variability is important to decide the change in vessel density measurements that can be considered clinically significant. This chapter therefore highlights the change in vessel densities over follow-up that can be considered significant and also highlights the importance of incorporating the change in signal strength of the scans while interpreting the change in vessel density measurements.

In Chapter 3, the effect of subject-related (age, gender, systemic hypertension and diabetes), eye-related (refractive error, optic disc size) and technology-related (SSI of the scans) determinants on the peripapillary and macular vessel densities in normal eyes were evaluated. One hundred and eighty-one normal eyes of 107 subjects (45 men, 62 women, median age: 50 years, range: 18-77 years) underwent OCTA imaging. We found that age and optic disc size did not affect the vessel densities of any of the regions. Most of the peripapillary vessel densities 
were higher in females. Most of the peripapillary vessel densities were lower, while the parafoveal vessel density was higher, in subjects with hypertension. Most of the vessel densities were lower in subjects with diabetes. SSI showed a statistically significant positive association with the vessel densities of all regions. These results should be considered while interpreting the vessel densities in retinal diseases and glaucoma.

In Chapter 4, the diagnostic ability of the OCTA-measured vessel densities within the $\mathrm{ONH}$, in the peripapillary and macular regions was evaluated. Also, the effect of the covariates, such as disease severity and baseline IOP (pre-treatment IOP) on the diagnostic abilities of vessel densities were evaluated. Seventy-eight eyes of 53 control subjects and 64 eyes of 39 POAG patients underwent OCTA imaging. The area under the receiver operating characteristic curves (AUC) of ONH vessel densities ranged between 0.59 (superonasal sector) and 0.73 (average inside disc), peripapillary between 0.70 (nasal, superonasal and temporal) and 0.89 (inferotemporal), and macular between 0.56 (nasal) and 0.64 (temporal). AUC of the average peripapillary vessel density was significantly better than the average inside disc $(p=0.05)$ and macular $(p=0.005)$ measurement. Diagnostic abilities of vessel densities increased with increasing severity of glaucoma and that of $\mathrm{ONH}$ vessel density with higher pre-treatment IOPs.

In Chapter 5, the diagnostic abilities of the OCTA-measured vessel densities within the $\mathrm{ONH}$, in the peripapillary and macular regions in eyes with POAG were compared with that of the OCT-measured neuroretinal rim area, retinal nerve fiber layer (RNFL) thickness and macular ganglion cell complex (GCC) thickness measurements respectively. Seventy-eight eyes of 50 control subjects and 117 eyes of 67 POAG patients underwent vessel density and structural measurements with spectral domain OCT. The AUC of average vessel densities within the ONH, peripapillary and macular region were $0.77,0.85$ and 0.70 respectively. The same of ONH rim area, average RNFL and GCC thickness were 0.94, 0.95 and 0.93 respectively. AUCs of vessel densities were significantly lower $(p<0.05)$ than that of the corresponding structural measurements.

In Chapter 6, the diagnostic abilities of OCTA-measured peripapillary vessel density 
were evaluated in eyes with POAG and primary angle closure glaucoma (PACG). Also, the diagnostic abilities of peripapillary vessel densities were compared with RNFL thickness measurements separately in POAG and PACG. Forty-eight eyes of 33 healthy control subjects, 63 eyes of 39 POAG patients and 49 eyes of 32 PACG patients underwent OCTA and RNFL imaging with spectral domain OCT. AUCs of peripapillary vessel density ranged between 0.48 for the temporal sector and 0.88 for inferotemporal sector in POAG. The same in PACG ranged between 0.57 and 0.86 . AUCs of all peripapillary vessel density measurements were comparable ( $p>0.05$ ) to the corresponding RNFL thickness measurements in both POAG and PACG.

In Chapter 7, a more detailed evaluation of the diagnostic abilities OCTA-measured vessel densities within the $\mathrm{ONH}$ and in the peripapillary and macular regions in eyes with primary angle closure (PAC, high IOP but normal optic disc and visual field) and PACG was conducted. The diagnostic abilities were compared against those of the rim area, RNFL thickness and GCC thickness measurements. Seventyseven eyes of 50 control subjects, 65 eyes of 45 patients with PACG, and 31 eyes of 22 PAC patients with a history of high IOP, underwent imaging with OCT. All the vessel density and structural measurements were significantly lower in the PACG compared to the control group. Vessel densities in the PAC were similar to that of the controls; the superotemporal RNFL, however, was significantly thinner in the PAC group (127 $\mu \mathrm{m}$ vs. $135 \mu \mathrm{m}, \mathrm{p}=0.01)$. The AUC and sensitivity at $95 \%$ specificity of vessel densities within the $\mathrm{ONH}(0.76 \& 42 \%)$ and macular region $(0.69 \& 18 \%)$ in PACG were significantly lower than ONH rim area $(0.90 \& 77 \%)$ and GCC thickness $(0.91 \& 55 \%)$ respectively. AUC and sensitivity of peripapillary vessel density (0.85 \& 53\%) were statistically similar to RNFL thickness (0.91 \& $65 \%)$. These results suggest that structural changes in PACG occur earlier than the reduction in retinal vessel densities.

In Chapter 8, the sectoral and global structure-structure association between OCTA-measured peripapillary vessel density and RNFL thickness, and structurefunction association between peripapillary vessel density and visual sensitivity loss on perimetry in POAG eyes were evaluated. We also evaluated if fractional polynomial (FP) models characterize the relationships better than linear models. 
Structure-structure and structure-function relationships of peripapillary vessel densities were determined in 227 eyes of 143 subjects (63 control and 164 POAG eyes). We found that the $\mathrm{R}^{2}$ values for structure-structure associations using linear models ( 0.53 for superotemporal sector, 0.61 for inferotemporal and 0.53 for average measurements) were statistically significantly lesser than that determined using FP models $\left(0.57,0.65\right.$ and 0.55 respectively). $R^{2}$ values for structure-function associations using linear models ( 0.35 for superotemporal vessel density-inferotemporal visual sensitivity loss, 0.49 for inferotemporal vessel density-superotemporal visual sensitivity loss and 0.39 for average vessel density-average visual sensitivity loss) also were significantly lesser than that determined using FP models $(0.43,0.58$ and 0.47 respectively). This demonstrated that FP models were significantly better than linear models in describing these relationships.

In Chapter 9, the measurements of OCTA-derived vessel densities in POAG eyes with disc hemorrhage (DH) were compared with that of severity-matched POAG eyes without DH. Sixty-six eyes of 46 control subjects, 34 eyes of 33 POAG patients with $\mathrm{DH}$ (median mean deviation, MD: $-3.7 \mathrm{~dB}$ ) and 63 eyes of 43 POAG patients without DH (median MD: $-3.8 \mathrm{~dB}$ ) underwent imaging with spectral domain OCT. Most of the vessel density and structural measurements were similar $(p>0.05)$ in POAG eyes with and without DH. Whole enface vessel density of the disc scan and inferotemporal peripapillary vessel density showed the best AUC and sensitivity at $90 \%$ specificity both in POAG eyes with DH $(0.82,56 \%$ and 0.75 , $59 \%)$ and without $\mathrm{DH}(0.91,73 \%$ and $0.83,67 \%)$. AUCs and sensitivities of vessel density and structural measurements of POAG eyes with and without DH were statistically similar ( $p>0.05)$. This suggests that the cause of DH in POAG is unlikely to be vascular abnormality. 

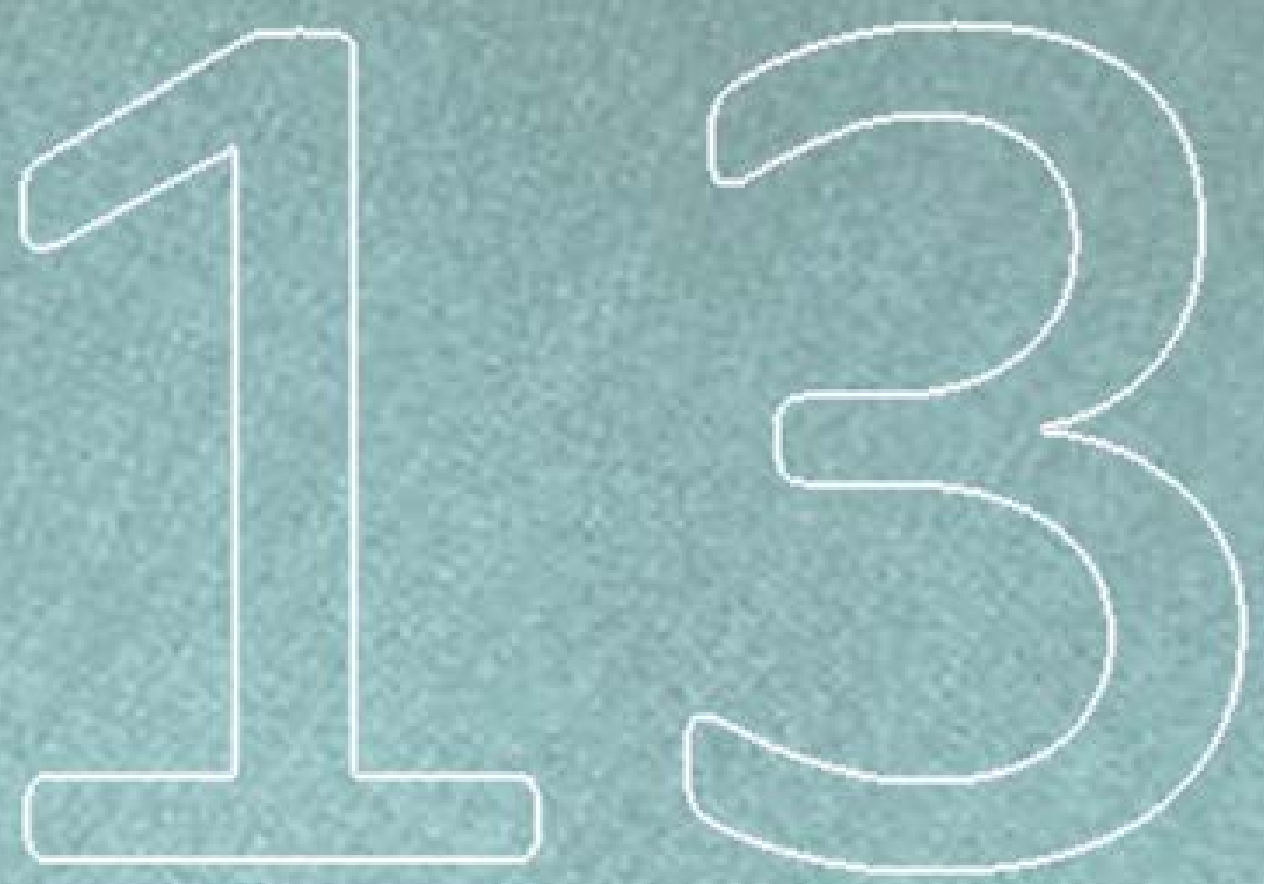

Curriculum Vitae 

Harsha Rao was born on 13th September 1976 at Shimoga, a town in South India. He completed his pre-University from DVS Junior College in Shimoga in 1994. He completed his basic medical education (in 1999) and Post-Graduation in Ophthalmology (in 2005) from Bangalore Medical College, Bangalore. He subsequently completed his long term fellowship in Glaucoma at L V Prasad Eye Institute, Hyderabad followed by another long term fellowship in Glaucoma at the University of California, San Diego. He is currently working as a consultant in the Department of Glaucoma at Narayana Nethralaya, Bengaluru. He worked as a Glaucoma consultant at L V Prasad Eye Institute, Hyderabad from 2007 to 2015 before joining Narayana Nethralaya. He had also held the positions of Adjunct Assistant Professor of Ophthalmology at the School of Medicine and Dentistry, University of Rochester Medical Center, Rochester, New York between October 2010 and April 2015, and Clinical Instructor at the School of Medicine, Case Western Reserve University, Cleveland, Ohio between July 2011 and April 2015.

He has published over 100 papers in peer-reviewed scientific journals and has been an invited speaker at multiple national and international conferences. He is on the editorial board of multiple journals and is a reviewer for most of the high impact journals in Ophthalmology. He is a member of the Associate Advisory Board of World Glaucoma Association. 